Hendrik Angelkort

\title{
Integration des
}

Rechnungswesens als

Erfolgsfaktor für die

Controllerarbeit

Eine empirische Untersuchung deutscher Großunternehmen 
Hendrik Angelkort

\section{Integration des Rechnungswesens als Erfolgsfaktor für die Controllerarbeit}

Die Integration des internen und externen Rechnungswesens ist ein Praxisphänomen, das im Laufe der letzten 15 Jahre - auch innerhalb der Wissenschaft - zunehmend an Bedeutung gewonnen hat. Um den bislang dennoch eingeschränkten empirischen Erkenntnisstand innerhalb dieses Themenfelds zu erweitern, werden die Ergebnisse einer unter den 1.500 umsatzstärksten Unternehmen Deutschlands durchgeführten Erhebung vorgestellt. Auf Basis einer umfassenden Ermittlung des Status quo der Integration des Rechnungswesens wird der Einfluss dieser Integration auf die rollenbezogene Ausgestaltung und den Erfolg der Controllerarbeit untersucht. Erstmals wird im Zuge der Analyse die Funktion von Finanzinformationen des Rechnungswesens als Sprache bzw. Kommunikationsmedium explizit berücksichtigt. Als Kernergebnis wird aufgezeigt, dass die Integration des Rechnungswesens die Entwicklung des Controllers zum Business Partner fördert und den Controllingerfolg auch aus Sicht des Managements steigert.

Hendrik Angelkort studierte Betriebswirtschaftslehre an der Universität Gießen. Während seiner anschließenden Doktorandenzeit war er Wissenschaftlicher Mitarbeiter an der dortigen Professur für Controlling und integrierte Rechnungslegung. Derzeit ist er im Group Financial Controlling eines internationalen Konzerns tätig. 
Integration des Rechnungswesens als Erfolgsfaktor für die Controllerarbeit 


\section{Controlling \& Business Accounting}

Herausgegeben von Barbara E. Weißenberger

Band 3

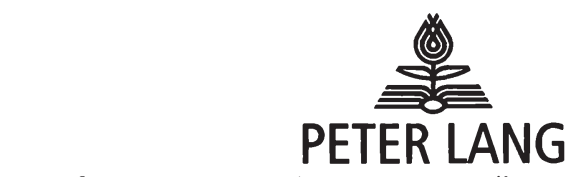

Frankfurt am Main · Berlin · Bern · Bruxelles · New York · Oxford · Wien 


\section{Hendrik Angelkort}

\section{Integration des Rechnungswesens als Erfolgsfaktor für die Controllerarbeit}

Eine empirische Untersuchung deutscher Großunternehmen

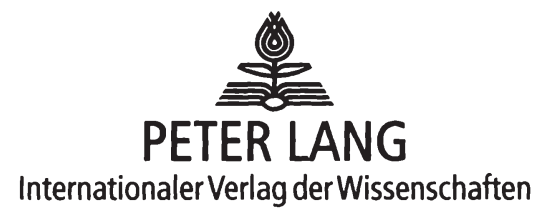




\title{
Bibliografische Information der Deutschen Nationalbibliothek
} Die Deutsche Nationalbibliothek verzeichnet diese Publikation in der Deutschen Nationalbibliografie; detaillierte bibliografische Daten sind im Internet über http://dnb.d-nb.de abrufbar.

Open Access: The online version of this publication is published on www.peterlang.com and www.econstor.eu under the international Creative Commons License CC-BY 4.0. Learn more on how you can use and share this work: http://creativecommons.org/licenses/ by/4.0.

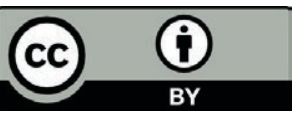

This book is available Open Access thanks to the kind support of ZBW - Leibniz-Informationszentrum Wirtschaft.

Zugl.: Gießen, Univ., Diss., 2010

\author{
Umschlaggestaltung: \\ Olaf Glöckler, Atelier Platen, Friedberg \\ Gedruckt auf alterungsbeständigem, \\ säurefreiem Papier. \\ D 26 \\ ISSN $1867-304 \mathrm{X}$ \\ ISBN 978-3-631-60379-6 \\ ISBN 978-3-631-75143-5 (eBook) \\ (C) Peter Lang $\mathrm{GmbH}$ \\ Internationaler Verlag der Wissenschaften \\ Frankfurt am Main 2010 \\ Alle Rechte vorbehalten.
}

Das Werk einschließlich aller seiner Teile ist urheberrechtlich geschützt. Jede Verwertung außerhalb der engen Grenzen des

Urheberrechtsgesetzes ist ohne Zustimmung des Verlages unzulässig und strafbar. Das gilt insbesondere für Vervielfältigungen, Übersetzungen, Mikroverfilmungen und die Einspeicherung und Verarbeitung in elektronischen Systemen. 


\section{Geleitwort}

„Different costs for different purposes“ versus „one version of the truth" - mit dieser Frage beschäftigt sich die deutsche Kostenrechnung in Theorie und Praxis seit 1994 wieder intensiv, als nämlich Siemens in dem vielzitierten Beitrag von Hasso Ziegler in der zfbf ostentativ die Abkehr von einer eigenständigen kalkulatorischen Wirtschaftsrechnung verkündete. Das Aufsetzen der internen Bereichssteuerung sowie der Zielkostenkalkulation auf die Datenbasis der externen Finanzberichterstattung, d.h. die Integration von internem und externem Rechnungswesen, stellte einen Paradigmenwechsel dar, der auch fünfzehn Jahre später noch immer wieder kontrovers diskutiert wird.

Trotz der Vielzahl von Publikationen, die das Für und Wider eines integrierten Rechnungswesens bzw. auch die konkrete Ausgestaltung in der Unternehmenspraxis erfassen, sind zentrale Fragen bisher unbeantwortet geblieben:

- Auf welchen Hierarchieebenen bzw. in welchen Aktionsfeldern der Controllerarbeit spielt die Integration von externem und internem Rechnungswesen überhaupt eine Rolle?

- Wie wirkt sich die Integration auf die angestrebte Rolle von Controllern als „Business Partner“ des Managements aus? und insbesondere

- Wird die Controllerarbeit im Kontext eines integrierten Rechnungswesens effektiver - und zwar nicht nur aus Sicht der Controller, sondern auch des Managements?

An dieser Stelle setzt die Arbeit von Hendrik Angelkort an, der diese Fragestellung erstmals empirisch mit den derzeit wohl leistungsfähigsten Instrumenten der empirischen Sozialforschung adressiert und damit einen zentralen Beitrag zur Weiterentwicklung der Theorie eines integrierten Rechnungswesens leistet. Gleichzeitig gibt Angelkort - dies sei an dieser Stelle schon vorweggegriffen - eine klare Antwort auf die drängende Frage der Unternehmenspraxis, nicht nur ob, sondern auch wie und warum die Integration von externem und internem Rechnungswesen sinnvoll ist. 
Der Arbeit von Angelkort liegt eine Stichprobe zugrunde, die aus den 1.500 umsatzstärksten Unternehmen in Deutschland gewonnen wurde. Mittels einer standardisierten schriftlichen Erhebung wurden nicht nur Controller zu ihrer Arbeit befragt, sondern auch die Sichtweise des Managements als Adressaten der Controllerarbeit auf die bereitgestellten internen Finanzberichte untersucht.

Die Ergebnisse der empirischen Analyse zeigen zunächst, dass sich in den betrachteten Unternehmen eine Integration von externem und internem Rechnungswesen weitgehend durchgesetzt hat - und das nicht nur in internationalen bzw. börsennotierten Unternehmen, sondern auch in nicht kapitalmarktorientierten Konzernen, die derzeit noch nach HGB bilanzieren. Die Integration findet dabei vor allem auf den oberen Hierarchieebenen statt; auf nachgelagerten Steuerungsebenen werden häufig auch weiterhin kalkulatorische Steuerungsgrößen verwendet.

Angelkort zeigt, dass Unternehmen mit einem hohen Integrationsgrad des Rechnungswesens die Rolle als „Business Partner“, d.h. als betriebswirtschaftlicher Berater des Managements, deutlich besser ausfüllen können. Negativ ausgedrückt: In Unternehmen mit einem eher niedrigen Integrationsgrad dominiert die Rolle des Controllers als Methoden- und Systemdienstleister. Ein wesentlicher Treiber der Beraterrolle ist interessanterweise die Integration innerhalb der IT-Systeme, die im externen und internen Rechnungswesen eingesetzt werden. Schon an dieser Stelle werden für die Controllingpraxis wichtige Hinweise für die Ausgestaltung der Unternehmensrechnung als zentralem Instrument der Controllerarbeit gegeben.

Wegweisenden Charakter erhält die Arbeit Angelkorts durch die Beantwortung der Frage nach der Effektivität der Controllerarbeit im Zuge einer Integration des Rechnungswesens. Angelkort beschränkt sich hier nicht auf das in der Literatur übliche instrumentelle Verständnis des Rechnungswesens als Informationsgrundlage für Entscheidungsfundierung und -steuerung, sondern prüft eine erweiterte Modellierung, die die Bedeutung der Rechnungslegung als Finanzsprache, d.h. als konzeptionelles Medium zum Wissensaufbau und Kommunikation über unternehmensrelevante Sachverhalte, erfasst.

Das Kernergebnis der empirischen Überprüfung besagt hier zunächst, dass die Integration des Rechnungswesens - entgegen bestehenden Vermutungen - per se keinen direkten, d.h. instrumentellen Effekt auf die Effektivität der Controllerarbeit aus Managersicht besitzt. Ein Einfluss entsteht erst dann, wenn die Integration des 
Rechnungswesens durch die Controller auch auf konzeptioneller Ebene umgesetzt wird, d.h. eine einheitliche Finanzsprache begründet, die ein konsistentes Geschäftsverständnis und Unternehmensabbild sowohl für externe als auch für interne Adressaten sicherstellt. Dieses Ergebnis wird bestätigt, wenn man zu allen Sachverhalten lediglich Controller befragt - allerdings zeigt sich hier, dass die Controller die Bedeutung einer einheitlichen Finanzsprache für die Effektivität ihrer Arbeit deutlich unterschätzen.

Methodische Grundlage dieser sowohl für die betriebswirtschaftliche Theorie als auch für die Unternehmenspraxis äußerst bedeutsamen Ergebnisse ist eine Kovarianzstrukturanalyse, die sowohl die Einschätzungen von Controllern als auch von Managern erfasst. Durch die aufwändige Vorgehensweise werden wichtige konzeptionelle Kritikpunkte an empirischen Untersuchungen wie Common Method Bias oder Key Informant Bias, die zu Verzerrungen in der Datenauswertung führen können, vermieden. Die Ergebnisse zeichnen sich so durch eine hohe Reliabilität und Validität aus.

Wissenschaftstheoretisch steht die Dissertationsschrift Angelkorts in der Tradition der Arbeiten von Eugen Schmalenbach - nicht nur deshalb, weil sie die Frage nach dem ,richtigen“ Betriebsgewinn wieder aufgreift. Vielmehr stellt sie sich auch der bereits 1911 von Schmalenbach geäußerten Forderung, „Privatwirtschaftslehre als Kunstlehre" zu verstehen, d.h. betriebswirtschaftliche Theorieentwicklung und empirische Analyse werden dergestalt verbunden, dass belastbare Handlungsimplikationen für die Nutzung eines integrierten Rechnungswesens innerhalb der Controllerarbeit abgeleitet werden können.

Die Arbeit von Hendrik Angelkort ist deshalb für einen breiten Leserkreis sowohl in Unternehmen als auch in der betriebswirtschaftlichen Forschung von höchster Relevanz. Eine klare und präzise Sprache verbunden mit der Fokussierung auf die wesentlichen Argumente sowie eine didaktisch äußerst geschickte Führung nehmen den Leser mit auf eine spannende Reise in das Gebiet der Unternehmensrechnung. Ich wünsche dieser mit der Höchstnote ausgezeichneten Dissertationsschrift die positive Aufnahme in der Community, die sie verdient. 
Hendrik Angelkort - 978-3-631-75143-5

Downloaded from PubFactory at 01/11/2019 07:47:42AM

via free access 


\section{Vorwort}

Die vorliegende Dissertation entstand während meiner Zeit als wissenschaftlicher Mitarbeiter an der Professur für Controlling und integrierte Rechnungslegung an der Justus-Liebig-Universität Gießen. Ohne die Unterstützung zahlreicher Personen wäre die Fertigstellung dieser Arbeit nicht möglich gewesen. Ihnen möchte ich an dieser Stelle meinen Dank aussprechen.

Ganz besonders herzlich bedanke ich mich bei meiner akademischen Lehrerin, Frau Prof. Dr. Barbara E. Weißenberger, für die hervorragende Betreuung meines Promotionsprojekts. Ihr hohes persönliches Engagement in Forschung und Lehre, die von ihr etablierte Kultur des Forderns und Förderns sowie letztendlich ihre Fairness und menschliche Großzügigkeit haben meine Zeit an der Professur in entscheidender Weise positiv geprägt. Durch ihre stete Gesprächsbereitschaft sowie die Ermöglichung der Teilnahme an zahlreichen (inter)nationalen Konferenzen hat Frau Prof. Weißenberger ganz wesentlich zum Gelingen der vorliegenden Dissertation sowie meiner akademischen Ausbildung beigetragen.

Herrn Prof. Dr. Rüdiger Kabst danke ich für die Übernahme des Korreferats sowie für die Möglichkeit der Teilnahme am Doktorandenseminar seiner Professur.

Frau Dr. Rita Niedermayr-Kruse vom Österreichischen Controller-Institut danke ich für die finanzielle und operative Unterstützung der Studie 'Controller Excellence unter IFRS in Österreich'. Die im Zuge dieses Projekts gewonnenen Erfahrungswerte waren für die Realisierung der vorliegenden empirischen Untersuchung sehr wertvoll. Tatkräftig unterstützt wurde ich bei dieser wiederum von Julia Blöcher, Nina Franzen, Brigitte Richter, Gero Holthoff und Stefan Rausch. Ihnen allen gilt ebenfalls mein Dank.

Darüber hinaus bedanke ich mich bei Dr. Christoph Gehrig, Prof. Dr. Joachim Sandt, Prof. em. Dr. Peter Schmidt, Dr. Holger Steinmetz, Sebastian Wolf sowie allen Teilnehmern des Doktorandenkolloquiums der Professur für die konstruktiven Diskussionen und wertvollen Hinweise hinsichtlich der Konzeption, Durchführung und Auswertung der vorliegenden empirischen Studie.

Danken möchte ich zudem meinen ehemaligen Kollegen vom Team BWL IV, insbesondere Anneke Behrendt, Dr. Marcus Blome, Nina Franzen, Dr. Christoph 
Gehrig, Gero Holthoff, Benjamin Löhr und Sebastian Wolf. Mich in einem Umfeld bewegen zu dürfen, in dem die Grenzen zwischen fachlicher Zusammenarbeit und Freundschaft fließend verlaufen, habe ich stets als Privileg angesehen.

Der größte Dank gebührt den Personen aus meinem persönlichen Umfeld, die mir den notwendigen Rückhalt für ein Projekt dieser Größenordnung gegeben haben. Bei meinen Eltern bedanke ich mich dafür, dass sie den von mir eingeschlagenen Ausbildungsweg ermöglicht und mir dabei jegliche nur denkbare Unterstützung gewährt haben. Meiner Frau Suset danke ich für ihre Liebe und Geduld. Sie hat mich stets in der Verfolgung meiner Ziele bestärkt. Durch den ihr eigenen Optimismus sowie ihre Lebensfreude hat sie an jedem einzelnen Tag - unabhängig von aufziehenden Wolken am Dissertationshimmel - für Sonnenschein gesorgt.

Frankfurt am Main, im April 2010

Hendrik Angelkort 


\section{Inhaltsverzeichnis}

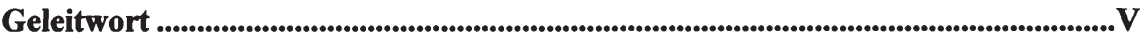

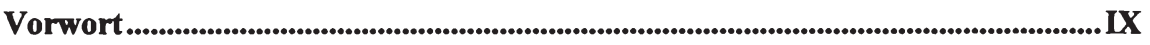

Abbildungsverzeichnis.........................................................................................................XV

Tabellenverzeichnis........................................................................................................... XVII

Abkürzungsverzeichnis ............................................................................................................... XIX

Symbolverzeichnis........................................................................................................................XXIII

A Einleitung ......................................................................................................................................... 1

1 Problemstellung und Zielsetzung .................................................................. 1

2 Wissenschaftstheoretische Einordnung und Gang der Arbeit .......................... 9

B Rechnungswesen als Instrument für die Controllerarbeit ..................................... 13

1 Controlling als spezielle Führungsfunktion..................................................... 13

1.1 Controllingverständnis .................................................................... 13

1.2 House of Controlling als konzeptioneller Bezugsrahmen der Controllerarbeit .................................................................................... 14

2 Grundlagen des betrieblichen Rechnungswesens im deutschsprachigen

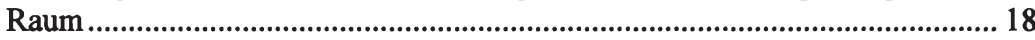

2.1 Begriff und Aufgabe des Rechnungswesens........................................... 18

2.2 Traditionelle Teilbereiche des Rechnungswesens .................................... 20

3 Ansatzpunkte für eine Integration von internem und externem

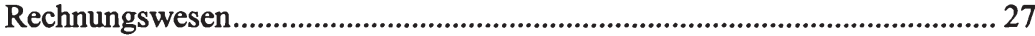

3.1 Konzept eines integrierten Rechnungswesens ....................................... 27

3.2 Muster einer partiellen Integration des Rechnungswesens..................... 31

4 Stand der empirischen Forschung zur Integration des Rechnungswesens ...... 34

4.1 Überblick über bisherige Studien........................................................... 34

4.2 Wesentliche Ergebnisse ............................................................................. 39

C Grundlagen der empirischen Untersuchung ........................................................ 49

1 Konzeption und Ablauf der empirischen Untersuchung .................................. 49

1.1 Untersuchungsziel und Forschungsdesign .............................................. 49

1.2 Durchfuihrung der Datenerhebung ........................................................ 50 


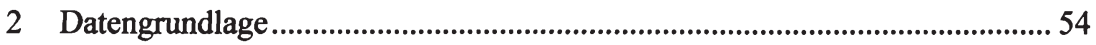

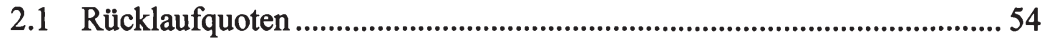

2.2 Stichprobenmerkmale ............................................................................... 56

3 Methodische Grundlagen der Untersuchung .................................................... 59

3.1 Skalenniveau der Untersuchungsdaten .................................................... 59

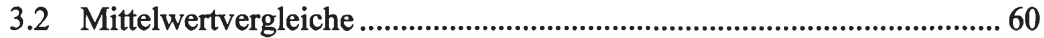

3.3 Kreuztabellen und Chi-Quadrat-Unabhängigkeitstest ............................. 63

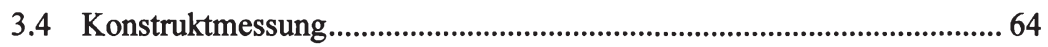

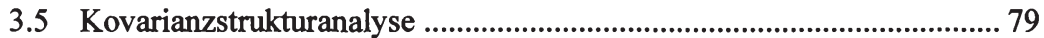

D Status quo der Integration des Rechnungswesens in deutschen GroBunternehmen ......................................................................................................................... 89

1 Integration des internen und externen Rechnungswesens innerhalb der Aktionsfelder der Controllerarbeit................................................................89

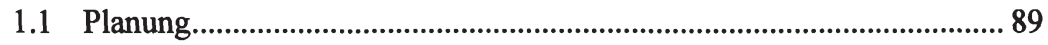

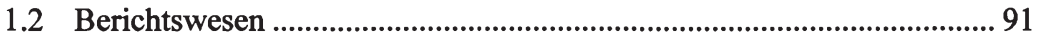

1.3 Performance-Messung ............................................................................... 94

1.4 Gestaltung der controllingrelevanten IT-Systeme ................................... 95

1.5 Institutionalisierung der Controllerarbeit............................................... 97

2 Bildung von Integrationsindizes als $\mathrm{Ma} ß$ der Integrationsintensität von internem und externem Rechnungswesen ........................................................ 98

2.1 Vorgehensweise bei der Indexbildung .................................................... 98

2.2 Integrationsintensität der Aktionsfelder der Controllerarbeit ................. 100

2.3 Ableitung von kontextspezifischen Integrationsprofilen........................ 101

3 Stand der Umsetzung des Musters einer partiellen Integration des

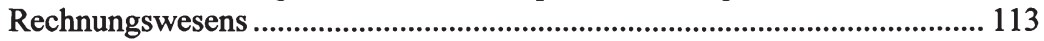

3.1 Identifizierung und Quantifizierung des Musters einer partiellen Integration des Rechnungswesens

3.2 Einfluss von Kontextfaktoren auf die Umsetzung unterschiedlicher Integrationsmuster............................................................................... 118

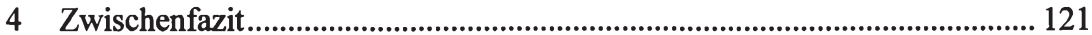


E Einfluss der Integration des Rechnungswesens auf die Controllerarbeit..... 123

1 Rollenbezogene Ausgestaltung der Controllerarbeit im Kontext der Integration des Rechnungswesens ................................................................ 123

1.1 Ausübung der Controllerrollen ............................................................. 123

1.2 Einfluss der Integrationsintensität des Rechnungswesens auf die Ausübung der Controllerrollen

2 Einfluss der Integration des Rechnungswesens auf den Erfolg der Controllerarbeit.

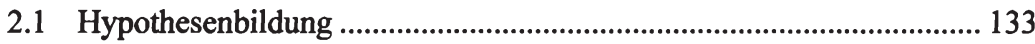

2.2 Operationalisierung der latenten Variablen ......................................... 137

2.3 Gütebeurteilung der Konstruktmessung ........................................... 141

2.4 Überprüfung der Hypothesen des Kovarianzstrukturmodells ............... 148

2.5 Mehrgruppenanalyse zur Identifizierung eines moderierenden Effektes der Gruppenzugehörigkeit der Antwortenden auf die Dependenzbeziehungen

3 Implikationen der Untersuchungsergebnisse zum Einfluss der Integration des Rechnungswesens auf die Controllerarbeit

F Schlussbetrachtung .................................................................................................................. 163

1 Zusammenfassung der zentralen Ergebnisse ............................................... 163

2 Kritische Würdigung und Ausblick auf die weitere Forschung ..................... 167

Literaturverzeichnis .............................................................................................. 173 
Hendrik Angelkort - 978-3-631-75143-5

Downloaded from PubFactory at 01/11/2019 07:47:42AM

via free access 


\section{Abbildungsverzeichnis}

Abbildung 1: Deutschsprachige Publikationen zur Integration des

Rechnungswesens im Zeitraum von 1994 bis 2007 .............................. 3

Abbildung 2: Gang der Arbeit ................................................................................... 10

Abbildung 3: House of Controlling .......................................................................... 15

Abbildung 4: Abgrenzung von Aufwendungen und Kosten ..................................... 22

Abbildung 5: Gegenüberstellung von separiertem und integriertem Rechnungs-



Abbildung 6: Partielle Integration des Rechnungswesens.......................................... 32

Abbildung 7: Verknüpfung der Forschungsfragen mit dem House of Controlling.. 49

Abbildung 8: Stichprobenabgrenzung ........................................................................ 55

Abbildung 9: Branchenzusammensetzung der Stichproben ........................................ 56

Abbildung 10: Stichprobenmerkmale 'Börsennotierung' und 'Führender Rechnungslegungsstandard' .......................................................................... 58

Abbildung 11: Beispielhaftes Kovarianzstrukturmodell ............................................... 81

Abbildung 12: Gesamtintegrationsindex und Integrationssubindizes der Aktionsfelder der Controllerarbeit .............................................................. 100

Abbildung 13: Integrationsprofil in Abhängigkeit der Börsennotierung .................. 102

Abbildung 14: Integrationsprofil in Abhängigkeit der Unternehmensgröße............. 104

Abbildung 15: Integrationsprofil in Abhängigkeit des führenden Rechnungslegungsstandards

Abbildung 16: Integrationsprofil in Abhängigkeit der Hierarchiestufe innerhalb eines Konzernverbundes.............................................................. 108

Abbildung 17: Integrationsprofil in Abhängigkeit der Volatilität des operativen Ergebnisses

Abbildung 18: Integrationsprofil in Abhängigkeit der Änderungsdynamik des Unternehmensumfelds

Abbildung 19: Hinweise auf eine partielle Integration des Rechnungswesens innerhalb der operativen Ergebnisplanung

Abbildung 20: Identifizierte Integrationsmuster innerhalb der operativen Ergebnisplanung

Abbildung 21: Bisherige und angestrebte Ausübung der Controllerrollen 
Abbildung 22: Ausübung der Controllerrollen in Abhängigkeit der Gesamtintegrationsintensität des Rechnungswesens

Abbildung 23: Ausübung der Controllerrollen in Abhängigkeit der Integrationsintensität des Rechnungswesens innerhalb der originären Aktionsfelder der Controllerarbeit

Abbildung 24: Ausübung der Controllerrollen in Abhängigkeit der Integrationsintensität des Rechnungswesens innerhalb der derivativen Aktionsfelder der Controllerarbeit.

Abbildung 25: Dyadisches Untersuchungsmodell

Abbildung 26: Messmodell der Untersuchung hinsichtlich der endogenen latenten Variablen in Matrixnotation. 144

Abbildung 27: Strukturmodell in Matrixnotation 148

Abbildung 28: Ergebnisse der Parameterschätzung 149 


\section{Tabellenverzeichnis}

Tabelle 1: Gegenüberstellung von externem und internem Rechnungswesen ........ 20

Tabelle 2: Empirische Studien zur Integration des Rechnungswesens...................... 35

Tabelle 3: Fragebogeninhalte............................................................................ 51

Tabelle 4: Größenkriterien der Stichproben.........................................................57

Tabelle 5: Entscheidungsfragen zur Bestimmung der geeigneten Konstrukt-

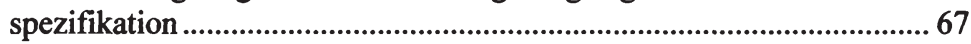

Tabelle 6: Gütekriterien zur Beurteilung der reflektiven Messmodelle ................... 78

Tabelle 7: Integration des Rechnungswesens im Bereich Planung ........................... 90

Tabelle 8: Integration des Rechnungswesens im Bereich Berichtswesen ................ 93

Tabelle 9: Integration des Rechnungswesens im Bereich Performance-Messung .. 95

Tabelle 10: Integration des Rechnungswesens im Bereich Gestaltung der controllingrelevanten IT-Systeme.............................................................96

Tabelle 11: Integration des Rechnungswesens im Bereich Institutionalisierung

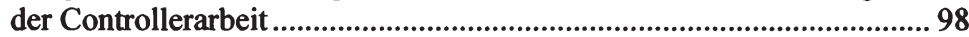

Tabelle 12: Ermittlung von Integrationsmustern innerhalb der operativen Ergebnisplanung..................................................................................... 116

Tabelle 13: Kreuztabelle Integrationsmuster und Börsennotierung ........................... 118

Tabelle 14: Chi-Quadrat-Test für Kreuztabelle Integrationsmuster und Börsennotierung

Tabelle 15: Kreuztabelle Integrationsmuster und Leading GAAP 120

Tabelle 16: Chi-Quadrat-Test für Kreuztabelle Integrationsmuster und Leading GAAP

Tabelle 17: Operationalisierung des Faktors 'Ergebnisqualität des Controllings' ... 140

Tabelle 18: Operationalisierung des Faktors 'Einheitlichkeit der Finanzsprache' ... 140

Tabelle 19: Operationalisierung des Faktors 'Einfluss des Controllings auf Managemententscheidungen'

Tabelle 20: Informationen zum Faktor 'Ergebnisqualität des Controllings' ............. 145

Tabelle 21: Informationen zum Faktor 'Einheitlichkeit der Finanzsprache' ............. 146

Tabelle 22: Informationen zum Faktor 'Einfluss des Controllings auf Managemententscheidungen' 
Tabelle 23: Ergebnisse der Überprüfung der Diskriminanzvalidität anhand des Fornell/Larcker-Kriteriums

Tabelle 24: Anpassungsgüte des Kovarianzstrukturmodells ...................................... 148

Tabelle 25: Ergebnisse der Effektzerlegung ............................................................... 151

Tabelle 26: Detailanalyse der aktionsfeldbezogenen Submodelle.............................. 153

Tabelle 27: Ergebnisse der kovarianzstrukturanalytischen Mehrgruppenanalyse zur Identifizierung eines moderierenden Effektes 


\section{Abkürzungsverzeichnis}

AG Aktiengesellschaft

AGFI Adjusted Goodness of Fit Index

AT Arbeitstage

Aufl. Auflage

BilMoG Bilanzrechtsmodernisierungsgesetz

bzw. beziehungsweise

C.R. Critical Ratio

ca. circa

CFI Comparative Fit Index

CFO Chief Financial Officer

d. Verf. der Verfasser

d.h. das heißt

DAX Deutscher Aktienindex

DEV durchschnittlich erfasste Varianz

DRS Deutsche(r) Rechnungslegungs Standard(s)

e.g. exempli gratia

EBIT Earnings before Interest and Tax

EBITDA Earnings before Interest, Tax, Depreciation, and Amortization

ERP Enterprise Resource Planning

et al. et alii

etc. et cetera

EU Europäische Union

EUR Euro

EVA Economic Value Added 
F\&E $\quad$ Forschung \& Entwicklung

f. folgende

ff. fortfolgende

GAAP Generally Accepted Accounting Principles

GFI Goodness of Fit Index

ggf. gegebenenfalls

$\mathrm{GuV} \quad$ Gewinn- und Verlustrechnung

HGB Handelsgesetzbuch

Hrsg. Herausgeber

i.d.R. in der Regel

i.e. id est

i.e.S. im engeren Sinne

IAS International Accounting Standard(s)

IASB International Accounting Standards Board

ICV Internationaler Controller Verein

IFRS International Financial Reporting Standard(s)

IGC International Group of Controlling

IT Information Technology

Jg. Jahrgang

KapAEG Kapitalaufnahmeerleichterungsgesetz

KMU kleine und mittlere Unternehmen

KPI(s) Key Performance Indicator(s)

LISREL Linear Structural Relations

LSP Leitsätze für die Preisbildung aufgrund von Selbstkosten

m.w.N. mit weiteren Nachweisen

MA Management Accounting 
MDAX Midcap-DAX

MIMIC Multiple Indicators, Multiple Causes

Mio. Millionen

ML Maximum Likelihood

NNFI Non-Normed Fit Index

Nr. Nummer

PLS Partial Least Squares

RMR Root Mean Square Residual

RMSEA Root Mean Square Error of Approximation

Rz. Randziffer(n)

S. $\quad$ Seite(n)

SDAX Smallcap-DAX

SEM Structural Equation Modeling

SMM Shared Mental Model(s)

SRMR Standardized Root Mean Square Residual

TLI Tucker Lewis Index

US United States

USA United States of America

Vgl. Vergleiche

Vol. Volume

vs. versus

z.B. zum Beispiel 
Hendrik Angelkort - 978-3-631-75143-5

Downloaded from PubFactory at 01/11/2019 07:47:42AM

via free access 


\section{Symbolverzeichnis}

Das Symbolverzeichnis enthält die Symbole, die mehrfach in Textpassagen, Abbildungen oder Tabellen verwendet werden. Wird ein Symbol lediglich an einer Stelle verwendet, findet sich die entsprechende Erläuterung aus Gründen der Übersichtlichkeit direkt vor Ort.

$\begin{array}{ll}\chi^{2} & \text { Chi-Quadrat } \\ \text { df } & \text { Freiheitsgrade (degrees of freedom) } \\ \text { H } & \text { Hypothese } \\ \text { MW } & \text { Mittelwert } \\ \text { N } & \text { Stichprobengröße } \\ \text { p } & \text { Signifikanzniveau } \\ \text { SA } & \text { Standardabweichung }\end{array}$


Hendrik Angelkort - 978-3-631-75143-5

Downloaded from PubFactory at 01/11/2019 07:47:42AM

via free access 
A Einleitung

„Kein Aufsichtsrat ist besonders glücklich, wenn ihm in der Aufsichtsratssitzung vier bis fünf verschiedene Ergebnisse präsentiert werden: ein handelsrechtliches, ein steuerrechtliches, ein betriebswirtschaftliches, ein Ergebnis vor Umrechnungen und nach Umrechnungen etc., und auf die simple Frage, was haben wir denn wirklich verdient, keine klare Antwort kommt. "

Havermann (2000), S. 123

\section{$1 \quad$ Problemstellung und Zielsetzung}

Die von Havermann (2000) treffend formulierte Aussage verdeutlicht das Kernproblem der auf Schmalenbach ${ }^{1}$ zurückgehenden Tradition einer Separation von internem und externem Rechnungswesen im deutschsprachigen Raum. ${ }^{2}$ So ist es zwar möglich, die in einem Zweikreissystem auftretenden Divergenzen zwischen dem internen Ergebnis der kalkulatorischen Managementerfolgsrechnung und dem externen pagatorischen Ergebnis der Gewinn- und Verlustrechnung $(\mathrm{GuV})$ konzeptionell zu erläutern. ${ }^{3}$ Häufig können diese aber nicht mehr inhaltlich belegt werden und führen aufgrund der hieraus resultierenden Widersprüchlichkeit und mangelnden Kommunizierbarkeit zu einem Glaubwürdigkeitsproblem der seitens des Controllings bereitgestellten Finanzinformationen - insbesondere für den Fall, dass das intern ermittelte Ergebnis deutlich höher oder niedriger ausfällt als das externe. ${ }^{4}$

Um diese „Doppelzüngigkeit ${ }^{\star 5}$ der Ergebnisrechnung zu durchbrechen, entschied sich die Siemens AG mit Beginn des Geschäftsjahres 1992/93 ihre bis dato eigenständige interne Erfolgsrechnung aufzugeben. ${ }^{6}$ Stattdessen wurde fortan als neue zentrale

1 Für einen Überblick über die Denkansätze Schmalenbachs zur Gestaltung der Erfolgsrechnung vgl. Weißenberger (2003), S. 177ff.

2 Für eine Abgrenzung des Begriffs des Rechnungswesens vgl. die Ausfuhrungen in Abschnitt B2.1 der vorliegenden Arbeit.

3 Vgl. auch im Folgenden Bruns (1999), S. 593 sowie Weißenberger/Angelkort (2007a), S. 424.

4 Vgl. Währisch (1998), S. 119 sowie Wagenhofer (2006), S. 13.

5 Weißenberger (2007a), S. 192.

6 Vgl. auch im Folgenden Ziegler (1994), S. 177ff. 
Steuerungsgröße auf ein operatives Ergebnis zurückgegriffen, welches sich in einfacher Weise aus dem Ergebnis der gewöhnlichen Geschäftstätigkeit der - erstmalig nach dem Umsatzkostenverfahren aufgestellten - GuV herleiten ließ. ${ }^{7}$ Mit Ausnahme der berücksichtigten Verzinsung des betriebsnotwendigen Vermögens bedeutete diese Umstellung eine Abkehr von der Verwendung kalkulatorischer Elemente innerhalb der internen Erfolgsrechnung. Ausschlaggebend für die Neuorientierung des internen Rechnungswesens war hierbei der Wunsch nach einer einheitlichen und transparenten Finanzsprache. Insbesondere hielt man die Zweiteilung des Rechnungswesens auf internationaler Ebene für nicht vermittelbar. ${ }^{8}$

Der seitens des Siemens-Konzerns vollzogene Paradigmenwechsel löste in Theorie und Unternehmenspraxis eine bis heute andauernde Diskussion über die Integration ${ }^{9}$ des internen und externen Rechnungswesens aus. ${ }^{10}$ Einen Beleg hierfür bilden die in Abbildung 1 dargestellten Ergebnisse einer Analyse sämtlicher deutschsprachiger Publikationen zu diesem Themenbereich für den Zeitraum von 1994 bis $2007 .^{11}$ So zeigt sich, dass sich die Anzahl der Veröffentlichungen - und somit zugleich die der Thematik entgegengebrachte Aufmerksamkeit - in zwei Wellen entwickelt hat. Ende der 1990er-Jahre intensivierte sich erstmalig die diesbezügliche wissenschaftliche

7 Das operative Ergebnis unterschied sich lediglich durch drei Überleitungspositionen vom GuVErgebnis. So enthielt es kein Beteiligungs-, Zins- und übriges Finanzergebnis und wurde zudem um alle aus dem Imparitätsprinzip resultierenden Aufwendungen und Erträge bereinigt. Darüber hinaus wurde das operative Ergebnis um kalkulatorische Zinsen auf das betriebsnotwendige Vermögen korrigiert. Vgl. Ziegler (1994), S. 178f.

Vgl. Ziegler (1994), S. 177 sowie Weißenberger (2007b), S. 13. Demnach erfolgte die Integration des Rechnungswesens nicht aus dem Druck internationaler Rechnungslegungsstandards heraus, da die Siemens AG zu diesem Zeitpunkt ihre Finanzberichterstattung noch nach den Regelungen des HGB aufstellte, vgl. Weißenberger (2007b), S. 13 sowie Wagenhofer (2008), S. 161.

In der Literatur werden synonym hierfür häufig auch die Begriffe 'Harmonisierung', 'Konvergenz' oder 'Vereinheitlichung' verwendet, vgl. Küting/Lorson (1999), S. 47, Schaier (2007), S. 108 sowie Simons/Weißenberger (2009a), S. 391.

10 Die erste Reaktion auf den Beitrag von Ziegler (1994) stellte im Rahmen dieser Diskussion die direkte Antwort von Pfaff (1994) dar. Ein aktuelles Beispiel für das nachhaltige Interesse an der Thematik der Integration des Rechnungswesens liefern die diesbezüglich von den Leitern Konzerncontrolling der DAX 30-Unternehmen gesammelten Kommentare bei Weber (2008), S. 237ff. In der von Janusiak/Wittkowski (2008) durchgeführten Analyse wurden Beiträge aus den führenden deutschsprachigen Fachzeitschriften mit controlling- bzw. rechnungslegungsspezifischer Ausrichtung, Beiträge aus Sammelbänden, Monographien sowie Forschungspapiere berücksichtigt, die sich mit der Integration des internen und externen Rechnungswesens auseinandersetzen. Der relevante Untersuchungszeitraum wurde dabei anhand des Erscheinungsjahrs von Zieglers Beitrag abgegrenzt, da dieser - wie bereits beschrieben - den Ausgangspunkt der Integrationsdiskussion bildet. Vgl. Janusiak/Wittkowski (2008), S. 10-12. 
Diskussion, was auf die steigende Kapitalmarktorientierung der Unternehmen und die damit einhergehende zunehmende Verbreitung wertorientierter Steuerungskonzepte sowie auf das Voranschreiten der Internationalisierung der Rechnungslegung zurückgeführt werden kann. ${ }^{12}$

Insgesamt 339 deutschsprachige Publikationen zur Integration des Rechnungswesens

Davon 104 empirisch ausgerichtet

Gesteigerte Bedeutung des Themas aufgrund verpflichtender IFRSUmstellung für kapitalmarktorientierte Konzerne ab 2005/2007

Hohe Bedeutung der Integration aufgrund steigender Kapitalmarktorientierung und Internationalisierung der Rechnungslegung

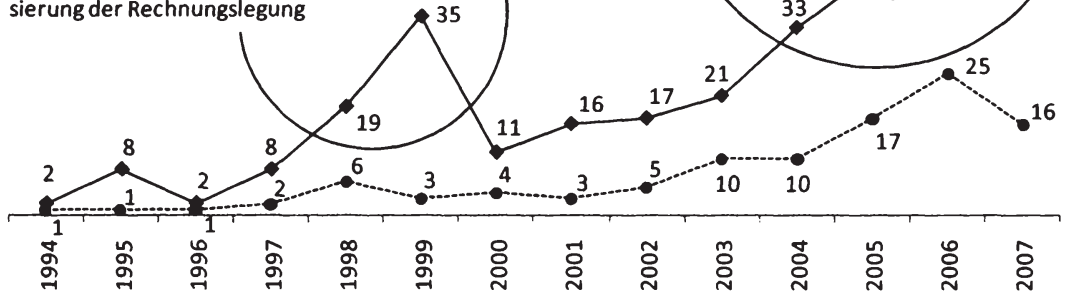

Abbildung 1: Deutschsprachige Publikationen zur Integration des Rechnungswesens im Zeitraum von 1994 bis 2007

Die zweite Wellenbewegung innerhalb der Intensität der Integrationsdiskussion erfolgte zwischen 2004 und 2007. Ausschlaggebend hierfür war die gesetzliche Ver-

Vgl. Küting/Lorson (1998), S. 490f., Bruns (1999), S. 593, Heyd (2001), S. 206, Kümpel (2002), S. 343f., Weißenberger/Angelkort (2007a), S. 425f., Günther/Zurwehme (2008), S. 101 sowie Wagenhofer (2008), S. 161. Von besonderer Bedeutung für diese Entwicklung war das Anfang 1998 erlassene Kapitalaufnahmeerleichterungsgesetz (KapAEG), durch das börsennotierte Mutterunternehmen gemäß §292a HGB von der Verpflichtung zur Aufstellung eines Konzernabschlusses nach HGB-Vorschriften befreit wurden, wenn stattdessen ein Konzernabschluss nach international anerkannten Rechnungslegungsstandards - etwa IFRS oder US-GAAP - erstellt wurde. Vgl. Küting/Lorson (1999), S. 50, Hoke (2001), S. 69-71 sowie Weber/Weißenberger (2001), S. 542. Vor diesem Hintergrund wird die Internationalisierung der Rechnungslegung auch als Katalysator für die Integration des Rechnungswesens bezeichnet, vgl. Hoke (2001), S. 62. 
pflichtung für kapitalmarktorientierte Unternehmen mit Sitz in der Europäischen Union, ihren Konzernabschluss ab 2005 oder spätestens 2007 nach Maßgabe der International Financial Reporting Standards (IFRS) aufzustellen. ${ }^{13}$ So bilden die IFRS einen wichtigen Kontextfaktor bzw. Treiber der Integration des Rechnungswesens, da sie aufgrund ihrer ökonomisch geprägten Perspektive eine im Vergleich zum Handelsgesetzbuch (HGB) deutlich geeignetere Grundlage für die interne Berichterstattung bilden. ${ }^{14}$ Im Zuge der durch die EU-Verordnung weiter gestiegenen Verbreitung der IFRS erhöhte sich daher abermals das Interesse an der Integration des internen und externen Rechnungswesens, was sich in einer zuvor nicht erreichten Anzahl an Publikationen zu diesem Themenfeld innerhalb des betrachteten Zeitraums der zweiten Wellenbewegung widerspiegelt.

Trotz einer breiten empirisch ausgerichteten Literaturbasis zur Integration des Rechnungswesens - hierzu zählen 104 der insgesamt 339 Veröffentlichungen - bestehen in diesem Feld bislang noch umfangreiche Forschungslücken. So befinden sich unter den besagten 104 Beiträgen 22 großzahlige Untersuchungen, ${ }^{15}$ von denen sich jedoch lediglich neun schwerpunktmäßig mit dem Thema der Integration des Rechnungswesens befassen. ${ }^{16}$ Kennzeichnend für diese Studien, die bis auf eine Ausnahme erst ab dem Jahr 2001 erschienen sind, ist wiederum die überwiegend deskriptiv-

13 Diese Regelung basiert auf der EU-Verordnung Nr. 1606/2002 zur Anwendung internationaler Rechnungslegungsstandards (IAS-Verordnung). Als kapitalmarktorientiert gelten dabei Unternehmen, deren Eigen- oder Fremdkapitalpapiere an einem regulierten Markt innerhalb der Europäischen Union notiert sind, vgl. Artikel 4 und 9 der IAS-Verordnung. Vgl. International Group of Controlling (Hrsg.)/Weißenberger (2006), S. 25ff., Weißenberger (2007c), S. 322ff., Weißenberger/Angelkort (2007a), S. 426 sowie Simons/Weißenberger (2009b), S. 446f. Die Wirkung der IFRS als Treiber der Integration des Rechnungswesens wurde auch bereits empirisch nachgewiesen. So gaben $20 \%$ der von Weißenberger/Angelkort (2007b) befragten österreichischen IFRS-Anwender an, die Integration des internen und externen Rechnungswesens im Zuge der IFRS-Umstellung durchgefüht zu haben, vgl. Weißenberger/Angelkort (2007b), S. 28.

15 Bei den übrigen 82 Publikationen handelt es sich um unternehmensbezogene Einzelberichte, Fallstudien und Interviews. Diese basieren aufgrund von Mehrfachdarstellungen einiger Unternehmen - hierzu zählen insbesondere Siemens, Daimler, Bayer, Deutsche Telekom, Deutsche Lufthansa, Henkel, Bosch und SAP - allerdings lediglich auf 35 verschiedenen Unternehmen.

16 Die zentralen Ergebnisse dieser sowie weiterer seitdem erschienener Studien werden in Abschnitt B4 der vorliegenden Arbeit vorgestellt. 
explorative Vorgehensweise. ${ }^{17}$ So erfolgt die empirische Analyse des aus der Praxis hervorgegangenen Integrationsphänomens hier weitestgehend nicht auf Basis zuvor aufgestellter Hypothesen, was auf einen noch eingeschränkten Erkenntnisstand innerhalb dieses Untersuchungsfeldes zurückgeführt werden kann. ${ }^{18}$ Dieses Muster entspricht strukturell der Einschätzung von Zimmerman (2001):

„The empirical managerial [accounting] literature focuses on describing current accounting practice. Most other accounting research areas also started descriptively, but as empirical findings accumulated, theories were developed to explain what was observed and to predict phenomena yet to be observed. [...] The literature has failed to move from describing practice to developing and testing theories, as have other accounting areas. " 19

Zwar bezieht sich diese Feststellung auf die englischsprachige Management Accounting-Literatur; sie lässt sich aber auf das Themenfeld der vorliegenden Arbeit übertragen. So kann in analoger Weise konstatiert werden, dass die Integration des Rechnungswesens im Rahmen der bisherigen - in deskriptiv-explorativer Weise durchgeführten - empirischen Studien insgesamt zwar überwiegend positiv beurteilt wird. ${ }^{20}$ Eine explanative Untersuchung, die den Einfluss der Integration auf die rollenbezoge-

17 In der empirischen Forschung kann zwischen drei idealtypischen Untersuchungstypen differenziert werden. Deskriptive Untersuchungen verfolgen das Ziel, Populationen (Grundgesamtheiten) hinsichtlich ausgewăhlter Merkmale zu beschreiben. Hingegen dienen explorative Untersuchungen der Generierung von Theorien und Hypothesen. Sie sind daher innerhalb des wissenschaftlichen Arbeitsprozesses den explanativen Untersuchungen vorgeschaltet, die wiederum auf die Überprüfung von Theorien und Hypothesen abzielen. Zwar gehen auch explorative Untersuchungen nicht vollständig theoriefrei vor, da bereits die Auswahl und Operationalisierung der Untersuchungsvariablen sowie die Selektion der Untersuchungsobjekte durch implizite und/oder explizite Vorannahmen und Theorien geleitet wird. Allerdings ist dieses theoretische Vorverstandnis noch nicht in ausreichendem Maße elaboriert, um hierauf basierend Hypothesen formulieren und diese anschließend einer Signifikanzprüfung unterziehen zu kőnnen. Vgl. Bortz/Döring (2006), S. 50-52 und 356f.

In diesem Zusammenhang wird von der hypothesenerkundenden oder induktiven Funktion empirischer Forschung gesprochen, wenn aufgrund eines zuvor geringen Wissensstands bezuglich des relevanten Problemfeldes eine Hypothese das Resultat einer Untersuchung bildet. Hingegen liegt die hypothesenprüfende oder deduktive Funktion vor, wenn eine zuvor abgeleitete Hypothese den Ausgangspunkt einer Untersuchung bildet, vgl. Bortz/Döring (2006), S. 30f. Eine erstmalig hypothesenprufende Untersuchung der Integration des Rechnungswesens im deutschsprachigen Raum bildet die Arbeit von Müller (2006).

19 Zimmerman (2001), S. 412 und 425.

20 So wird das Ausmaß der Vorteile einer Integration des Rechnungswesens deutlich höher eingestuft als das der Nachteile. Vgl. hierzu die Ausfuhrungen in Abschnitt B4.2 der vorliegenden Arbeit. 
ne Ausgestaltung und den - aus Sicht des Managements beurteilten - Erfolg der Controllerarbeit hypothesenbasiert und unter Zuhilfenahme fortgeschrittener Methoden der empirischen Sozialforschung untersucht, existiert bislang jedoch nicht.

Vor dem Hintergrund dieser Forschungslücke wird im Rahmen der vorliegenden Arbeit zum einen beabsichtigt, erste Schritte hin zu einer verstärkt explanativen empirischen Forschung $\mathrm{zu}$ unternehmen und in diesem Zuge die Auswirkungen einer Integration des Rechnungswesens auf die Controllerarbeit hypothesenbasiert zu analysieren. Gleichzeitig sollen zum anderen jedoch auch auf Basis deskriptiv-explorativer Analysen - etwa zu den Kontextfaktoren der Integration oder zur Umsetzung des Musters einer partiellen Integration des Rechnungswesens - zusätzliche Erkenntnisse innerhalb des betrachteten Themenfelds generiert werden, die als Grundlage für künftige hypothesenbasierte Untersuchungen dienen können.

Diese Zielsetzung lässt sich durch die Formulierung der zu beantwortenden Forschungsfragen konkretisieren, die den Leitfaden der empirischen Analyse bilden. In einem ersten Schritt ist es zunächst erforderlich, eine umfangreiche Bestandsaufnahme der Integration des Rechnungswesens bei den befragten Unternehmen durchzuführen, um somit zu einem tieferen Verständnis der Facetten dieses Phänomens zu gelangen. Durch ihren umfassenden Ansatz bietet die vorliegende Untersuchung diesbezüglich einen Erkenntnisgewinn gegenüber den bisherigen Studien, die eine Erfassung des Integrationsstands unternommen haben, da deren Ergebnisse teilweise

- zeitlich überholt sind,

- auf einer geringen Anzahl an Beobachtungen basieren,

- aufgrund der Auswahl der Untersuchungsobjekte keine umfassende Perspektive bieten,

- den Stand der Integration nicht detailliert genug erfassen oder

- wichtige Kontextfaktoren der Integration unberücksichtigt lassen. ${ }^{21}$

Darüber hinaus bilden die im Zuge der Bestandsaufnahme zu ermittelnden Integrationsintensitäten des Rechnungswesens die Grundlage für die weiteren Analysen der vorliegenden Arbeit. Somit kann die folgende Forschungsfrage formuliert werden:

$21 \mathrm{Zu}$ den bisherigen empirischen Untersuchungen vgl. die Ausführungen in Abschnitt B4 der vorliegenden Arbeit. 
Forschungsfrage 1: Wie ist der Status quo der Integration des internen und externen Rechnungswesens in deutschen Großunternehmen?

Ausgelöst durch den tiefgreifenden Wandel, dem der Finanzbereich deutscher Großunternehmen zuletzt verstärkt unterliegt, ${ }^{22}$ wird in Theorie und Unternehmenspraxis zunehmend das Rollenverständnis des Controllers diskutiert. ${ }^{23}$ Häufig wird in diesem Zusammenhang angeführt, dass die Integration des Rechnungswesens eine stärkere Ausübung der Controllerrolle als betriebswirtschaftlicher Berater des Managements unterstützt, da aufgrund der Integration eine Vielzahl ansonsten doppelt vorhandener Prozesse, Systeme und Strukturen entfallen kann und somit zeitliche Kapazitäten frei werden. ${ }^{24}$ Demnach ist es aus konzeptioneller Sicht zwar unstrittig, dass aus der Integration des Rechnungswesens Konsequenzen für die schwerpunktmäBige Ausgestaltung der Controllerarbeit hervorgehen. ${ }^{25}$ Eine explanative Überprüfung des Einflusses der Integration auf die Rollenausübung der Controller im Rahmen einer großzahligen Studie steht bislang jedoch noch aus. Die zweite Forschungsfrage lautet folglich:

Forschungsfrage 2: Welchen Einfluss hat die Integration des Rechnungswesens auf die Ausübung unterschiedlicher Controllerrollen?

Die Anforderungen an die Qualität, Flexibilität und Geschwindigkeit interner Serviceleistungen steigen zunehmend. ${ }^{26}$ Neben der Untersuchung der rollenbezogenen Ausgestaltung stellt sich daher im Zusammenhang mit der Integration des Rechnungswesens die Frage nach dem Erfolg der Controllerarbeit. Erste empirische Ergebnisse deuten darauf hin, dass eine proaktive Auseinandersetzung der Controller mit den Regelungen der externen Finanzberichterstattung positiv auf die Beurteilung des

\footnotetext{
22 Vgl. Michel (2006), S. 439ff., Daum (2008), S. 387ff. sowie Linder/Wolf (2008), S. 160ff.

23 Vgl. hierzu exemplarisch den Sammelband „Die neue Rolle des Controllers“" von Weber et al. (Hrsg.) (2008).

24 Vgl. auch im Folgenden Erichsen (2000), S. 58, Fleischer (2005), S. 195, Liedl (2005), S. 183, Michel (2006), S. 441 sowie Weißenberger (2007a), S. 44 und 218.

25 Vgl. Angelkort/Sandt/Weißenberger (2008a), S. 188.

26 Vgl. Linder/Wolf (2008), S. 160f.
} 
Controllingerfolgs seitens des Managements wirkt. ${ }^{27}$ Zudem wird sowohl aufgrund konzeptioneller Überlegungen ${ }^{28}$ als auch in empirischen Untersuchungen ${ }^{29}$ eine verbesserte Kommunikation als sehr bedeutsamer Vorteil der Integration des Rechnungswesens eingestuft. ${ }^{30}$ Im Zuge der vorliegenden Untersuchung kommt folglich der expliziten Berücksichtigung der Funktion von Finanzinformationen des Rechnungswesens als Sprache bzw. Kommunikationsmedium eine hohe Bedeutung zu. In diesem Zusammenhang merkt Bloomfield (2008) kritisch an:

„Introductory accounting textbooks often assert that 'accounting is the language of business', but researchers rarely act as if it is true. " 31

Vor diesem Hintergrund ist innerhalb der empirischen Analyse zu untersuchen, inwieweit die Integration des Rechnungswesens - unter Berücksichtigung der Eigenschaft der Finanzinformationen des Rechnungswesens als Kommunikationsmedium den seitens des Managements beurteilten Erfolg der Controllerarbeit beeinflusst. Hierdurch können Erkenntnisse darüber gewonnen werden, inwiefern die Integration des Rechnungswesens die Zielsetzung der Controller unterstützt, Mitverantwortung für die Erreichung der Unternehmensziele zu übernehmen. ${ }^{32}$ Aus diesen Überlegungen ergibt sich die dritte Forschungsfrage:

Forschungsfrage 3: Welchen Einfluss hat die Integration des Rechnungswesens auf den Erfolg der Controllerarbeit und welche Bedeutung kommt der Einheitlichkeit der Finanzsprache in diesem Zusammenhang zu?

Vgl. Angelkort/Sandt/Weißenberger (2008a), S. 191.

Vgl. Wagenhofer (2006), S. 13, Weißenberger/Angelkort (2007a), S. 426 sowie Weißenberger (2007b), S. 15.

Vgl. Hoke (2001), S. 155, Haring/Prantner (2005), S. 152 sowie Weißenberger/Angelkort (2007b), S. 29ff.

Wie in den obigen Ausführungen bereits aufgezeigt wurde, stellte der Wunsch nach einer einheitlichen und transparenten Finanzsprache den Ausgangspunkt der Neuorientierung des internen Rechnungswesens bei der Siemens AG - und somit gleichzeitig der Integrationsdiskussion - dar. Bloomfield (2008), S. 433.

Diese Zielsetzung entspricht dem Controllerleitbild der International Group of Controlling (IGC), wonach Controller den Management-Prozess der Zielfindung, Planung und Steuerung gestalten und begleiten und damit Mitverantwortung für die Zielerreichung tragen. Vgl. hierzu die Ausführungen in Abschnitt B1.2 der vorliegenden Arbeit. 
Die Beantwortung der hier abgeleiteten Forschungsfragen soll dazu beitragen, die aufgezeigte Forschungslücke im Themenfeld der Integration des internen und externen Rechnungswesens zu schließen.

\section{Wissenschaftstheoretische Einordnung und Gang der Arbeit}

Für die Analyse der Integration des Rechnungswesens kommen gemäß der Systematisierung von Grochla (1976) grundsätzlich drei verschiedene Forschungsstrategien in Frage. Unterschieden wird hier zwischen

- der sachlich-analytischen Forschungsstrategie,

- der empirischen Forschungsstrategie sowie

- der formal-analytischen Forschungsstrategie. ${ }^{33}$

Die sachlich-analytische Forschungsstrategie strebt eine Durchleuchtung komplexer Zusammenhänge sowie die Erarbeitung von Handlungsgrundlagen an, ohne jedoch eine eigene empirische Überprüfung der von ihr generierten Aussagen zu bezwecken (spekulatives Element). Hingegen ist die empirische Forschungsstrategie durch die Zielsetzung einer systematischen Erfahrungsgewinnung charakterisiert. Hierzu sollen konkrete Aussagen über die Realität mithilfe von Methoden der empirischen Sozialforschung bzw. der induktiven Statistik überprüft werden. Bei der formal-analytischen Forschungsstrategie geht es schließlich um eine Vereinfachung bzw. abstrakte Beschreibung von Problemstrukturen anhand einer formalen Modellbildung.

Wie aus den zuvor abgeleiteten Forschungsfragen bereits ersichtlich ist, wird für die vorliegende Untersuchung auf die empirische Forschungsstrategie zurückgegriffen. Da mit der Beantwortung dieser Forschungsfragen ein pragmatisches Wissenschaftsziel verfolgt wird, ist die vorliegende Arbeit schwerpunktmäßig im Bereich der Wirtschaftstechnologie - diese stellt ein System von anwendungsbezogenen, aber allgemeingültigen Ziel/Mittel-Aussagen dar - anzusiedeln. ${ }^{34}$ Struktur.

Die vorliegende Arbeit folgt der in Abbildung 2 dargestellten inhaltlichen

33 Vgl. auch im Folgenden Grochla (1976), S. 634.

34 Vgl. Chmielewicz (1994), S. 8-15. 


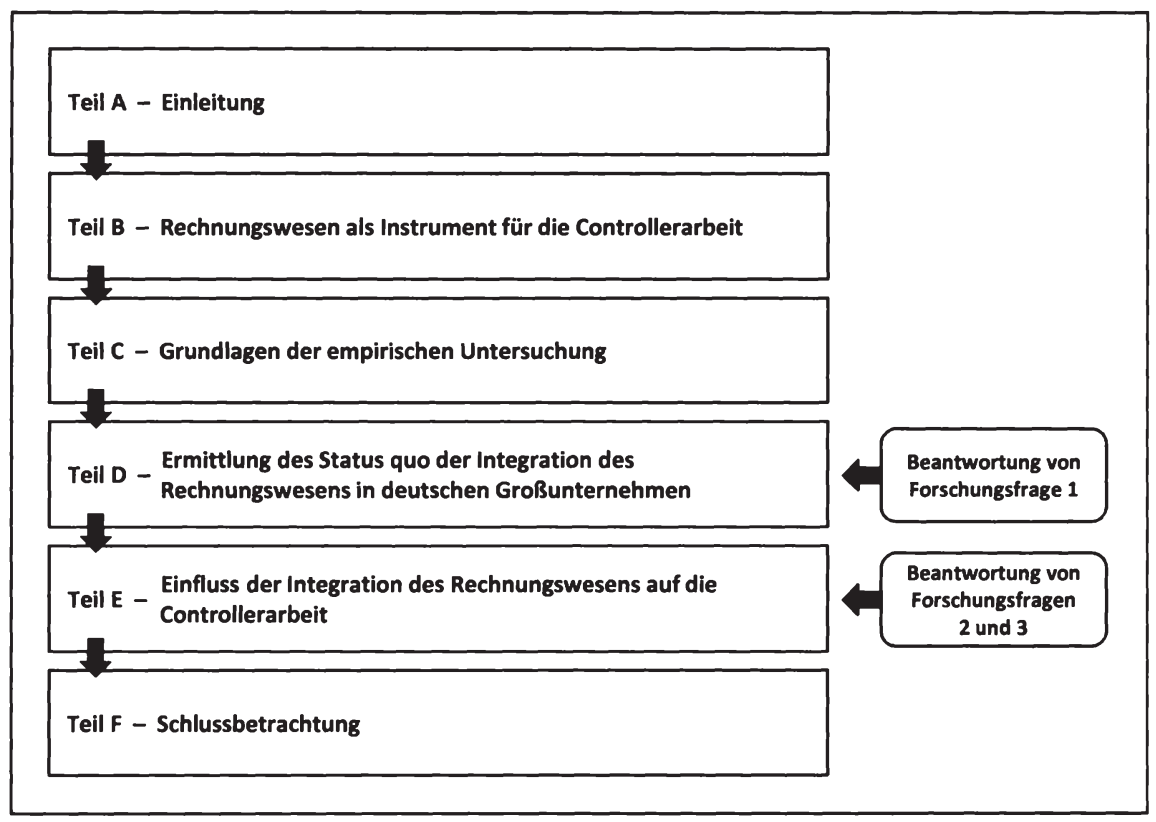

Abbildung 2: Gang der Arbeit

Im Anschluss an den einfuihrenden Teil A wird in Teil B die Funktion des Rechnungswesens als Instrument für die Controllerarbeit dargestellt. Zunächst wird dazu das der Arbeit zu Grunde liegende Controllingverständnis abgegrenzt und das House of Controlling als konzeptioneller Bezugsrahmen der Controllerarbeit vorgestellt. Neben einer kurzen Abgrenzung des Rechnungswesensbegriffs werden anschließend mit den Aufgaben und Teilbereichen die wichtigsten Grundlagen des - im deutschsprachigen Raum traditionell zweigeteilten - betrieblichen Rechnungswesens dargelegt. Im nächsten Schritt werden das Konzept eines integrierten Rechnungswesens sowie das im Schrifttum häufig für konzeptionell vorteilhaft erachtete Muster einer partiellen Integration des Rechnungswesens als Ansatzpunkte für eine Vereinheitlichung von internem und externem Rechnungswesen erörtert. Im letzten Abschnitt von Teil B erfolgt zudem eine Bestandsaufnahme der empirischen Forschung im Themenfeld der Integration des Rechnungswesens. Dazu wird zunächst ein Überblick über die diesbezüglich im deutschsprachigen Raum durchgeführten Studien gegeben. AnschlieBend werden die zentralen hieraus gewonnenen Untersuchungsergebnisse aufgezeigt. 
Eine Darstellung der Grundlagen der eigenen empirischen Untersuchung erfolgt in Teil C. Hierzu wird zunächst das dyadische Forschungsdesign vorgestellt, welches eine Befragung sowohl des Leiters Controlling als auch eines ergebnisverantwortlichen Managers in jedem Unternehmen vorsieht. Im nächsten Schritt wird die Vorgehensweise der unter den 1.500 umsatzstärksten Unternehmen in Deutschland durchgeführten Datenerhebung skizziert. Zudem wird die hierdurch geschaffene Datengrundlage hinsichtlich der erzielten Rücklaufquoten sowie der Stichprobenmerkmale charakterisiert. Anschließend erfolgt eine Darstellung der im Zuge der Untersuchung verwendeten statistischen Analyseverfahren.

In Teil D wird auf Basis einer deskriptiv-explorativen Vorgehensweise der Status quo der Integration des internen und externen Rechnungswesens in deutschen Großunternehmen empirisch ermittelt und somit die erste Forschungsfrage beantwortet. Als erstes werden in diesem Zuge verschiedene integrationsrelevante Aspekte der Controllerarbeit erfasst und anschließend $\mathrm{zu}$ Integrationsindizes verdichtet, die die Integrationsintensität innerhalb der verschiedenen Aufgabenfelder der Controllerarbeit widerspiegeln. Auf Basis dieser Indizes werden zudem Integrationsprofile aufgestellt, um den Einfluss verschiedener Kontextfaktoren auf die Ausprägung der Integration des Rechnungswesens näher zu beleuchten. Im letzten Schritt wird exemplarisch die Identifizierung und Quantifizierung des Musters einer partiellen Integration des Rechungswesens unternommen sowie der Zusammenhang zu verschiedenen Kontextfaktoren hergestellt. Mit einem kurzen Zwischenfazit zum Status quo der Integration endet dieser Teil der Arbeit.

In Teil $\mathbf{E}$ wird als erstes in deskriptiv-explorativer Weise die Ausübung verschiedener Controllerrollen empirisch erfasst, wobei zwischen der bisher realisierten und der seitens der Controller zukünftig angestrebten Rollenausübung differenziert wird. Hierauf aufbauend erfolgt im nächsten Schritt eine explanative Analyse des Einflusses der Integration des Rechnungswesens auf die rollenbezogene Ausgestaltung der Controllerarbeit und somit die Beantwortung der zweiten Forschungsfrage. Anschließend erfolgt unter Zuhilfenahme der Kovarianzstrukturanalyse die Beantwortung der dritten Forschungsfrage. Zunächst werden hierzu auf theoretisch-konzeptioneller Basis die Hypothesen hinsichtlich des Einflusses der Integration des Rechnungswesens auf den Erfolg der Controllerarbeit abgeleitet, wobei die Funktion von Finanzinformationen des Rechnungswesens als Sprache bzw. Kommunikationsmedium explizit berücksich- 
tigt wird. Danach erfolgt die Operationalisierung der innerhalb des Kovarianzstrukturmodells berücksichtigten latenten Untersuchungsvariablen. Im Rahmen der hierauf folgenden Darstellung der Analyseergebnisse wird als erstes die Güte der Konstruktmessung belegt. Im nächsten Schritt werden die einzelnen Hypothesen des Kovarianzstrukturmodells überprüft, wobei zur genaueren Untersuchung der Dependenzbeziehungen eine Effektzerlegung in direkte, indirekte und Gesamteffekte sowie eine aktionsfeldbezogene Detailanalyse vorgenommen werden. Des Weiteren erfolgt eine explorativ angelegte, kovarianzstrukturanalytische Mehrgruppenanalyse. Deren Ziel ist es, einen eventuell vorhandenen moderierenden Effekt der Gruppenzugehörigkeit der Antwortenden auf die Dependenzbeziehungen zu identifizieren und somit herauszufinden, ob es zwischen Controllern und Managern zu systematischen Wahrnehmungsunterschieden kommt. Im letzten Schritt werden die Implikationen der in Teil E gewonnenen empirischen Analyseergebnisse diskutiert.

Teil F schließt die Arbeit nach einer Zusammenfassung der zentralen Untersuchungsergebnisse mit einer kritischen Würdigung und einem Ausblick auf die weitere Forschung ab. 


\section{B Rechnungswesen als Instrument für die Controller- arbeit}

„Jeder hat seine eigenen Vorstellungen darüber, was Controlling bedeutet oder bedeuten sollte, doch jeder meint etwas anderes, wenn er von Controlling spricht."

Preißler (2007), S. 14

\section{Controlling als spezielle Führungsfunktion}

\subsection{Controllingverständnis}

In den vergangenen Jahrzehnten hat sich Controlling innerhalb der Unternehmenspraxis als Führungsfunktion etabliert. ${ }^{35}$ Unter Controlling versteht man dabei im deutschsprachigen Raum den

„gesamten Prozeß der Zielfestlegung, der Planung und der Steuerung im finanz- und im leistungswirtschaftlichen Bereich. "36

Zwar sind die damit verbundenen Aufgaben nicht zwangsläufig an die Person des Controllers gebunden - im Vordergrund stehen hier vielmehr eine ziel- und planungsorientierte Gestaltung der Führungsprozesse oder eine dezentrale Organisation der Verantwortlichkeiten im Management. Mit zunehmender Unternehmensgröße ist für eine controllinggerechte Führung jedoch die Einrichtung spezialisierter Controllerstellen erforderlich. Als Stelleninhaber übernimmt der Controller durch die Bereitstellung betriebswirtschaftlicher Kennzahlen in erster Linie die Funktion eines ökonomischen Navigators und Sparringspartner für das Management. Hierdurch wird die Gesamtführungsaufgabe insofern aufgespalten, als dass der Manager die realwirtschaftlichen Sachprobleme, der Controller hingegen die damit verbundenen Mess- bzw. Bewertungsprobleme löst. Der Manager wird durch diese Aufgabenteilung entlastet, da ihm die komplexe Abbildung des Unternehmensgeschehens in betriebswirtschaftlich 
aussagekräftigen Steuerungsgrößen durch den Controller abgenommen wird. Während sich der Manager folglich auf die inhaltliche Konkretisierung von Prozessen und Maßnahmen konzentrieren kann, entwickelt sich der Controller zum Kennzahlenspezialisten. $^{37}$

Im Falle weitreichender dezentraler Entscheidungskompetenzen müssen Controller in diesem Zusammenhang zum einen dafür sorgen, dass für die vorliegenden Entscheidungsprobleme durch Verwendung geeigneter Mess- und Bewertungsinstrumente eine möglichst gute Lösung gefunden wird ('decision-making'). Zum anderen müssen sie sicherstellen, dass unter Berücksichtigung von bestehenden Informationsasymmetrien und Interessenkonflikten adäquate dezentrale Steuerungsimpulse gegeben werden ('decision-influencing'). ${ }^{38}$

\subsection{House of Controlling als konzeptioneller Bezugsrahmen der Controllerarbeit}

Die Umsetzung des soeben skizzierten Controllingverständnisses in die praktische Controllerarbeit ${ }^{39}$ kann über das in Abbildung 3 dargestellte House of Controlling systematisiert werden.

So wird die übergeordnete Zielsetzung der Controllerarbeit als 'Dach' durch das Controllerleitbild der International Group of Controlling (IGC) zusammengefasst, wonach Controller den Management-Prozess der Zielfindung, Planung und Steuerung gestalten und begleiten und damit Mitverantwortung für die Zielerreichung tragen. ${ }^{40}$

Vgl. Weißenberger (2002), S. 398f., Weißenberger (2007a), S. 30ff., Weißenberger/Angelkort (2007a), S. 412 sowie Angelkort/Sandt/Weißenberger (2008b), S. 62. Der Begriff 'Controller' ist hierbei institutionell geprägt und bezeichnet den Aufgabentrăger bzw. Stelleninhaber, der die Controllingaufgaben wahrnimmt. Vgl. hierzu auch Weber/Schäffer (2008), S. 1 sowie Horváth (2009), S. 125.

Vgl. Weißenberger (2004), S. 296 sowie Ewert/Wagenhofer (2008), S. $6 \mathrm{ff}$.

39 Der Begriff 'Controllerarbeit' beschreibt das von Controllern wahrgenommene Aufgabenbündel und wird auch als 'Controllership' bezeichnet, vgl. Angelkort/Sandt/Weißenberger (2008b), S. 62 sowie Weber/Schäffer (2008), S. 1.

Die International Group of Controlling ist eine internationale berufsständische Dachorganisation, deren Mitglieder Controlling in der praktischen Anwendung und Weiterentwicklung fördern wollen. Für das ausfuhrliche Controllerleitbild der IGC vgl. International Group of Controlling (2009). 


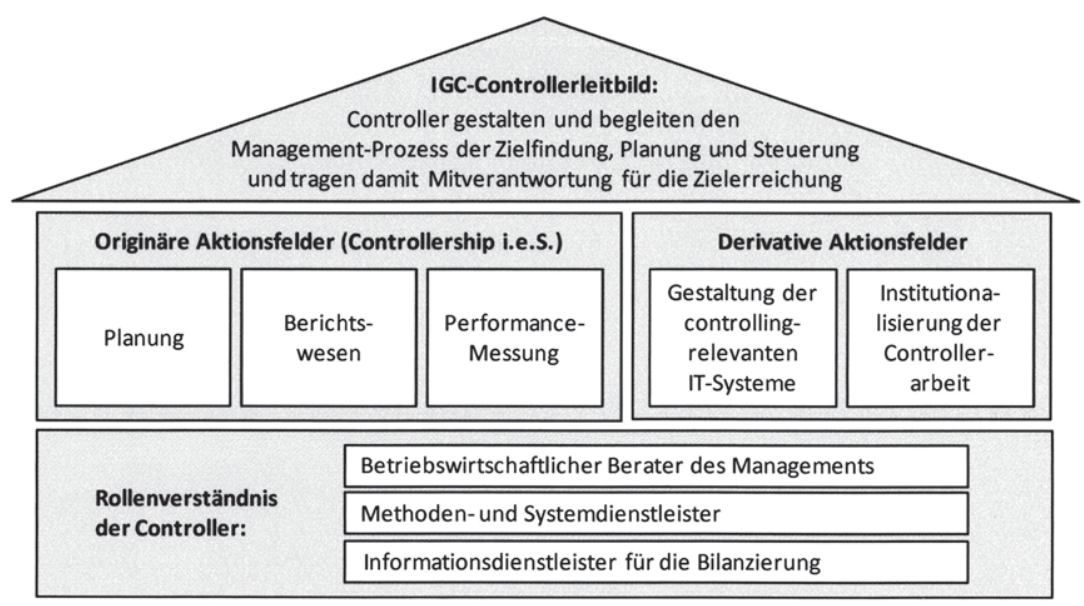

Abbildung 3: House of Controlling ${ }^{41}$

Das 'Fundament' des House of Controlling wird durch das Rollenverständnis der Controller gebildet. Für eine controllinggerechte Führung müssen Controller demnach drei verschiedene Rollen wahrnehmen: ${ }^{42}$

- In ihrer Rolle als betriebswirtschaftlicher Berater sind Controller die Sparringspartner des Managements und tragen als Navigator zur Zielerreichung bei.

- Als Methoden- und Systemdienstleister stellen Controller die im Rahmen der betriebswirtschaftlichen Beratung benötigten Steuerungsinformationen bereit.

- Insbesondere im Kontext der IFRS kommt den Controllern neben den zwei bestehenden Rollen zusätzlich die Rolle als Informationsdienstleister für die Bilanzierung zu, durch die sie Mitverantwortung für die externe Finanzberichterstattung übernehmen.

41 Eigene Darstellung in Anlehnung an Weißenberger (2006a), S. 33 sowie Weißenberger (2007a), S. 40.

42 Vgl. auch im Folgenden Weißenberger (2006a), S. 19f. und 32, Weißenberger (2007a), S. 35 und 41 sowie Weißenberger (2007c), S. 324. 
Die inhaltliche Umsetzung der Controllerarbeit erfolgt durch die drei originären Aktionsfelder, die Dach und Fundament des House of Controlling verbinden und somit die 'Säulen' einer ziel- bzw. ergebnisorientierten Unternehmensführung darstellen: ${ }^{43}$

- Die Planung umfasst neben der laufenden operativen, taktischen und strategischen Unternehmensplanung sowohl die fallweise erforderliche Projektplanung als auch die Ermittlung von Prognose- bzw. Forecast-Größen. Neben dem Planungsmanagement, d.h. der Gewährleistung der erforderlichen zeitlichen, hierarchischen und inhaltlichen Verzahnung sämtlicher Planungsebenen und -größen, sind die Controller methodisch insbesondere für die Formalzielplanung, d.h. die Budgetierung sowie die Bilanz- und Finanzplanung, verantwortlich.

- Das Berichtswesen schließt neben der laufenden und fallweisen Informationsbereitstellung im Ist (Monatsberichterstattung, Sonderauswertungen) diejenigen Analysen mit ein, die sich aus Soll-Ist-Abweichungen bzw. Abweichungen zwischen den Plan- und Forecast-Größen ergeben (Kontrollen). Dabei tragen Controller die Verantwortung sowohl für die inhaltliche als auch für die formale Gestaltung und Informationsqualität des internen Berichtswesens.

- Die Performance-Messung bildet die Grundlage für die dezentrale Steuerung, da sie die Erfolgsbeiträge von Prozessen, Produkten und Bereichen aufzeigt. Zur Sicherstellung einer koordinierten und zielführenden dezentralen Leistungserstellung ist das jeweilige eigenständige Handeln auf verschiedenen Managementebenen durch adäquate Kennzahlen abzubilden. In diesem Zusammenhang unterstützen Controller das zentrale Management bei der Auswahl und Implementierung geeigneter Steuerungsgrößen.

Kontrolle, die in der Literatur vielfach als weiteres bedeutsames Aktionsfeld der Controllerarbeit eingestuft wird, ${ }^{44}$ ist hierbei sowohl innerhalb der Planung zur Verbesserung der informationalen Grundlage zukünftiger Entscheidungen (feed-forwardFunktion bzw. Entscheidungsunterstützung) als auch innerhalb der Performance- 
Messung zur Willensdurchsetzung der Instanz gegenüber dezentralen Entscheidungsträgern (feed-back-Funktion bzw. Verhaltenssteuerung) enthalten. ${ }^{45}$

Zur Realisierung der aus den drei originären Aktionsfeldern resultierenden Aufgaben ist die Unterstützungsleistung der beiden so genannten derivativen Aktionsfelder erforderlich: ${ }^{46}$

- Im Rahmen der Gestaltung der controllingrelevanten IT-Systeme müssen Controller sicherstellen, dass für Zwecke der Planung, des Berichtswesens und der Performance-Messung eine controllinggerechte Infrastruktur an Informationsund Kommunikationstechnologien bereitsteht. $\mathrm{Zu}$ diesen Vorsystemen zählen unter anderem buchhalterische Datenquellen, wie z.B. das Materialwirtschaftssystem, die Debitoren- und Kreditorenbuchhaltung, die Personalbuchhaltung sowie die Anlagenbuchhaltung - häufig umgesetzt in Form von Enterprise Resource Planning (ERP)-Systemen. Darüber hinaus müssen Controller die Datenbanken bzw. Data Warehouse-Systeme gestalten, aus denen die für die Controllingaufgaben notwendigen Buchungs-, Bewertungs-, Markt- oder Stammdaten extrahiert und beispielsweise in Führungsinformationssysteme übertragen werden. Nicht im Aufgabenbereich der Controller liegt hingegen die technische Verantwortung bezüglich der IT-Systeme, da diese in der Regel an eine eigens hierfür eingerichtete Stelle bzw. Fachabteilung übertragen wird.

- Die fachliche Institutionalisierung der Controllerarbeit umfasst neben der ablauforganisatorischen Gestaltung von Controllingprozessen ebenfalls die Strukturierung der Aufbauorganisation des Controllerbereichs sowie der Zusammenarbeit mit anderen Funktionen des Finanzbereichs, wie z.B. dem externen Rechnungswesen, dem Risikomanagement, der Revision oder dem Treasury. Zudem gehört es im Rahmen der Institutionalisierung zu den Aufgaben der Controller, sämtliche erforderlichen Maßnahmen hinsichtlich Personalauswahl und -einsatz für Controlleraufgaben sowie Aus- und Weiterbildung im Controlling zu treffen.

45 Vgl. zu den verschiedenen Funktionen der Kontrolle Weißenberger (2004), S. 296.

46 Vgl. auch im Folgenden Weißenberger (2006a), S. 20, Weißenberger (2007a), S. 36f., Angelkort/ Sandt/Weißenberger (2008b), S. 66 sowie Weißenberger (2008a), Rz. 11. 
Die hohe Praxisrelevanz der Systematisierung des House of Controlling zeigt sich unter anderem darin, dass diese im Controlling des Deutsche Bahn-Konzerns Anwendung gefunden hat. ${ }^{47}$

Für die vorliegende empirische Untersuchung ist das House of Controlling zudem konzeptionell von besonderer Bedeutung, da seine drei Komponenten - also Dach, Fundament und Säulen - die Struktur der drei zu beantwortenden Forschungsfragen repräsentieren bzw. widerspiegeln. ${ }^{48}$

\section{Grundlagen des betrieblichen Rechnungswesens im deutsch- sprachigen Raum}

\subsection{Begriff und Aufgabe des Rechnungswesens}

Das betriebliche Rechnungswesen bildet einen zentralen Bestandteil des Informationssystems eines Unternehmens. ${ }^{49}$ Im Gegensatz zum Terminus 'Rechnungslegung', der ausschließlich auf die Zahlenwerke der Finanz- bzw. Managementberichte abstellt, umfasst der hier verwendete Begriff des Rechnungswesens zusätzlich auch organisatorische Aspekte und ist somit weiter gefasst. ${ }^{50}$ Die Aufgabe des Rechnungswesens liegt in der

47 Vgl. Lutz (2007), S. 104.

48 So erfolgt die detaillierte Ermittlung des Status quo der Integration des Rechnungswesens (Forschungsfrage 1) bei den betrachteten Unternehmen untergliedert nach den fünf Aktionsfeldern der Controllerarbeit. In Forschungsfrage 2 wird der Einfluss der Integration des Rechnungswesens auf die Ausübung der verschiedenen Controllerrollen untersucht. Die Analyse der Auswirkungen einer Integration des Rechnungswesens auf den Erfolg der Controllerarbeit (Forschungsfrage 3) zeigt schließlich auf, wie gut es den Controllern gelingt, Mitverantwortung für die Zielerreichung im Sinne des IGC-Controllerleitbilds zu tragen. Vgl. hierzu auch Abbildung 7 in Abschnitt C1.1 der vorliegenden Arbeit. Vgl. Eisele (2002), S. 3. Der Zusatz 'betrieblich' - bzw. teilweise auch 'betriebswirtschaftlich' grenzt das Rechnungswesen hierbei von gesamtwirtschaftlichen Rechnungen ab, vgl. Hoke (2001), S. 6. Im Rahmen der vorliegenden Arbeit ist mit Rechnungswesen stets das betriebliche Rechnungswesen gemeint, wobei jedoch auf die Verwendung dieses Zusatzes verzichtet wird.

Vgl. Simons/Weißenberger (2009a), S. 391. Mitunter werden beide Begriffe jedoch synonym verwendet, vgl. Weißenberger (2003), S. 87 m.w.N. Für eine ausführliche Lehrbuchanalyse bezüglich der Begriffsverwendung und Definition des Rechnungswesens vgl. zudem Schaier (2007), S. 80-83. 
„quantitativen wertmäßigen Abbildung von Wirtschaftsgütern zum Zwecke der Wertelenkung von Güterbeständen und -bewegungen “51.

Das Rechnungswesen liefert hierdurch in zweckdienlicher Form Informationen als Entscheidungshilfen für die Unternehmensleitung und übernimmt somit eine Kommunikationsfunktion. ${ }^{52}$ Umgesetzt wird diese Funktion durch die Erstellung von

„Rechnungen, die mehrdimensionale, reale einzelwirtschaftliche Sachverhalte unter Verwendung des Maßstabes Geld eindimensional in die Sprachebene abbilden und dadurch zur Komplexitätsreduktion führen “"53.

Folglich kann das Rechnungswesen auch als (Finanz-)Sprache bezeichnet werden, mit deren Hilfe unter anderem Ziele kommuniziert und Entscheidungsverhalten beeinflusst werden können. ${ }^{54}$ Die Notwendigkeit der monetären Abbildung realwirtschaftlicher Sachverhalte ergibt sich dabei - neben den gesetzlichen Erfordernissen - insbesondere aufgrund wachsender Unternehmensgrößen und der damit einhergehenden Dezentralisierung, d.h. der zunehmenden Verlagerung operativer Aufgaben in eigenständige Unternehmensbereiche. ${ }^{55}$ Die Unternehmensleitung kann in einem solchen Umfeld die notwendigen Informationen bezüglich der realwirtschaftlichen Leistungserstellung nicht mehr unmittelbar beobachten. Vielmehr muss die unmittelbare, persönliche Wahrnehmung der relevanten Sachverhalte stattdessen durch die vom Rechnungswesen erfassten und aufbereiteten monetären Informationen ersetzt werden. Iletschko (1955) spricht in diesem Zusammenhang von der Notwendigkeit einer Information ,nicht nach Besicht, sondern nach Bericht. ${ }^{656}$ An dieser Stelle wird zudem die enge Verknüpfung deutlich, die im deutschsprachigen Raum zwischen Rechnungswesen

$51 \quad$ Chmielewicz (1982), S. 13.

52 Vgl. Eisele (2002), S. 3.

53 Goetzke (1979), S. 518.

54 Zur Funktion des Rechnungswesens als Kommunikationsmedium bzw. Sprache vgl. Belkaoui (1980), Otley (1980), S. 242, Boland/Pondy (1983), S. 228, Lavoie (1987), Belkaoui (1989), Pfaff/Weber (1998), Evans (2004), Schaier (2007), S. 188ff., Bloomfield (2008) sowie Simons/ Weißenberger (2009b), S. 450.

55 Vgl. auch im Folgenden Weißenberger (2003), S. 85f. Ein weiterer Grund ist zudem darin zu sehen, dass eine gesamthafte Betrachtung von Ressourcen, Prozessen, Strukturen oder Outputs im Unternehmen aufgrund der realwirtschaftichen Vielfalt i.d.R. ausschließlich durch die Umrechnung in Geldgrößen moglich ist.

56 Illetschko (1955), S. 31. 
und Controlling besteht. So bildet das Rechnungswesen ein wichtiges Instrument für die Controllerarbeit. ${ }^{57}$

\subsection{Traditionelle Teilbereiche des Rechnungswesens}

Das Rechnungswesen lässt sich gemäß seiner primären Adressaten in die Teilbereiche des externen und internen Rechnungswesens untergliedern (vgl. Tabelle 1).

\begin{tabular}{|c|c|c|c|c|c|c|}
\hline & \multicolumn{3}{|c|}{ Externes Rechnungswesen } & \multicolumn{3}{|c|}{ Internes Rechnungswesen } \\
\hline $\begin{array}{l}\text { Adres- } \\
\text { saten }\end{array}$ & \multicolumn{3}{|c|}{$\begin{array}{c}\text { Externe Adressaten } \\
\text { (Fiskus, Kapitalgeber, Geschäftspartner, } \\
\text { sonstige interessierte Öffentlichkeit) }\end{array}$} & \multicolumn{3}{|c|}{$\begin{array}{l}\text { Interne Adressaten } \\
\text { (Unternehmensleitung, Führungskräfte) }\end{array}$} \\
\hline $\begin{array}{l}\text { Rechen- } \\
\text { werke }\end{array}$ & Bilanz & GuV & $\begin{array}{l}\text { Kapitalfluss- } \\
\text { rechnung }\end{array}$ & $\begin{array}{c}\text { Kosten- und } \\
\text { Erlösrechnung }\end{array}$ & $\begin{array}{l}\text { Finanz- } \\
\text { planung }\end{array}$ & $\begin{array}{c}\text { Investitions- } \\
\text { rechnung }\end{array}$ \\
\hline $\begin{array}{l}\text { Rechen- } \\
\text { größen }\end{array}$ & $\begin{array}{l}\text { Vermögen, } \\
\text { Schulden }\end{array}$ & $\begin{array}{l}\text { Aufwendun- } \\
\text { gen, Erträge }\end{array}$ & \begin{tabular}{|} 
Einzahlungen, \\
Auszahlungen
\end{tabular} & $\begin{array}{l}\text { Kosten, } \\
\text { Erlöse }\end{array}$ & $\begin{array}{l}\text { Einzahl } \\
\text { Auszah }\end{array}$ & $\begin{array}{l}\text { lungen, } \\
\text { lungen }\end{array}$ \\
\hline $\begin{array}{l}\text { Zeitlicher } \\
\text { Bezug }\end{array}$ & \multicolumn{4}{|c|}{ Laufend, einperiodig } & $\begin{array}{c}\text { Laufend, } \\
\text { ein- oder } \\
\text { mehrperiodig }\end{array}$ & $\begin{array}{l}\text { Fallweise, } \\
\text { i.d.R. } \\
\text { mehrperiodig }\end{array}$ \\
\hline $\begin{array}{l}\text { Normie- } \\
\text { rung }\end{array}$ & \multicolumn{3}{|c|}{ Gesetzliche Vorschriften } & \multicolumn{3}{|c|}{$\begin{array}{l}\text { Unternehmensindividuelle } \\
\text { Gestaltungsfreiheit }\end{array}$} \\
\hline $\begin{array}{l}\text { Rechen- } \\
\text { zwecke }\end{array}$ & \multicolumn{3}{|c|}{$\begin{array}{l}\text { Zahlungsbemessung, } \\
\text { Information }\end{array}$} & \multicolumn{3}{|c|}{ Planung, Kontrolle, Dokumentation } \\
\hline
\end{tabular}

Tabelle 1: Gegenüberstellung von externem und internem Rechnungswesen ${ }^{58}$

Das externe Rechnungswesen richtet sich in erster Linie an Unternehmensexterne, wie z.B. den Fiskus, die Eigen- und Fremdkapitalgeber, Geschäftspartner wie Kunden oder Lieferanten sowie die sonstige interessierte Öffentlichkeit. ${ }^{59}$ Die zentralen Rechenwerke des externen Rechnungswesens sind die Bilanz, die Gewinn- und Verlustrechnung $(\mathrm{GuV})$ sowie die Kapitalflussrechnung. ${ }^{60}$ Als stichtagsbezogene Bestandsrechnung stellt die Bilanz das Vermögen (Aktivseite: Kapitalverwendung) dem Kapital (Passivseite: Kapitalherkunft) gegenüber, wobei sich das Eigenkapital als Saldo aus

Vgl. Weißenberger (2004), S. 293. Hahn/Hungenberg (2001), S. 272, stellen diesbezüglich fest: „Primäre Basis des Controlling bildet [...] das Zahlenwerk des Rechnungs- und Finanzwesens“. Eigene Darstellung in Anlehnung an Weißenberger (1997), S. 28, Küting/Lorson (1998), S. 484 sowie Weißenberger (2003), S. 90.

59 Vgl. Schneider (1997), S. 29, Weißenberger (1997), S. 28, Küpper (1998), S. 151, Klein (1999), S. 11, Weißenberger (2003), S. 90, Ewert/Wagenhofer (2008), S. 3, Pellens et al. (2008), S. 4f. sowie Becker/Baltzer (2009a), S. 173f. 
Vermögen und Schulden bzw. Fremdkapital ergibt. ${ }^{61}$ Die GuV ermittelt anhand von Stromgrößen, den so genannten Aufwendungen und Erträgen, den Jahresüberschuss bzw. -fehlbetrag einer Periode. ${ }^{62}$ Dieser entspricht als Konsequenz der doppelten Buchführung wiederum der Veränderung des bilanziellen Eigenkapitals, sofern innerhalb der betrachteten Periode keine Einlagen oder Entnahmen von Eigenkapital vorgenommen wurden. ${ }^{63}$ Im Rahmen der Kapitalflussrechnung - ebenfalls eine StromgröBenrechnung - werden die Ein- und Auszahlungen einer Periode erfasst, deren Saldo die Veränderung des Bestands an liquiden Mitteln anzeigt. ${ }^{64}$ Bilanz, GuV und Kapitalflussrechnung stellen jeweils eine laufende und einperiodige Form der Rechnung dar. ${ }^{65}$ Die Gestaltung der Rechenwerke des externen Rechnungswesens unterliegt gesetzlichen Normen, so z.B. dem Handels- und Steuerrecht, den Deutschen Rechnungslegungs Standards (DRS) - diese stehen im Rang von Grundsätzen ordnungsmäßiger Buchführung - sowie den International Financial Reporting Standards (IFRS). ${ }^{66}$ Das externe Rechnungswesen dient dabei vor allem der Erfüllung zweier Rechnungszwecke. ${ }^{67}$ Zum einen bildet der handelsrechtliche Einzelabschluss im Rahmen der Zahlungsbemessungsfunktion durch die vorsichtige - d.h. vom Gläubigerschutzgedanken geleitete - Ermittlung eines ausschüttungs- und besteuerungsfähigen Gewinns die Grundlage der Festlegung der Dividenden- und Steuerzahlungen. Zum anderen sollen den Adressaten möglichst verlässliche und aussagekräftige Informationen über die Vermögens-, Finanz- und Ertragslage des Unternehmens bereitgestellt werden. Anders als im Falle der Zahlungsbemessungsfunktion sind für diese Informationsfunktion grundsätzlich sowohl Einzel- als auch Konzernabschluss relevant. Während die Informationsfunktion des handelsrechtlichen Jahresabschlusses aufgrund der starken Ausrichtung auf den Gläubigerschutz sowie der engen Verflechtung von Handels-, Gesellschafts- und Steuerrecht jedoch zurückgedrängt wurde, ${ }^{68}$ stellt die Vermittlung ent-

61 Vgl. Weißenberger (1997), S. 28, Pellens et al. (2008), S. 3 sowie Weber/Kabst (2009), S. 329.

62 Vgl. Weißenberger (1997), S. 28, Pellens et al. (2008), S. 3 sowie Weber/Kabst (2009), S. 335.

63 Vgl. Becker/Baltzer (2009a), S. 175.

64 Vgl. Pellens et al. (2008), S. 3.

$65 \mathrm{Vgl}$. Weißenberger (2003), S. 92.

66 Vgl. Weißenberger (2003), S. 90ff. sowie Becker/Baltzer (2009b), S. $226 \mathrm{f}$.

67 Vgl. auch im Folgenden Coenenberg (1995), S. 2078, Weißenberger (1997), S. 29, Küpper (1998), S. 151, Kümpel (2002), S. 343 sowie Pellens et al. (2008), S. 12-14.

68 Vgl. Simons/Weißenberger (2009a), S. 393. 
scheidungsrelevanter Informationen für Investoren die zentrale Zielsetzung innerhalb des Normensystems der IFRS dar. ${ }^{69}$

Im Gegensatz zum externen Rechnungswesen liefert das interne Rechnungswesen Informationen für unternehmensinterne Akteure, die mit Entscheidungs- und Weisungskompetenz ausgestattet sind (Unternehmensleitung, Führungskräfte) ${ }^{70} \mathrm{Zu}$ den Rechenwerken des internen Rechnungswesens zählen die Kosten- und Erlösrechnung, die Finanzplanung sowie die Investitionsrechnung. Als laufende und einperiodige Rechnung bildet die Kosten- und Erlösrechnung den im Zuge des betrieblichen Kombinationsprozesses notwendigen Einsatz an Produktionsfaktoren sowie die hieraus resultierenden Leistungsergebnisse monetär $a b^{71}$ Die Bewertung dieser Größen weicht jedoch von den pagatorischen - d.h. aus der Periodisierung von Zahlungen resultierenden - Wertansätzen der GuV ab, wie Abbildung 4 exemplarisch anhand der zweistufigen Abgrenzung von Aufwendungen und Kosten verdeutlicht.

\begin{tabular}{|c|c|c|c|}
\hline \multicolumn{3}{|c|}{$\begin{array}{l}\text { Aufwendungen } \\
\text { (bewerteter Verbrauch von Gütern/Dienstleistungen) }\end{array}$} & \\
\hline \multirow{5}{*}{$\begin{array}{l}\text { Neutraler Aufwand } \\
\text { (betriebs-/perioden- } \\
\text { fremd, außerordentlich) }\end{array}$} & \multicolumn{2}{|c|}{ Zweckaufwand } & \\
\hline & $\begin{array}{c}\text { Als Kosten verrechneter } \\
\text { Zweckaufwand }\end{array}$ & $\begin{array}{c}\text { Nicht als Kosten verrech- } \\
\text { neter Zweckaufwand }\end{array}$ & \\
\hline & \multirow{2}{*}{ Grundkosten } & Anderskosten* & Zusatzkosten \\
\hline & & \multicolumn{2}{|c|}{ Kalkulatorische Kosten } \\
\hline & \multicolumn{3}{|c|}{ Kosten } \\
\hline
\end{tabular}

* Die Anderskosten können größer oder kleiner als der nicht als Kosten verrechnete Zweckaufwand sein.

\section{Abbildung 4: Abgrenzung von Aufwendungen und Kosten ${ }^{72}$}

In dieser auch als 'Schmalenbach'sche Treppe' bezeichneten Systematik wird zum einen der so genannte neutrale Aufwand nicht als Kostenbestandteil berücksichtigt, da er in keinem Zusammenhang mit der Leistungserstellung der Periode steht. Zum anderen werden kalkulatorische Kosten angesetzt, entweder indem eine abweichende

69 Vgl. International Group of Controlling (Hrsg.)/Weißenberger (2006), S. 25 sowie Pellens et al. (2008), S. 112f.

70 Vgl. Schneider (1997), S. 30, Weißenberger (1997), S. 29, Küpper (1998), S. 151, Klein (1999), S. 11, Weißenberger (2003), S. 89f., Ewert/Wagenhofer (2008), S. 3 sowie Becker/Baltzer (2009a), S. 173.

71 Vgl. Weißenberger (1997), S. 29, Eisele (2002), S. 635 sowie Weber/Weißenberger (2006), S. $337 \mathrm{f}$.

72 Eigene Darstellung in Anlehnung an Eisele (2002), S. 642. 
wertmäßige Behandlung von Sachverhalten vorgenommen wird (Anderskosten, z.B. wiederbeschaffungswertbezogene Abschreibungen) oder indem ein Werteverzehr berücksichtigt wird, der sich im externen Rechnungswesen weder mengen- noch wertmäßig niederschlägt (Zusatzkosten, z.B. kalkulatorische Zinsen für das zur Verfügung gestellte Eigenkapital). ${ }^{73}$ Das Ziel dieser Anpassungen bzw. Ergänzungen ist es, die Abbildung der Faktorverbräuche bzw. Leistungsergebnisse an die internen Informationsbedürfnisse anzupassen. ${ }^{74}$ Folglich ergibt sich im Zuge der Erfolgsrechnung aus dem Saldo aus Erlösen und Kosten ein kalkulatorisches Betriebsergebnis, welches im Regelfall betragsmäßig ebenfalls vom GuV-Ergebnis abweicht. ${ }^{75}$ Zusätzlich zur Wirtschaftlichkeitskontrolle dient die Kosten- und Erlösrechnung, die das Kernstück des internen Rechnungswesens bildet, ${ }^{76}$ auch als Grundlage für die Angebotspreiskalkulation von Gütern und Dienstleistungen. ${ }^{77}$ Als zweites Rechenwerk befasst sich die Finanzplanung mit der Erfassung von Ein- und Auszahlungen. Das Ziel liegt hierbei in der laufenden Sicherstellung der kurz- und langfristigen Liquidität und somit des finanziellen Gleichgewichts des Unternehmens. ${ }^{78}$ Die Investitionsrechnung stellt - im Gegensatz zur Kosten- und Erlösrechnung sowie zur Finanzplanung - ein fallweise eingesetztes Instrument mit meist mehrperiodigem Zeithorizont dar. Zur Bestimmung der Vorteilhaftigkeit von Investitionen wird beispielsweise durch Diskontierung sämtlicher hiermit verbundener - d.h. gegenwärtiger und zukünftiger - Ein- und Auszahlungen der Kapitalwert dieser Projektvorhaben ermittelt. ${ }^{79}$ Grundsätzlich unterliegt die Gestaltung der Rechenwerke des internen Rechnungswesens nahezu keinerlei Restriktionen durch Unternehmensexterne und kann folglich den individuellen Bedürnissen

Vgl. Eisele (2002), S. 641f., Weißenberger (2003), S. 114f., Weber/Weißenberger (2006), S. 47f., Becker/Baltzer (2009a), S. 177 sowie Simons/Weißenberger (2009a), S. 393. Die Abgrenzung von pagatorischen Erträgen und kalkulatorischen Erlösen erfolgt grundsătzlich in analoger Weise. $\mathrm{Da}$ in der Unternehmenspraxis eine eigenständige Erlősrechnung jedoch - im Gegensatz zur Kostenrechnung - oftmals nicht vorhanden ist, wird in der Regel auf die Erträge der Finanzbuchhaltung zurilckgegriffen. Vgl. Becker/Baltzer (2009a), S. 177. Vgl. Coenenberg (1995), S. 2079, Weißenberger (1997), S. 29 sowie Becker/Baltzer (2009a), S. 177.

75 Vgl. Weißenberger (1997), S. 29, Kümpel (2002), S. 343 sowie Weißenberger (2003), S. 113.

76 Vgl. Eisele (2002), S. 635 sowie Weber/Weißenberger (2006), S. 355.

77 Vgl. Weißenberger (2003), S. 93f.

78 Vgl. Weißenberger (1997), S. 29, Weißenberger (2003), S. 95 sowie Weber/Kabst (2009), S. 186.

79 Vgl. auch zu den verschiedenen Verfahren der Investitionsrechnung Weißenberger (1997), S. 30, Weißenberger (2003), S. 95, Weber/Schäffer (2008), S. 322ff., Horváth (2009), S. 462 sowie Weber/Kabst (2009), S. 348ff. 
des Managements anpasst werden. ${ }^{80}$ Allerdings orientiert sich beispielsweise die Kosten- und Erlösrechnung in der Praxis meist an den allgemein üblichen Strukturen gängiger Kostenrechnungssysteme, wie z.B. der Vollkostenrechnung, der starren oder flexiblen Plankostenrechnung sowie der Deckungsbeitragsrechnung. ${ }^{81}$ Die wesentlichen Rechenzwecke des internen Rechnungswesens liegen in der Planung, Kontrolle und Dokumentation. ${ }^{82} \mathrm{Zu}$ Planungszwecken werden die Auswirkungen verschiedener Handlungsalternativen auf den finanziellen Zielerreichungsgrad des Unternehmens in Form von Prognoseinformationen aufgezeigt und somit die Grundlage für die Entscheidungsfindung gelegt. Des Weiteren werden aus diesen Prognoseinformationen Vorgabeinformationen abgeleitet, die den Entscheidungsträgern seitens der Unternehmensleitung als nicht zu über- bzw. unterschreitende Sollgrößen vorgegeben werden und deren Einhaltung im Zuge der Kontrolle überwacht wird. ${ }^{83}$ Die Dokumentation des Unternehmensprozesses durch das interne Rechnungswesen bildet die Basis für die übrigen beiden Rechnungszwecke und ist zudem von Bedeutung, wenn sich aus ihren Daten rechtliche oder vertragliche Pflichten ableiten. ${ }^{84}$

Die traditionelle Trennung in internes und externes Rechnungswesen im deutschsprachigen Raum geht auf die Denkansätze Schmalenbachs ${ }^{85}$ zurück. $^{86}$ Den Grund für

80 Vgl. Weißenberger (1997), S. 29, Kümpel (2002), S. 343 sowie Becker/Baltzer (2009a), S. 174. Eine Ausnahme ergibt sich in diesem Zusammenhang, wenn Unternehmen aufgrund von nicht zur Verfügung stehenden Marktpreisen zur Abrechnung offentlicher Auftrăge eine Preiskalkulation vornehmen. In diesem Fall müssen die vom Gesetzgeber formulierten Leitsătze für die Preisbildung aufgrund von Selbstkosten (LSP) beachtet werden, vgl. Weißenberger (1997), S. 29, Weißenberger (2003), S. 94, Weber/Weißenberger (2006), S. 358 sowie Günther/Zurwehme (2008), S. 102.

81 Vgl. Weißenberger (2003), S. 94. Zu den hier genannten Kostenrechnungssystemen vgl. exemplarisch die Ausführungen bei Weber/Weißenberger (2006).

82 Vgl. Pfaff(1994), S. 1068, Weber/Weißenberger (2006), S. 350 sowie Günther/Zurwehme (2008), S. 102. Teilweise wird in der Literatur auch Steuerung als Rechenzweck angefuhrt, die sich allerdings wiederum als Oberbegriff einer verbundenen zielorientierten Planung und Kontrolle verstehen lässt, vgl. Klein (1999), S. 13. Darüber hinaus wird statt von Steuerung auch vom Rechenzweck der Entscheidungsunterstultzung gesprochen, wobei hierunter auch die Verhaltenssteuerung - im Sinne einer Beeinflussung von Entscheidungen Dritter - subsumiert werden kann, vgl. Weißenberger (1997), S. 30.

Vgl. Pfaff (1994), S. 1068, Coenenberg (1995), S. 2078, Weißenberger (1997), S. 30, Weber/ Weißenberger (2006), S. 355 sowie Günther/Zurwehme (2008), S. 102. Zurwehme (2008), S. 102.

85 Schmalenbachs Hauptwerk „Dynamische Bilanz“ wurde erstmals 1919 veröffentlicht. Die letztmalig inhaltlich veränderte 13. Auflage erschien 1962, vgl. Schmalenbach (1962). 
diese Zweiteilung stellen dabei insbesondere die soeben aufgezeigten abweichenden Rechenzwecke zwischen dem internen Rechnungswesen einerseits und dem externen Rechnungswesen andererseits dar. ${ }^{87}$ In diesem Zusammenhang stellt Schneider (1997) fest:

„Der Rechnungszweck bestimmt über das Rechnungsziel den Rechnungsinhalt. ${ }^{\text {888 }}$

Während sich das externe Rechnungswesen auf sämtliche Aktivitäten des Unternehmens bezieht, ist insbesondere die interne Kosten- und Erlösrechnung auf das Ergebnis von Leistungserstellung und -verwertung gerichtet. ${ }^{89}$ Folglich erscheint im letzteren Falle der Einsatz von abweichenden Rechengrößen - d.h. kalkulatorischen Kosten bzw. Erlösen anstelle von pagatorischen Aufwendungen bzw. Erträgen - opportun, was auch plakativ als 'different costs for different purposes' bezeichnet werden kann. ${ }^{90}$ Die technisch-administrative Umsetzung dieses Nebeneinanders von interner Betriebsergebnisrechnung und externem Jahresabschluss wird dabei mithilfe von zwei separaten Rechenkreisen (Zweikreissystem) realisiert. ${ }^{91}$ Hieraus ergibt sich unter anderem der Vorteil, dass ein umfassender Abschluss aller Konten aufgrund des eigenständigen internen Rechenkreises nicht erforderlich ist und die - meist monatlich zu erstellende interne Betriebsergebnisrechnung folglich unabhängig von der externen Rechnungslegung durchgeführt werden kann.

Negative Konsequenzen bringt ein separiertes Rechnungswesen dagegen hinsichtlich der Funktion von Finanzinformationen des Rechnungswesens als Sprache bzw. Kommunikationsmittel mit sich. Da sich die Bezugsobjekte der internen Managementerfolgsrechnung (Produkte, Regionen, Geschäftsfelder, Projekte etc.) in der Regel von jenen des externen Rechnungswesens (Legaleinheiten) unterscheiden, fuhrt die Verwendung unterschiedlicher Wertansätze dazu, dass die Auswirkungen von Transaktionen auf die Bezugsobjekte des jeweils anderen Rechenkreises nicht mehr abschätzbar

86 Vgl. Ziegler (1994), S. 175f., Schildbach (1997), S. 262ff., Heyd (2001), S. 202 sowie Weißenberger (2003), S. 177ff.

87 Vgl. Ziegler (1994), S. 175f., Coenenberg (1995), S. 2078f., Haller (1997a), S. 115, Küting/ Lorson (1998), S. 486f. sowie Simons/Weißenberger (2009a), S. 393.

88 Schneider (1997), S. 45.

89 Vgl. auch im Folgenden Coenenberg (1995), S. 2078f.

90 Vgl. Klein (1999), S. 1 sowie Simons/Weißenberger (2009a), S. $390 \mathrm{f}$.

91 Vgl. auch im Folgenden Eisele (2002), S. 582ff. sowie Simons/Weißenberger (2009a), S. 393f. 
sind.$^{92}$ Folglich wird eine Übersetzung betriebswirtschaftlicher Maßnahmen und Ziele zwischen internem und externem Rechnungswesen aufgrund des fehlenden Zusammenhangs beider Rechenwerke nahezu unmöglich, wodurch es zu einem unüberbrückbaren Auseinanderfallen von interner und externer Finanzsprache kommt. Die durch diese Inkommensurabilitätsproblematik bedingte Verwirrung auf Seiten der jeweiligen Adressaten bzw. Nutzergruppen führt zu Dysfunktionalitäten, die bis hin zu ökonomischen Fehlentscheidungen reichen können. ${ }^{93}$

Im Gegensatz zum deutschsprachigen Raum existiert eine derartige Trennung der Erfolgsrechnungssysteme in angelsächsisch geprägten Ländern - etwa in den USA nicht. Da hier ausschließlich pagatorische Wertansätze für Zwecke der laufenden Ergebnisrechnung verwendet werden, liegt eine von Financial Accounting und Management Accounting gemeinsam genutzte Datenbasis vor (Einkreissystem). Diese Vorgehensweise liegt in der Zielsetzung der US-amerikanischen externen Rechnungslegung begründet, deren Hauptaufgabe es ist, entscheidungsrelevante Informationen für Investoren zu liefern. Durch diese Ausrichtung auf eine entscheidungsorientierte Informationsbereitstellung besteht eine weitgehende Übereinstimmung mit den Zielsetzungen des internen Rechnungswesens. Folglich wird den Rechnungslegungsdaten eine Eignung für sowohl unternehmensinterne als auch -externe Entscheidungssituationen zugesprochen. ${ }^{94}$

92 Vgl. auch für ein anschauliches Zahlenbeispiel bezüglich dieser Problematik Simons/Weißenberger (2009a), S. 394f.

Vgl. Belkaoui (1980), S. 362, Wagenhofer (2006), S. 13, Simons/Weißenberger (2008), S. 140, Simons/Weißenberger (2009a), S. 394f. sowie Simons/Weißenberger (2009b), S. 446. Die hier geschilderte Problematik führte im Lufthansa-Konzern 1991/92 zu einer schwerwiegenden Krise. Obwohl das interne, kalkulatorisch ermittelte Ergebnis einen Gewinn auswies, zeichnete sich von der Unternehmensleitung zunächst unbemerkt - in der externen Finanzberichterstattung ein Milliardenverlust und eine drohende Illiquidităt ab. Aufgrund dieses ,nahezu traumatischen Erlebnisses" setzte man sich bei Lufthansa fortan verstärkt mit der Integration des Rechnungswesens auseinander, vgl. Beißel/Steinke (2004), S. 63 sowie Kley (2006), S. 151f. (direktes Zitat auf S. 151).

94 Vgl. Haller (1997b), S. 272-274, Bruns (1999), S. 590f., Eisele (2002), S. 582f., Hebeler (2003), S. 134ff., Messner (2003), S. 25f. sowie Hoffjan (2008), S. 657. 


\section{Ansatzpunkte für eine Integration von internem und externem Rechnungswesen}

\subsection{Konzept eines integrierten Rechnungswesens}

Seit dem Verzicht auf eine eigenständige Kostenrechnung durch die Siemens AG ist die klassische Zweiteilung des betrieblichen Rechnungswesens zunehmend in Frage gestellt worden. ${ }^{95}$ Insbesondere aufgrund der mangelnden Kommunizierbarkeit von Abweichungen, die sich zwischen dem GuV-Ergebnis und dem intern ermittelten kalkulatorischen Betriebsergebnis ergeben, wurde in der Unternehmenspraxis fortan vermehrt dazu übergegangen, durch den Rückgriff auf eine einheitliche Datengrundlage für beide Rechenwerke das interne und externe Rechnungswesen $\mathrm{zu}$ integrieren. Wie Abbildung 5 zeigt, kommt es in einem solchen integrierten Rechnungswesen zu einer Verschiebung bei der Erfüllung der Rechenzwecke durch das interne bzw. externe Rechnungswesen.

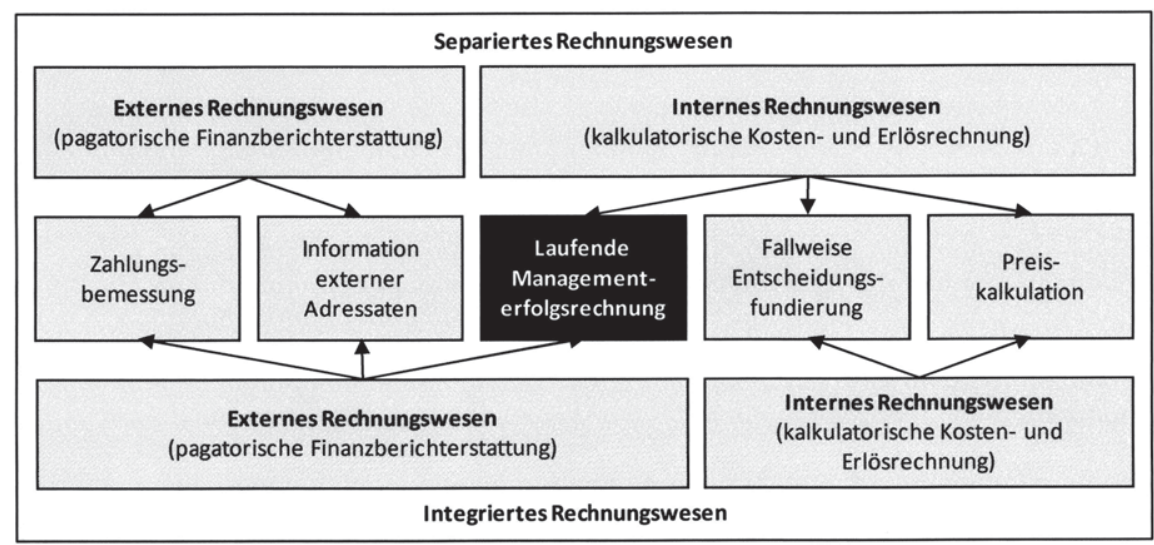

Abbildung 5: Gegenüberstellung von separiertem und integriertem Rechnungswesen"

So erfolgt die laufende Managementerfolgsrechnung, die innerhalb des separierten Rechnungswesens als kalkulatorische Betriebsergebnisrechnung vorgenommen wird, hier nunmehr auf Basis der pagatorischen Datenbasis der externen Finanzberichterstat-

95 Vgl. hierzu und im Folgenden die Ausführungen in Abschnitt Al der vorliegenden Arbeit sowie ergänzend Haller (1997b), S. 270f. und Weißenberger/Angelkort (2007a), S. 424.

96 Eigene Darstellung in Anlehnung an Weißenberger (2005), S. 192 sowie Weißenberger (2007a), S. 193. 
tung. Eine laufende Verbuchung von Zusatz- bzw. Anderskosten findet somit für Zwecke der internen Ergebnisrechnung nicht statt, da dies eine sehr komplexe und aufwändige Abstimmung zwischen internem und externem Ergebnis zur Folge hat. Für einzelfallbezogene Entscheidungsprobleme auf der operativen Produkt- und Prozessebene sowie Preiskalkulationen werden hingegen weiterhin kalkulatorische Wertansätze berücksichtigt. ${ }^{97}$

Durch die intern wie extern einheitlich verwendeten Bilanzierungs- und Bewertungsmethoden wird auf Unternehmens-, Segment- oder Geschäftsbereichsebene eine Ergebnisübereinstimmung erreicht, sodass die Entscheidungsunterstützung des internen Rechnungswesens auf Basis derselben Ergebnisgrößen erfolgt, die auch zu Informationszwecken für externe Adressaten veröffentlicht werden. Die Berichtsformate der internen Ergebnisrechnung - also beispielsweise die Strukturierung der Managementerfolgsrechnung als mehrstufige Deckungsbeitragsrechnung - bleiben von der Integration des Rechnungswesens jedoch ebenso unberührt wie die für Controllingzwecke weiterhin notwendigen Planungs-, Kalkulations- und Verrechnungsroutinen. ${ }^{98}$

Weitere Charakteristika eines integrierten Rechnungswesens liegen im organisatorischen sowie im systemtechnischen Bereich. Zur Erleichterung der vermehrt erforderlichen Zusammenarbeit zwischen den Akteuren des internen und externen Rechnungswesens ist die Schaffung formalisierter Kommunikationswege bzw. eine aufbauorganisatorische Zusammenfassung der Abteilungen Controlling und Bilanzierung in einem gemeinsamen Vorstandsressort förderlich. Darüber hinaus ist ein einheitlicher Kontenplan als Teil einer entsprechend integrierten IT-Systemlandschaft notwendig, um den Rückgriff auf eine gemeinsame Datenbasis im Zuge der internen und externen Erfolgsrechnung zu gewährleisten. ${ }^{99}$

Die steigende Kapitalmarktorientierung der Unternehmen sowie die zunehmende Internationalisierung der Rechnungslegungsnormen haben - dies wurde in Abschnitt Al der vorliegenden Arbeit gezeigt - zu einem wachsenden Interesse an der Thematik

Vgl. Heyd (2001), S. 208, International Group of Controlling (Hrsg.)/Weißenberger (2006), S. 45 sowie Weißenberger (2006b), S. 353f. 
der Integration des Rechnungswesens beigetragen. $\mathrm{Zu}$ begründen ist dieser Zusammenhang durch einen zentralen konzeptionellen Vorteil eines integrierten Rechnungswesens, von dem insbesondere kapitalmarktorientierte Unternehmen profitieren. Dieser wird darin gesehen, dass die Integration des Rechnungswesens durch den Rückgriff auf eine gemeinsame Datenbasis zur Schaffung einer einheitlichen Finanzsprache für interne und externe Kommunikationszwecke beiträgt, was auch als 'one version of the truth' bezeichnet werden kann. ${ }^{100}$ So können der im Falle eines separierten Rechnungswesens fehlende Zusammenhang von internen und externen Ergebnisgrößen sowie das hiermit einhergehende Auseinanderdriften von interner und externer Finanzsprache vermieden werden. Hierdurch wird die Gefahr von Missverständnissen und Fehlinterpretationen sowie von daraus ggf. resultierenden Führungsfehlern reduziert. Die Unternehmensleitung erhält folglich die Möglichkeit, die nach außen kommunizierten finanziellen Ziele intern durch Pläne auch auf nachgelagerte Steuerungsebenen herunterzubrechen und somit die Zielerreichung zu unterstützen. Gleichzeitig erlangt sie eine Sprachfähigkeit gegenüber dem Kapitalmarkt hinsichtlich der Erläuterung zurückliegender bzw. prognostizierter Entwicklungen der Geschäftssituation. ${ }^{101}$ Unterstützt wird diese Entwicklung zudem dadurch, dass die von kapitalmarktorientierten Konzernen verpflichtend anzuwendenden IFRS aufgrund ihrer informationsund entscheidungsorientierten Ausrichtung besser für eine Integration des internen und externen Rechnungswesens geeignet sind als die vom Gläubigerschutzgedanken geprägten Vorschriften des HGB.

Weitere wichtige in der Literatur ${ }^{102}$ genannte Vorteile der Integration liegen in einer schnelleren und weniger fehleranfälligen Berichterstattung sowie Kosteneinspa-

100 Vgl. Moussallem (2005), S. 11, Wagenhofer (2006), S. 13, Weißenberger (2007c), S. 328, Simons/Weißenberger (2009a), S. 391 sowie Simons/Weißenberger (2009b), S. 450. Im HenkelKonzern wird in diesem Zusammenhang vom so genannten 'one-number-principle' gesprochen, vgl. Hebeler (2006), S. 126 sowie Knobel/Vordermark (2008), S. 192.

Vgl. auch im Folgenden International Group of Controlling (Hrsg.)/Weißenberger (2006), S. 2528, Wagenhofer (2006), S. 13f., Weißenberger/Angelkort (2007a), S. 426, Weißenberger (2008a), Rz. 61-63 sowie Simons/Weißenberger (2009b), S. 446f. und 450. Jedoch ergeben sich aus der Integration des Rechnungswesens auch für nicht kapitalmarktorientierte Unternehmen - etwa aus dem Mittelstand - Kommunikationsvorteile, z.B. gegenüber Banken, Gesellschaftern sowie Geschăftspartnern wie Kunden und Lieferanten.

$102 \mathrm{Zu}$ den zentralen Vor- und Nachteilen einer Integration des Rechnungswesens aus Sicht der Unternehmenspraxis vgl. die Ausfuhrungen zu den diesbezulglich vorliegenden empirischen Ergebnissen in Abschnitt B4.2 der vorliegenden Arbeit. 
rungen aufgrund des Wegfallens von Doppelarbeiten und einer zentralen Datenhaltung bzw. -analyse. ${ }^{103}$

Neben diesen Vorteilen existiert jedoch auch eine Reihe von Kritikpunkten an der Integration des Rechnungswesens. So bedingt die Integration eine Abhängigkeit des Controllings von Änderungen der Bilanzierungs- und Bewertungsvorschriften des externen Rechnungswesens, da sich diese aufgrund der gemeinsam genutzten Datenbasis unmittelbar auf die interne Ergebnisrechnung auswirken. ${ }^{104}$ Neben den hiermit für die Anpassung der Controllingsysteme verbundenen Kosten kommt es zudem zu einer Beeinträchtigung von Zeitvergleichen. ${ }^{105}$ Zusätzlich zur reinen Änderungsdynamik ist darüber hinaus auch die inhaltliche Ausgestaltung der gesetzlichen Rechnungslegungsnormen aus Controllingperspektive mitunter problematisch. Zum einen sind die jeweiligen Standards häufig Resultat eines Kompromisses (z.B. die teilweise erfolgsneutrale Fair-Value-Bewertung) und keineswegs vollständig konsistent (z.B. die Diskontierung von Rückstellungen, nicht aber von latenten Steuern). ${ }^{106}$ Zum anderen dienen sie vorwiegend einem nicht an internen Erfordernissen ausgerichteten Zweck, wie etwa im Falle der IFRS der Informationsbereitstellung für Investoren. ${ }^{107}$ Folglich besteht ein weiterer konzeptioneller Nachteil der Integration des Rechnungswesens in der Gefahr, dass einzelne Rechnungslegungsnormen eine nicht controllinggerechte Wirkung mit sich bringen. ${ }^{108}$ Beispielsweise kann die Fair-Value-Bewertung dazu führen, dass sich in der internen Erfolgsrechnung zufallsbedingte Marktpreisschwankungen niederschlagen, die von der eigentlichen Managementleistung unabhängig und somit aus Controllingsicht unzweckmäßig sind.

gl. Erichsen (2000), S. 58, Jonen/Lingnau (2005), S. 286, Franz/Winkler (2006), S. 57, Wagenhofer (2006), S. 12f., Wala/Knoll/Messner (2007), S. 1882, Weißenberger (2007c), S. 328 sowie Weißenberger (2008a), Rz. 63.

I. Jonen/Lingnau (2005), S. 288, Weißenberger (2006b), S. 356 sowie Weißenberger (2008b), S. 442. Für den Fall eines IFRS-basierten integrierten Rechnungswesens wird der Controllerbereich diesbezüglich bei International Group of Controlling (Hrsg.)/Weißenberger (2006) plakativ als potenzielle „Geisel des IASB“ bezeichnet (S. 47).

Vgl. Simons/Weißenberger (2009b), S. 447.

Vgl. Wagenhofer (2006), S. 15.

Vgl. auch im Folgenden Wagenhofer (2006), S. 15f., Weißenberger (2006b), S. 354f. sowie Weißenberger (2008b), S. 440f.

Haeger (2006) stuft die zunehmende Fair-Value-Bewertung sogar als Gefahr einer „Divergenz von Rechnungswesen und Controlling“ ein (S. 265). Ähnlich kritisch bezüglich der Eignung der Fair-Value-Bewertung für Controllingzwecke äußert sich Ewert (2006), der ,aus einer Perspektive der anreizorientierten Performancesetzung den Anfang vom Ende der Ära einer Harmonisierung zwischen internem und externem Rechnungswesen heraufziehen" sieht (S. 203). 


\subsection{Muster einer partiellen Integration des Rechnungswesens}

Als Lösungsansatz hinsichtlich der soeben aufgezeigten Nachteile eines integrierten Rechnungswesens wird in der Literatur das Muster einer partiellen Integration des Rechnungswesens vorgeschlagen. ${ }^{109}$ Wie Abbildung 6 veranschaulicht, zeichnet sich dieses Integrationsmuster dadurch aus, dass die anvisierte Angleichung der internen und externen Ergebnisrechnung lediglich auf den obersten Hierarchieebenen - also neben der Gesamtunternehmens- bzw. Konzernebene und der darunter angesiedelten Segmentebene ggf. auch auf Geschäftsbereichs- und Profit Center-Ebene - erfolgt. Hingegen wird im Zuge der operativen Produkt- bzw. Prozesssteuerung weiterhin auf eigenständige kalkulatorische Größen zurückgegriffen. Diese können entsprechend des jeweiligen internen Verwendungszwecks - wie z.B. Kalkulation, Normalisierung oder Standardisierung - angepasst werden. Ein weiteres Charakteristikum der partiellen Integration liegt zudem darin, dass Abweichungen zwischen dem internen und dem externen Ergebnis grundsätzlich zulässig sind. Diese Ergebnisunterschiede ergeben sich aus einzelnen Überleitungs- bzw. Brückenpositionen, anhand derer die internen Steuerungskennzahlen um nicht controllinggerechte Effekte der externen Bilanzierungs- und Bewertungsvorschriften bereinigt werden. ${ }^{110}$

Vgl. auch im Folgenden Bruns (1999), S. 595f., Heyd (2001), S. 208, Jonen/Lingnau (2005), S. 288, Haller (2006), S. 155, International Group of Controlling (Hrsg.)/Weißenberger (2006), S. 50, Weißenberger (2007a), S. 198f., Weißenberger/Angelkort (2007a), S. 427f. sowie Simons/ Weißenberger (2009b), S. 447ff.

Die Deutsche Lufthansa AG eliminiert im Zuge der Überführung des Ergebnisses der betrieblichen Tătigkeit in das für interne Steuerungszwecke verwendete operative Ergebnis beispielsweise Aufwendungen und Ertrăge aus der Bildung von Drohverlustrückstellungen, Erträge aus der Auf1రsung von Rückstellungen, Gewinne und Verluste aus der Stichtagsbewertung von langfristigen Finanzschulden bzw. langfristigem Finanzvermögen sowie außerplanmäßige Ab- und Zuschreibungen auf Sachanlagen bzw. Goodwill, vgl. Beißel/Steinke (2004), S. 66f. sowie Beißel/Szczesny (2009), S. 26f. Im E.ON-Konzern wird das sowohl für die interne Steuerung als auch für die externe Kapitalmarktkommunikation eingesetzte 'Adjusted EBIT' ermittelt, indem das Ergebnis der gewőnnlichen Geschăftstätigkeit um das wirtschaftliche Zinsergebnis und das neutrale EBIT bereinigt wird. Als neutrale Ergebnisbestandteile werden dabei unter anderem Buchgewinne und -verluste aus dem Verkauf von Beteiligungen, Wertpapieren und ganzen Betriebsteilen einschließlich der damit verbundenen Transaktionskosten, Aufwendungen für Restrukturierungen und Kostenmanagement einschließlich der in diesem Zusammenhang zu bildenden Rückstellungen furr Sanierungen oder Stilllegungen sowie sonstige nicht operative Ergebnisse - wie z.B. auBerplanmäßige Abschreibungen oder unrealisierte Ergebnisse aus der stichtagsbezogenen Marktbewertung von Derivaten - angesehen, vgl. Haeger (2006), S. 248-250. Die Bertelsmann AG erachtet eine Anpassung der Daten des externen Rechnungswesens ebenfalls furr erforderlich, um dysfunktionale Effekte innerhalb der internen Steuerung - etwa aufgrund einer Fair ValueBewertung - zu vermeiden. Vgl. Erdmann (2008), S. 227. 


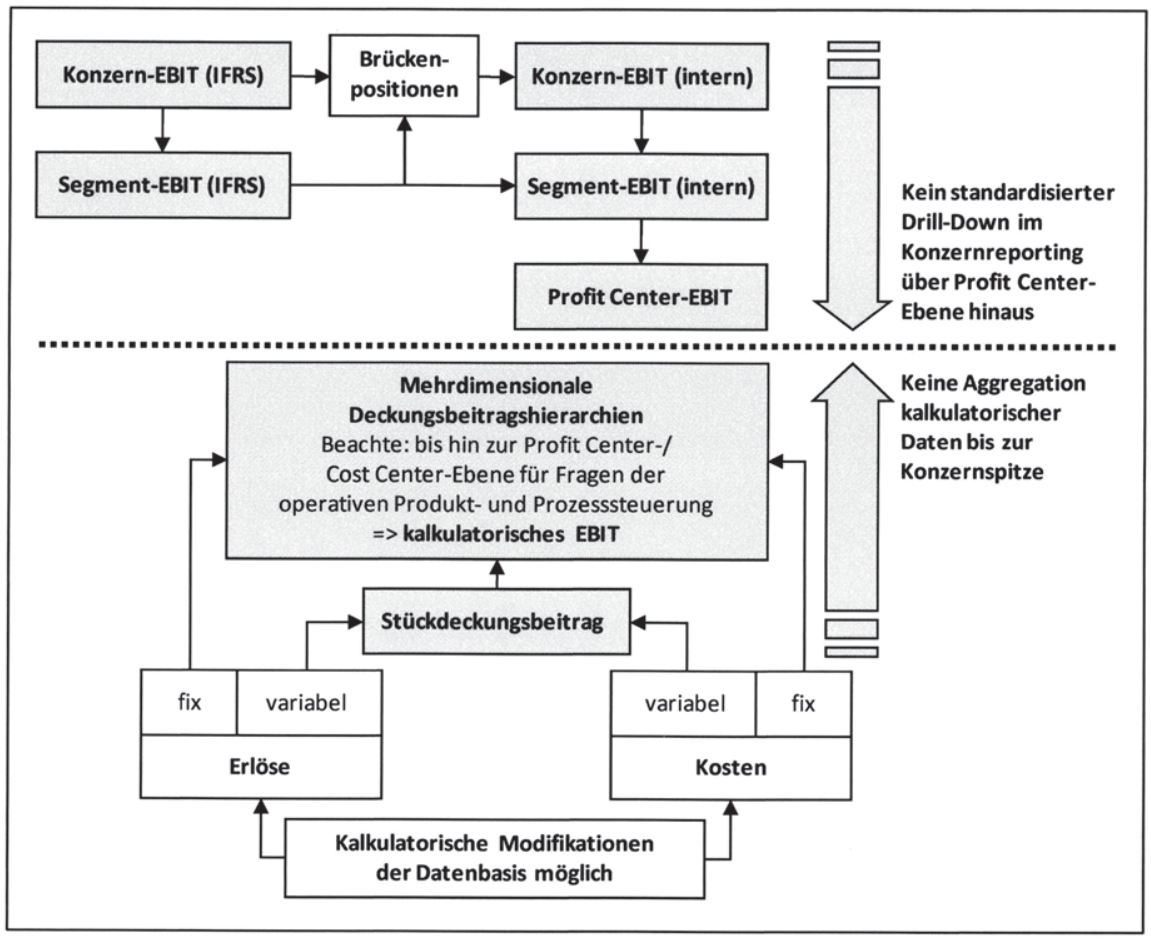

Abbildung 6: Partielle Integration des Rechnungswesens ${ }^{111}$

Die konzeptionelle Vorteilhaftigkeit des Musters einer partiellen Integration des Rechnungswesens besteht folglich darin, dass aufgrund des klaren Bezugs zwischen den internen und externen Ergebnisgrößen auf den oberen Hierarchieebenen eine Sprachfähigkeit gegenüber den Investoren geschaffen und die Entwicklung kapitalmarktorientierter Ziele und Maßnahmen ermöglicht wird. Die aus Controllingsicht wünschenswerte Flexibilität hinsichtlich der Anpassung bzw. Eliminierung einzelner Positionen der externen Rechnungslegung wird dabei weiterhin gewahrt. Die aus den vorgenommenen Änderungen resultierenden Ergebnisabweichungen können hierbei vorausgesetzt die Anzahl der Brückenpositionen ist überschaubar - im Rahmen einer Überleitungsrechnung nachvollziehbar plausibilisiert werden. Gleichzeitig kann auf der operativen Produkt- bzw. Prozessebene für Controllingzwecke weiterhin eine 
Ergebnisermittlung auf Basis kalkulatorischer Größen - etwa in Form einer Deckungsbeitragsrechnung - vorgenommen werden, ohne diese jedoch unternehmensweit $\mathrm{zu}$ aggregieren und auf Ebene der Konzernspitze für Steuerungszwecke heranzuziehen. ${ }^{112}$

Die Bruchstelle innerhalb der Ergebnisrechnung - angedeutet durch die gestrichelte Linie in Abbildung 6 - verlagert sich folglich. Statt wie bisher zwischen dem internen Ergebnis der Managementerfolgsrechnung und dem extern publizierten Ergebnis der Finanzberichterstattung verläuft sie im Rahmen eines partiell integrierten Rechnungswesens nun horizontal auf der Ebene der operativen Profit Center- bzw. Cost Center-Steuerung. Zwar ist ein Drill-Down der auf Konzernebene nach einheitlichen Bewertungsmaßstäben ermittelten und ggf. modifizierten Ergebnisgrößen bis hin auf die Produkt-, Prozess- oder Kostenstellenebene somit nicht mehr ohne weiteres möglich. Dies ist jedoch aus Controllingsicht unproblematisch, da die Steuerung im operativen Bereich oftmals sinnvoll auf Basis kalkulatorischer Standard- oder Opportunitätskosten bzw. anhand von leistungswirtschaftlichen Kennzahlen - wie z.B. Werttreiberhierarchien - erfolgt. Zudem stehen operative Entscheidungsträger meist ohnehin in keiner direkten Kommunikation mit Finanzinvestoren, sodass auf dieser Hierarchiestufe eine Überleitbarkeit zwischen internem und externem Ergebnis aufgrund der nicht bestehenden Auskunftspflicht nicht erforderlich ist. ${ }^{113}$

Zusammenfassend kann konstatiert werden, dass sich die Grundstruktur einer partiellen Integration des Rechnungswesens aus den jeweils unterschiedlichen Informationsbedarfen auf den verschiedenen Unternehmenshierarchiestufen bzw. Leitungsebenen ergibt. Dieses Muster lässt sich durch den folgenden Grundsatz charakterisieren:

„Je operativer das interne Rechnungswesen [...], desto geringer die Möglichkeiten einer Integration. “114

Vgl. Bruns (1999), S. 595f., Heyd (2001), S. 208, Jonen/Lingnau (2005), S. 288, International Group of Controlling (Hrsg.)/Weißenberger (2006), S. 51, Weißenberger (2007a), S. 200f., Weißenberger/Angelkort (2007a), S. 429 sowie Simons/Weißenberger (2009b), S. 448ff. Group of Controlling (Hrsg.)/Weißenberger (2006), S. 51f., Weißenberger (2007a), S. 201-203, Weißenberger/Angelkort (2007a), S. 429-431 sowie Simons/Weißenberger (2009b), S. 448ff. Für ein Praxisbeispiel hinsichtlich des Einsatzes von Werttreiberbetrachtungen vgl. Kümmel/Watterott (2005), S. 14-18. 
So benötigt das Management auf den oberen Leitungsebenen für Steuerungszwecke in erster Linie pagatorische Finanz-, Bilanz- sowie Aufwands- und Ertragskennzahlen, die in einem klaren Zusammenhang mit den im Rahmen der externen Finanzberichterstattung gegenüber dem Kapitalmarkt kommunizierten Größen stehen. Im operativ geprägten Bereich hingegen sind verstärkt kalkulatorische bzw. leistungswirtschaftliche Kennzahlen zur - oftmals einzelfallbezogenen - Entscheidungsfundierung erforderlich. $^{115}$

\section{Stand der empirischen Forschung zur Integration des Rech- nungswesens}

\section{1 Überblick über bisherige Studien}

Wie bereits im Rahmen der Einleitung der vorliegenden Arbeit aufgezeigt wurde, sind seit Beginn der wissenschaftlichen Diskussion im Jahr 1994 zahlreiche empirisch ausgerichtete Literaturbeiträge zum Themenfeld der Integration des internen und externen Rechnungswesens verfasst worden. Trotz dieser Vielzahl an Publikationen befinden sich jedoch nur wenige großzahlige Erhebungen darunter, die sich schwerpunktmäßig mit der Integration des Rechnungswesens im deutschsprachigen Raum beschäftigen. In Tabelle 2 werden diese überwiegend erst in jüngster Zeit veröffentlichten empirischen Studien überblicksartig vorgestellt. ${ }^{116}$

Wie die hier aufgeführten Informationen erkennen lassen, überwiegt die Erhebungsmethode der schriftlichen Befragung mittels standardisiertem Fragebogen. Ebenfalls eine Rolle spielen zudem die Analyse von Geschäftsberichten oder sonstigen Dokumenten sowie Experteninterviews.

115 Vgl. Bruns (1999), S. 595f., Heyd (2001), S. 208, Schweitzer (2003), S. 440ff., Beißel/Steinke (2004), S. 62, Jonen/Lingnau (2005), S. 288, International Group of Controlling (Hrsg.)/Weißenberger (2006), S. 51f., Weißenberger (2007a), S. 200-203, Weißenberger/Angelkort (2007a), S. 429-431 sowie Simons/Weißenberger (2009b), S. 448ff.

Neben den neun in Abschnitt Al der vorliegenden Arbeit angesprochenen Studien, die allesamt bis 2007 veröffentlicht wurden, zählen hierzu vier weitere seitdem erschienene Untersuchungen (die Studie von Angelkort/Sandt/Weißenberger (2008b) ist eine Erweiterung der Studie von Weißenberger/Angelkort (2007b); folglich werden diese beiden zeitversetzt publizierten Untersuchungen als zusammengehörig behandelt). 


\begin{tabular}{|c|c|c|c|c|c|c|}
\hline$\frac{0}{\overline{0}}$ & Studie & Thema & $\begin{array}{l}\text { Untersuchungsobjekte/ } \\
\text { Stichprobenumfang }\end{array}$ & $\begin{array}{l}\text { Erhebungs- } \\
\text { methodik }\end{array}$ & $\begin{array}{l}\text { Analyse- } \\
\text { verfahren }\end{array}$ & $\begin{array}{l}\text { Untersu- } \\
\text { chungstyp }\end{array}$ \\
\hline 줄. & $\begin{array}{l}\text { Horváth/Arnaout } \\
\text { (1997) }\end{array}$ & $\begin{array}{l}\text { State of the Art und Implemen- } \\
\text { tierungsstand internationaler } \\
\text { Rechnungslegungsvorschriften } \\
\text { und der Integration des Rech- } \\
\text { nungswesens in Deutschland }\end{array}$ & $\begin{array}{l}56 \text { der } 200 \text { umsatzstärksten } \\
\text { Unternehmen Deutschlands }\end{array}$ & $\begin{array}{l}\text { Schriftliche } \\
\text { Befragung mit } \\
\text { standardisiertem } \\
\text { Fragebogen }\end{array}$ & $\begin{array}{l}\text { Deskriptive } \\
\text { Statistik }\end{array}$ & $\begin{array}{l}\text { Deskriptiv- } \\
\text { explorativ }\end{array}$ \\
\hline 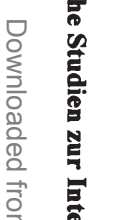 & Hoke (2001) & $\begin{array}{l}\text { Konzernsteuerung auf Basis eines } \\
\text { integrierten Rechnungswesens im } \\
\text { Kontext internationaler Rech- } \\
\text { nungslegungsstandards }\end{array}$ & $\begin{array}{l}41 \text { von } 86 \text { Unternehmen des } \\
\text { DAX } 100 \text { (Finanzdienstleister } \\
\text { wurden ausgeschlossen) }\end{array}$ & $\begin{array}{l}\text { - Analyse der } \\
\text { Geschäfts- } \\
\text { berichte } \\
\text { - Experten- } \\
\text { interviews }\end{array}$ & $\begin{array}{l}\text { - Qualitative } \\
\text { Analyse } \\
\text { - Deskriptive } \\
\text { Statistik } \\
\text { - Kreuztabel- } \\
\text { ierung }\end{array}$ & $\begin{array}{l}\text { Deskriptiv- } \\
\text { explorativ }\end{array}$ \\
\hline 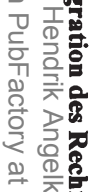 & Melcher (2002) & $\begin{array}{l}\text { Analyse der Überleitungsrech- } \\
\text { nungen zwischen Erfolgskenn- } \\
\text { zahlen des internen und externen } \\
\text { Rechnungswesens am Beispiel } \\
\text { der Segmentberichterstattung }\end{array}$ & $\begin{array}{l}24 \text { Unternehmen des DAX } 30 \\
\text { (Finanzdienstleister wurden } \\
\text { ausgeschlossen) }\end{array}$ & $\begin{array}{l}\text { Analyse der } \\
\text { Geschäfts- } \\
\text { berichte }\end{array}$ & $\begin{array}{l}\text { Qualitative } \\
\text { Analyse }\end{array}$ & $\begin{array}{l}\text { Deskriptiv- } \\
\text { explorativ }\end{array}$ \\
\hline 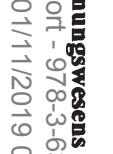 & $\begin{array}{l}\text { Haring/Prantner } \\
(2005)\end{array}$ & $\begin{array}{l}\text { Stand der Integration des } \\
\text { Rechnungswesens im } \\
\text { deutschsprachigen Raum }\end{array}$ & $\begin{array}{l}83 \text { der } 870 \text { umsatzstärksten } \\
\text { Unternehmen Deutschlands und } \\
\text { Österreichs (Finanzdienstleister } \\
\text { wurden ausgeschlossen) }\end{array}$ & $\begin{array}{l}\text { Schriftliche } \\
\text { Befragung mit } \\
\text { standardisiertem } \\
\text { Fragebogen }\end{array}$ & $\begin{array}{l}\text { Deskriptive } \\
\text { Statistik }\end{array}$ & $\begin{array}{l}\text { Deskriptiv- } \\
\text { explorativ }\end{array}$ \\
\hline
\end{tabular}




\begin{tabular}{|c|c|c|c|c|c|c|}
\hline 军 & Studie & Thema & $\begin{array}{l}\text { Untersuchungsobjekte/ } \\
\text { Stichprobenumfang }\end{array}$ & $\begin{array}{l}\text { Erhebungs- } \\
\text { methodik }\end{array}$ & $\begin{array}{l}\text { Analyse- } \\
\text { verfahren }\end{array}$ & $\begin{array}{l}\text { Untersu- } \\
\text { chungstyp }\end{array}$ \\
\hline 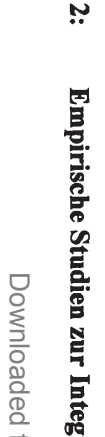 & Müller (2006) & $\begin{array}{l}\text { Stand der Integration des } \\
\text { Rechnungswesens unter } \\
\text { besonderer Berücksichtigung der } \\
\text { Prinzipal-Agenten-Theorie }\end{array}$ & $\begin{array}{l}81 \text { von } 321 \text { Unternehmen des } \\
\text { Prime Standard der Deutschen } \\
\text { Börse }\end{array}$ & $\begin{array}{l}\text { Schriftliche } \\
\text { Befragung mit } \\
\text { standardisiertem } \\
\text { Fragebogen }\end{array}$ & $\begin{array}{l}\text { - Deskriptive } \\
\text { Statistik } \\
\text { - Mittelwert- } \\
\text { vergleiche } \\
\text { - Korrelations- } \\
\text { analyse } \\
\text { - Kreuztabel- } \\
\text { lierung } \\
\text { - Two-Step- } \\
\text { Clusteranalyse } \\
\text { - Regressions- } \\
\text { analyse }\end{array}$ & $\begin{array}{r}\text { - Deskriptiv- } \\
\text { explorativ } \\
\text { - Explanativ }\end{array}$ \\
\hline 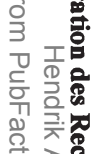 & $\begin{array}{l}\text { Wagenhofer/ } \\
\text { Engelbrechts- } \\
\text { müller (2006) }\end{array}$ & $\begin{array}{l}\text { Controlling und Reporting vor } \\
\text { dem Hintergrund der Anfor- } \\
\text { derungen internationaler } \\
\text { Rechnungslegungsstandards }\end{array}$ & $\begin{array}{l}97 \text { der } 508 \text { größten bzw. im } \\
\text { Prime Market der Wiener Börse } \\
\text { notierten Unternehmen Öster- } \\
\text { reichs }\end{array}$ & $\begin{array}{l}\text { Schriftliche } \\
\text { Befragung mit } \\
\text { standardisiertem } \\
\text { Fragebogen }\end{array}$ & $\begin{array}{l}\text { Deskriptive } \\
\text { Statistik }\end{array}$ & $\begin{array}{l}\text { Deskriptiv- } \\
\text { explorativ }\end{array}$ \\
\hline 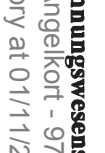 & $\begin{array}{l}\text { Grieshop/Weber } \\
\text { (2007) }\end{array}$ & $\begin{array}{l}\text { Kooperation von Controller- } \\
\text { bereich und externem Rech- } \\
\text { nungswesen }\end{array}$ & $\begin{array}{l}1.057 \text { von } 4.304 \text { Unternehmen } \\
\text { unterschiedlicher Größenklassen } \\
\text { und Branchen }\end{array}$ & $\begin{array}{l}\text { Schriftliche } \\
\text { Befragung mit } \\
\text { standardisiertem } \\
\text { Fragebogen }\end{array}$ & $\begin{array}{l}\text { Kovarianzstruk- } \\
\text { turanalyse }\end{array}$ & Explanativ \\
\hline
\end{tabular}




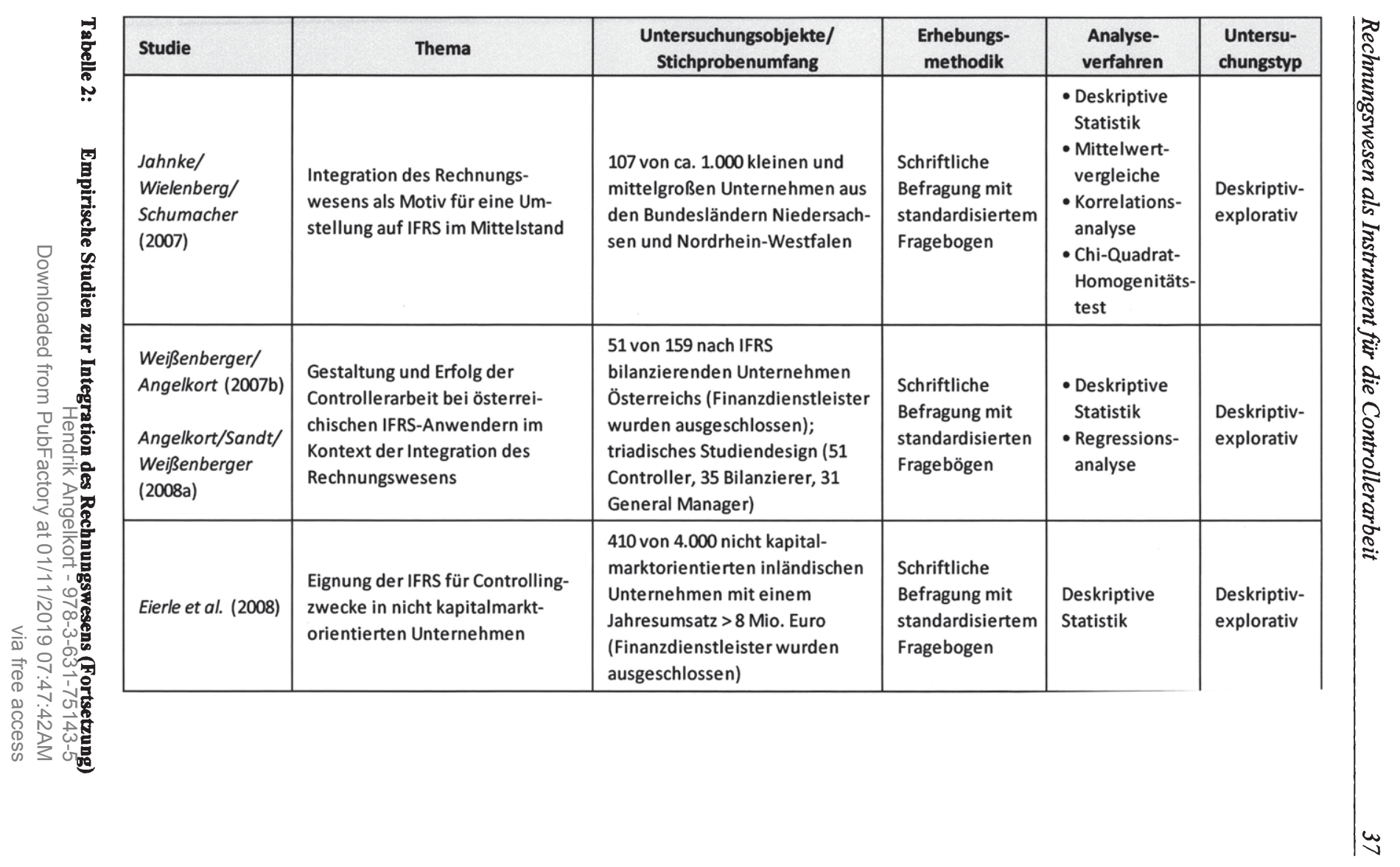




\begin{tabular}{|c|c|c|c|c|c|c|}
\hline 兽 & Studie & Thema & $\begin{array}{l}\text { Untersuchungsobjekte/ } \\
\text { Stichprobenumfang }\end{array}$ & $\begin{array}{l}\text { Erhebungs- } \\
\text { methodik }\end{array}$ & $\begin{array}{l}\text { Analyse- } \\
\text { verfahren }\end{array}$ & $\begin{array}{l}\text { Untersu- } \\
\text { chungstyp }\end{array}$ \\
\hline 통 & $\begin{array}{l}\text { Blase/Müller } \\
\text { (2009) }\end{array}$ & $\begin{array}{l}\text { Integration von interner und } \\
\text { externer Segmentbericht- } \\
\text { erstattung im Rahmen der vor- } \\
\text { zeitigen Umstellung auf IFRS } 8\end{array}$ & $\begin{array}{l}\text { 13 IFRS-8-Erstanwender von } 115 \\
\text { Unternehmen des DAX, MDAX } \\
\text { und SDAX (Finanzdienstleister } \\
\text { wurden ausgeschlossen) }\end{array}$ & $\begin{array}{l}\text { Analyse der } \\
\text { Geschäfts- } \\
\text { berichte }\end{array}$ & $\begin{array}{l}\text { - Qualitative } \\
\text { Analyse } \\
\text { - Deskriptive } \\
\text { Statistik }\end{array}$ & $\begin{array}{l}\text { Deskriptiv- } \\
\text { explorativ }\end{array}$ \\
\hline 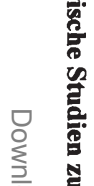 & Bramann (2009) & $\begin{array}{l}\text { Auswirkungen der Harmonisie- } \\
\text { rung internationaler Rechnungs- } \\
\text { legung auf das F\&E-Controlling }\end{array}$ & $\begin{array}{l}\text { Zehn börsennotierte, nach IFRS } \\
\text { bilanzierende Unternehmen aus } \\
\text { F\&E-intensiven Branchen (16 } \\
\text { Unternehmensvertreter, vier } \\
\text { Wirtschaftsprüfer) }\end{array}$ & $\begin{array}{l}\text { - Dokumenten- } \\
\text { analyse } \\
\text { - Experten- } \\
\text { interviews }\end{array}$ & $\begin{array}{l}\text { - Qualitative } \\
\text { Analyse } \\
\text { - Deskriptive } \\
\text { Statistik }\end{array}$ & $\begin{array}{l}\text { Deskriptiv- } \\
\text { explorativ }\end{array}$ \\
\hline 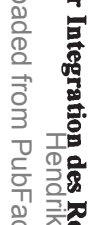 & Weide (2009) & $\begin{array}{l}\text { Gestaltung und Erfolg des } \\
\text { Management Reporting im } \\
\text { Kontext der Integration des } \\
\text { Rechnungswesens }\end{array}$ & $\begin{array}{l}28 \text { von } 54 \text { kapitalmarktorientier- } \\
\text { ten, nach IFRS bilanzierenden } \\
\text { Großunternehmen im deutsch- } \\
\text { sprachigen Raum ( } 34 \text { Unter- } \\
\text { nehmensvertreter; Finanzdienst- } \\
\text { leister wurden ausgeschlossen) }\end{array}$ & $\begin{array}{l}\text { - Dokumenten- } \\
\text { analyse } \\
\text { - Experten- } \\
\text { interviews }\end{array}$ & $\begin{array}{l}\text { - Qualitative } \\
\text { Analyse } \\
\text { - Deskriptive } \\
\text { Statistik } \\
\text { - Inhaltsanalyse }\end{array}$ & $\begin{array}{l}\text { Deskriptiv- } \\
\text { explorativ }\end{array}$ \\
\hline
\end{tabular}


Bei der Auswahl der Untersuchungsobjekte zeigt sich, dass Finanzdienstleister, wie z.B. Banken oder Versicherungen, aufgrund der branchenspezifischen Besonderheiten sowie der speziellen für diese Unternehmen geltenden Rechnungslegungsvorschriften in der Mehrheit der Studien explizit nicht berücksichtigt wurden. ${ }^{117}$ Abgesehen von dieser Gemeinsamkeit variiert die Auswahl der zu untersuchenden Unternehmen allerdings recht deutlich. So wird teilweise ausschließlich auf börsennotierte Unternehmen, auf IFRS-Anwender oder auf mittelständische bzw. nicht kapitalmarktorientierte Unternehmen fokussiert, wodurch die Generalisierbarkeit bzw. Vergleichbarkeit der einzelnen Studienergebnisse erheblich eingeschränkt wird. ${ }^{118}$ Hinzu kommt, dass auch der Stichprobenumfang mit absoluten Fallzahlen zwischen zehn und 1.057 Fällen sehr heterogen ausfällt.

Des Weiteren kann konstatiert werden, dass bisher überwiegend rein deskriptivexplorativ konzipierte Untersuchungen durchgeführt wurden, bei denen zudem keine fortgeschrittenen Analysemethoden der empirischen Sozialforschung zum Einsatz kamen. Die beiden diesbezüglich einzigen Ausnahmen bilden die Studie von Müller (2006), die neben deskriptiv-explorativen auch explanative, d.h. hypothesenbasierte Elemente beinhaltet, sowie die Studie von Grieshop/Weber (2007), die mit der Kovarianzstrukturanalyse ein leistungsstarkes und explanativ ausgerichtetes Analyseverfahren verwendet. Dies deutet darauf hin, dass das Forschungsgebiet der Integration des internen und externen Rechnungswesens durch einen bislang noch eingeschränkten Erkenntnisstand gekennzeichnet ist. ${ }^{119}$

\subsection{Wesentliche Ergebnisse}

Im nächsten Schritt werden die wichtigsten Kernergebnisse der im vorangegangenen Abschnitt aufgeführten empirischen Untersuchungen zur Integration des Rechnungswesens vorgestellt. Dabei wird der Fokus auf zentrale Themencluster gelegt, die im Zusammenhang mit den Forschungsfragen der vorliegenden Arbeit stehen.

117 Vgl. für diese Begründung exemplarisch Hoke (2001), S. 147.

118 Verstărkt wird diese Problematik dadurch, dass in den Studien von Haring/Prantner (2005) und Weißenberger/Angelkort (2007b) bzw. Angelkort/Sandt/Weißenberger (2008a) teilweise bzw. ausschließlich osterreichische Unternehmen befragt wurden, wohingegen alle übrigen Studien auf Unternehmen in Deutschland abzielen. 
Stand der Integration des internen und externen Rechnungswesens

Der empirischen Erfassung des Stands der Integration des Rechnungswesens kommt eine hohe Bedeutung $\mathrm{zu}$, da sie nicht nur in isolierter Form von Interesse ist, sondern zugleich den Ausgangspunkt für weiterführende integrationsabhängige Analysen bildet. Die bislang existierenden Studien deuten dabei auf ein über die Zeit tendenziell gestiegenes Ausmaß der Integration hin. ${ }^{120}$ So gaben in der Studie von Horváth/ Arnaout (1997) lediglich 22\% der Unternehmen an, die Integration des Rechnungswesens bereits abgeschlossen zu haben, ${ }^{121}$ während sich bei Hoke (2001) bereits $36 \%$ der Teilnehmer entsprechend äußerten. ${ }^{122}$ Im Rahmen der Untersuchung von Haring/ Prantner (2005) stieg der Anteil der Unternehmen mit - nach eigener Einschätzung integriertem Rechnungswesen auf $40 \%$ (48\% in Deutschland, $34 \%$ in Österreich). ${ }^{123}$ In der Erhebung von Wagenhofer/Engelbrechtsmüller (2006) bescheinigten sich $75 \%$ der HGB-Anwender und $86 \%$ der nach internationalen Rechnungslegungsstandards bilanzierenden Unternehmen eine abgeschlossene Integration des Rechnungswesens. ${ }^{124}$ In der Studie von Weißenberger/Angelkort (2007b) war dies bei $76 \%$ der befragten Controller der Fall. ${ }^{125}$

Hinsichtlich der hier skizzierten Ergebnisse ist jedoch zu bemängeln, dass der Stand der Integration lediglich direkt erfragt wurde. Eine Operationalisierung - etwa durch Abfragen unterschiedlicher Facetten der Vereinheitlichung des internen und externen Rechnungswesens - wurde nicht vorgenommen, sodass ein einheitliches Verständnis des Integrationsbegriffs bei den Probanden nicht sichergestellt ist. ${ }^{126}$

Eine diesbezüglich geeignetere Vorgehensweise liegt den Studien von Müller (2006) und Weide (2009) zu Grunde. So ermittelt Müller (2006) für jedes Unternehmen einen Harmonisierungsgrad auf Basis eines drei verschiedene Dimensionen

An dieser Stelle sei jedoch erneut auf die nur eingeschränkte Vergleichbarkeit der verschiedenen Studien hingewiesen.

Vgl. Horváth/Arnaout (1997), S. 265.

Vgl. Hoke (2001), S. 161.

Vgl. Haring/Prantner (2005), S. 151.

Vgl. Wagenhofer/Engelbrechtsmüller (2006), S. 22.

Vgl. Weißenberger/Angelkort (2007b), S. 28.

Für eine ahnliche Kritik vgl. Weide (2009), S. 220. Für empirische Hinweise auf ein in der Unternehmenspraxis vorliegendes uneinheitliches Verständnis bezüglich der Integration des Rechnungswesens vgl. Hoke (2001), S. 153. 
umfassenden Scoring-Modells. ${ }^{127}$ Insgesamt erzielen $68 \%$ der Unternehmen einen Harmonisierungsgrad, der in den Bereich zwischen 'Harmonisierung überwiegt Divergenz' und 'Weitgehende Harmonisierung' fällt. ${ }^{128}$ Bei Weide (2009) wird der Integrationsstand anhand der Kriterien eines einheitlichen Periodenergebnisses, eines einheitlichen Kontenplans und einheitlicher Bilanzierungs- und Bewertungsregeln ermittelt. $62,5 \%$ der Unternehmen erfüllen alle drei der herangezogenen Integrationskriterien, während sämtliche Unternehmen mindestens eines dieser Kriterien erfüllen. ${ }^{129}$

Beide Ansätze bieten somit zwar eine differenziertere Erfassung des Stands der Integration des Rechnungswesens als die zuvor genannten Studien, sind aber dennoch nicht detailliert bzw. umfassend genug, da sie z.B. keine ablauf- bzw. aufbauorganisationsbezogenen Aspekte berücksichtigen. Die Studie von Müller (2006) basiert zudem ausschließlich auf börsennotierten Unternehmen. Dies hat zur Folge, dass der Einfluss eben dieser Börsennotierung - als Beispiel für einen wichtigen Kontextfaktor - auf das Ausmaß der Integration des Rechnungswesens zwangsläufig nicht Bestandteil der Analyse ist. Darüber hinaus ist der ermittelte Integrationsstand zeitlich überholt - diese Problematik ergibt sich im Zeitablauf naturgemäß für sämtliche auf einen Status quo bezogene Untersuchungen -, da die Datenerhebung bereits im Jahr 2004 erfolgte. ${ }^{130}$ Der Bestandsaufnahme von Weide (2009) liegt dagegen - bedingt durch die gewählte Erhebungsmethodik - eine nur als gering einzustufende absolute Stichprobengröße zu Grunde, wodurch die Generalisierbarkeit ihrer Ergebnisse eingeschränkt wird.

\section{Umsetzung des Musters einer partiellen Integration des Rechnungswesens}

Neben der Erfassung des Integrationsstands auf Gesamtunternehmensebene ist für eine umfassende Ermittlung des Status quo auch eine hierarchiestufenabhängige Betrachtung erforderlich. Dennoch liegen bisher zum Stand der Umsetzung einer partiellen tion direkt erfragt. 66\% (53 von 80) der Unternehmen gaben hierbei an, die Integration des Rechnungswesens bereits umgesetzt zu haben, vgl. Müller (2006), S. 189. 
Integration des Rechnungswesens nur vergleichsweise wenige empirische Ergebnisse vor. ${ }^{131}$ Erste qualitative Hinweise liefert hier die Untersuchung von Hoke (2001):

„Die Befragung der Unternehmen ergab, dass eine Abweichung von der Wertbasis der externen Rechnungslegung eher auf nachgeordneten Unternehmensebenen praktiziert bzw. dort für sinnvoll gehalten wird. So werden in Unternehmen, die auf Konzernebene auf den Einsatz kalkulatorischer Kosten verzichten und dort eine Vereinheitlichung durchgefüht haben, auf operativer Steuerungsebene kalkulatorische Kosten im Rahmen des internen Berichtswesens durchaus noch verwendet. " "l32

In der Studie von Müller (2006) zeigt sich, dass in 41 der teilnehmenden Unternehmen auf Konzern- bzw. Unternehmensleitungsebene Kennzahlen auf Basis pagatorischer Wertansätze ('Aufwandsgrößen') zu Steuerungszwecken eingesetzt werden, während dies auf Geschäftsbereichs-/Divisionsebene lediglich bei 34 und auf Produkt- und Prozessebene bei 23 Unternehmen der Fall ist. Hingegen gewinnt der Einsatz kalkulatorischer Steuerungsgrößen ('Kostengrößen') auf nachgelagerten Hierarchieebenen an Bedeutung. So steigt deren Verwendung von 21 Nennungen auf Ebene der Konzernbzw. Unternehmensleitung über 24 Fälle im Rahmen der Geschäftsbereichs-/ Divisionsleitung auf 30 Unternehmen im Bereich der Produkt- und Prozesssteuerung. ${ }^{133}$

In der Untersuchung von Weißenberger/Angelkort (2007b) geben zudem $88 \%$ der teilnehmenden österreichischen IFRS-Anwender an, auf Gesamtunternehmensebene ihre Ergebnisplanung auf IFRS-Basis durchzufuihren, wohingegen sich für die Ge-

131 Simons/Weißenberger (2009b) weisen in diesem Zusammenhang darauf hin, dass in den fruhen Veröffentlichungen zur Integration des Rechnungswesens Abweichungen in der Integrationsintensităt auf unterschiedlichen Hierarchiestufen nicht thematisiert wurden, vgl. Simons/ Weißenberger (2009b), S. 449. Dementsprechend war die theoretisch-konzeptionelle Grundlage für diesbezligliche empirische Analysen zunächst nicht gegeben. Hoke (2001), S. 186.

133 Vgl. Müller (2006), S. 216. Ähnliche Anhaltspunkte für eine partielle Integration liefern die Studienergebnisse von Wagenhofer/Engelbrechtsmüller (2006), S. 25. Wăhrend hier auf Ebene des Top-Managements in erster Linie finanzielle und bilanzielle Daten sowie renditebasierte Kennzahlen zu den am hăufigsten genannten Leistungsindikatoren zählen, stehen auf den nachgelagerten Hierarchiestufen verstärkt kostenrechnerische Daten im Vordergrund. 
schäftsbereichsebene nur noch 57\% und die Produktebene lediglich $33 \%$ der Unternehmen entsprechend äußern. ${ }^{134}$

Im Rahmen der von Weide (2009) durchgeführten Experteninterviews stimmen 22 von 24 befragten Unternehmen des Hauptsamples der Aussage zu, dass vor allem die Ergebnisrechnung auf Konzernebene den relevanten Integrationsbereich bilden sollte. Während sich des Weiteren 17 Unternehmen dahingehend äußern, dass für die Fundierung operativer Produkt- und Prozessentscheidungen nicht auf die Wertansätze der externen IFRS-Finanzberichterstattung zurückgegriffen wird, schließen drei Unternehmen auch diese Ebene explizit in den relevanten Integrationsbereich mit ein. ${ }^{135}$

Die hier skizzierten Ergebnisse liefern zwar erste Anhaltspunkte hinsichtlich der Umsetzung des Musters einer partiellen Integration des Rechnungswesens. Diese sind allerdings noch recht grob, da sie aufgrund der ihnen zu Grunde liegenden Häufigkeitsbetrachtungen nur Aussagen für die Gesamtheit der jeweils untersuchten Unternehmen ermöglichen. Eine individuelle Erfassung des Integrationsmusters jedes einzelnen teilnehmenden Unternehmens und eine darauf aufbauende Quantifizierung von auftretenden Integrationsmustern wurden dagegen noch nicht durchgeführt.

Vor- und Nachteile der Integration des Rechnungswesens

Die Fragestellung bezüglich der mit einer Integration des internen und externen Rechnungswesens assoziierten Vor- und Nachteile wurde seit Beginn der Integrationsdiskussion bereits mehrfach - konkret durch Horváth/Arnaout (1997), Hoke (2001), Haring/Prantner (2005), Müller (2006) und Weißenberger/Angelkort (2007b) - empirisch untersucht. Als gemeinsames Kernergebnis lässt sich festhalten, dass die zentralen Vorteile der Integration in

- der Reduktion von Kommunikationsschwierigkeiten,

- der Erhöhung der (internationalen) Verständlichkeit und Transparenz des Rechnungswesens sowie

- der Schaffung einer gemeinsamen Datenbasis und der Verwendung einheitlicher Kennzahlen 
gesehen werden. ${ }^{136}$ Damit bewegen sich die maßgeblichen Vorteile überwiegend auf der Kommunikationsebene. Als bedeutsame Nachteile der Integration nennen die befragten Unternehmensvertreter mehrheitlich

- die Abhängigkeit von externen Normensetzungsprozessen sowie

- die schlechtere Erfüllung der Controllinganforderungen. ${ }^{137}$

Diesbezüglich ist jedoch anzumerken, dass in den Untersuchungen von Horváth/ Arnaout (1997) und Haring/Prantner (2005) sogar 29\% bzw. 47\% der Unternehmen explizit angeben, keinerlei Nachteile im Zusammenhang mit der Integration des Rechnungswesens $\mathrm{zu}$ sehen. ${ }^{138}$ Zudem übersteigt in sämtlichen zuvor genannten Studien das Ausmaß der mit der Integration in Verbindung gebrachten Vorteile deutlich jenes der Nachteile, sodass insgesamt eine positive Beurteilung der Integration des internen und externen Rechnungswesens seitens der Unternehmenspraxis konstatiert werden kann. ${ }^{139}$

Obwohl die Integrationsvorteile offensichtlich - wie gezeigt - in erster Linie auf Ebene der Kommunikation angesiedelt sind, wurde die verhaltenswissenschaftlich geprägte Sichtweise des Rechnungswesens als Sprache bzw. Kommunikationsmedium in der empirischen Forschung zur Integration des Rechnungswesens bislang noch nicht aufgegriffen.

Auswirkungen der Integration des Rechnungswesens auf den Controllingerfolg

Die soeben skizzierten Vor- und Nachteile wurden jeweils in einem recht allgemein gehaltenen Zusammenhang erfragt und weitestgehend isoliert betrachtet, sodass sie

136 Vgl. Horváth/Arnaout (1997), S. 262f., Hoke (2001), S. 155-157, Haring/Prantner (2005), S. 152, Müller (2006), S. 168ff. sowie Weißenberger/Angelkort (2007b), S. 29-31.

137 Vgl. Horváth/Arnaout (1997), S. 262, Hoke (2001), S. 158-160, Haring/Prantner (2005), S. 152f., Müller (2006), S. 176ff. sowie Weißenberger/Angelkort (2007b), S. 31-33.

138 Vgl. Horváth/Arnaout (1997), S. 29 sowie Haring/Prantner (2005), S. 153.

139 Ein ähnliches Resümee auf Basis ihrer jeweiligen Studienergebnisse ziehen auch Hoke (2001), S. 158, Haring/Prantner (2005), S. 153, Müller (2006), S. 177 sowie Weißenberger/Angelkort (2007b), S. 32. 
lediglich eingeschränkte Erkenntnisse über die (tatsächliche) Vorteilhaftigkeit der Integration des Rechnungswesens liefern. ${ }^{140}$

Eine erstmals stärker fokussierende empirische Analyse der konkreten Auswirkungen der Integration des Rechnungswesens auf den Erfolg der Controllerarbeit liefert die triadisch konzipierte Studie von Weißenberger/Angelkort (2007b) bzw. Angelkort/Sandt/Weißenberger (2008a). Hier konstatieren $75 \%$ der teilnehmenden Manager, dass die Aussagekraft bzw. der Informationsgehalt der Berichterstattung seit der Integration des Rechnungswesens gestiegen ist. ${ }^{141}$ Des Weiteren gibt fast die Hälfte (43\%) der Manager an, dass sich der Einfluss der Controller auf Entscheidungen des Managements seit der Integration des Rechnungswesens erhöht hat. ${ }^{142}$ Aufgrund der retrospektiven Art der Befragung, die zudem keinen Zusammenhang zur individuellen Integrationsintensität der jeweiligen Unternehmen herstellt, sind jedoch auch diese Ergebnisse nur als erste Anhaltspunkte einzustufen. Einen geeigneteren Ansatz bildet in diesem Zusammenhang die ebenfalls durchgeführte dyadische Regressionsanalyse, in der einige signifikante Beziehungen identifiziert werden konnten. ${ }^{143}$ Hier zeigen die ermittelten Regressionskoeffizienten, dass je besser sich die befragten Controller als gewappnet für die Herausforderungen unter IFRS einschätzen, desto höher die Manager zum einen die Aussagekraft der Monatsberichterstattung $(0,45)$ und zum anderen den Einfluss der Controller auf die (eigenen) Managemententscheidungen $(0,33)$ beurteilen, wobei zwischen den beiden letztgenannten Variablen ebenfalls ein positiver Zusammenhang $(0,40)$ besteht. Des Weiteren ergibt sich ein ebenfalls signifikanter Effekt $(0,39)$ der Selbsteinschätzung des IFRS-Know-hows der Controller auf die seitens der Manager beurteilte Aussagekraft der Monatsberichterstattung. Die hier dargestellten Ergebnisse zeigen somit zwar eine positive Wirkung zweier integrationsnaher unabhängiger Variablen (Vorbereitung der Controller für zukünftige Herausforderungen unter IFRS sowie IFRS-Know-how der Controller) auf zwei abhängige Variablen, die den Controllingerfolg erfassen (Reportinggüte und Einfluss der Controller auf Managemententscheidungen). Belastbare Aussagen hin-

140 In den Studien von Haring/Prantner (2005) und Weißenberger/Angelkort (2007b) wird jedoch z.B. explizit zwischen den grundsätzlich erwarteten und den eingetretenen Vor- bzw. Nachteilen differenziert. Bei Müller (2006) erfolgt dies ebenfalls, allerdings nur für die Vorteile der Integration.

Vgl. Weißenberger/Angelkort (2007b), S. 48f.

142 Vgl. Weißenberger/Angelkort (2007b), S. 62.

143 Vgl. auch im Folgenden Angelkort/Sandt/Weißenberger (2008a), S. $190 \mathrm{f}$. 
sichtlich der allgemeinen Vorteilhaftigkeit der Integration des Rechnungswesens hinsichtlich des Erfolgs der Controllerarbeit können jedoch - schon aufgrund der mit 28 Paarantworten nur geringen absoluten Beobachtungszahl - auch hieraus nicht getroffen werden.

Die Studie von Grieshop/Weber (2007) widmet sich mit der Kooperation von Controllerbereich und externem Rechnungswesen ebenfalls einem integrationsrelevanten Themenfeld. Wie die durchgeführte Kovarianzstrukturanalyse unter anderem zeigt, führt eine gute Zusammenarbeit von Controllerbereich und externem Rechnungswesen zu einer höheren Qualität der Controllerleistungen sowohl im Bereich der Führungsunterstützung als auch in der Rationalitätssicherung. Hierdurch ist es den Controllern so das Fazit der Autoren - letztlich möglich, sich erfolgreich als Dienstleister des Managements zu positionieren. ${ }^{144}$

In der Untersuchung von Bramann (2009) wird der Einfluss der externen Rechnungslegung auf das Controlling von Forschung und Entwicklung analysiert. 64\% der Interviewpartner geben hier an, dass die Bilanzierungsvorschriften des IAS 38 unter anderem zu einer Verbesserung der Koordinations-, Planungs- oder Kontrollfunktion des F\&E-Controllings geführt haben. ${ }^{145}$ Aufgrund der funktionalen Fokussierung der Untersuchung ergeben sich hieraus jedoch keine generalisierbaren Erkenntnisse bezüglich der Integrationswirkung auf den (allgemeinen) Controllingerfolg.

Weide (2009) untersucht in seiner Studie den Einfluss der Integration des Rechnungswesens auf den Erfolg des Management Reportings anhand von acht qualitativen Kriterien. ${ }^{146}$ Die Ergebnisse deuten diesbezüglich auf einen insgesamt positiven $\mathrm{Zu}$ sammenhang hin, da 53\% der Beurteilungen der besagten Kriterien positiv ausfallen, während $35 \%$ neutral und $12 \%$ negativ bewertet werden. ${ }^{147}$ Den wichtigsten bzw. eindeutigsten Erfolgsindikator bildet dabei die Transparenz des Management Reportings. So geben $96 \%$ (oder 23 von 24 ) der Unternehmen des Hauptsamples an,

Vgl. Grieshop/Weber (2007), S. 312f.

Vgl. Bramann (2009), S. 231.

Zu diesen Erfolgsindikatoren zählen sachliche Relevanz, zeitliche Relevanz, Vergleichbarkeit im Zeitablauf, Transparenz, Objektivität, Anreizvertrăglichkeit, Flexibilităt und Wirtschaftlichkeit, vgl. Weide (2009), S. 27-31 sowie S. 146ff.

Vgl. Weide (2009), S. 339f. 
dass die Integration des Rechnungswesens zu einer Erhöhung der ReportingTransparenz geführt hat. ${ }^{148}$

Ähnlich wie im Falle der Studie von Weißenberger/Angelkort (2007b) ist jedoch auch hier kritisch anzumerken, dass die geschilderten Ergebnisse auf einer retrospektiven Art der Befragung ${ }^{149}$ basieren und somit womöglich eine im Rückblick verklärte Beurteilung der Integrationsauswirkungen darstellen. Darüber hinaus wird erneut kein Zusammenhang zwischen der individuellen Integrationsintensität der jeweiligen Unternehmen und dem Erfolg des Management Reportings hergestellt. Problematisch erscheint zudem, dass für die Erfolgsbeurteilung überwiegend auf die (Selbst-) Einschätzung der Controller („Wir sind sehr erfolgreich. “150) zurückgegriffen wird. ${ }^{151}$ Die Sichtweise der eigentlichen Berichtsempfänger - der Manager - bleibt hingegen unberücksichtigt, wodurch es zu Ergebnisverzerrungen kommen könnte. ${ }^{152}$

Zusammenfassend kann im Bezug auf den Stand der empirischen Forschung zur Integration des internen und externen Rechnungswesens konstatiert werden, dass die bisher durchgeführten Studien zwar eine Reihe aufschlussreicher Ergebnisse hervorgebracht haben, ohne das Themenfeld jedoch inhaltlich differenziert genug und in methodisch bestmöglicher Weise zu bearbeiten. Die diesbezüglich aufgezeigten Defizite - hierzu zählen insbesondere die teilweise geringen absoluten Stichprobengrößen, die Verwendung wenig leistungsstarker statistischer Analyseverfahren, die Vernachlässigung verhaltenswissenschaftlicher Ansätze, die retrospektive Art der Befragung, das Fehlen integrationsintensitätsabhängiger Analysen sowie die Nichtberücksichti-

Vgl. Weide (2009), S. 315-317. Auch im Rahmen der ebenfalls durchgefuhrten offenen Erfolgsbeurteilung kristallisierte sich bei den Interviewpartnern die Einschătzung heraus, dass sich infolge der Integration des Rechnungswesens insbesondere die Transparenz des Management Reportings erhoht hat, vgl. Weide (2009), S. 303-307.

So ist dem Interviewleitfaden unter anderem folgende Frage zu entnehmen: „Ist das Reporting infolge der integrationsbedingten Verănderungen jetzt 'erfolgreicher' als vorher?" (Weide (2009), S. 380).

150 Weide (2009), S. 306.

151 Für eine Übersicht der Stellenbezeichnungen sämtlicher Interviewpartner vgl. Weide (2009), S. 199.

152 Vor diesem Hintergrund empfiehlt der Autor für zukünftige Forschungsbemühungen eine dyadisch konzipierte Untersuchung, wenngleich er jedoch die Befragung von Controllern und Mitarbeitern des externen Rechnungswesens (statt Managern) anregt. Vgl. Weide (2009), S. 371. Der Kritikpunkt der Selbsteinschätzung der Controller gilt in ăhnlicher Weise für die zuvor dargestellten Studienergebnisse von Grieshop/Weber (2007). 
gung der Managementperspektive - unterstreichen den weiterhin bestehenden Forschungsbedarf und bilden den Ausgangspunkt für die in den folgenden Abschnitten dargestellte eigene Studie. 


\section{Grundlagen der empirischen Untersuchung}

\section{$1 \quad$ Konzeption und Ablauf der empirischen Untersuchung}

\subsection{Untersuchungsziel und Forschungsdesign}

Die eigene empirische Untersuchung bildet den Kern der vorliegenden Arbeit und verfolgt das Ziel der Beantwortung der drei in Abschnitt Al formulierten Forschungsfragen. Diese lassen sich, wie Abbildung 7 zeigt, strukturell den drei Komponenten des House of Controlling zuordnen, welches in Abschnitt B1.2 als konzeptioneller Bezugsrahmen der Controllerarbeit dargestellt wurde.

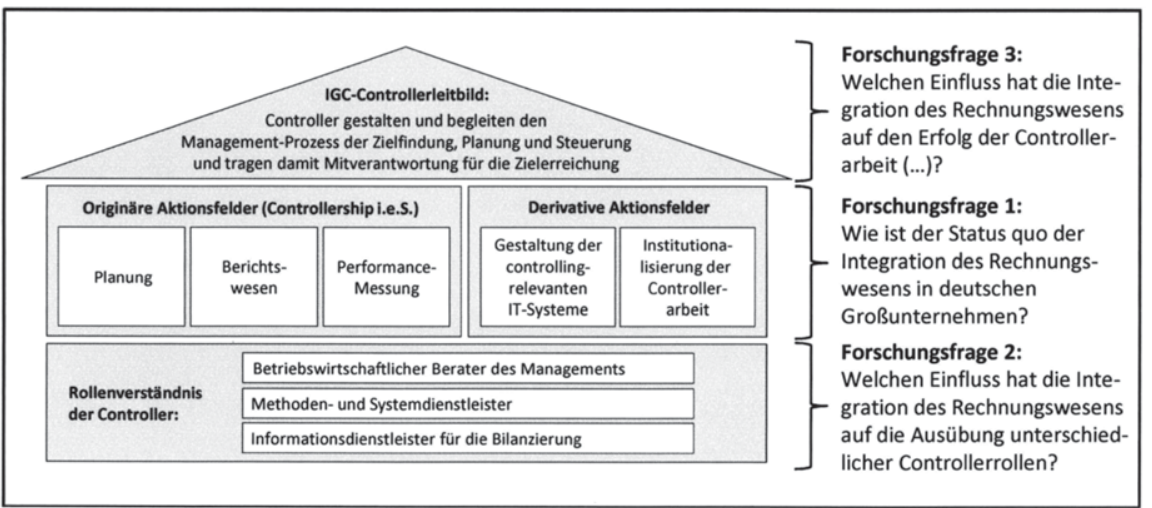

Abbildung 7: Verknüpfung der Forschungsfragen mit dem House of Controlling

So erfolgt die detaillierte Ermittlung des Status quo der Integration des Rechnungswesens in deutschen Großunternehmen (Forschungsfrage 1) untergliedert nach den fünf Aktionsfeldern der Controllerarbeit, während in Forschungsfrage 2 der Einfluss der Integration des Rechnungswesens auf die Ausübung der verschiedenen Controllerrollen untersucht wird. Die Analyse der Auswirkungen einer Integration des Rechnungswesens auf den Erfolg der Controllerarbeit (Forschungsfrage 3) zeigt schließlich auf, wie gut es den Controllern gelingt, Mitverantwortung für die Zielerreichung im Sinne des IGC-Controllerleitbilds zu tragen. 
Eine Besonderheit liegt im dyadischen Forschungsdesign der Untersuchung. ${ }^{153}$ Während die Beantwortung der ersten beiden Forschungsfragen auf der Grundlage der Befragung von Controllern erfolgt, ist für die Beurteilung der Einheitlichkeit der Finanzsprache sowie des Erfolgs der Controllerarbeit im Rahmen der dritten Forschungsfrage idealerweise die Sichtweise der Abnehmer der Controllingdienstleistung - also der Manager als Repräsentanten der Unternehmensführung - heranzuziehen. Auf diese Weise ist es möglich, die Gefahr des Auftretens des so genannten Common Method Bias und der daraus ggf. resultierenden Verzerrungen der Untersuchungsergebnisse zu reduzieren. ${ }^{154}$ Die aufgrund des dyadischen Forschungsdesigns notwendige Beschaffung von Aussagen sowohl seitens der Controller als auch der Manager stellt somit eine besondere Herausforderung im Rahmen der Datenerhebung dar.

\subsection{Durchführung der Datenerhebung}

Die Auswahl der adäquaten Erhebungsmethodik bildet den Ausgangspunkt der Datenerhebung. ${ }^{155}$ Im Rahmen der vorliegenden Untersuchung ist hierbei zu beachten, dass insbesondere aufgrund des zur Beantwortung der dritten Forschungsfrage gewählten kovarianzstrukturanalytischen Forschungsansatzes eine Mindeststichprobengröße von $\mathrm{N}=100$ Fällen erforderlich ist. ${ }^{156}$ Die Erfüllung dieser Anwendungsvoraussetzung wird zudem durch den dyadischen Charakter der vorliegenden Untersuchung erschwert, da hier nur vollständige Dyaden, d.h. Paarantworten eines Controllers und des korrespondierenden Managers aus demselben Unternehmen, berücksichtigt werden können. ${ }^{157}$ Vor diesem Hintergrund stellt eine fragebogengestützte schriftliche Befragung die am besten geeignete Erhebungsmethodik dar, da diese es im Vergleich zu alternativen

153 Für weitere dyadisch konzipierte empirische Untersuchungen innerhalb der betriebswirtschaftlichen Forschung vgl. exemplarisch Homburg/Stock (2001), Bauer (2002), Künkele/Schäffer (2007) sowie Schäffer/Zyder (2007).

154 Vgl. hierzu die Ausfuhrungen in Abschnitt E2.1 der vorliegenden Arbeit.

$155 \mathrm{Zu}$ den gängigen Datenerhebungsmethoden zählen Beobachtungen, Befragungen, Experimente sowie Inhaltsanalysen, vgl. ausfuhrlich Atteslander (2008), Abschnitt II.

$156 \mathrm{Vgl}$. Anderson/Gerbing (1984), S. 170. Zu den methodischen Grundlagen der vorliegenden Untersuchung vgl. Abschnitt C3.

157 Vgl. Homburg/Klarmann (2009), S. 157. 
Methoden - wie etwa der mündlichen Befragung (Interview) - ermöglicht, in kürzerer Zeit mit geringerem Personalaufwand eine größere Zahl von Befragten zu erreichen. ${ }^{158}$

Im Rahmen der Vorbereitung der Datenerhebung wurde zunächst auf Basis einer umfangreichen Literaturanalyse je ein Fragebogen für Controller bzw. Manager entwickelt. Wie Tabelle 3 zeigt, umfasst der Controllerfragebogen neben allgemeinen Fragen zum Unternehmen bzw. zum Unternehmensumfeld Fragestellungen zur Integration des Rechnungswesens, zur Einheitlichkeit der Finanzsprache, zur Ergebnisqualität des Controllings, zum Einfluss des Controllings auf Managemententscheidungen sowie zum Rollenverständnis der Controller.

\begin{tabular}{|l|c|c|c|}
\hline Themengebiet der Fragestellung & $\begin{array}{c}\text { Enthalten im } \\
\text { Controller- } \\
\text { fragebogen }\end{array}$ & $\begin{array}{c}\text { Enthalten im } \\
\text { Manager- } \\
\text { fragebogen }\end{array}$ & $\begin{array}{c}\text { Grundlage für die } \\
\text { Beantwortung von } \\
\text { Forschungsfrage ... }\end{array}$ \\
\hline $\begin{array}{l}\text { Allgemeine Angaben zum Unternehmen/ } \\
\text { Unternehmensumfeld }\end{array}$ & $\checkmark$ & - & 1 \\
\hline $\begin{array}{l}\text { Integration des internen und externen } \\
\text { Rechnungswesens }\end{array}$ & $\checkmark$ & - & $1 ; 2 ; 3$ \\
\hline Einheitlichkeit der Finanzsprache & $\checkmark$ & $\checkmark$ & 3 \\
\hline Ergebnisqualität des Controllings & $\checkmark$ & $\checkmark$ & 3 \\
\hline $\begin{array}{l}\text { Einfluss des Controllings auf Management- } \\
\text { entscheidungen }\end{array}$ & $\checkmark$ & $\checkmark$ & 3 \\
\hline Rollenverständnis der Controller & $\checkmark$ & - & 2 \\
\hline
\end{tabular}

Tabelle 3: Fragebogeninhalte

Der Umfang des Managerfragebogens fällt mit drei Seiten (inklusive Deckblatt) hingegen deutlich kürzer aus als der Controllerfragebogen (neun Seiten), da er lediglich Sachverhalte adressiert, die zur Beantwortung von Forschungsfrage 3 notwendig sind (Einheitlichkeit der Finanzsprache, Ergebnisqualität des Controllings und Einfluss des Controllings auf Managemententscheidungen). Da dieselben Fragen - sprachlich leicht angepasst - ebenfalls im Controllerfragebogen enthalten sind, kann überprüft

158 Neben der kostengünstigen Durchführung zählen ehrlichere Antworten als bei Anwesenheit eines Untersuchenden, überlegtere Antworten (da mehr Zeit zum Ausfullen gegeben ist) sowie die Vermeidung von Interviewfehlern zu den Vorteilen der schriftlichen Befragung. Nachteile liegen dagegen darin begründet, dass die Datenerhebungssituation nicht hinreichend kontrolliert werden kann und somit unklar ist, wer den Fragebogen ausgefullt hat und ob eine Beeinflussung durch externe Faktoren stattgefunden hat. Zudem kommt es bei schriftlichen Befragungen i.d.R. zu vergleichsweise hohen Ausfallquoten, vgl. Atteslander (2008), S. 147 sowie Schnell/Hill/Esser (2008), S. 358-360. 
werden, ob es hinsichtlich der Frage nach dem Einfluss der Integration des Rechnungswesens bzw. der Einheitlichkeit der Finanzsprache auf den Erfolg der Controllerarbeit zwischen Controllern und Managern zu abweichenden Einschätzungen kommt. ${ }^{159}$

Den Abschluss der Fragebogenentwicklung bildete ein Pretest, der die Zielsetzung verfolgte, die Verständlichkeit, die Vollständigkeit, den Aufbau sowie den Umfang des Fragenkatalogs kritisch zu überprüfen. ${ }^{160}$ Hierzu konnten sechs Experten aus der Unternehmenspraxis sowie sechs Wissenschaftler für persönliche Interviews gewonnen werden, deren Anmerkungen und Hinweise bei der Fertigstellung der Fragebögen berücksichtigt wurde. ${ }^{161}$

Die eigentliche Datenerhebung erfolgte im Zeitraum von September bis November 2007. Den Kreis der Untersuchungsobjekte bzw. die Grundgesamtheit der vorliegenden Untersuchung bildeten deutsche Großunternehmen. Ausgangspunkt für die Befragung waren die 1.500 umsatzstärksten Unternehmen mit Sitz in Deutschland, die mindestens 250 Mitarbeiter beschäftigen. Die Unternehmen, die diese Kriterien erfüllen, konnten mithilfe der Hoppenstedt-Firmendatenbank identifiziert werden. Die Begründung für die angesetzte Mindestmitarbeiterzahl liegt darin, dass die Wahrscheinlichkeit des Vorhandenseins - für die Studie notwendiger - institutionalisierter Controllingstrukturen bzw. Controllerstellen mit zunehmender Mitarbeiterzahl steigt. ${ }^{162}$ Im weiteren Verlauf der Datenerhebung wurden einige Unternehmen elimi-

Vgl. hierzu Abschnitt E2.5 der vorliegenden Arbeit.

Die hohe Bedeutung von Pretests im Rahmen von empirischen Erhebungen machen Sudman/ Bradburn (1982) deutlich (S. 283): "If you do not have the resources to pilot-test your questionnaire, don't do the study.“

Insbesondere wurden Formulierungen innerhalb einzelner Items auf Basis der Anmerkungen der Pretest-Teilnehmer geăndert.

Vgl. Küpper (2008), S. 545f.; in der dort angefuhrten empirischen Studie verfügten $72,7 \%$ der untersuchten Unternehmen mit 200-499 Mitarbeitern uber institutionalisierte Controllerstellen. Die in der eigenen Studie gewählte Mindestanzahl von 250 Mitarbeitern fallt zum einen somit in den genannten Bereich von 200-499 Mitarbeitern und lässt daher das Vorliegen entsprechender Controllingstrukturen bei den zu untersuchenden Unternehmen erwarten. Zum anderen knüpft sie an die formale Abgrenzung des Handelsgesetzbuchs sowie der Europäischen Union an. Gemäß $\$ 267$ Absatz 3 HGB gelten große Kapitalgesellschaften als solche, wenn sie mindestens zwei von drei Größenkriterien erfullen, wobei wiederum eines dieser Kriterien in einer Mindestanzahl von 250 Arbeitnehmern liegt. Auch die Europäische Union empfiehlt in ihrer seit dem 01.01.2005 gültigen Abgrenzung von kleinen und mittleren Unternehmen (KMU) eine KMU-Obergrenze von 249 Mitarbeitern, sodass Unternehmen ab 250 Mitarbeitern als Großunternehmen zăhlen, vgl. Europäische Union (2003). 
niert (z.B. im Falle von Doppeleinträgen innerhalb der Datenbank aufgrund verschiedener Rechtsformkonstrukte, Mehrfachzuständigkeiten einzelner Ansprechpartner bei verbundenen Unternehmen, Unternehmen ohne eigenes Controlling oder Unternehmen, deren Firmenpolitik eine Studienteilnahme grundsätzlich ausschließt), sodass sich eine bereinigte Grundgesamtheit von 1.269 Unternehmen ergab.

Um möglichst verallgemeinerbare Erkenntnisse hinsichtlich der zu beantwortenden Forschungsfragen generieren zu können, wurde die Erhebung branchenübergreifend durchgeführt. ${ }^{163}$ Zum Personenkreis der Befragten zählten - wie bereits erwähnt aufgrund des dyadischen Forschungsdesigns der Untersuchung sowohl Controller als auch Manager und somit zwei Gruppen von Unternehmensvertretern. Zunächst erfolgte der Versuch einer telefonischen Kontaktaufnahme mit den Controllingleitern der zu untersuchenden Unternehmen mit der Zielsetzung, für eine Teilnahme an der Untersuchung zu werben. Im zweiten Schritt wurden - auch in den Fällen, in denen der telefonische Kontakt nicht zustande gekommen war - jeweils beide Fragebögen per E-Mail an die Controllingleiter gesendet, begleitet von der Bitte, den eigenen Fragebogen ausgefüllt zurückzusenden und den Managerfragebogen an denjenigen ergebnisverantwortlichen Manager (z.B. Vorstand, Geschäftsführer oder Bereichsleiter) im Unternehmen zur Bearbeitung weiterzuleiten, zu dem der regelmäßigste Kontakt besteht. ${ }^{164}$

$\mathrm{Da}$ eine hohe Rücklaufquote einen erfolgskritischen Faktor für die empirische Untersuchung darstellt, wurde versucht, die angesprochenen Controller mithilfe von Incentivierungsmaßnahmen zur Teilnahme zu motivieren. So wurde den Controllern für die Rücksendung der Fragebögen neben einem individuellen BenchmarkingBericht auf Basis der Studienergebnisse ein Fachbuch aus dem Themenfeld der Integration des Rechnungswesens sowie zwei Gratisausgaben einer Fachzeitschrift in Aussicht gestellt. Des Weiteren wurde nach Ablauf der zweiwöchigen Antwortfrist eine E-Mail-basierte Nachfassaktion durchgeführt, in deren Zuge alle säumigen Unternehmensvertreter noch einmal um Teilnahme an der Untersuchung gebeten wurden.

163 Banken, Versicherungen sowie sonstige Finanzdienstleister werden jedoch aufgrund der branchenspezifischen Besonderheiten sowie der speziellen für diese Unternehmen geltenden Rechnungslegungsvorschriften nicht berücksichtigt. Wie die Ausfuhrungen in Abschnitt B4.1 der vorliegenden Arbeit zeigen, liegt hierin eine übliche Vorgehensweise im Rahmen der empirischen Forschung zum Themenfeld der Integration des Rechnungswesens. 
Der Incentivierung sowie der Nachfassaktion kommt hinsichtlich des Untersuchungserfolgs eine hohe Bedeutung zu, da sie darauf abzielen, den oben genannten Nachteil der schriftlichen Befragung - die hohen Ausfallquoten - abzumildern.

Zur Sicherstellung der Datenqualität wurden alle eingehenden Fragebögen noch während der laufenden Datenerhebung hinsichtlich offensichtlich fehlerhafter Antworten (z.B. zwei gesetzte Kreuze innerhalb einer Rating-Skala) bzw. fehlender Angaben (so genannte 'missing values') ${ }^{165}$ überprüft. Falls notwendig, wurden die entsprechenden Teilnehmer erneut kontaktiert und um eine Korrektur bzw. ein Nachreichen der fehlenden Antworten gebeten. ${ }^{166}$

Auf die Durchführung eines Nonresponse-Bias-Tests ${ }^{167}$ wurde im Rahmen der Datenerhebung verzichtet. Da die Erzielung möglichst zahlreicher vollständiger Dyaden zur Ermöglichung der kovarianzstrukturanalytischen Beantwortung der dritten Forschungsfrage angestrebt wurde, wurden intensive Nachfassmaßnahmen durchgeführt, die als nicht zufällig verteilt einzustufen sind und somit einer sinnvollen Testdurchführung entgegenstehen. ${ }^{168}$

\section{Datengrundlage}

\subsection{Rücklaufquoten}

Hinsichtlich der Datengrundlage wird im Folgenden zwischen dem Rücklauf der Gesamtheit aller Controllerfragebögen (= Hauptstichprobe) und dem Rücklauf vollständiger Dyaden, d.h. Paarantworten eines Controllers und des korrespondierenden Managers aus demselben Unternehmen (= dyadische Stichprobe), unterschieden.

165 Vgl. hierzu Schnell/Hill/Esser (2008), S. 468.

166 Diese zeitintensive und aufwändige Maßnahme erschien angebracht, da es bei der Beantwortung der Fragen vereinzelt zu unbeabsichtigten 'Doppelkreuzen' bzw. Lücken kam.

Der Nonresponse-Bias-Test untersucht, ob es zwischen frühzeitig und im späteren Verlauf einer Untersuchung zurückgesendeten Fragebögen zu signifikanten Unterschieden im Antwortverhalten kommt. Dem Test liegt die Annahme zu Grunde, dass Unternehmen, die erst spät antworten, hinsichtlich ihres Antwortverhaltens tendenziell den nicht antwortenden Unternehmen ähnlich sind, vgl. grundlegend Armstrong/Overton (1977).

168 Für eine analoge Argumentation hinsichtlich des Verzichts auf Durchfuhrung eines NonresponseBias-Tests im Rahmen einer dyadischen Untersuchung vgl. Bauer (2002), S. 152. 
Insgesamt lieferte die Datenerhebung einen Rücklauf von 221 Controllerfragebögen, von denen 214 auswertbar waren. Dies entspricht einer effektiven Rücklaufquote von $16,9 \%$ bezogen auf die bereinigte Grundgesamtheit von 1.269 Unternehmen. Zudem gingen 152 Managerfragebögen ein, von denen 149 für Analysezwecke geeignet waren. Bezogen auf die auswertbaren Controllerfragebögen - denn nur im Falle einer eigenen Teilnahme haben die Controller den zweiten Fragebogen an den entsprechenden Manager weitergeleitet - entspricht dies einer Quote von 70,1\%. Die sich daraus ergebenden 149 auswertbaren Dyaden machen bezogen auf die bereinigte Grundgesamtheit eine dyadische Rücklaufquote von $11,7 \%$ aus. Sowohl die effektive Rücklaufquote der Hauptstichprobe als auch die Rücklaufquote der dyadischen Stichprobe können als sehr positiv beurteilt werden. ${ }^{169}$

In Abbildung 8 wird zum einen der Teilmengencharakter der dyadischen Stichprobe im Hinblick auf die Hauptstichprobe veranschaulicht. Zum anderen zeigt die Abbildung, dass die ersten beiden Forschungsfragen anhand der Controllerantworten auf Basis der Hauptstichprobe beantwortet werden, während der Beantwortung von Forschungsfrage 3 die dyadische Stichprobe zu Grunde liegt.

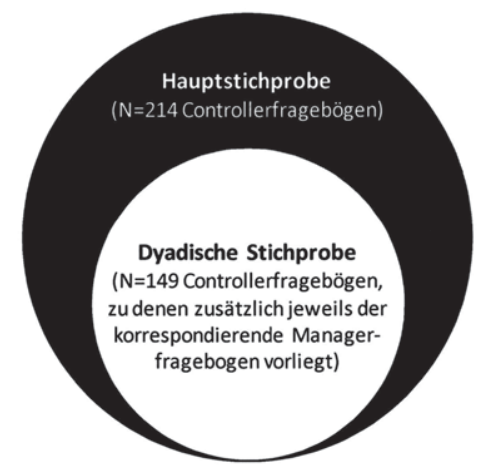

Datengrundlage für die Beantwortung der Forschungsfragen 1 und 2

$\square$ Datengrundlage für die Beantwortung der Forschungsfrage 3

Abbildung 8: Stichprobenabgrenzung

169 Innerhalb der empirischen Controllingforschung sei zu Vergleichszwecken exemplarisch auf die effektiven Rücklaufquoten bei Bauer (2002), S. 152, in Höhe von 13,9\% sowie bei Spillecke (2006), S. 77, in Höhe von 12,5\% verwiesen. Die dyadische Rücklaufquote bei Bauer (2002), S. 153, belief sich auf 9,99\%. Künkele/Schäffer (2007), S. 80, erzielten eine dyadische Rücklaufquote von $12,5 \%$. 
Diese Vorgehensweise liegt darin begründet, dass für das im Rahmen der dritten Forschungsfrage entwickelte Kovarianzstrukturmodell sowohl auf Controllerantworten als auch auf die korrespondierenden Managerantworten zurückgegriffen wird und somit ausschließlich vollständige Dyaden für die Analyse berücksichtigt werden können.

\subsection{Stichprobenmerkmale}

Aufgrund der oben dargestellten Relevanz sowohl der Hauptstichprobe als auch der dyadischen Stichprobe für die Untersuchung werden im Folgenden die wesentlichen Merkmale beider Stichproben dargestellt.

Hinsichtlich der Branchenzusammensetzung zeigt Abbildung 9, dass sowohl in der Hauptstichprobe als auch in der dyadischen Stichprobe Unternehmen aus den Branchen 'Eisen, Stahl, Maschinenbau', 'Chemie, Pharma, Gesundheit' sowie 'Handel' am stärksten vertreten sind. ${ }^{170}$

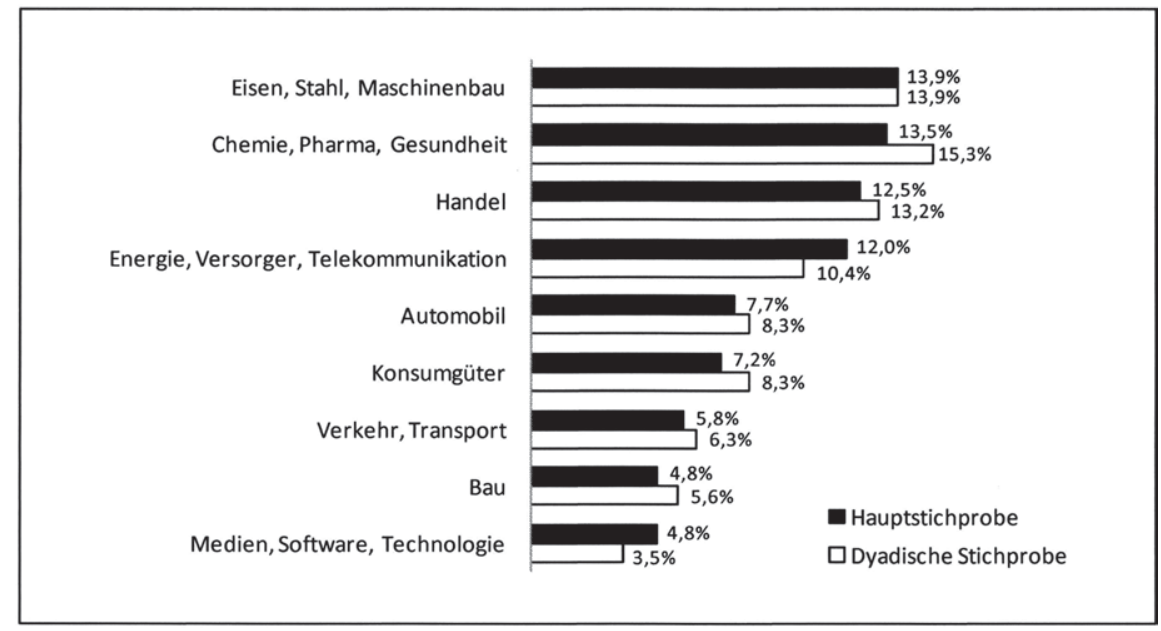

Abbildung 9: Branchenzusammensetzung der Stichproben

$170 \mathrm{Zu}$ beachten ist in diesem Zusammenhang, dass innerhalb der Hauptstichprobe 17,8\% und innerhalb der dyadischen Stichprobe 15,3\% der Unternehmen bezüglich ihrer Branchenzugehörigkeit die Sammelkategorie 'Sonstige' gewăhlt haben. Ein möglicher Erklärungsansatz hierfür könnte darin liegen, dass sich die Geschäftstätigkeit insbesondere von Großunternehmen über verschiedene Geschäftsfelder erstreckt, sodass keine eindeutige Branchenzuordnung möglich war. 
Die Branchenzusammensetzung weist einen hohen Grad an Heterogenität innerhalb der beiden Stichproben auf, woraus sich tendenziell eine hohe Aussagekraft der auf dieser Datengrundlage generierten Ergebnisse ergibt. Branchenspezifische Auswertungen sind aufgrund der pro Branche geringen Fallzahl jedoch nicht möglich.

Ein Chi-Quadrat-Anpassungstest ${ }^{171}$ auf Basis eines Signifikanzniveaus von $5 \%$ ergab keine signifikanten Unterschiede hinsichtlich der Branchenzusammensetzung zwischen der Hauptstichprobe und der dyadischen Stichprobe $(p=0,986)$.

Als Konsequenz der vorgenommenen Abgrenzung der Grundgesamtheit ${ }^{172}$ beinhaltet die Hauptstichprobe - und aufgrund ihres Teilmengencharakters folglich auch die dyadische Stichprobe - ausschließlich Großunternehmen. Die Verteilungsparameter der Größenkriterien Umsatz, Bilanzsumme und Mitarbeiterzahl sind für beide Stichproben in Tabelle 4 dargestellt.

\begin{tabular}{|c|c|c|c|c|c|c|c|}
\hline & & $\mathbf{N}$ & MW & SA & $\begin{array}{l}\text { Unteres } \\
\text { Quartil }\end{array}$ & Median & $\begin{array}{l}\text { Oberes } \\
\text { Quartil }\end{array}$ \\
\hline \multirow{3}{*}{ 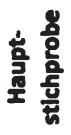 } & Umsatz (Mio. EUR) & 211 & 4.511 & 10.278 & 544 & 1.000 & 2.800 \\
\hline & Bilanzsumme (Mio. EUR) & 180 & 5.426 & 16.969 & 387 & 800 & 2.588 \\
\hline & Mitarbeiter & 212 & 16.548 & 48.106 & 1.600 & 3.778 & 10.000 \\
\hline \multirow{3}{*}{ 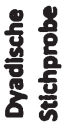 } & Umsatz (Mio. EUR) & 146 & 4.015 & 9.761 & 530 & 978 & 2.254 \\
\hline & Bilanzsumme (Mio. EUR) & 126 & 4.503 & 13.834 & 350 & 795 & 2.211 \\
\hline & Mitarbeiter & 148 & 16.137 & 51.799 & 1.556 & 3.825 & 9.375 \\
\hline
\end{tabular}

Tabelle 4: GrőBenkriterien der Stichproben

Zur Überprüfung, ob sich die beiden Stichproben hinsichtlich der Größenverteilung voneinander unterscheiden - denkbar wäre z.B., dass tendenziell eher die Manager der kleineren Unternehmen antworten, da diese einer vergleichsweise geringeren Arbeitsbelastung unterliegen - wurde für alle drei Kriterien ein Mann-Whitney-UTest ${ }^{173}$ durchgeführt. Es zeigt sich jedoch weder beim Umsatz $(p=0,656)$ noch bei der Bilanzsumme $(p=0,803)$ oder der Mitarbeiterzahl $(p=0,857)$ ein signifikanter Unterschied in der zentralen Tendenz der jeweiligen Verteilungen.

171 Zur Methodik des Chi-Quadrat-Anpassungstests vgl. Janssen/Laatz (2007), S. $561 \mathrm{ff}$.

172 Vgl. hierzu die Ausfuhrungen in Abschnitt C1.2 der vorliegenden Arbeit.

173 Vgl. hierzu die Ausfuhrungen in Abschnitt C3.2 der vorliegenden Arbeit. 
Neben den allgemeinen Stichprobenmerkmalen der Branchenzusammensetzung und der Unternehmensgröße sind im Rahmen der vorliegenden Arbeit zudem die Kriterien der Börsennotierung und des führenden Rechnungslegungsstandards der untersuchten Unternehmen von Interesse. ${ }^{174}$ Wie Abbildung 10 zeigt, ist gut ein Viertel $(27,1 \%)$ der Unternehmen der Hauptstichprobe börsennotiert. Zudem bilden die IFRS bei der Mehrheit der Unternehmen (54,5\%) den führenden Rechnungslegungsstandard, gefolgt vom HGB $(38,0 \%)$ und den US-GAAP $(7,0 \%) .{ }^{175}$

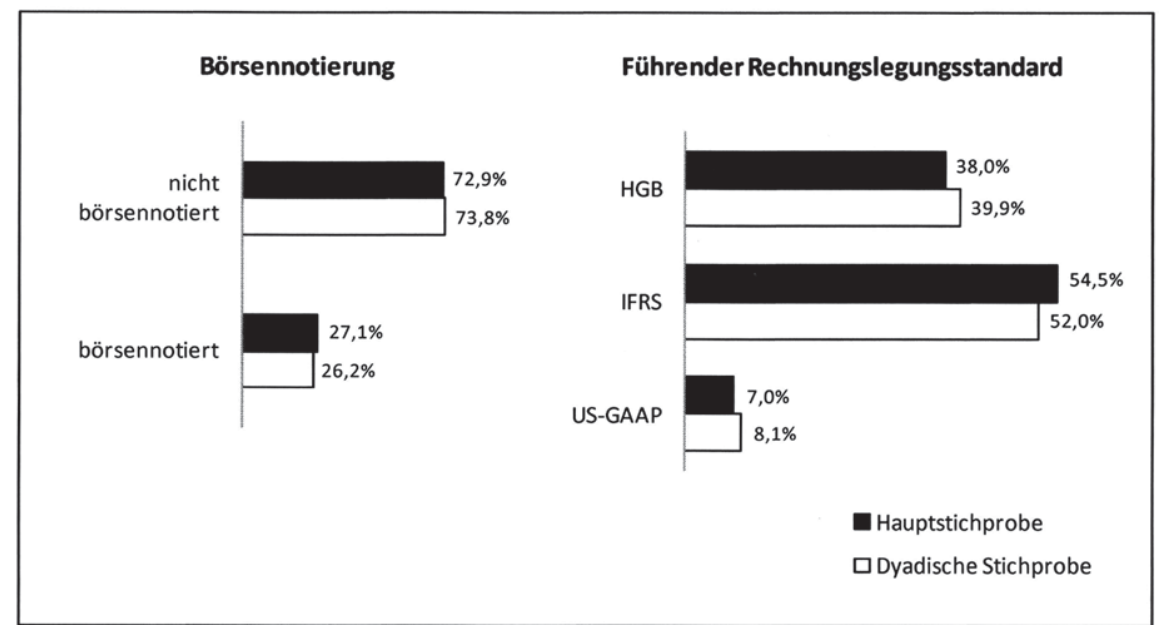

Abbildung 10: Stichprobenmerkmale 'Börsennotierung' und 'Führender Rechnungslegungsstandard'

Ein Chi-Quadrat-Anpassungstest auf Basis eines Signifikanzniveaus von 5\% ergab weder hinsichtlich des Kriteriums der Börsennotierung $(p=0,799)$ noch des führenden Rechnungslegungsstandards $(p=0,758)$ einen signifikanten Unterschied zwischen der Hauptstichprobe und der dyadischen Stichprobe.

Da zwischen den beiden Stichproben hinsichtlich ihrer zentralen Merkmale somit wie gezeigt keine signifikanten Unterschiede vorliegen, kann davon ausgegangen

174 So weisen die untersuchten Unternehmen in Abhängigkeit dieser beiden Kriterien beispielsweise jeweils unterschiedlich starke Integrationsintensitäten des internen und externen Rechnungswesens auf, vgl. Abschnitt D2.3 der vorliegenden Arbeit.

175 Die zur Erreichung der Summe von $100 \%$ fehlenden $0,5 \%$ fallen auf die aus Gründen der Übersichtlichkeit nicht aufgefuhrte Kategorie 'Sonstiger Rechnungslegungsstandard'. 
werden, dass es zu keinen bedeutsamen Verzerrungen zwischen den Ergebnissen der Forschungsfragen 1 und 2 auf der einen Seite und der Forschungsfrage 3 auf der anderen Seite kommt.

Eine statistische Überprüfung der Repräsentativität beider Stichproben bezogen auf die Grundgesamtheit der 1.269 im Rahmen der Untersuchung berücksichtigten Unternehmen war hingegen im vorliegenden Fall nicht möglich, da die verwendete Datenbank das hierfür notwendige Datengerüst nicht in erforderlicher Genauigkeit enthielt. So waren beispielsweise die Umsatzangaben insbesondere im Falle der nicht kapitalmarktorientierten Unternehmen häufig auf einem älteren Stand. Ein Abgleich mit der Verteilung der - einheitlich auf das vorige Geschäftsjahr bezogenen und somit durchweg aktuellen - Umsatzangaben innerhalb der zurückerhaltenen Fragebögen wäre daher wenig sinnvoll gewesen. Die oben dargestellten Stichprobenmerkmale deuten aber dennoch auf eine heterogene Zusammensetzung der Stichproben hin, sodass tendenziell von einer hohen Aussagekraft der erhobenen Daten ausgegangen werden kann.

\section{Methodische Grundlagen der Untersuchung}

Zur Analyse der erhobenen Daten werden im Rahmen der vorliegenden Arbeit - neben deskriptiven Auswertungen - Mittelwertvergleiche, Kreuztabellen sowie eine Kovarianzstrukturanalyse angewendet. Neben einigen Hinweisen zum Skalenniveau der Untersuchungsdaten werden im Folgenden die methodischen Grundlagen dieser Verfahren, inklusive der für die Kovarianzstrukturanalyse notwendigen Konstruktmessung, dargestellt.

\subsection{Skalenniveau der Untersuchungsdaten}

Daten bilden die Grundlage jeder statistischen Analyse und sind das Resultat von Messungen. Der Informationsgehalt der Daten sowie die damit einhergehende Anwendbarkeit von unterschiedlichen Rechenoperationen hängen dabei vom Skalenniveau des für die Messung verwendeten Skalentyps ab. Diesbezüglich wird zwischen Nominal-, Ordinal-, Intervall- sowie Ratioskalen unterschieden, wobei die Nominalskala das niedrigste Messniveau besitzt, während die auf einer Ratioskala gemessenen 
Daten den höchsten Informationsgehalt besitzen und somit die umfangreichsten Rechenoperationen ermöglichen. ${ }^{176}$

Die Daten der vorliegenden Untersuchung wurden überwiegend mithilfe einer sechsstufigen Rating-Skala ${ }^{177}$ gemessen, deren Antwortabstufung jeweils fragenspezifisch ausgestaltet ist. ${ }^{178}$ Grundsätzlich liefern Rating-Skalen lediglich ordinalskalierte Messwerte, ${ }^{179}$ sodass hier beispielsweise die Bildung von Mittelwerten - ähnlich wie bei Schulnoten - streng genommen nicht zulässig ist. ${ }^{180}$ Ist die Anzahl der Antwortabstufungen - wie im vorliegenden Fall mit sechs Ausprägungen - ausreichend hoch, kann jedoch unterstellt werden, dass die Untersuchungsteilnehmer die Abstände innerhalb der Skala als gleiche Intervalle interpretieren. ${ }^{181}$ Die Messwerte werden dann oftmals - wie auch in den nachfolgenden quantitativen Analysen - wie Intervalldaten gehandhabt. $^{182}$

\subsection{Mittelwertvergleiche}

Anhand von Mittelwertvergleichen kann untersucht werden, ob die zwischen zwei (Teil-)Stichproben auftretenden Mittelwertunterschiede auf zufällige Schwankungen zurïckzuführen sind oder ob ein Einfluss der unabhängigen Variablen auf die Höhe der Mittelwerte der abhängigen Variablen vermutet werden kann. ${ }^{183}$ Für die Analyse

Vgl. ausfuhrlich Bortz/Döring (2006), S. 67-70, Backhaus et al. (2008), S. 8-10, Schnell/Hill/ Esser (2008), S. 144f. sowie Mayer (2009), S. 71.

$\mathrm{Zu}$ unterschiedlichen Varianten von Rating-Skalen sowie deren Vor- bzw. Nachteilen vgl. Bortz/Döring (2006), S. 176ff. sowie Bühner (2006), S. 54-56. Häufig werden Rating-Skalen falschlicherweise auch als Likert-Skalen bezeichnet. Bei einer Likert-Skala handelt es sich jedoch um das Ergebnis eines 1932 von Rensis Likert entwickelten Skalierungsverfahrens, das auch als 'Methode der summierten Ratings' bezeichnet wird. Es verfolgt das Ziel, aus einer Vielzahl (ublicherweise ca. 100) von auf einer Rating-Skala gemessenen Items diejenigen Items zu identifizieren, deren Antworten mit den Antworten der ubrigen Items korrelieren und die somit nur eine Dimension erfassen. Das Ergebnis dieser Itemanalyse bildet die endgültige Likert-Skala: eine bis zu 20 oder 30 Items umfassende Fragenbatterie. Vgl. Likert (1932), Bortz/Döring (2006), S. 224, Greving (2007), S. 66 und 73ff., Schnell/Hill/Esser (2008), S. 187-191 sowie Mayer (2009), S. 87f.

Vgl. Abschnitte D1 sowie E2.2 der vorliegenden Arbeit.

Vgl. Mayer (2009), S. 83.

Vgl. Schnell/Hill/Esser (2008), S. 145f.

Vgl. Bortz/Döring (2006), S. 70 und 181f., Mayer (2009), S. 83 sowie mit besonderem Augenmerk auf latente Variablen Homburg/Klarmann (2006), S. 733 und Schnell/Hill/Esser (2008), S. 147.

Vgl. Backhaus et al. (2008), S. 9.

Vgl. Bühl (2006), S. 299 sowie Janssen/Laatz (2007), S. 334. 
stehen grundsätzlich zwei alternative Verfahren zur Verfügung, die sich hinsichtlich der Anforderungen an die zu Grunde liegenden Daten unterscheiden:

- Der t-Test zählt zu den parametrischen Testverfahren, da er eine Normalverteilung der abhängigen Variablen in der Grundgesamtheit voraussetzt. Darüber hinaus müssen die Daten der abhängigen Variablen mindestens intervallskaliert sein. $^{184}$

- Der Mann-Whitney-U-Test setzt als nichtparametrischer (auch: verteilungsfreier) Test keine Normalverteilung voraus und kann auch bei Ordinalskalenniveau der abhängigen Variablen eingesetzt werden. ${ }^{185}$

$\mathrm{Da}$ in der vorliegenden Untersuchung - wie recht häufig im sozialwissenschaftlichen Bereich - überwiegend keine normalverteilten Daten vorliegen, ${ }^{186}$ wird zu Analysezwecken auf den Mann-Whitney-U-Test zurückgegriffen. Dieser ist zwar im Vergleich zum t-Test weniger trennscharf, da er auf Rangziffern statt auf die Variablenwerte selbst zurückgreift und es somit zu einem Informationsverlust kommt. ${ }^{187}$ Seine Anwendung wird aber im Falle der Nichterfüllung der Anwendungsbedingungen parametrischer Tests empfohlen, da ansonsten die Gefahr besteht, dass es zu falschen Testergebnissen kommt.

Während der Mann-Whitney-U-Test für den Vergleich von zwei unabhängigen Stichproben herangezogen wird, ist bei abhängigen Stichproben dagegen auf den ebenfalls nichtparametrischen - Wilcoxon-Test zurückzugreifen, der auf einer Rang-

Vgl. ausfuhrlich Bortz (2005), S. 140ff., Bühl (2006), S. 300ff. sowie Janssen/Laatz (2007), S. $347 \mathrm{ff}$.

In der Literatur wird hier mitunter nicht von einem Mittelwertvergleich gesprochen - das arithmetische Mittel ist fur ordinalskalierte Messwerte nicht skaliert -, sondern von einem Vergleich hinsichtlich der zentralen Tendenz von zwei Stichproben, vgl. Bortz (2005), S. 150. Da in der vorliegenden Untersuchung die anhand der sechsstufigen Rating-Skala erhobenen Ordinaldaten wie metrische Werte gehandhabt werden (vgl. hierzu die Ausfuhrungen im vorangegangenen Abschnitt der vorliegenden Arbeit), werden dennoch Mittelwertunterschiede betrachtet. Vgl. zum Mann-Whitney-U-Test ausfuhrlich Bortz (2005), S. 150ff., Bühl (2006), S. 313ff. sowie Janssen/ Laatz (2007), S. $571 \mathrm{ff}$. sowie Janssen/Laatz (2007), S. 569f.) fur sämtliche in der empirischen Analyse berucksichtigten Variablen. 
reihe der absoluten Wertepaardifferenzen basiert. ${ }^{188}$ Von abhängigen (auch: verbundenen, gepaarten) Stichproben wird ausgegangen, wenn sich jedem Wert der einen Stichprobe in eindeutiger Weise genau ein Wert der anderen Stichprobe zuordnen lässt. ${ }^{189}$ Sowohl dem Mann-Whitney-U-Test als auch dem Wilcoxon-Test liegen die folgenden Hypothesen zu Grunde: ${ }^{190}$

$H_{0}$ : Die beiden betrachteten Stichproben stammen aus der gleichen Grundgesamtheit, d.h. der Mittelwertunterschied ist zufällig zu Stande gekommen.

$H_{l}$ : Die beiden betrachteten Stichproben stammen aus verschiedenen Grundgesamtheiten, d.h. der Mittelwertunterschied ist nicht zufällig zu Stande gekommen.

Die Entscheidung, ob die Nullhypothese $\mathrm{H}_{0}$ verworfen und die Alternativhypothese $\mathrm{H}_{1}$ angenommen werden kann, wird anhand des aus der Analyse resultierenden Signifikanzniveaus p getroffen. Diesbezüglich wird in der vorliegenden Arbeit - auch für die übrigen statistischen Verfahren - die folgende Abgrenzung verwendet: ${ }^{191}$

- $\mathrm{p} \geq 0,05:$ nicht signifikant

- $\mathrm{p}<0,05: \quad$ signifikant $(*)$

- $\mathrm{p}<0,01$ : hoch signifikant $(* *)$

- $\mathrm{p}<0,001$ : höchst signifikant $(* * *)$

Das Signifikanzniveau stellt die so genannte Irrtumswahrscheinlichkeit dar, d.h. die Wahrscheinlichkeit, dass auf Basis des gefundenen Stichprobeneffektes fälschlicherweise die Alternativhypothese $\mathrm{H}_{1}$ angenommen wird, obwohl in der Grundgesamtheit die Nullhypothese $\mathrm{H}_{0}$ gilt. ${ }^{192}$ Von einem signifikanten Mittelwertunterschied wird

Vgl. ausführlich Bortz (2005), S. 153f., Bühl (2006), S. 319ff. sowie Janssen/Laatz (2007), S. 583ff. Bei der Durchführung des parametrischen t-Tests wird ebenfalls zwischen abhängigen und unabhängigen Stichproben unterschieden, vgl. Bortz (2005), S. 143, Bühl (2006), S. 299 sowie Janssen/Laatz (2007), S. 353. Vgl. Bortz (2005), S. 143, Bühl (2006), S. 113 sowie Janssen/Laatz (2007), S. 560.

Vgl. Janssen/Laatz (2007), S. 115.

Vgl. Bortz/Döring (2006), S. 26f.

192 Diese irrtümliche Annahme der Alternativhypothese wird als 'Fehler 1. Art' bzw. 'Alpha-Fehler' bezeichnet. Hingegen wird vom 'Fehler 2. Art' bzw. 'Beta-Fehler' gesprochen, wenn ein tatsächlich vorhandener Effekt durch einen Signifikanztest nicht entdeckt wird, vgl. Bortz (2005), S. 110ff., Bühl (2006), S. 115, Janssen/Laatz (2007), S. 343f. sowie Schnell/Hill/Esser (2008), S. $452 \mathrm{f}$. 
folglich ausgegangen, wenn die Irrtumswahrscheinlichkeit einer unberechtigten Annahme der Alternativhypothese unterhalb der 5\%-Grenze liegt und somit sehr niedrig ist. Für ein hoch bzw. höchst signifikantes Ergebnis liegt die Signifikanzschwelle mit $1 \%$ bzw. $0,1 \%$ entsprechend noch niedriger.

Hinsichtlich der Interpretation von Signifikanztests sei an dieser Stelle darauf hingewiesen, dass ein statistisch signifikantes Ergebnis nicht gleichbedeutend ist mit einem starken oder bedeutsamen Effekt. So ist bei sehr hoher Fallzahl nahezu jeder Effekt statistisch signifikant, kann aber gleichwohl so schwach sein, dass er praktisch und theoretisch bedeutungslos ist. ${ }^{193}$

\subsection{Kreuztabellen und Chi-Quadrat-Unabhängigkeitstest}

Kreuztabellen dienen der Analyse von Zusammenhängen zwischen zwei oder mehr nominalskalierten Variablen. Im einfachsten Fall einer Gegenüberstellung zweier dichotomer Variablen ergibt sich eine zweidimensionale Kreuztabelle mit vier Zellen, die auch als 'Vier-Felder-Tafel' bezeichnet wird. ${ }^{194}$ Anhand eines Vergleichs der absoluten bzw. relativen Häufigkeiten der einzelnen Zellen mit den jeweiligen Randverteilungen lassen sich erste Hinweise auf einen möglichen Zusammenhang der untersuchten Variablen gewinnen. Für den Fall, dass die Kreuztabellierung eine solche Vermutung unterstützt, kann im Rahmen einer Kontingenzanalyse mithilfe des ChiQuadrat-Unabhängigkeitstests geprüft werden, ob die beiden Variablen voneinander unabhängig sind oder nicht. ${ }^{195}$ Dieser Überprüfung liegen - ähnlich wie bei der Durchführung eines Mittelwerttests - zwei Hypothesen zu Grunde:

$H_{0}$ : Zwischen den betrachteten Variablen besteht kein Zusammenhang.

$H_{I}$ : Zwischen den betrachteten Variablen besteht ein Zusammenhang.

Häufig ist eine der beiden Variablen als abhängig und die andere als unabhängig einzustufen. In diesem Fall wird untersucht, ob die unabhängige Variable einen Einfluss auf die abhängige Variable ausübt. ${ }^{196}$ Als Prüfgröße fungiert üblicherweise der Chi-Quadrat-Wert nach Pearson, der die Stärke der Abweichung der in der Kreuztabel-

Vgl. Janssen/Laatz (2007), S. 344 sowie Schnell/Hill/Esser (2008), S. $452 f$.

Vgl. Atteslander (2008), S. 292 sowie Backhaus et al. (2008), S. 298 und 302.

Vgl. auch im Folgenden Bühl (2006), S. 259f., Janssen/Laatz (2007), S. 262, Atteslander (2008),

S. 292f. sowie Backhaus et al. (2008), S. 306f.

Vgl. Bühl (2006), S. 248. 
le beobachteten von der nach Maßgabe der Nullhypothese erwarteten Verteilung misst. ${ }^{197}$ Bei einem signifikanten Testergebnis - auch hier wird in der Regel eine Irrtumswahrscheinlichkeit von unter $5 \%$ gefordert - wird davon ausgegangen, dass zwischen den untersuchten Variablen ein Zusammenhang besteht, und die Alternativhypothese $\mathrm{H}_{1}$ wird somit als weitestgehend gesichert angenommen. ${ }^{198}$

Für die Durchführung des Chi-Quadrat-Unabhängigkeitstests nach Pearson gilt als Anwendungsvoraussetzung, dass der Anteil der Felder einer Kreuztabelle, deren erwartete Häufigkeit kleiner als fünf ist, maximal $20 \%$ beträgt. Zudem darf keine dieser Häufigkeiten kleiner als eins sein. ${ }^{199}$

\subsection{Konstruktmessung}

\section{Grundlagen der Konstruktmessung}

In der vorliegenden Arbeit werden im Rahmen der Beantwortung der dritten Forschungsfrage komplexe Sachverhalte untersucht, wie z.B. das theoretische Konstrukt 'Einheitlichkeit der Finanzsprache'. Ein theoretisches Konstrukt ist

"an abstract entity which represents the ,true; nonobservational state or nature of a phenomenon "200

und wird, da es sich somit um eine nicht direkt messbare Größe handelt, auch als latente Variable bezeichnet. ${ }^{201}$ Die Verwendung eines Konstrukts im Rahmen der empirischen Analyse erfordert zunächst sowohl dessen Konzeptualisierung, also die Erarbeitung der relevanten Konstruktdimensionen, als auch die hierauf aufbauende Operationalisierung, d.h. die Entwicklung eines geeigneten Messinstruments. ${ }^{202}$ Das Konstrukt wird hierbei anhand von Korrespondenzregeln von der theoretischen Sprachebene auf die Ebene der Beobachtungssprache übertragen und somit unter

Vgl. Janssen/Laatz (2007), S. 263, Atteslander (2008), S. 293 sowie Backhaus et al. (2008), S. $306 f$.

198 Vgl. Janssen/Laatz (2007), S. 262, Atteslander (2008), S. 293 sowie Backhaus et al. (2008), S. $307 \mathrm{f}$.

199 Vgl. Bühl (2006), S. 261, Janssen/Laatz (2007), S. 266 sowie Backhaus et al. (2008), S. 319.

200 Bagozzi/Fornell (1982), S. 24.

201 Vgl. Bollen (1989), S. 11 sowie Homburg/Giering (1996), S. 6.

202 Vgl. Homburg/Giering (1996), S. 5. 
Zuhilfenahme von beobachtbaren Variablen - auch Indikatoren genannt - messbar gemacht. $^{203}$

Hinsichtlich der Konstruktdimensionen kann bei der Konzeptualisierung zwischen ein- und mehrfaktoriellen Konstrukten unterschieden werden. Im einfaktoriellen Fall lassen sich die Indikatoren direkt auf der Konstruktebene verdichten, und das Konstrukt entspricht somit genau einem Faktor. Ein mehrfaktorielles Konstrukt besteht hingegen aus zwei oder mehr Faktoren, wobei ein eindimensionales Konstrukt vorliegt, wenn jeder der Faktoren zur selben theoretischen Dimension des Konstrukts gehört. Ist dies nicht der Fall, so handelt es sich um ein mehrdimensionales Konstrukt, d.h. jede Dimension eines Konstrukts besteht ihrerseits aus mehreren Faktoren. ${ }^{204}$

Bei der Operationalisierung von Konstrukten ist zwischen reflektiven und formativen Messmodellen zu differenzieren. ${ }^{205}$ Einem reflektiven Messmodell liegt die Annahme $\mathrm{zu}$ Grunde, dass die einzelnen Indikatoren ${ }^{206}$ durch die latente Variable kausal verursacht werden. Eine Änderung der Ausprägung der latenten Variablen geht folglich mit einer Änderung der Werte ihrer Indikatoren einher. ${ }^{207}$ Diese stellen - im idealisierten Falle einer fehlerfreien Messung - somit austauschbare Messungen des Konstrukts dar und sind vollständig korreliert. ${ }^{208}$ Im Zuge der Operationalisierung eines reflektiven Messmodells ist daher auf eine möglichst hohe Korrelation der Indikatoren zu achten. Indikatoren, die keine hohe Korrelation aufweisen, sind hingegen zu eliminieren, da sie offensichtlich das betrachtete Konstrukt nicht in ausreichendem Maße 'reflektieren'. ${ }^{209}$ In einem formativen Messmodell wird eine im Vergleich zum reflektiven Fall umgekehrte Wirkungsbeziehung zwischen der latenten Variable und den Indikatoren unterstellt. Diese können hier als 'Bausteine' des Konstrukts interpretiert werden, da das Konstrukt eine Linearkombination der Indikatoren (plus

Vgl. Fassott/Eggert (2005), S. 34.

Vgl. Homburg/Giering (1996), S. 6.

Vgl. grundlegend Bollen/Lennox (1991), MacCallum/Browne (1993), Diamantopoulos/ Winklhofer (2001), Eberl (2004), Fassott/Eggert (2005), Fassott (2006) sowie Christophersen/ Grape (2007).

Die Indikatoren eines reflektiven Messmodells werden in der englischsprachigen Literatur auch als 'effect indicators' bezeichnet, da sie eine Linearkombination (plus Fehlerterm) der latenten Variablen darstellen und somit von ihr abhängig sind, vgl. Bollen/Lennox (1991), S. $305 f$.

Vgl. Eberl (2004), S. 3 sowie Fassott/Eggert (2005), S. 37.

Vgl. Bollen/Lennox (1991), S. 308, Diamantopoulos/Winklhofer (2001), S. 271, Jarvis/ Mackenzie/Podsakoff (2003), S. 200 sowie Fassott/Eggert (2005), S. 37.

Vgl. Bollen/Lennox (1991), S. 308 sowie Zinnbauer/Eberl (2004), S. 4. 
Fehlerterm) darstellt und somit durch sie verursacht wird. ${ }^{210}$ Aus einer Änderung eines Indikators resultiert folglich stets eine Änderung der Ausprägung der latenten Variablen. ${ }^{211}$ Hingegen gilt im Umkehrschluss nicht, dass eine Änderung der latenten Variablen eine Änderungen aller Indikatoren mit sich bringt, da diese untereinander nicht korreliert sein müssen (wenngleich dies jedoch durchaus zulässig ist). ${ }^{212}$

Obwohl formativen Messmodellen in jüngster Zeit eine erhöhte Aufmerksamkeit entgegengebracht wird, ${ }^{213}$ sind sie bislang im Rahmen von empirischen Untersuchungen der betriebswirtschaftlichen Forschung eher selten verwendet worden. ${ }^{214}$ Wie verschiedene Studien ${ }^{215}$ gezeigt haben, ist es in der Vergangenheit bei der Operationalisierung von Konstrukten diesbezüglich häufig zu Fehlspezifikationen gekommen. Diamantopoulos/Riefler/Roth (2008) konstatieren hinsichtlich dieser Problematik:

„Most researchers apply scale development procedures without even questioning their appropriateness for the specific construct at hand [...]. Consequently, misspecification commonly concerns the adoption of reflective indicators where formative indicators (and thus index construction approaches) would be appropriate. "216

Der Wahl des geeigneten Messmodells kommt eine hohe Bedeutung zu, da Fehlspezifikationen unter anderem zu deutlichen Verzerrungen der Parameterschätzungen in Strukturgleichungsmodellen führen können und somit gravierende Konsequenzen hinsichtlich der Interpretation von Modelergebnissen nach sich ziehen. ${ }^{217}$ Die Entscheidung, ob ein reflektives oder ein formatives Messmodell spezifiziert werden

Vgl. MacCallum/Browne (1993), S. 553, Eberl (2004), S. 4, Fassott/Eggert (2005), S. 38. In der englischsprachigen Literatur werden die Indikatoren eines formativen Messmodells daher auch als 'causal indicators' bezeichnet, vgl. Bollen/Lennox (1991), S. 305f. Im Gegensatz zu reflektiven Indikatoren stellen sie keine austauschbaren Messungen eines Konstrukts dar, wie die Autoren treffend formulieren: „Omitting an indicator is omitting a part of the construct" (Bollen/Lennox (1991), S. 308).

Vgl. Jarvis/Mackenzie/Podsakoff (2003), S. 201, Eberl (2004), S. 6, Zinnbauer/Eberl (2004), S. 4f., Fassott/Eggert (2005), S. 38 sowie Christophersen/Grape (2007), S. 105.

Vgl. Diamantopoulos/Winklhofer (2001), S. 271, Jarvis/Mackenzie/Podsakoff (2003), S. 201f., Eberl (2004), S. 6 sowie Fassott/Eggert (2005), S. 38.

213 Vgl. Diamantopoulos (2008), S. 1201.

214 Vgl. Diamantopoulos/Riefler/Roth (2008), S. 1203f.

215 Vgl. exemplarisch Jarvis/Mackenzie/Podsakoff(2003) sowie Fassott (2006).

216 Diamantopoulos/Riefler/Roth (2008), S. 1208.

217 Vgl. Jarvis/Mackenzie/Podsakoff (2003), S. $211 \mathrm{f}$. Zu weiteren Auswirkungen von Fehlspezifikationen vgl. Eberl (2006), S. $654 \mathrm{f}$. 
sollte, kann anhand der in Tabelle 5 dargestellten Entscheidungsfragen zur Kausalitätsbeziehung zwischen Konstrukt und Indikatoren getroffen werden.

\begin{tabular}{|c|c|c|}
\hline Entscheidungsfragen & $\begin{array}{l}\text { Reflektives } \\
\text { Messmodel }\end{array}$ & $\begin{array}{l}\text { Formatives } \\
\text { Messmodell }\end{array}$ \\
\hline \multicolumn{3}{|l|}{$\begin{array}{l}\text { Kausalitätsbeziehung zwischen Konstrukt und } \\
\text { Indikatoren }\end{array}$} \\
\hline $\begin{array}{c}\rightarrow \text { Verläuft die Richtung der Kausalität vom Konstrukt } \\
\text { zum Indikator oder vom Indikator zum Konstrukt? }\end{array}$ & $\begin{array}{l}\text { Vom Konstrukt } \\
\text { zum Indikator }\end{array}$ & $\begin{array}{l}\text { Vom Indikator } \\
\text { zum Konstrukt }\end{array}$ \\
\hline $\begin{array}{l}\text { Würden Veränderungen in der Ausprägung der } \\
\text { Indikatoren eine Veränderung des Konstrukts } \\
\text { verursachen? }\end{array}$ & Nein & Ja \\
\hline $\begin{array}{l}\rightarrow \text { Würden Veränderungen in der Ausprägung des } \\
\text { Konstrukts Veränderungen der Indikatoren } \\
\text { verursachen? }\end{array}$ & Ja & Nein \\
\hline $\begin{array}{l}\rightarrow \text { Sind die Indikatoren Manifestationen oder } \\
\text { definierende Charakteristika des Konstrukts? }\end{array}$ & Manifestationen & $\begin{array}{l}\text { Definierende } \\
\text { Charakteristika }\end{array}$ \\
\hline
\end{tabular}

Tabelle 5: Entscheidungsfragen zur Bestimmung der geeigneten Konstruktspezifikation ${ }^{218}$

\section{Reliabilität und Validität von Messmodellen}

Der Erklärungsgehalt sozialwissenschaftlicher Theorien ist davon abhängig, inwiefern die theoretischen Konstrukte durch Messungen operationalisiert werden können. ${ }^{219}$ In diesem Zusammenhang stellt Fassott (2006) fest:

„Reliable und valide Messmodelle sind eine conditio sine qua non der empirischen Forschung “220

Die Reliabilität (auch: Zuverlässigkeit) einer Messung ist in der klassischen Testtheorie definiert als der Quotient der Varianz der wahren Werte und der Varianz der beobachteten Werte. ${ }^{221}$ Die reflektiven Indikatoren stellen reliable Messungen eines

218 Eigene Darstellung in Anlehnung an Jarvis/Mackenzie/Podsakoff (2003), S. 203 sowie Eberl (2006), S. 658. Zwar finden sich in den hier angefuhrten Literaturquellen weitere Entscheidungsfragen, wie etwa zur Austauschbarkeit der Indikatoren oder zur Beziehung der Indikatoren untereinander. Diese kőnnen jedoch vernachlăssigt werden, da sich „der Kriterienkatalog [...] auf die Frage der Kausalität zwischen Indikator und Konstrukt verdichten lässt. Andere Kriterien zur Entscheidung zwischen formativen und reflektiven Indikatoren sind unnotig“" (Herrmann/ Huber/Kressmann (2006), S. 48).

Vgl. Hildebrandt (1984), S. 41.

220 Fassott (2006), S. 69.

221 Vgl. Schnell/Hill/Esser (2008), S. 151. 
Konstrukts dar, wenn sie einen möglichst geringen Zufallsfehler aufweisen und der wesentliche Teil ihrer Varianz durch das Konstrukt erklärt wird. ${ }^{222}$ Zur Überprüfung der Reliabilität von Messergebnissen existieren in der empirischen Forschung drei verschiedene Standardmethoden: $:^{223}$

- Die Test-Retest-Reliabilität bildet die zeitliche Stabilität von Messergebnissen ab und bezeichnet die Korrelation einer Messung mit einer anhand desselben Messinstruments zu einem späteren Zeitpunkt durchgeführten Vergleichsmessung.

- Die Parallel-Test-Reliabilität greift auf die Korrelation einer Messung mit einer anhand eines äquivalenten Messinstruments zeitgleich durchgeführten Vergleichsmessung zurück.

- Die Interne-Konsistenz-Reliabilität drückt aus, inwieweit die reflektiven Indikatoren austauschbare Messungen eines zu Grunde liegenden Konstrukts darstellen und betrachtet die Korrelation der Indikatoren untereinander.

Da die Überprüfung der beiden erstgenannten Reliabilitäten sehr aufwändig ist, wird in der empirischen Forschung in der Regel lediglich die Interne-Konsistenz-Reliabilität untersucht. ${ }^{224}$ Hinsichtlich der Beurteilung der Reliabilität ist schließlich anzumerken, dass diese nicht nur vom verwendeten Messinstrument, sondern auch von der Zusammensetzung der Menge der Untersuchungsobjekte abhängig ist. Ändert sich diese, so geht hiermit in der Regel auch eine Änderung der Reliabilitätsbeurteilung einher. ${ }^{225}$

Die Validität (auch: Gültigkeit) einer Messung drückt aus, inwiefern das Messinstrument tatsächlich das misst, was es messen soll. Sie bildet somit die konzeptionelle Richtigkeit einer Messung ab. ${ }^{226}$ Die Reliabilität eines Messmodells stellt eine notwendige, jedoch keine hinreichende Bedingung für dessen Validität dar. ${ }^{227}$ Bei der

Vgl. Homburg/Giering (1996), S. 6 sowie Homburg/Pflesser (2000a), S. 420.

Vgl. auch im Folgenden Hildebrandt (1984), S. $41 \mathrm{f}$. sowie Schnell/Hill/Esser (2008), S. $151 \mathrm{f}$.

Vgl. Hildebrandt (1984), S. 42 sowie Homburg/Pflesser (2000a), S. 421.

Vgl. Schnell/Hill/Esser (2008), S. 153.

Vgl. Homburg/Giering (1996), S. 7 sowie Schnell/Hill/Esser (2008), S. 154.

Dies liegt darin begründet, dass für das Vorliegen einer reliablen Messung lediglich ein möglichst geringer Zufallsfehler notwendig ist, wăhrend für die Validităt zusătzlich ein möglichst geringer systematischer Fehler vorliegen muss, vgl. Hildebrandt (1984), S. 42, Homburg/Giering (1996), S. 7 sowie Homburg/Pflesser (2000a), S. 421. 
Messung von Konstrukten werden üblicherweise die folgenden Validitätsarten unterschieden: $:^{228}$

- Die Inhaltsvalidität erfasst, inwieweit die Indikatoren inhaltlich-semantisch mit dem zu erfassenden Konstrukt übereinstimmen und dessen Bedeutungsinhalte und Facetten vollständig abbilden. Da für die Beurteilung der Inhaltsvalidität keinerlei objektiven Kriterien existieren, ist sie vor allem als Leitidee bei der Entwicklung von Messmodellen zu betrachten.

- Die Konstruktvalidität bildet das anspruchsvollste Kriterium zum Nachweis von Validität und beschäftigt sich mit der Beziehung zwischen Konstrukt und Messinstrument. Sie umfasst die drei folgenden Aspekte:

- Konvergenzvalidität beschreibt das Ausmaß, zu dem zwei oder mehr unterschiedliche Messungen desselben Konstrukts übereinstimmen. Im Falle reflektiver Messmodelle sollte daher zwischen den einzelnen Indikatoren eines Konstrukts ein starker Zusammenhang bestehen.

- Diskriminanzvalidität ist das Ausmaß, zu dem sich Messungen unterschiedlicher Konstrukte unterscheiden. Demnach sollte bei reflektiven Messmodellen die Assoziation zwischen zwei Indikatoren desselben Konstrukts stärker sein als die Assoziation zwischen zwei Indikatoren, die unterschiedliche Konstrukte messen.

- Nomologische Validität stellt das Ausmaß dar, in dem theoretisch abgeleitete Zusammenhänge zwischen verschiedenen Konstrukten mit den empirisch nachgewiesenen Beziehungen übereinstimmen. Voraussetzung hierfür ist die Einbindung des Konstrukts in einen übergeordneten theoretischen Rahmen. Die nomologische Validität ist daher lediglich qualitativ überprüfbar.

Gütebeurteilung von Messmodellen

Zur Überprüfung der Reliabilität und Validität von Messmodellen stehen verschiedene quantitative Verfahren zur Verfügung. Hierbei ist zu beachten, dass für formativ

228 Vgl. auch im Folgenden Hildebrandt (1984), S. 42-44, Homburg/Giering (1996), S. 7f., Homburg/Pflesser (2000a), S. 421f. sowie Schnell/Hill/Esser (2008), S. 155ff. 
operationalisierte Konstrukte nicht dieselben Verfahren einsetzbar sind wie für reflektiv operationalisierte Konstrukte. ${ }^{229}$ Da keines der in der vorliegenden Arbeit verwendeten Konstrukte anhand eines formativen Messmodells abgebildet wird, ${ }^{230}$ erfolgt an dieser Stelle lediglich die Darstellung der Gütekriterien für reflektive Messmodelle. ${ }^{231}$ Diese lassen sich in Kriterien der ersten und der zweiten Generation unterteilen: $:^{232}$

- Die Reliabilitäts- und Validitätskriterien der ersten Generation haben ihre Wurzeln in der Psychologie bzw. Psychometrie. Sie dienen ausschließlich der Beurteilung von Messmodellen.

- Die Reliabilitäts- und Validitätskriterien der zweiten Generation basieren auf der konfirmatorischen Faktorenanalyse. Sie gelten als deutlich leistungsstärker, da sie im Gegensatz zu den herkömmlichen Methoden unter anderem die explizite Berücksichtigung von Messfehlern erlauben.

Zunächst werden mit der explorativen Faktorenanalyse, dem Cronbach'schen Alpha und der Item to Total-Korrelation die drei in der vorliegenden Arbeit verwendeten Kriterien der ersten Generation erörtert.

Die explorative (auch: exploratorische) Faktorenanalyse zählt zu den Strukturenentdeckenden multivariaten Verfahren und zielt darauf ab, eine Vielzahl von Indikatoren auf einige wenige Faktoren bzw. Konstrukte zu reduzieren, die die Indikatoren ausreichend gut repräsentieren. ${ }^{233}$ Anders als bei der konfirmatorischen Faktorenanalyse wird hierbei explorativ vorgegangen, d.h. die zu Grunde liegende Faktorenstruktur wird nicht ex ante festgelegt. ${ }^{234}$ Zur Bestimmung der Anzahl der zu extrahierenden Faktoren wird auf das so genannte Kaiser-Kriterium zurückgegriffen. Demnach werden ausschließlich diejenigen Faktoren berücksichtigt, deren Eigenwert größer als eins

Vgl. Diamantopoulos/Winklhofer (2001), S. 271, Eberl (2004), S. 7 sowie Fassott/Eggert (2005), S. $38 \mathrm{f}$.

230 Vgl. hierzu die Ausführungen in Abschnitt E2.2 der vorliegenden Arbeit.

Zur Gütebeurteilung von formativen Messmodellen vgl. Diamantopoulos/Winklhofer (2001), S. $271 \mathrm{ff}$., Herrmann/Huber/Kressmann (2006), S. 45ff., Spillecke (2006), S. 94-97, Knollmann (2007), S. 111-115 sowie Diamantopoulos/Riefler/Roth (2008), S. 1215f.

Vgl. auch im Folgenden Homburg/Giering (1996), S. 8f. sowie Weber/Willauer/Schäffer (2003), S. 372.

233 Vgl. Homburg/Giering (1996), S. 8, Hüttner/Schwarting (2000), S. 383, Backhaus et al. (2008), S. 17 und 324 sowie Mayer (2009), S. 156.

$234 \mathrm{Vgl}$. Anderson/Gerbing (1988), S. 412 sowie Backhaus et al. (2008), S. 380f. 
ist. ${ }^{235}$ Der Eigenwert berechnet sich aus der Summe der quadrierten Faktorladungen ${ }^{236}$ eines Faktors über alle Indikatoren und dient als Maßstab für die durch den betrachteten Faktor erklärte Varianz der Beobachtungswerte. ${ }^{237}$ Anhand der Eindeutigkeit der Zuordnung von Indikatoren zu einem Faktor können im Zuge der explorativen Faktorenanalyse bereits erste Erkenntnisse bezüglich der Konvergenz- und Diskriminanzvalidität latenter Variablen gewonnen werden. So wird von Konvergenzvalidität ausgegangen, wenn die Indikatoren ausreichend hoch auf den extrahierten Faktor laden - in der Regel wird diese Anforderung ab einem Mindestfaktorladungswert von 0,4 als erfüllt betrachtet. Diskriminanzvalidität wird als gegeben angenommen, wenn die Faktorladungen ebendieser Indikatoren auf andere Faktoren signifikant niedriger ausfallen. ${ }^{238}$ Darüber hinaus kann die Güte der Messung eines Faktors anhand des durch ihn erklärten Anteils der Varianz der ihm zugehörigen Indikatoren beurteilt werden. Ein Faktor sollte mindestens 50\% der Varianz seiner Indikatoren erklären. ${ }^{239}$

Das Cronbach'sche Alpha misst die Reliabilität der Indikatoren eines Faktors und stellt somit ein Maß für die interne Konsistenz eines Faktors dar. ${ }^{240}$ Alpha, dessen Wertebereich sich von null bis eins erstreckt, wird umso größer, je höher die Kovarianzen bzw. Korrelationen zwischen den Indikatoren sind. ${ }^{241}$ Von einer akzeptablen Interne-Konsistenz-Reliabilität eines Faktors wird in der Regel ab einem Schwellenwert für das Cronbach'sche Alpha von 0,7 ausgegangen. ${ }^{242}$ Einschränkend gilt es diesbezüglich jedoch zu beachten, dass die Anzahl der Indikatoren eines Faktors das Cronbach'sche Alpha positiv beeinflusst. ${ }^{243}$ Somit kann es dazu kommen, dass sich bei einer großen Anzahl an Indikatoren ein hohes Alpha ergibt, obwohl keine zufriedenstellende interne Konsistenz vorliegt. ${ }^{244}$

Vgl. Hüttner/Schwarting (2000), S. 396, Backhaus et al. (2008), S. 353 sowie grundlegend Kaiser (1974).

Die Faktorladung ist eine Maßgröße für den Zusammenhang von Indikator und Faktor und stellt deren Korrelationskoeffizienten dar, vgl. Backhaus et al. (2008), S. 338.

Vgl. Backhaus et al. (2008), S. 353.

Vgl. Homburg/Giering (1996), S. 8.

Vgl. Homburg/Giering (1996), S. 12.

Vgl. Homburg/Giering (1996), S. 8 sowie grundlegend Cronbach (1951).

Vgl. Zinnbauer/Eberl (2004), S. 6.

Vgl. Nunnally (1978), S. 245 sowie Homburg/Giering (1996), S. 8.

Vgl. Homburg/Giering (1996), S. 8.

Vgl. Weber/Willauer/Schäffer (2003), S. 373. 
Das dritte Gütekriterium der ersten Generation, die Item to Total-Korrelation, wird im Gegensatz zu den ersten beiden Kriterien für jeden einzelnen der Indikatoren betrachtet. Die Item to Total-Korrelation eines Indikators ist die Korrelation dieses Indikators mit der Summe aller Indikatoren eines Faktors. ${ }^{245}$ Wird die Korrelation eines Indikators mit der Summe der übrigen Indikatoren ermittelt und somit der betrachtete Indikator nicht mit in die Summenbildung einbezogen, so ergibt sich die korrigierte Item to Total-Korrelation. ${ }^{246}$ Das Vorliegen hoher Item to TotalKorrelationen bei allen Indikatoren weist auf eine hohe Konvergenzvalidität hin. ${ }^{247}$ Die Item to Total-Korrelation wird zudem als Kriterium zur Eliminierung von Indikatoren im Falle einer zu geringen Interne-Konsistenz-Reliabilität empfohlen. Erreicht das Cronbach'sche Alpha nicht den geforderten Schwellenwert, so ist zur Steigerung der Reliabilität der Indikator mit der geringsten Item to Total-Korrelation zu eliminieren. ${ }^{248}$ Jeder Indikator sollte mindestens eine Item to Total-Korrelation von 0,5 aufweisen. ${ }^{249}$

Die Reliabilitäts- und Validitätskriterien der ersten Generation wurden vielfach kritisiert, da ihnen zum Teil sehr restriktive Annahmen zu Grunde liegen und folglich beispielsweise keine explizite Schätzung von Messfehlern möglich ist. Des Weiteren basiert die Validitätsbeurteilung nicht auf inferenzstatistischen Prüfungen von Modellparametern, sondern weitestgehend auf Faustregeln. ${ }^{250}$ Die Gütekriterien der zweiten Generation beheben diese Defizite. Sie werden daher in der vorliegenden Arbeit zusätzlich zu den Kriterien der ersten Generation verwendet und nachfolgend dargestellt.

Die Reliabilitäts- und Validitätskriterien der zweiten Generation basieren auf der maßgeblich von Jöreskog entwickelten konfirmatorischen Faktorenanalyse. ${ }^{251}$ Diese unterscheidet sich von der explorativen Faktorenanalyse insbesondere darin, dass a priori - d.h. im Vorfeld der Untersuchung - auf Basis theoretischer Vorüberlegungen

Vgl. Homburg/Giering (1996), S. 8.

Vgl. Janssen/Laatz (2007), S. 598. Aufgrund der höheren Aussagekraft wird in der vorliegenden Arbeit ausschließlich auf die korrigierte Item to Total-Korrelation zurückgegriffen, der Zusatz 'korrigierte' jedoch weggelassen.

Vgl. Weber/Willauer/Schäffer (2003), S. 373.

Vgl. Churchill (1979), S. 68 sowie Janssen/Laatz (2007), S. 598.

Vgl. Bearden/Netemeyer/Teel (1989), S. 473.

Vgl. Homburg/Giering (1996), S. 9 m.w.N.

Vgl. grundlegend Jöreskog (1966), Jöreskog (1967) sowie Jöreskog (1969). 
Hypothesen über die Zuordnung der einzelnen beobachtbaren Indikatoren $\mathrm{zu}$ den dahinter stehenden, nicht beobachtbaren Faktoren aufgestellt werden. Die so postulierte Faktorenstruktur wird dann im Rahmen der empirischen Analyse überprüft. ${ }^{252}$ Die konfirmatorische Faktorenanalyse entspricht einem Spezialfall des allgemeinen Modells der im nachfolgenden Abschnitt C3.5 näher erläuterten Kovarianzstrukturanalyse und stellt deren Messmodell dar. ${ }^{253}$ Ziel der Analyse ist es, die Modellparameter derart zu schätzen, dass die aus der Modellspezifizierung resultierende Kovarianzmatrix die empirische Kovarianzmatrix möglichst gut widerspiegelt. ${ }^{254}$

Voraussetzung für diese Parameterschätzung ist die Identifizierung des spezifizierten Modells. Hierzu müssen in der empirischen Kovarianzmatrix genügend Informationen für eine eindeutige Schätzung der Modellparameter enthalten sein. Als notwendige Bedingung gilt in diesem Zusammenhang, dass die Anzahl der zu schätzenden Modellparameter maximal so hoch sein darf wie die Anzahl der empirischen Varianzen und Kovarianzen. ${ }^{255}$ Im Falle einer einfaktoriellen konfirmatorischen Faktorenanalyse sind somit mindestens drei Indikatoren erforderlich. Die Anzahl der Freiheitsgrade - also die Differenz aus der Anzahl der empirischen Varianzen und Kovarianzen und der Anzahl der zu schätzenden Parameter - beträgt hier folglich null. ${ }^{256}$

Im Anschluss an die Parameterschätzung erfolgt die Gütebeurteilung des spezifizierten Modells anhand von verschiedenen globalen und lokalen Gütekriterien. ${ }^{257}$ Während globale Gütekriterien die Anpassungsgüte des gesamten Modells an die empirischen Daten überprüfen, beziehen sich lokale Gütekriterien lediglich auf einzelne Modellbestandteile.

In der vorliegenden Arbeit werden als globale Gütekriterien der Chi-QuadratAnpassungstest ( $\chi^{2}$-Anpassungstest), der Root Mean Square Error of Approximation

Vgl. Homburg/Giering (1996), S. 9, Reinecke (2005), S. 134f. sowie Backhaus et al. (2008), S. 381.

Vgl. Homburg/Giering (1996), S. 9, Reinecke (2005), S. 136 sowie Backhaus et al. (2008), S. 519-521.

Vgl. Homburg/Baumgartner (1995a), S. 165, Homburg/Baumgartner (1995b), S. 1093, Homburg/Giering (1996), S. 9 sowie Homburg/Pflesser (2000a), S. 425.

Vgl. Homburg (1992a), S. 502f., Homburg/Pflesser (2000b), S. 645 sowie Reinecke (2005), S. 139.

Vgl. Reinecke (2005), S. 139f.

Vgl. auch im Folgenden Homburg/Baumgartner (1995a), S. 165, Homburg/Giering (1996),

S. 9f., Homburg/Pflesser (2000a), S. 426 sowie Homburg/Pflesser (2000b), S. 646. 
(RMSEA), der Standardized Root Mean Square Residual (SRMR), der Goodness of Fit Index (GFI), der Adjusted Goodness of Fit Index (AGFI), der Comparative Fit Index (CFI) sowie der Tucker Lewis Index (TLI) verwendet.

Der $\chi^{2}$-Anpassungstest ist ein inferenzstatistisches Anpassungsmaß, das die absolute 'Richtigkeit' des spezifizierten Modells prüft. Die dem Test zu Grunde liegende Nullhypothese besagt, dass die aus dem spezifizierten Modell resultierende Kovarianzmatrix exakt mit der empirischen Kovarianzmatrix übereinstimmt und das spezifizierte Modell somit 'richtig' ist. ${ }^{258}$ Für den zur Beurteilung des $\chi^{2}$-Wertes herangezogenen p-Wert wird ein Mindestniveau von 0,05 gefordert. Bei Überschreitung dieses Wertes kann die Richtigkeit des Modells auf einem Signifikanzniveau von 5\% nicht abgelehnt werden. ${ }^{259}$ Der $\chi^{2}$-Anpassungstest unterliegt allerdings einigen gravierenden Restriktionen, die seine Nützlichkeit einschränken. Kritisiert wird insbesondere, dass Modelle niemals vollkommen zutreffend sind und eine Überprüfung auf absolute Richtigkeit demnach wenig sinnvoll ist. ${ }^{260}$ Darüber hinaus wird bei ausreichend groBem Stichprobenumfang nahezu jedes Modell abgelehnt, da der $\chi^{2}$-Wert proportional zum Stichprobenumfang steigt. ${ }^{261}$ Daher wird empfohlen, den $\chi^{2}$-Wert als deskriptives Gütekriterium zu verwenden, indem dieser durch die Anzahl der Freiheitsgrade dividiert wird. ${ }^{262}$ Für eine gute Modellanpassung sollte der sich hieraus ergebende Wert nicht größer als zwei sein. ${ }^{263}$

Als weiteres globales, inferenzstatistisches Maß fungiert der Root Mean Square Error of Approximation (RMSEA). Dieser untersucht im Gegensatz zum $\chi^{2}$ Anpassungstest nicht die absolute Richtigkeit eines Modells, sondern ob ein Modell die Realität gut approximiert. ${ }^{264}$ Hierbei wird die Modellkomplexität berücksichtigt, indem die Anzahl der Freiheitsgrade die Höhe des RMSEA negativ beeinflusst. Ein komplexes Modell - dieses geht mit einer geringeren Anzahl an Freiheitsgraden

Vgl. Homburg/Baumgartner (1995a), S. 166, Homburg/Giering (1996), S. 10 sowie Byrne (2001), S. 79.

Vgl. Homburg/Baumgartner (1995a), S. 166 sowie Homburg/Giering (1996), S. 10.

Vgl. Bagozzi (1982), S. 327.

Vgl. Homburg/Baumgartner (1995a), S. 170 sowie Homburg/Giering (1996), S. 10.

Vgl. Byrne (1989), S. 55.

Vgl. Homburg/Baumgartner (1995a), S. 166 sowie grundlegend Steiger (1990), hier insbesondere S. $176 f$. 
einher - weist folglich einen vergleichsweise höheren RMSEA auf. ${ }^{265}$ Werte, die kleiner als 0,05 sind, deuten auf eine gute Modellanpassung hin. ${ }^{266}$

Der Standardized Root Mean Square Residual (SRMR) ist ein globales, deskriptives $\mathrm{Ma} ß$ und kennzeichnet die durchschnittliche Größe der Residuen zwischen den Elementen der empirischen und der vom Modell reproduzierten Korrelationsmatrix. ${ }^{267}$ Der Wertebereich des SRMR ist auf das Intervall zwischen null und eins normiert, wobei ein Wert von null einen perfekten Modellfit anzeigt. ${ }^{268}$ Von einer guten Modellanpassung wird in der Regel bei Werten bis 0,05 ausgegangen. ${ }^{269}$

Der Goodness of Fit Index (GFI) und der Adjusted Goodness of Fit Index (AGFI) stellen ebenfalls globale, deskriptive Gütekriterien dar. ${ }^{270}$ Beide messen - ähnlich wie das Bestimmtheitsmaß im Rahmen der Regressionsanalyse - den Anteil von Varianz und Kovarianz, den das Modell zu erklären in der Lage ist. ${ }^{271}$ Die Aussagekraft des GFI ist jedoch dadurch eingeschränkt, dass sich das Hinzufügen eines Modellparameters automatisch positiv oder allenfalls neutral auf die Beurteilung der Anpassungsgüte auswirkt. Der resultierende Verlust an Freiheitsgraden wird nicht negativ bewertet. ${ }^{272}$ Diesen Schwachpunkt kann der AGFI ausgleichen, da er die Modellkomplexität in Form eines von der Anzahl der Freiheitsgrade abhängigen Strafterms berücksichtigt. Somit werden bei identischer Anpassungsgüte sparsame Modelle mit weniger Parametern besser beurteilt als Modelle, die mehr Parameter benötigen. ${ }^{273}$ Der Wertebereich von GFI und AGFI liegt zwischen null und eins. Für beide Gütekriterien gilt, dass der

Vgl. Bühner (2006), S. 255f.

Vgl. Browne/Cudeck (1993), S. 144.

Vgl. Byrne (2001), S. 82 sowie Schermelleh-Engel/Moosbrugger/Müller (2003), S. 38. Der SRMR wurde von Bentler eingeführt und stellt eine standardisierte Version des von Jöreskog und Sörbom entwickelten Root Mean Square Residual (RMR) dar, vgl. grundlegend Bentler (1995) bzw. Jöreskog/Sörbom (1981).

Durch den Rückgriff auf die Korrelationsmatrizen und die damit einhergehende Standardisierung ist der SRMR gut interpretierbar. Hingegen ist auf Basis des RMR keine verlässliche Aussage hinsichtlich des Modellfits moglich, da die hier betrachteten Residuen zwischen der empirischen und der aus dem Modell abgeleiteten Kovarianzmatrix von den zu Grunde gelegten Skalierungen der gemessenen Variablen abhängig sind. Vgl. Byrne (2001), S. 82, Schermelleh-Engel Moosbrugger/Müller (2003), S. 38 sowie Reinecke (2005), S. $122 \mathrm{f}$.

Vgl. Byrne (2001), S. 82 sowie Schermelleh-Engel/Moosbrugger/Müller (2003), S. 38.

Vgl. Homburg/Giering (1996), S. 10 sowie Homburg/Pflesser (2000a), S. 427.

Vgl. Backhaus et al. (2006), S. 380. Da die hier zitierte Textstelle in der uberarbeiteten Druckversion der 12. Auflage nicht mehr enthalten ist, wird ausnahmsweise auf die 11. Auflage verwiesen.

Vgl. Homburg/Baumgartner (1995a), S. 166 sowie Homburg/Giering (1996), S. 10.

Vgl. Homburg/Baumgartner (1995a), S. 166 und 170 sowie Homburg/Giering (1996), S. 10. 
Modellfit umso besser ist, je näher sich der Wert an eins annähert. ${ }^{274}$ Eine hohe Anpassungsgüte wird jeweils bei Werten ab 0,9 unterstellt. ${ }^{275}$

Bei dem Comparative Fit Index (CFI) handelt es sich um ein inkrementelles Anpassungsmaß ${ }^{276}$ Diese Maße sind grundsätzlich dadurch charakterisiert, dass sie die Verbesserung der Anpassungsgüte im Zuge des Übergangs von einem Basismodell zum relevanten Modell analysieren. Das Basismodell (auch: Nullmodell) unterstellt hierbei, dass sämtliche Indikatorvariablen unkorreliert sind, und besitzt folglich keinerlei inhaltliche Plausibilität. ${ }^{277}$ Zur Berechnung des CFI werden die jeweils um die Anzahl an Freiheitsgraden reduzierten $\chi^{2}$-Werte des zu beurteilenden Modells zu den in gleicher Weise bereinigten $\chi^{2}$-Werten des Basismodells in Relation gesetzt. ${ }^{278}$ Der CFI kann Werte zwischen null und eins annehmen, wobei höhere Werte eine bessere Anpassungsgüte anzeigen. Ein guter Modellfit wird bei Werten ab 0,97 angenommen. $^{279}$

Der Tucker Lewis Index (TLI) stellt ebenfalls ein globales, inkrementelles Gütekriterium dar. ${ }^{280}$ Bei der Berechnung des TLI werden alle $\chi^{2}$-Werte mit ihren jeweiligen Freiheitsgraden in Beziehung gesetzt. ${ }^{281}$ Der TLI ist weitestgehend unabhängig von der Stichprobengröße und nimmt normalerweise Werte zwischen null und eins an. ${ }^{282}$ Analog zum CFI wird im Falle des TLI bei Werten ab 0,97 von einer hohen Anpassungsgüte ausgegangen. ${ }^{283}$

Vgl. Bollen (1989), S. 276, Homburg/Giering (1996), S. 10 sowie Homburg/Pflesser (2000a), S. 428 .

Vgl. Bagozzi/Yi (1988), S. 82 sowie Homburg/Baumgartner (1995a), S. 167 f.

Vgl. Homburg/Pflesser (2000a), S. 426f. sowie grundlegend Bentler (1990).

Vgl. Homburg/Baumgartner (1995a), S. 170, Homburg/Pflesser (2000a) S. 426, Zinnbauer/Eberl (2004), S. 11f. sowie Reinecke (2005), S. $125 \mathrm{f}$.

Vgl. Homburg/Baumgartner (1995a), S. 168, Homburg/Pflesser (2000a) S. 427 sowie Schermelleh-Engel/Moosbrugger/Müller (2003), S. $41 \mathrm{f}$.

Vgl. Schermelleh-Engel/Moosbrugger/Müller (2003), S. 42.

Vgl. Reinecke (2005), S. 126f. sowie grundlegend Tucker/Lewis (1973). Der TLI wird teilweise auch als Non-Normed Fit Index (NNFI) bezeichnet, vgl. Bentler (1990), S. 239 sowie Schermelleh-Engel/Moosbrugger/Müller (2003), S. $40 \mathrm{f}$.

Vgl. Homburg/Baumgartner (1995a), S. 168 sowie Reinecke (2005), S. 127.

Ein Wert von eins zeigt hierbei einen optimalen Modellfit an. Allerdings kann der TLI auch Werte größer eins annehmen. Ein solches Ergebnis deutet auf ein so genanntes 'overfitting' hin, d.h. im Modell sind mehr Parameter als notwendig spezifiziert. Vgl. Bollen (1989), S. 273 sowie Schermelleh-Engel/Moosbrugger/Müller (2003), S. 41.

Vgl. Schermelleh-Engel/Moosbrugger/Müller (2003), S. 42. 
Neben den dargestellten globalen Gütekriterien werden in der vorliegenden Arbeit zur Beurteilung von Modellteilstrukturen die lokalen Gütekriterien Indikatorreliabilität, $\mathrm{t}$-Wert der Faktorladung, Faktorreliabilität und durchschnittlich erfasste Varianz (DEV) eingesetzt. Während sich die beiden erstgenannten Kriterien auf einzelne Indikatoren beziehen, erfolgt die Anwendung der beiden letztgenannten Kriterien für ganze Faktoren. ${ }^{284}$

Die Indikatorreliabilität ist allgemein definiert als quadrierte Korrelation zwischen einem Konstrukt und einem der zugehörigen Indikatoren. Sie misst den Anteil der Varianz des Indikators, der durch den zu Grunde liegenden Faktor erklärt wird. Der nicht durch den Faktor erklärte Varianzanteil entfällt hingegen auf die Messfehlervariable des Indikators. In der Regel wird für die Indikatorreliabilität ein Mindestwert von 0,4 verlangt. ${ }^{285}$

Neben der Betrachtung der Indikatorreliabilität wird ergänzend getestet, ob sich die Faktorladung eines Indikators signifikant von null unterscheidet. Dies ist bei einem Signifikanzniveau von 5\% dann der Fall, wenn der $\mathrm{t}$-Wert der Faktorladung mindestens 1,645 beträgt (einseitiger Test). ${ }^{286}$ Der t-Wert der Faktorladung - auch bezeichnet als Critical Ratio (C.R.) - entspricht dem Quotienten aus unstandardisierter Faktorladung und Standardfehler der Schätzung. ${ }^{287}$

Zur Beurteilung der Frage, wie gut ein Faktor durch die Gesamtheit der ihm zu Grunde liegenden Indikatoren abgebildet wird, können die Gütekriterien Faktorreliabilität und durchschnittlich erfasste Varianz (DEV) herangezogen werden. Der Wertebereich liegt für beide Größen zwischen null und eins, wobei hohe Werte auf eine hohe Qualität der Messung hinweisen. ${ }^{288}$ Für die Faktorreliabilität wird ein Schwellenwert von 0,6 gefordert, während die durchschnittlich erfasste Varianz mindestens 0,5 betragen sollte. ${ }^{289}$

Vgl. Homburg/Pflesser (2000a), S. 428.

Vgl. Homburg/Baumgartner (1995a), S. 170 sowie Homburg/Giering (1996), S. 10.

Vgl. Homburg/Giering (1996), S. 11.

Vgl. Backhaus et al. (2006), S. 383. Da die hier zitierte Textstelle in der überarbeiteten Druckversion der 12. Auflage nicht mehr enthalten ist, wird ausnahmsweise auf die 11. Auflage verwiesen.

Vgl. Homburg/Baumgartner (1995a), S. 170 sowie Homburg/Giering (1996), S. $10 \mathrm{f}$.

Vgl. Bagozzi/Yi (1988), S. 82 sowie Homburg/Baumgartner (1995a), S. 170. 
Die zur Beurteilung der reflektiven Messmodelle in der vorliegenden Arbeit verwendeten Gütekriterien der ersten und zweiten Generation sowie das jeweils zu Grunde gelegte Anspruchsniveau sind in Tabelle 6 zusammenfassend dargestellt.

\begin{tabular}{|l|c|}
\hline Gütekriterien der ersten Generation & \multicolumn{1}{|c|}{ Anspruchsniveau } \\
\hline $\begin{array}{l}\text { Erklärte Varianz im Rahmen der explorativen } \\
\text { Faktorenanalyse }\end{array}$ & $\geq 0,5$ \\
\hline Cronbach'sches Alpha & $\geq 0,7$ \\
\hline Item to Total-Korrelation & $\begin{array}{l}\text { Wenn Cronbach'sches Alpha kleiner 0,7: } \\
\text { Eliminierung des Indikators mit der niedrigsten } \\
\text { Item to Total-Korrelation }\end{array}$ \\
\hline Gütekriterien der zweiten Generation & \multicolumn{1}{c|}{ Anspruchsniveau } \\
\hline$\chi^{2} /$ df & $\leq 2,0$ \\
\hline RMSEA & $\leq 0,05$ \\
\hline SRMR & $\leq 0,05$ \\
\hline GFI & $\geq 0,9$ \\
\hline AGFI & $\geq 0,9$ \\
\hline CFI & $\geq 0,97$ \\
\hline TLI & $\geq 0,97$ \\
\hline Indikatorreliabilität & $\geq 0,4$ \\
\hline t-Wert der Faktorladung & $\geq 1,645$ \\
\hline Faktorreliabilität & $\geq 0,6$ \\
\hline Durchschnittlich erfasste Varianz (DEV) & $\geq 0,5$ \\
\hline
\end{tabular}

Tabelle 6: Gütekriterien zur Beurteilung der reflektiven Messmodelle

Die dargestellten Gütekriterien dienen überwiegend der Beurteilung der Reliabilität und Konvergenzvalidität von Messmodellen. Ein weiterer wichtiger Aspekt, der einer Überprüfung bedarf, ist die Diskriminanzvalidität. Sie bezieht sich auf mehrere Faktoren und stellt sicher, dass diese nicht den gleichen, sondern unterschiedliche Sachverhalte abbilden. ${ }^{290}$ Für die Analyse existieren mit dem $\chi^{2}$-Differenztest und dem Fornell/Larcker-Kriterium zwei alternative Optionen. ${ }^{291} \mathrm{Da}$ es als das wesentlich strengere der beiden Verfahren gilt, wird in der vorliegenden Arbeit ausschließlich das

290 Vgl. hierzu die obigen Ausfuhrungen zur Diskriminanzvaliditat sowie Homburg/Giering (1996), S. 7 und Homburg (2007), S. 47.

291 Vgl. auch im Folgenden Homburg/Giering (1996), S. 11 sowie Homburg/Pflesser (2000a), S. 429. 
Fornell/Larcker-Kriterium verwendet. Es verlangt, dass die durchschnittlich erfasste Varianz eines Faktors größer ist als die quadrierte Korrelation dieses Faktors mit sämtlichen übrigen Faktoren. ${ }^{292}$ Ist diese Anforderung erfüllt, wird von Diskriminanzvalidität zwischen den einzelnen Faktoren ausgegangen.

\subsection{Kovarianzstrukturanalyse}

Die Kovarianzstrukturanalyse ${ }^{293}$ zählt zu den Strukturen-prüfenden Verfahren und dient der Untersuchung von Abhängigkeitsbeziehungen zwischen Variablen. ${ }^{294} \mathrm{Die}$ Grundlage hierfür bilden die empirisch gemessenen Varianzen und Kovarianzen der Indikatorvariablen. ${ }^{295}$ Aus methodischer Sicht stellt die Kovarianzstrukturanalyse eine Kombination aus konfirmatorischer Faktorenanalyse und Pfadanalyse dar und gilt als das derzeit leistungsfähigste multivariate Analyseverfahren. ${ }^{296}$ Die Überlegenheit der Kovarianzstrukturanalyse gegenüber traditionellen Methoden, wie z.B. der multiplen Regressionsanalyse, liegt insbesondere darin begründet, dass

- sowohl manifeste (d.h. direkt messbare) als auch latente Variablen in die Untersuchung einfließen können,

- Messfehler der Indikatorvariablen bei der Abbildung von latenten Variablen explizit berücksichtigt werden,

Vgl. Fornell/Larcker (1981), S. 46.

In der Literatur wird die Kovarianzstrukturanalyse mitunter auch als Strukturgleichungsanalyse, SEM (Structural Equation Modeling) bzw. LISREL (Linear Structural Relations)-Ansatz sowie dies ist der am hăufigsten verwendete Begriff - als Kausalanalyse bezeichnet. Der Begriff Kausalanalyse ist jedoch irreführend, da er suggeriert, dass das Verfahren die Analyse von Kausalitäten ermöglicht. Dies ist aus wissenschaftstheoretischer Sicht jedoch nicht auf Basis der hier zu Grunde gelegten Analyse der Kovarianzstrukturen von Querschnittdaten, sondern ausschließlich mittels kontrollierter Experimente möglich. Vgl. Homburg/Hildebrandt (1998), S. 17, Homburg/ Pflesser (2000b), S. 635, Reinecke (2005), S. 12, Scholderer/Balderjahn/Paulssen (2006), S. 641 sowie Schnell/Hill/Esser (2008), S. 463. Daher wird in der vorliegenden Arbeit auf den präziseren Begriff der Kovarianzstrukturanalyse zuruckgegriffen.

Vgl. Backhaus et al. (2008), S. 11.

Vgl. Homburg/Pflesser (2000b), S. 635.

Vgl. Hildebrandt (1984), S. 44, Homburg (1992a), S. 499 sowie Reinecke (2005), S. 9. In jüngster Zeit wird mit dem Partial Least Squares (PLS)-Ansatz wieder vermehrt auf ein varianzbasiertes Strukturgleichungsverfahren als Alternative zur Kovarianzstrukturanalyse zurückgegriffen. Zu einem ausfuhrlichen Vergleich der beiden Ansătze vgl. Herrmann/Huber/Kressmann (2006) sowie Scholderer/Balderjahn (2006). Diese Entwicklung wird jedoch überwiegend kritisch betrachtet, wie das Editorial von Balderjahn (2008) exemplarisch zeigt. Scholderer/Balderjahn (2006) fassen diesbezüglich zusammen (S. 67): „LISREL [weist] das deutlich höhere Leistungsund Anwendungspotenzial auf. PLS sollte [...] insofern nur eingesetzt werden, wenn der Einsatz von LISREL definitiv nicht möglich ist.“ 
- komplexe Dependenzstrukturen inklusive indirekter Effekte modelliert und simultan geschätzt werden können sowie

- die aufgestellten Hypothesen bzw. das sich daraus ergebende Modell anhand von Teststatistiken und Gütekriterien überprüft werden können. ${ }^{297}$

Daher wird die Kovarianzstrukturanalyse zur Untersuchung der im Rahmen der dritten Forschungsfrage im Mittelpunkt stehenden Abhängigkeitsbeziehung der Integration des Rechungswesens, der Einheitlichkeit der Finanzsprache und des Erfolgs der Controllerarbeit eingesetzt.

Die Spezifikation eines vollständigen zu untersuchenden Modells umfasst mindestens zwei Messmodelle und ein Strukturmodell. Diese lassen sich in der Vektorschreibweise der LISREL-Notation folgendermaßen ausdrücken: ${ }^{298}$

$$
\begin{aligned}
& \eta=B \eta+\Gamma \xi+\zeta \\
& \mathbf{y}=\Lambda_{\mathbf{y}} \eta+\varepsilon \\
& \mathbf{x}=\Lambda_{\mathbf{x}} \xi+\delta
\end{aligned}
$$

Hierbei stellt Gleichung (1) das Strukturmodell dar, welches auf Basis theoretischer Überlegungen die hypothetischen Beziehungen zwischen den endogenen latenten Variablen (mit $\eta$ bezeichnet) und den exogenen latenten Variablen (mit $\xi$ bezeichnet) abbildet. Die Koeffizientenmatrix B bildet die Effekte zwischen latenten endogenen Variablen $a b$, während die Koeffizientenmatrix $\Gamma$ die Effekte latenter exogener auf latente endogene Variablen modelliert. Der Vektor $\zeta$ erfasst Fehlergrößen im Strukturmodell und entspricht dem Fehlerterm im Rahmen der multiplen Regressionsanalyse.

Die Gleichungen (2) und (3) stellen faktorenanalytische Modelle dar, die auch als Messmodelle bezeichnet werden. Sie bilden die Beziehung zwischen den Konstrukten

Vgl. Gregson (1992), Homburg/Pflesser (2000b), S. 635f., Byrne (2001), S. 3f. sowie Smith/Langfield-Smith (2004), S. 59f. Homburg und Klarmann bringen die Leistungsstärke der Kovarianzstrukturanalyse anschaulich zum Ausdruck: „Als erste und bisher einzige Methode gibt sie eine Antwort auf zwei zentrale Probleme wissenschaftlichen Arbeitens: die Analyse von Dependenzen und die Messung komplexer Konstrukte" (Homburg/Klarmann (2006), S. 728).

Vgl. auch zu den folgenden Ausführungen Jöreskog/Sörbom (1982), S. 404f., Bollen (1989), S. 319f., Bagozzi/Baumgartner (1994), S. 386f., Homburg/Baumgartner (1995a), S. 163 sowie Homburg/Pflesser (2000b), S. 640f. 
( $\eta$ bzw. $\xi$ ) und den jeweils zugehörigen Indikatoren ab. Der Vektor y enthält hierbei die Indikatoren der latenten endogenen Variablen, der Vektor $\mathrm{x}$ die der latenten exogenen Variablen. Die Koeffizientenmatrizen $\Lambda_{\mathrm{y}}$ und $\Lambda_{\mathrm{x}}$ können als Faktorladungsmatrizen interpretiert werden, während die Vektoren $\varepsilon$ und $\delta$ Messfehlervariablen enthalten. Letztere bringen zum Ausdruck, dass jeder Indikator als eine fehlerbehaftete Messung einer - unter Umständen auch mehrerer - latenten Variablen betrachtet wird.

Abbildung 11 fasst die verschiedenen Bestandteile eines Kovarianzstrukturmodells unter Verwendung der LISREL-Notation beispielhaft zusammen.

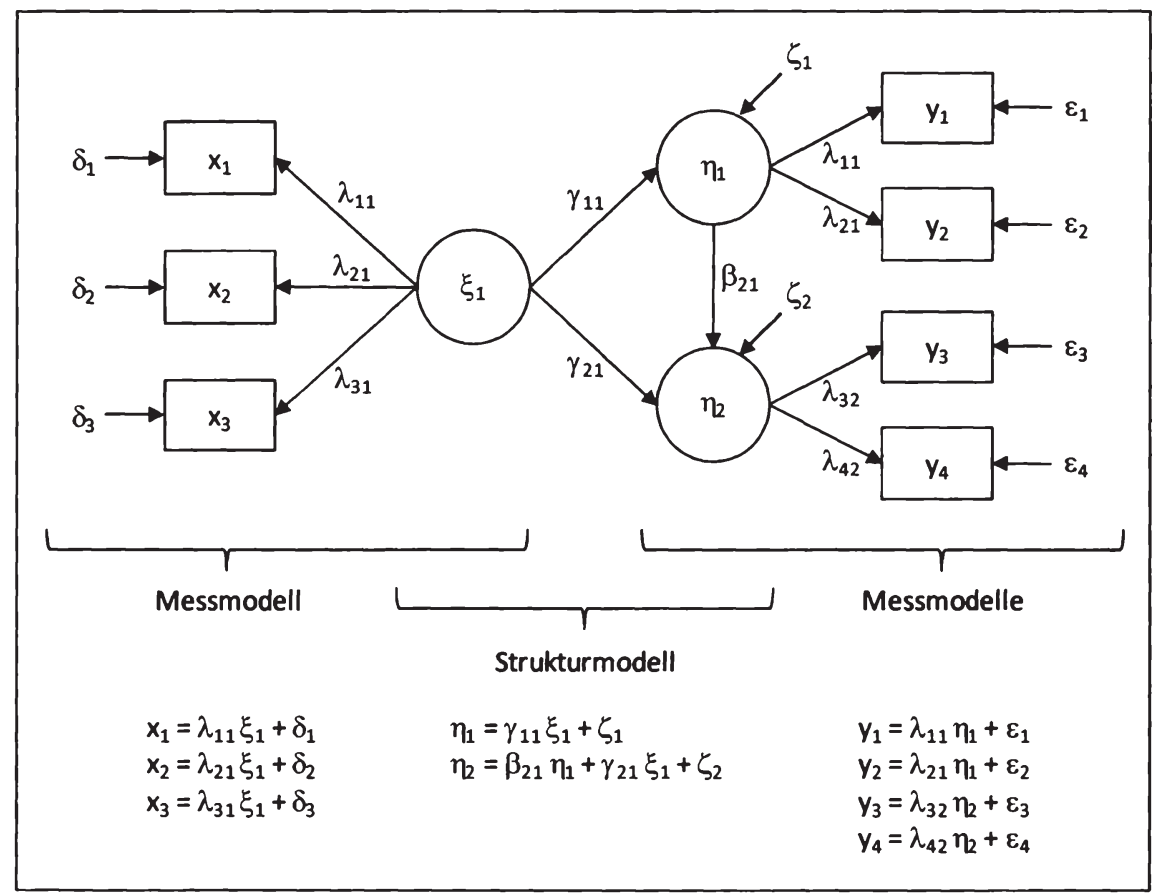

Abbildung 11: Beispielhaftes Kovarianzstrukturmodell ${ }^{299}$ 
Unter geeigneten Voraussetzungen ${ }^{300}$ kann die Kovarianzmatrix $\Sigma$ der beobachteten Indikatorvariablen $\mathrm{y}$ und $\mathrm{x}$ durch acht Parametermatrizen folgendermaßen ausgedrückt werden:

$$
\Sigma=\Sigma\left(\mathrm{B}, \Gamma, \Lambda_{\mathrm{y}}, \Lambda_{\mathrm{x}}, \Phi, \Psi, \Theta_{\varepsilon}, \Theta_{\delta}\right)
$$

Hierbei bezeichnen die vier zuletzt genannten Parametermatrizen die Kovarianzmatrizen der Vektoren $\xi, \zeta, \varepsilon$ und $\delta$. Bezeichnet man die Gesamtheit der zu schätzenden Parameter innerhalb der acht Parametermatrizen mit $\alpha$, so lässt sich Gleichung (4) vereinfacht darstellen als

$$
\Sigma=\Sigma(\alpha)
$$

Die Kovarianzmatrix der Indikatorvariablen wird somit als Funktion des Vektors der zu schätzenden Parameter ausgedrückt. ${ }^{301}$

Die anschließende Parameterschätzung verfolgt das Ziel, einen Vektor $\widehat{\alpha}$ so zu ermitteln, dass die auf Basis des Modells generierte Kovarianzmatrix $\widehat{\Sigma}=\Sigma(\widehat{\alpha})$ die empirisch ermittelte Kovarianzmatrix $S$ möglichst exakt reproduziert. Dies wird erreicht durch die Lösung des Minimierungsproblems

$$
\mathrm{f}_{\mathrm{S}}(\alpha)=\mathrm{F}(\mathrm{S}, \Sigma(\alpha)) \rightarrow \min
$$

wobei $\mathrm{F}$ die Diskrepanzfunktion kennzeichnet, die die Unterschiedlichkeit zweier symmetrischer Matrizen misst.

Eine Grundvoraussetzung für die Durchführung der Kovarianzstrukturanalyse stellt die Identifikation des spezifizierten Modells dar, d.h. die Kovarianzmatrix der Indikatorvariablen muss genügend Informationen für eine eindeutige Schätzung der Modellparameter enthalten. Als notwendige Bedingung für die Identifizierung eines Modells mit q Indikatoren gilt: ${ }^{302}$

$$
\mathrm{t} \leq \frac{\mathrm{q} \cdot(\mathrm{q}+1)}{2}
$$

\footnotetext{
300 Vgl. hierzu im Detail Homburg (1989), S. $151 \mathrm{ff}$.

301

Vgl. auch zur nachfolgenden Formel (7) sowie den zugehörigen Erläuterungen Bagozzi/Baumgartner (1994), S. 387, Homburg/Baumgartner (1995a), S. 164f., Homburg/Hildebrandt (1998), S. 21 f. sowie Homburg/Pflesser (2000b), S. 643f.

Vgl. auch im Folgenden Bollen (1989), S. 326ff., Homburg (1992a), S. 502f., Homburg/ Baumgartner (1995b), S. 1093 sowie Homburg/Pflesser (2000b), S. 645.
} 
Demnach darf die Anzahl der zu schätzenden Parameter $(t)$ maximal so groß sein wie die Anzahl der empirischen Varianzen und Kovarianzen $(\mathrm{q} \cdot(\mathrm{q}+1) / 2)$. Letztere ist identisch mit der Anzahl an Gleichungen, die das Kovarianzstrukturmodell umfasst. Ein eindeutiger Rückschluss auf die Modellparameter ist folglich unmöglich, wenn die Anzahl der Modellparameter höher ist als die Anzahl der zur Verfügung stehenden Gleichungen. Die Differenz aus der Anzahl der Modellparameter und der Anzahl der Gleichungen ergibt die Freiheitsgrade eines Modells. Trotz Erfüllung der notwendigen Bedingung aus Gleichung (7) ist bislang keine hinreichende Bedingung fur die Identifikation eines Modells bekannt, sodass diesbezïglich niemals endgültige Sicherheit besteht. Als Anzeichen für nicht identifizierte Modelle gelten große Standardfehler sowie unverständliche oder entartete Schätzer, wie z.B. negative Fehlervarianzen.

Im Anschluss an die Parameterschätzung erfolgt die Beurteilung der Anpassungsgüte des Modells bzw. der Modellkomponenten anhand der bereits in Abschnitt C3.4 vorgestellten globalen und lokalen Gütekriterien. Ergeben sich diesbezüglich zufriedenstellende Ergebnisse, so kann zur inhaltlichen Interpretation der zwischen den latenten Variablen bestehenden Abhängigkeitsbeziehungen übergegangen werden. ${ }^{303}$ Eine besondere Bedeutung kommt hierbei der quadrierten multiplen Korrelation der endogenen latenten Variablen als lokales Gütemaß des Strukturmodells zu. Diese auch als $R^{2}$ bezeichnete Größe gibt an, welcher Anteil der Varianz einer latenten endogenen Variablen durch diejenigen latenten Variablen erklärt wird, die gemäß der Modellspezifizierung einen Effekt auf sie ausüben. ${ }^{304}$ Der Wertebereich der quadrierten multiplen Korrelation liegt zwischen null und eins, wobei der verbleibende Anteil der Varianz einer endogenen latenten Variablen auf deren Fehlervariable entfällt. Kleine $\mathrm{R}^{2}$-Werte deuten somit darauf hin, dass nicht im Modell enthaltene Größen einen wesentlichen Einfluss auf die betrachtete endogene latente Variable ausüben.

Neben der quadrierten multiplen Korrelation bilden die standardisierten Effekte des Strukturmodells ( $\beta$ - und $\gamma$-Koeffizienten) ${ }^{305}$ sowie deren jeweils korrespondierende t-Werte einen wichtigen Ansatz zur Überprüfung einer hypothetischen Dependenzstruktur. Während ein standardisierter Effekt Aussagen hinsichtlich der Stärke und des

Vgl. Homburg (1992a), S. 506 sowie Homburg/Hildebrandt (1998), S. 26.

Vgl. auch im Folgenden Hildebrandt (1984), S. 49, Homburg/Baumgartner (1995a), S. 170f., Homburg/Pflesser (2000b), S. 649 sowie Byrne (2001), S. 163.

Vgl. hierzu Gleichung (1) sowie Abbildung 11 der vorliegenden Arbeit. 
Vorzeichens einer Dependenzbeziehung ermöglicht - der Wertebereich ist hierbei auf minus eins bis plus eins normiert -, gibt der t-Wert Auskunft über deren Signifikanz und bildet somit die Grundlage für die Annahme oder Ablehnung der zu untersuchenden Hypothese. ${ }^{306}$

Weder für die quadrierte multiple Korrelation noch für die standardisierten Effekte werden Mindestwerte verlangt, da die Beurteilung von der jeweiligen Fragestellung abhängt. Für die t-Werte werden die üblichen Grenzwerte der t-Teststatistik herangezogen. $^{307}$

Von hoher Bedeutung für die im Rahmen der dritten Forschungsfrage durchgeführte Analyse der Dependenzbeziehungen der Integrationsintensität des Rechnungswesens, der Einheitlichkeit der Finanzsprache sowie des Erfolgs der Controllerarbeit ist darüber hinaus zum einen die Effektzerlegung in direkte, indirekte und Gesamteffekte sowie zum anderen die Untersuchung moderierender Effekte.

Ein indirekter (auch: mediierender) Effekt liegt vor, wenn eine Variable X über eine Variable $\mathrm{M}$ auf eine Variable $\mathrm{Y}$ wirkt, d.h. es besteht ein direkter Effekt von $\mathrm{X}$ auf $M$ und von $M$ auf $Y$. In diesem Fall stellt $M$ die mediierende Variable dar. Es lassen sich zwei Formen der Mediation unterscheiden: Vollständige Mediation liegt vor, wenn nach Kontrolle des indirekten Effektes von X über $M$ auf $Y$ kein signifikanter direkter Effekt von X auf Y mehr existiert. Von partieller Mediation spricht man hingegen, wenn zusätzlich zu dem indirekten Effekt noch ein direkter Effekt von $\mathrm{X}$ auf Y besteht. ${ }^{308}$

Die Stärke des Gesamteffektes, der von einer unabhängigen Variable auf eine abhängige Variable wirkt, lässt sich folgendermaßen berechnen: ${ }^{309}$

Gesamteffekt $=$ direkter Effekt + Summe der indirekten Effekte

wobei sich die Stärke eines indirekten Effektes aus dem Produkt der beiden zugehörigen $\beta$ - bzw. $\gamma$-Koeffizienten bemisst. In dem oben dargestellten beispielhaften Modell

Vgl. Bagozzi/Yi (1988), S. 80, Bollen (1989), S. 349, Homburg/Baumgartner (1995a), S. 174 sowie Reinecke (2005), S. 232f.

Vgl. Bagozzi/Yi (1988), S. 81 sowie Homburg/Baumgartner (1995a), S. 172 und 174.

Vgl. Baron/Kenny (1986), S. 1176, Homburg/Klarmann (2006), S. 730 sowie Homburg (2007), S. 47f.

Vgl. auch im Folgenden Fox (1980), Bollen (1989), S. 376ff., Homburg (1992b), S. 544, Homburg/Hildebrandt (1998), S. 27 sowie Reinecke (2005), S. $234 \mathrm{ff}$. 
(vgl. Abbildung 11) beträgt der von $\xi_{1}$ auf $\eta_{2}$ wirkende Gesamteffekt somit $\gamma_{21}+\left(\gamma_{11} \cdot \beta_{21}\right)$.

Für die Signifikanzprüfung mediierender Effekte ist die Kovarianzstrukturanalyse alternativen Analyseverfahren - wie der multiplen Regressionsanalyse, aber auch dem varianzanalytischen PLS-Ansatz für Strukturgleichungsmodelle - überlegen. ${ }^{310}$ Jedoch ist es nicht ausreichend, lediglich die einem indirekten Effekt zugehörigen direkten Effekte einzeln kovarianzstrukturanalytisch auf Signifikanz zu überprüfen, da

"the mediation effect may be significant even if only one direct path is significant, but the second direct path is close to significance. "311

Die Signifikanzprüfung der aus der Mediation resultierenden Effekte erfolgt in der vorliegenden Arbeit daher auf Basis der Methode der so genannten 'bias-corrected bootstrap confidence intervals' und folgt damit dem Ergebnis der Simulationsstudie von Cheung/Lau (2008), die hinsichtlich der Vorgehensweise im Rahmen einer kovarianzstrukturanalytischen Signifikanzprüfung zusammenfassend konstatieren,

"that the bias-corrected bootstrap confidence intervals perform best in testing for mediation [...] effects. “" 312

Das Bootstrapping stellt eine resampling-Technik dar, bei der durch mehrfaches zufälliges Ziehen mit Zurücklegen aus einer bestehenden Stichprobe Unterstichproben generiert werden. Die Aggregation der so erzeugten Unterstichproben ergibt eine Quasi-Grundgesamtheit, bezüglich derer im Anschluss wiederum Signifikanzwerte berechnet werden können. ${ }^{313}$

Vgl. Scholderer/Balderjahn (2006), S. 64, Homburg (2007), S. 48 sowie Iacobucci/Saldanha/ Deng (2007).

311 Cheung/Lau (2008), S. 313-317.

312 Cheung/Lau (2008), S. 296.

313 Vgl. Herrmann/Huber/Kressmann (2006), S. 44 sowie Cheung/Lau (2008), S. 301. Reinecke (2005), S. 16, erläutert diesbezüglich anschaulich: „Bootstraps sind die Schlaufen an CowboyStiefeln, an denen man die Stiefel hochzieht. So wie sich Baron Münchhausen am eigenen Schopf aus dem Sumpf zieht, so versucht man mit Bootstrap-Verfahren in der Statistik eine Prufverteilung für den errechneten Modellfit durch Resimulation der eigenen Daten zu ermitteln." Zum Begriff und zur Methodik des Bootstrapping vgl. daruber hinaus West/Finch/Curran (1995), S. 66f., Byrne (2001), S. 267ff. sowie speziell zur in der vorliegenden Arbeit fur die Berechnung von Konfidenzintervallen angewendeten Perzentilmethode Efron (1987) und Shrout/Bolger (2002), S. $426 \mathrm{ff}$. 
Im Gegensatz zu einem mediierenden Effekt liegt ein moderierender Effekt vor, wenn die Stärke oder sogar die Richtung eines Zusammenhangs zwischen einer unabhängigen und einer abhängigen Variablen von der Ausprägung einer dritten Variablen abhängt. ${ }^{314}$ Letztere stellt die moderierende Variable dar, da sie die Dependenzbeziehung der zwei anderen Variablen beeinflusst - in diesem Zusammenhang wird auch von einem Interaktionseffekt gesprochen. ${ }^{315}$

Die kovarianzstrukturanalytische Überprüfung moderierender Effekte kann zum einen erfolgen, indem ein aus der moderierenden und der unabhängigen Variablen gebildeter multiplikativer Interaktionsterm in das Dependenzmodell integriert wird. ${ }^{316}$ Allerdings führt diese Vorgehensweise im Falle mehrerer Interaktionseffekte zu einer sehr komplexen Struktur der Fehlerterme und ist dann als unpraktikabel einzustufen. ${ }^{317}$ Zum anderen - dieser Ansatz wird in der vorliegenden Arbeit verwendet - kann die Untersuchung moderierender Effekte anhand einer kovarianzstrukturanalytischen Mehrgruppenanalyse vorgenommen werden. ${ }^{318}$ Hierbei wird für zwei aus Teildatensätzen bestehende Gruppen simultan jeweils ein eigenes Modell geschätzt. Die Gruppenbildung erfolgt dabei anhand der Ausprägung derjenigen Variablen, für die ein moderierender Einfluss vermutet wird. Bei diskreten Variablen ist dies besonders einfach - in der vorliegenden empirischen Untersuchung wird bei der Moderationsanalyse beispielsweise nach der Zugehörigkeit zu den Teilnehmergruppen 'Controller' bzw. 'Manager' unterschieden. Bei kontinuierlichen Variablen wird dagegen in der Regel ein Mediansplit der Stichprobe durchgeführt, sodass sich hieraus eine Teilstichprobe mit einer hohen Ausprägung der moderierenden Variablen und eine Teilstichprobe mit einer niedrigen Ausprägung der moderierenden Variablen ergeben.

Vgl. Sharma/Durand/Gur-Arie (1981), S. 292, Arnold (1982), S. 143, Baron/Kenny (1986), S. 1174, Stone (1988), S. 194 sowie Homburg (2007), S. 49.

Vgl. Huber/Heitmann/Herrmann (2006), S. 697.

Vgl. Kenny/Judd (1984), Homburg/Hildebrandt (1998), S. 28, Homburg/Klarmann (2006), S. 730 sowie Scholderer/Balderjahn/Paulssen (2006), S. 644.

Vgl. Homburg/Hildebrandt (1998), S. 28 sowie - auch furr weitere Schwächen dieser Methode Scholderer/Balderjahn/Paulssen (2006), S. 644f.

Vgl. auch im Folgenden Bagozzi/Yi (1988), S. 83, Homburg/Hildebrandt (1998), S. 28f., Pflesser (1999), S. 116-118, Reinecke (2005), S. 238ff., Homburg/Klarmann (2006), S. 730, Huber/Heitmann/Herrmann (2006), S. 697f., Marsh/Wen/Hau (2006), S. 232f., Scholderer/ Balderjahn/Paulssen (2006), S. 643f. sowie Homburg (2007), S. 49f. Ein Überblick über weitere Verfahren zur Analyse von Interaktionseffekten findet sich bei Huber/Heitmann/Herrmann (2006), S. 698ff. sowie Scholderer/Balderjahn/Paulssen (2006), S. 643ff. 
Die Beurteilung der Anpassungsgüte eines solchen simultan geschätzten Mehrgruppenmodells kann anhand der üblichen, bereits vorgestellten Gütekriterien vorgenommen werden. ${ }^{319}$ Ergibt die simultane, unabhängige Schätzung der beiden Modelle deutliche Unterschiede in den jeweiligen standardisierten Effekten des Strukturmodells, so stellt dies ein erstes Anzeichen für das Vorliegen eines moderierenden Effektes dar. Zur Überprüfung wird anschließend ein restringiertes Kovarianzstrukturmodell spezifiziert, d.h. es wird eine Identitätsrestriktion für den interessierenden $\gamma$ bzw. $\beta$-Koeffizienten eingeführt, sodass dieser Koeffizient für beide Gruppen nur noch einmal geschätzt werden muss. Folglich verringert sich die Anzahl der zu schätzenden Modellparameter um eins, wodurch ein zusätzlicher Freiheitsgrad gewonnen wird. Sämtliche übrige Modellparameter werden weiterhin für beide Gruppen unabhängig voneinander geschätzt. Führt nun die restringierte Schätzung des interessierenden Effektes zu einer signifikanten Modellverschlechterung - maßgeblich hierfür ist die Differenz der $\chi^{2}$-Werte -, so gilt das Vorliegen eines moderierenden Effektes als gegeben. ${ }^{320}$

Vgl. Homburg/Hildebrandt (1998), S. 29.

Vgl. Bagozzi/Yi (1988), S. 83, Homburg/Hildebrandt (1998), S. 28f., Pflesser (1999), S. 117f., Homburg/Klarmann (2006), S. 730, Huber/Heitmann/Herrmann (2006), S. 698, Scholderer/ Balderjahn/Paulssen (2006), S. 644 sowie Homburg (2007), S. 49f. Zur Durchfuhrung des $\chi^{2}-$ Differenztests im Rahmen einer kovarianzstrukturanalytischen Mehrgruppenanalyse vgl. Homburg/Dobratz (1998), S. 456f. sowie Reinecke (2005), S. 123. 
Hendrik Angelkort - 978-3-631-75143-5

Downloaded from PubFactory at 01/11/2019 07:47:42AM

via free access 


\section{Status quo der Integration des Rechnungswesens in deutschen Großunternehmen}

Nachdem die Grundlagen der empirischen Untersuchung dargestellt wurden, erfolgt im folgenden Abschnitt die Beantwortung der ersten Forschungsfrage, d.h. die Ermittlung des Status quo der Integration von internem und externem Rechnungswesen. Hierzu werden die zentralen integrationsrelevanten Aspekte innerhalb der verschiedenen Aktionsfelder der Controllerarbeit - diese wurden anhand des House of Controlling in Abschnitt B1.2 vorgestellt - identifiziert und auf Basis der Controllerantworten empirisch erfasst. Im nächsten Schritt wird die Integrationsintensität von internem und externem Rechnungswesen durch Aggregation der Einzelaspekte zu Integrationsindizes ermittelt. Anschließend erfolgt eine Bestandsaufnahme hinsichtlich der Umsetzung des - aus konzeptioneller Sicht vorteilhaft erscheinenden - Musters einer partiellen Integration des Rechnungswesens. ${ }^{321}$

\section{Integration des internen und externen Rechnungswesens innerhalb der Aktionsfelder der Controllerarbeit}

\subsection{Planung}

Im Bereich der Planung ist hinsichtlich der Beurteilung des Status quo der Integration des internen und externen Rechnungswesens zum einen der Planungsinhalt relevant, d.h. die Frage, in welchem Ausmaß innerhalb der kurz- bzw. mittelfristigen Planung die pagatorischen Wertansätze der externen Rechnungslegung verwendet werden. Je umfangreicher dies der Fall ist - und somit je geringer die Bedeutung kalkulatorischer Positionen wie z.B. Standardkosten -, desto höher ist die sich ergebende Integrationsintensität. ${ }^{322}$ Neben dem Planungsinhalt ist zum anderen die Struktur der Planungsobjekte von Interesse, d.h. die Frage, inwieweit die Verantwortungsstruktur in der Ergebnisplanung mit der Legalstruktur des Unternehmens, also der Gesamtheit an rechtlichen Einheiten, übereinstimmt. Die Bereitstellung von finanzberichterstattungskonformen Plandaten der Bestands- und Erfolgsgrößen seitens des Controllings ist im Falle von Überschneidungen und Mehrdeutigkeiten im Verhältnis von Legal- und

321 Zum Muster einer partiellen Integration des Rechnungswesens vgl. die Ausfürungen in Abschnitt B3.2 der vorliegenden Arbeit.

322 Vgl. Haeger (2006), S. 247 sowie Weißenberger/Angelkort (2007a), S. 425. 
Steuerungsstruktur ${ }^{323}$ aufwändig bzw. bei Verwendung kalkulatorischer Kostenarten praktisch nicht möglich und beeinträchtigt somit die Investorenkommunikation. ${ }^{324}$ Ein hohes Ausmaß an Übereinstimmung von Legal- und Steuerungsstruktur stellt daher tendenziell ein Indiz für eine starke Integration des internen und externen Rechungswesens dar. Der Status quo der Integration des Rechnungswesens im Bereich Planung bei den untersuchten Unternehmen der Hauptstichprobe ist in Tabelle 7 dargestellt.

\begin{tabular}{|c|c|c|c|c|c|c|c|c|}
\hline \multirow{3}{*}{$\begin{array}{l}\text { Integration des Rechnungswesens } \\
\text { im Bereich Planung }\end{array}$} & \multirow{3}{*}{ MW } & \multirow{3}{*}{ SA } & \multicolumn{6}{|c|}{ Relative Antworthäufigkeit in \% } \\
\hline & & & 0 & 1 & 2 & 3 & 4 & 5 \\
\hline & & & \multicolumn{2}{|c|}{$\begin{array}{c}\text { (sehr) schwache } \\
\text { Integration }\end{array}$} & \multicolumn{2}{|c|}{$\begin{array}{l}\text { mittelstarke } \\
\text { Integration }\end{array}$} & \multicolumn{2}{|c|}{$\begin{array}{l}\text { (sehr) starke } \\
\text { Integration }\end{array}$} \\
\hline \multirow{2}{*}{$\begin{array}{l}\text { Ausmaß der Verwendung von Wertansätzen der } \\
\text { externen Rechnungslegung in der operativen/kurz- } \\
\text { fristigen Ergebnisplanung auf Gesamtunternehmens-/ } \\
\text { Konzernebene } \\
\qquad(0=\text { sehr gering, ..., } 5=\text { sehr hoch } / N=214)\end{array}$} & \multirow{2}{*}{4,02} & \multirow{2}{*}{1,23} & 2,34 & 4,67 & 3,74 & 11,21 & 34,11 & 43,93 \\
\hline & & & \multicolumn{2}{|c|}{7,01} & \multicolumn{2}{|c|}{14,95} & \multicolumn{2}{|c|}{78,04} \\
\hline \multirow{2}{*}{$\begin{array}{l}\text { Ausmaß der Verwendung von Wertansätzen der } \\
\text { externen Rechnungslegung in der mittelfristigen } \\
\text { Ergebnisplanung auf Gesamtunternehmens-/ } \\
\text { Konzernebene } \\
\qquad(0=\text { sehr gering, ..., } 5=\text { sehr hoch } / N=210)\end{array}$} & \multirow[b]{2}{*}{3,96} & \multirow[b]{2}{*}{1,24} & 2,86 & 3,81 & 4,76 & 12,38 & 35,24 & 40,95 \\
\hline & & & \multicolumn{2}{|c|}{6,67} & \multicolumn{2}{|c|}{17,14} & \multicolumn{2}{|c|}{76,19} \\
\hline \multirow{2}{*}{$\begin{array}{l}\text { Ausmaß der Übereinstimmung der Verantwortungs- } \\
\text { struktur in der operativen Ergebnisplanung mit der } \\
\text { Legalstruktur des Unternehmens } \\
\qquad(0=\text { sehr gering, ..., } 5=\text { sehr hoch } / N=214)\end{array}$} & \multirow{2}{*}{3,27} & \multirow{2}{*}{1,44} & 5,61 & 10,28 & 12,15 & 11,68 & 43,93 & 16,36 \\
\hline & & & \multicolumn{2}{|c|}{15,89} & \multicolumn{2}{|c|}{23,83} & \multicolumn{2}{|c|}{60,29} \\
\hline
\end{tabular}

Tabelle 7: Integration des Rechnungswesens im Bereich Planung

Die Messung erfolgt - wie auch bei den übrigen Aktionsfeldern - für jedes Item auf einer sechsstufigen Rating-Skala, wobei die Antwortabstufung hierbei zunächst fragenspezifisch ausgestaltet ist und jeweils unterhalb der Fragestellung aufgeführt ist. $\mathrm{Da}$ grundsätzlich alle integrationsrelevanten Items derart formuliert sind, dass ein niedriger (hoher) Antwortwert ein Indiz für eine schwache (starke) Integration des Rechnungswesens darstellt, können die erhobenen Daten zudem auf einer ebenfalls sechsstufigen Rating-Skala abgetragen werden, deren Antwortabstufung einheitlich von ' $0=$ sehr schwache Integration' bis ' $5=$ sehr starke Integration' verläuft. Aus Gründen der Übersichtlichkeit wird bei der Darstellung der relativen Antworthäufigkeiten zusätzlich eine Aggregation zu drei verschiedenen Integrationsstufen vorge-

323 In der Unternehmenspraxis wird von so genannten 'Zebra-Gesellschaften' gesprochen, wenn sich Steuerungs- und Legaleinheiten nicht überschneidungsfrei zuordnen lassen, vgl. z.B. Wenzel (2007), S. 300.

324 Vgl. Angelkort/Sandt/Weißenberger (2008b), S. 63 sowie Simons/Weißenberger (2008), S. 140. 
nommen. Diejenigen Unternehmen, deren Antwortwert 0 oder 1 beträgt, zählen im Hinblick auf den jeweils betrachteten Aspekt zur Stufe der sehr schwach bzw. schwach integrierten Unternehmen, während Unternehmen mit einem Antwortwert von 2 bzw. 3 als mittelstark integrierte Unternehmen klassifiziert werden. Die übrigen Unternehmen (Antwortwert 5 oder 6) bilden die Gruppe der stark bzw. sehr stark integrierten Unternehmen.

Sowohl in der operativen als auch in der mittelfristigen Ergebnisplanung weisen die untersuchten Unternehmen mit Mittelwerten von 4,02 bzw. 3,96 insgesamt ein hohes Ausmaß an Integration des internen und externen Rechnungswesens auf; in beiden Fällen verweisen gut drei Viertel der Controller (78,04\% bzw. 76,19\%) auf eine hohe oder sehr hohe Verwendung externer Wertansätze und liefern somit Hinweise auf eine starke bis sehr starke Integration des Rechnungswesens in diesem Bereich. Hinsichtlich der Übereinstimmung der Struktur der Planungsobjekte mit der Legalstruktur zeigt sich mit einem Mittelwert von 3,27 zwar eine etwas schwächere Integration; dennoch weisen auch in diesem Zusammenhang immerhin 60,29\% der antwortenden Unternehmen eine starke oder sehr starke Integration auf.

\subsection{Berichtswesen}

Für das Ausmaß an Integration von internem und externem Rechnungswesen innerhalb des Berichtswesens sind mehrere Aspekte ausschlaggebend. Zunächst ist zu berücksichtigen, inwieweit die interne und externe Ergebnisberichterstattung terminlich miteinander abgestimmt sind, da das Controlling im Rahmen eines integrierten Rechnungswesens für Zwecke der Managementberichterstattung auf Ist-Daten der externen Rechnungslegung zurückgreift und somit abgestimmte Berichtstermine für eine fristgerechte Bereitstellung der benötigten Informationen erforderlich sind. ${ }^{325}$ In engem Zusammenhang hiermit steht als weiteres Indiz für die Ausprägung der Integration die Frage, wie zeitnah das Controlling die auf Basis externer Wertansätze ermittelten Finanzkennzahlen zur Verfügung stellt. Da einerseits viele Unternehmen aus Gründen der Kapitalmarktorientierung ihre externen Reporting-Prozesse - oftmals im Zuge einer IFRS-Einführung - stark beschleunigen ('Fast Close') ${ }^{326}$ und somit auch die für die interne Berichterstattung erforderliche Datenbasis im Rahmen einer integrierten 
Rechnungslegung zeitnah bereitstellen ${ }^{327}$ und andererseits die aus einer Integration resultierende geringere Abstimmungsnotwendigkeit die interne Berichterstattung beschleunigt, ${ }^{328}$ deutet eine zeitnahe Bereitstellung der monatsbezogenen Key Performance Indicators (KPIs) auf eine starke Integration des Rechnungswesens hin.

Das Ausmaß der Verwendung von kalkulatorischen Kosten bzw. Erlösen im Rahmen der laufenden Unternehmenssteuerung bildet - ähnlich wie innerhalb der Planung ${ }^{329}$ - einen weiteren Anhaltspunkt hinsichtlich der Intensität der Integration des Rechnungswesens im Berichtswesen. Diese ist umso höher, je weniger kalkulatorische Positionen verrechnet werden. ${ }^{330}$ Des Weiteren ist die Frage relevant, wie stark die interne Ergebnisrechnung mit der externen publizierten Ergebnisrechnung (GuV) in Einklang steht. Indizien hierfür liegen in der Überführbarkeit von einzelnen Positionen zwischen beiden Ergebnisrechnungen, ${ }^{331}$ dem Ausmaß der Übereinstimmung zwischen dem intern ermittelten und dem extern publizierten operativen Ergebnis sowie zwischen dem zentralen Key Performance Indicator und dem extern publizierten operativen Ergebnis. Die besondere Bedeutung des operativen Ergebnisses ergibt sich hierbei aus der hohen Relevanz dieser Kennzahl für die laufende Steuerung, entweder als Eingangsgröße bei der Ermittlung des KPI oder in direkter Verwendung als KPI. ${ }^{332}$ Bei dieser zentralen zur Unternehmenssteuerung verwendeten internen Ergebnisgröße ist hinsichtlich der Abweichung vom operativen Ergebnis gemäß GuV sowohl die Anzahl der Überleitungspositionen als auch das wertmäßige Volumen, welches diese Anpassungen in Summe ausmachen, zu berücksichtigen, da beide Aspekte eine Vergleichbarkeit der intern ermittelten Steuerungsgröße mit dem GuV-Wert erschweren und folglich ceteris paribus mit einer schwächeren Integrationsintensität einhergehen.

Tabelle 8 zeigt den Status quo der Integration des Rechnungswesens im Bereich Berichtswesen bei den untersuchten Unternehmen der Hauptstichprobe.

Vgl. Weißenberger (2007a), S. 217 sowie Weißenberger/Angelkort (2007a), S. 415.

Vgl. Erichsen (2000), S. 58 sowie Wagenhofer (2006), S. 12f.

Vgl. hierzu die Ausführung in Abschnitt D1.1 der vorliegenden Arbeit.

Diese Frage stellt somit - genau wie das zweite sowie die beiden letzten Items innerhalb des Berichtswesens - ein so genanntes 'reverse coded item' dar, da eine hohe Ausprägung hinsichtlich des erfragten Sachverhalts (hier: Verwendung kalkulatorischer Positionen) einem niedrigen zugeordneten Skalenwert entspricht (vgl. Tabelle 8).

Vgl. hierzu Dais/Watterott (2006), S. 470f., Weißenberger (2008a), Rz. 58 sowie - mit besonderem Fokus auf die Abstimmbarkeit von Angaben der Segmentberichterstattung und der internen Ergebnisrechnung - Franz/Winkler (2006), S. 87-91.

Vgl. Kley (2006), S. 154. 


\begin{tabular}{|c|c|c|c|c|c|c|c|c|}
\hline \multirow{3}{*}{$\begin{array}{l}\text { Integration des Rechnungswesens } \\
\text { im Bereich Berichtswesen }\end{array}$} & \multirow{3}{*}{ MW } & \multirow{3}{*}{ SA } & \multicolumn{6}{|c|}{ Relative Antworthäufigkeit in \% } \\
\hline & & & 0 & 1 & 2 & 3 & 4 & 5 \\
\hline & & & \multicolumn{2}{|c|}{$\begin{array}{c}\text { (sehr) schwache } \\
\text { Integration }\end{array}$} & \multicolumn{2}{|c|}{$\begin{array}{l}\text { mittelstarke } \\
\text { Integration }\end{array}$} & \multicolumn{2}{|c|}{$\begin{array}{l}\text { (sehr) starke } \\
\text { Integration }\end{array}$} \\
\hline \multirow{2}{*}{$\begin{array}{l}\text { Inwieweit sind die Termine der internen und externen } \\
\text { Ergebnisberichterstattung miteinander abgestimmt? } \\
\qquad(0=\text { sehr wenig, } \ldots, 5=\text { sehr stark } / N=214)\end{array}$} & \multirow{2}{*}{4,26} & \multirow{2}{*}{1,04} & 0,93 & 1,87 & 5,14 & 7,94 & 30,37 & 53,74 \\
\hline & & & \multicolumn{2}{|c|}{2,80} & \multicolumn{2}{|c|}{13,08} & \multicolumn{2}{|c|}{84,11} \\
\hline \multirow{2}{*}{$\begin{array}{l}\text { Wie viele Arbeitstage (AT) nach Ultimo legt das Controlling } \\
\text { dem Top-Management die zentralen monatsbezogenen } \\
\text { Finanzkennzahlen (KPIs) - ermittelt auf Basis der } \\
\text { Wertansätze der externen Rechnungslegung - vor? } \\
\qquad \begin{aligned}(0= & \text { KPIs werden nicht auf Basis der } \\
& \text { Wertansätze der externen } \\
& \text { Rechnungslegung ermittelt } \\
1 & =>20 A T \\
2 & =13-20 A T \\
3 & =7-12 A T \\
4 & =4-6 A T \\
5 & =1-3 A T / N=213)\end{aligned}\end{array}$} & \multirow{2}{*}{2,85} & \multirow{2}{*}{1,37} & 12,68 & 4,23 & 7,51 & 42,72 & 27,23 & 5,63 \\
\hline & & & \multicolumn{2}{|c|}{16,91} & \multicolumn{2}{|c|}{50,23} & \multicolumn{2}{|c|}{32,86} \\
\hline \multirow{2}{*}{$\begin{array}{l}\text { Wie hoch ist die Bedeutung kalkulatorischer Kosten/Erlöse } \\
\text { für die laufende Steuerung auf Gesamtunternehmens-/ } \\
\text { Konzernebene? } \\
\qquad(0=\text { sehr hoch, ..., } 5=\text { sehr gering } / N=213)\end{array}$} & \multirow{2}{*}{3,62} & \multirow{2}{*}{1,48} & 3,76 & 9,86 & 9,39 & 10,80 & 30,05 & 36,15 \\
\hline & & & \multicolumn{2}{|c|}{13,62} & \multicolumn{2}{|c|}{20,19} & \multicolumn{2}{|c|}{66,20} \\
\hline \multirow{2}{*}{$\begin{array}{l}\text { In welchem Ausmaß lassen sich einzelne Zeilen/ } \\
\text { Zwischensummen des internen Ergebnisberichts in } \\
\text { Zeilen/Zwischenergebnisse der externen Ergebnis- } \\
\text { rechnung (= GuV) überführen? } \\
\qquad(0=\text { sehr wenig, ..., } 5=\text { sehr stark / } N=214)\end{array}$} & \multirow{2}{*}{3,79} & \multirow{2}{*}{1,34} & 2,34 & 6,54 & 8,88 & 12,62 & 31,31 & 38,32 \\
\hline & & & \multicolumn{2}{|c|}{8,88} & \multicolumn{2}{|c|}{21,50} & \multicolumn{2}{|c|}{69,63} \\
\hline \multirow{2}{*}{$\begin{array}{l}\text { Wie hoch ist die betragsmäßige Übereinstimmung } \\
\text { zwischen dem intern ermittelten operativen Ergebnis und } \\
\text { dem in der externen Finanzberichterstattung publizierten } \\
\text { operativen Ergebnis (d.h. vor Zinsen und Steuern) auf } \\
\text { Gesamtunternehmens-/Konzernebene? } \\
\qquad(0=\text { sehr gering, ..., } 5=\text { sehr hoch } / N=213)\end{array}$} & \multirow{2}{*}{4,19} & \multirow{2}{*}{1,05} & 0,00 & 3,76 & 4,69 & 10,80 & 30,05 & 50,70 \\
\hline & & & \multicolumn{2}{|c|}{3,76} & \multicolumn{2}{|c|}{15,49} & 80 & 75 \\
\hline $\begin{array}{l}\text { Wie stark weicht die zentrale zur Unternehmens- } \\
\text { steuerung verwendete interne Ergebnisgröße (z.B. EBIT, } \\
\text { EBITDA, etc.) von dem in der externen Finanzbericht- } \\
\text { erstattung publizierten operativen Ergebnis ab hinsichtlich } \\
\text { der Anzahl an typischerweise vorgenommenen } \\
\text { Bereinigungen/Anpassungen? }\end{array}$ & & & 7,25 & 3,86 & 11,59 & 20,29 & 32,85 & 24,15 \\
\hline $\begin{aligned}(0 & =>10 \text { Bereinigungen/Anpassungen } \\
1 & =8-10 \text { Bereinigungen/Anpassungen } \\
2 & =5-7 \text { Bereinigungen/Anpassungen } \\
3 & =3-4 \text { Bereinigungen/Anpassungen } \\
4 & =1-2 \text { Bereinigungen/Anpassungen } \\
5 & =0 \text { Bereinigungen/Anpassungen } / N=207)\end{aligned}$ & 3,40 & 1,44 & & & & & & ,00 \\
\hline $\begin{array}{l}\text { Wie stark weicht die zentrale zur Unternehmens- } \\
\text { steuerung verwendete interne Ergebnisgröße (z.B. EBIT, } \\
\text { EBITDA, etc.) von dem in der externen Finanzbericht- } \\
\text { erstattung publizierten operativen Ergebnis ab hinsichtlich }\end{array}$ & 38 & 1 & 0,96 & 5,26 & 7,18 & 18,18 & 31,10 & 37,32 \\
\hline $\begin{array}{l}\text { des wertmäßigen Volumens der typischerweise } \\
\text { vorgenommenen Bereinigungen/Anpassungen? } \\
\qquad(0=\text { sehr stark, ..., } 5=\text { sehr wenig } / N=209)\end{array}$ & (1) & & & & & & & 42 \\
\hline
\end{tabular}

Tabelle 8: Integration des Rechnungswesens im Bereich Berichtswesen 
Auch das Berichtswesen der betrachteten Unternehmen ist insgesamt durch ein hohes Ausmaß an Integration des internen und externen Rechnungswesens gekennzeichnet. Insbesondere bei der terminlichen Abstimmung der internen und externen Ergebnisberichterstattung sowie bezüglich der betragsmäßigen Übereinstimmung des intern ermittelten und des extern ausgewiesenen operativen Ergebnisses deutet der überwiegende Anteil der Controllerantworten (84,11\% bzw. 80,75\%) auf eine starke bis sehr starke Integration des Rechnungswesens hin; der jeweilige Mittelwert liegt hier bei 4,26 bzw. 4,19. Hinsichtlich des Aspekts einer zeitnahen Berichterstattung von auf Basis externer Wertansätze ermittelten Finanzkennzahlen hingegen weisen die antwortenden Unternehmen mehrheitlich $(50,23 \%)$ lediglich eine mittelstarke Integration auf, was sich auch im deutlich niedrigeren Mittelwert in Höhe von 2,85 widerspiegelt. ${ }^{333}$

\subsection{Performance-Messung}

Im Aktionsfeld Performance-Messung steht die Frage im Mittelpunkt der Betrachtung, wie stark die Gesamtvergütung der Unternehmensleitung von Ergebnisgrößen der externen Finanzberichterstattung abhängt. Hiermit wird der seitens der Anteilseigner zunehmend aufgestellten Forderung Rechnung getragen, die Leistung des Managements anhand derselben Daten zu beurteilen, die den externen Bilanzadressaten zur Verfügung gestellt werden. ${ }^{334}$ Auf diese Art und Weise können Erfolgserwartungen der Investoren in interne Zielvorgaben umgesetzt und mit dem Vergütungs- bzw. Performance-Messungssystem verknüpft werden. ${ }^{335}$ Ein hoher Anteil einer auf externen und (somit pagatorischen) Ergebnisgrößen basierenden variablen Vergütung an der Gesamtvergütung stellt in diesem Zusammenhang ein Indiz für eine starke Integration des Rechnungswesens dar.

Wie Tabelle 9 zeigt, weisen - bei einem Mittelwert in Höhe von 3,65 - etwa zwei Drittel $(66,04 \%)$ der an der Studie beteiligten Unternehmen eine starke bis sehr starke Integration des Rechnungswesens innerhalb der Performance-Messung auf.

333 Hierbei ist jedoch zu beachten, dass sich die Antwortabstufungen des zweiten sowie des vorletzten Items - bei beiden Fragen hat sich ein eher niedriger Mittelwert ergeben - in ihrer Art jeweils von der Codierung der übrigen Items unterscheiden. Die Aussagekraft eines direkten Vergleiches der Mittelwerte ist daher an dieser Stelle eingeschränkt.

335 Vgl. Heyd (2001), S. 203 sowie Weißenberger (2008a), Rz. 61. 


\begin{tabular}{|c|c|c|c|c|c|c|c|c|}
\hline \multirow{3}{*}{$\begin{array}{l}\text { Integration des Rechnungswesens } \\
\text { im Bereich Performance-Messung }\end{array}$} & \multirow{3}{*}{ MW } & \multirow{3}{*}{ SA } & \multicolumn{6}{|c|}{ Relative Antworthäufigkeit in \% } \\
\hline & & & 0 & 1 & 2 & 3 & 4 & 5 \\
\hline & & & \multicolumn{2}{|c|}{\begin{tabular}{|c|}
$\begin{array}{c}\text { (sehr) schwache } \\
\text { Integration }\end{array}$ \\
\end{tabular}} & \multicolumn{2}{|c|}{$\begin{array}{l}\text { mittelstarke } \\
\text { Integration }\end{array}$} & \multicolumn{2}{|c|}{$\begin{array}{l}\text { (sehr) starke } \\
\text { Integration }\end{array}$} \\
\hline \multirow{2}{*}{$\begin{array}{l}\text { Wie stark hängt die Gesamtvergütung des Top- } \\
\text { Managements von Ergebnisgrößen der externen } \\
\text { Finanzberichterstattung ab? } \\
\qquad(0=\text { sehr schwach, ..., } 5=\text { sehr stark } / N=212)\end{array}$} & \multirow{2}{*}{3,65} & \multirow{2}{*}{1,35} & 4,25 & 5,66 & 7,08 & 16,98 & 36,32 & 29,72 \\
\hline & & & \multicolumn{2}{|c|}{9,91} & \multicolumn{2}{|c|}{24,06} & \multicolumn{2}{|c|}{66,04} \\
\hline
\end{tabular}

Tabelle 9: Integration des Rechnungswesens im Bereich Performance-Messung

\subsection{Gestaltung der controllingrelevanten IT-Systeme}

Für die Beurteilung des Status quo der Integration von internem und externem Rechnungswesen im Bereich Gestaltung der controllingrelevanten IT-Systeme ist die systemtechnische Ausgestaltung der Datenerfassung, -haltung und -analyse bei den teilnehmenden Unternehmen genauer zu betrachten. Zunächst ist die Frage relevant, ob im Rahmen der Datenerfassung durch die Finanzbuchhaltung ein gemeinsamer Kontenplan für das interne und externe Rechnungswesen - etwa durch Hinterlegung innerhalb eines Enterprise Resource Planning (ERP)-Systems wie SAP - verwendet wird (Einkreissystem) ${ }^{336}$ Hinsichtlich der Datenhaltung gilt es zu untersuchen, ob die Unternehmen Datenbanken vorhalten, die sowohl dem internen als auch dem externen Rechnungswesen den Zugriff auf die Ist- bzw. Plandaten aus den transaktionalen Vorsystemen aus Finanzbuchhaltung und Planung ermöglichen - etwa basierend auf Data Warehouse-Technologien wie z.B. dem Business Warehouse von SAP. ${ }^{337}$ Als integrationsrelevanter Aspekt in Bezug auf die Systemlandschaft der Datenanalyse ist zudem zu hinterfragen, ob ein integriertes IT-System - wie z.B. SAP SEM - vorliegt, welches neben der internen Managementerfolgsrechnung auch die Konsolidierung der externen Finanzberichterstattung abdeckt. ${ }^{338}$

Die genannten Aspekte bilden die systemtechnische Voraussetzung für eine einheitliche Datenbasis für Zwecke des internen und externen Rechnungswesens und

Vgl. Schmitz (2006), S. 12 sowie Weißenberger (2007a), S. 217.

Vgl. Fleischer (2005), S. 194.

Vgl. Haeger (2006), S. 261f. sowie Krey (2007), S. 231. Für eine ausfuhrliche Darstellung der Softwarelðsungen des SAP-Konzerns im Rahmen eines integrierten Rechnungswesens vgl. Karl (2006). 
geben folglich Aufschluss hinsichtlich des diesbezüglichen Integrationsstands des Rechnungswesens. ${ }^{339}$

Der für den Bereich Gestaltung der controllingrelevanten IT-Systeme ermittelte Status quo der Integration des Rechnungswesens bei den untersuchten Unternehmen der Hauptstichprobe ist in Tabelle 10 dargestellt.

\begin{tabular}{|c|c|c|c|c|c|c|c|c|}
\hline \multirow{3}{*}{$\begin{array}{l}\text { Integration des Rechnungswesens } \\
\text { im Bereich Gestaltung der } \\
\text { controllingrelevanten IT-Systeme }\end{array}$} & \multirow{3}{*}{ MW } & \multirow{3}{*}{ SA } & \multicolumn{6}{|c|}{ Relative Antworthäufigkeit in \% } \\
\hline & & & 0 & 1 & 2 & 3 & 4 & 5 \\
\hline & & & \multicolumn{2}{|c|}{$\begin{array}{c}\text { (sehr) schwache } \\
\text { Integration }\end{array}$} & \multicolumn{2}{|c|}{$\begin{array}{l}\text { mittelstarke } \\
\text { Integration }\end{array}$} & \multicolumn{2}{|c|}{$\begin{array}{l}\text { (sehr) starke } \\
\text { Integration }\end{array}$} \\
\hline \multirow{2}{*}{$\begin{array}{l}\text { In unserem Unternehmen wird unternehmensweit ein } \\
\text { gemeinsamer Kontenplan für das interne und das } \\
\text { externe Rechnungswesen verwendet. } \\
\quad(0=\text { trifft gar nicht } z u, \ldots, 5=\text { trifft voll } z u / N=214)\end{array}$} & \multirow{2}{*}{4,15} & \multirow{2}{*}{1,26} & 1,87 & 4,67 & 5,61 & 8,88 & 22,43 & 56,54 \\
\hline & & & \multicolumn{2}{|c|}{6,54} & \multicolumn{2}{|c|}{14,49} & \multicolumn{2}{|c|}{78,97} \\
\hline \multirow{2}{*}{$\begin{array}{l}\text { In unserem Unternehmen existiert eine oder mehrere } \\
\text { Datenbanken für Ist- und Plan-Daten, auf die sowohl für } \\
\text { Zwecke des internen als auch externen } \\
\text { Rechnungswesens zurückgegriffen werden kann. } \\
\quad(0=\text { trifft gar nicht } z u, \ldots, 5=\text { trifft voll } z u / N=214)\end{array}$} & \multirow[b]{2}{*}{3,86} & \multirow[b]{2}{*}{1,52} & 7,48 & 3,74 & 5,61 & 9,35 & 26,17 & 47,66 \\
\hline & & & \multicolumn{2}{|c|}{11,22} & \multicolumn{2}{|c|}{14,96} & \multicolumn{2}{|c|}{73,83} \\
\hline \multirow{2}{*}{$\begin{array}{l}\text { In unserem Unternehmen existiert ein integriertes IT- } \\
\text { System (z.B. SAP-SEM), das sowohl die interne } \\
\text { Managementerfolgsrechnung als auch die Konsolidierung } \\
\text { der externen Finanzberichterstattung abbildet. } \\
\qquad(0=\text { trifft gar nicht } z u, \ldots, 5=\text { trifft voll } z u / N=214)\end{array}$} & \multirow[b]{2}{*}{2,78} & \multirow[b]{2}{*}{1,85} & 18,22 & 13,08 & 10,28 & 14,95 & 18,22 & 25,23 \\
\hline & & & \multicolumn{2}{|c|}{31,30} & \multicolumn{2}{|c|}{25,23} & \multicolumn{2}{|c|}{43,45} \\
\hline
\end{tabular}

Tabelle 10: Integration des Rechnungswesens im Bereich Gestaltung der controllingrelevanten IT-Systeme

Während die Controllerantworten hinsichtlich der Datenerfassung bzw. der Datenhaltung überwiegend (78,97\% bzw. 73,83\%) auf eine starke bis sehr starke Integration des Rechnungswesens hinweisen und mit 4,15 bzw. 3,86 auch hohe Mittelwerte aufweisen, ergibt sich bezüglich der Datenanalyse ein differenzierteres Bild. Bei knapp einem Drittel $(31,30 \%)$ der Unternehmen zeigt sich hier eine schwache bis sehr schwache Integration; zugleich werden von $43,45 \%$ der Unternehmen Angaben gemacht, die auf eine starke bis sehr starke Integration schließen lassen. Die hohe Bandbreite der Antworten spiegelt sich folglich in einer vergleichsweise hohen Standardabweichung von 1,85 wider. Insgesamt ergibt sich für den Aspekt der Datenanalyse mit einem Mittelwert von 2,78 lediglich eine mittelstarke Integration von internem und externem Rechnungswesen bei den untersuchten Unternehmen. 


\subsection{Institutionalisierung der Controllerarbeit}

Im Aktionsfeld der Institutionalisierung der Controllerarbeit sind sowohl aufbau- als auch ablauforganisatorische Aspekte für die Bestandsaufnahme der Integration von internem und externem Rechnungswesen von Bedeutung. Hinsichtlich der Aufbauorganisation gilt es zu berücksichtigen, ob bei den teilnehmenden Unternehmen Controlling und Bilanzierung dem gleichen Vorstands- bzw. Geschäftsführungsressort unterstellt sind. So werden im Falle einer Bündelung dieser beiden Finanzfunktionen in einem Vorstandsressort - etwa das des Chief Financial Officer (CFO) - Konfliktpotenziale gesenkt und somit der Weg für eine effiziente Ablauforganisation geebnet. ${ }^{340}$

Diese zeichnet sich bei stark integrierten Unternehmen durch einen direkten Informations- und Erfahrungsaustausch hinsichtlich Fach- und Methodenthemen auf der Grundlage institutionalisierter Kommunikationswege, etwa in Form regelmäßig stattfindender gemeinsamer Schulungsveranstaltungen bzw. Sitzungen, aus. ${ }^{341}$ Ein weiteres Indiz für die Integrationsintensität des Rechnungswesens in diesem Bereich liegt zudem in der Frage, inwiefern das Controlling auch die Bilanzierer über Berichtsinhalte informiert. Diese müssen sich in zunehmendem Maße Hintergrundwissen hinsichtlich der operativen Geschäftsentwicklung aneignen, um die Abschlusszahlen aus betriebswirtschaftlicher Sicht interpretieren zu können. ${ }^{342}$

Tabelle 11 zeigt den Status quo der Integration des Rechnungswesens im Bereich Institutionalisierung der Controllerarbeit bei den untersuchten Unternehmen der Hauptstichprobe. Insgesamt weisen die Controllerantworten auf ein hohes Ausmaß an Integration in diesem Aktionsfeld hin, wenngleich auch eine deutliche Diskrepanz zwischen dem aufbauorganisatorischen Aspekt einerseits und den beiden ablauforganisatorischen Kriterien andererseits vorliegt. Während die Unternehmen aus aufbauorganisatorischer Sicht nahezu ausschließlich (92,52\%) eine starke bzw. sehr starke Integration des Rechnungswesens aufweisen, zeigt sich hinsichtlich der Ablauforganisation bei jeweils ca. einem Viertel der Fälle (24,77\% bzw. 28,51\%) lediglich eine mittelstarke Integration. Folglich ergeben sich hier auch deutlich niedrigere Mittelwerte als im ersten Fall (3,95 bzw. 3,77 im Vergleich zu 4,67). 


\begin{tabular}{|c|c|c|c|c|c|c|c|c|}
\hline \multirow{3}{*}{$\begin{array}{l}\text { Integration des Rechnungswesens } \\
\text { im Bereich Institutionalisierung der } \\
\text { Controllerarbelt }\end{array}$} & \multirow{3}{*}{ MW } & \multirow{3}{*}{ SA } & \multicolumn{6}{|c|}{ Relative Antworthăufigkeit in \% } \\
\hline & & & 0 & 1 & 2 & 3 & 4 & 5 \\
\hline & & & \multicolumn{2}{|c|}{$\begin{array}{l}\text { (sehr) schwache } \\
\text { Integration }\end{array}$} & \multicolumn{2}{|c|}{$\begin{array}{l}\text { mittelstarke } \\
\text { Integration }\end{array}$} & \multicolumn{2}{|c|}{$\begin{array}{l}\text { (sehr) starke } \\
\text { Integration }\end{array}$} \\
\hline \multirow{2}{*}{$\begin{array}{l}\text { Controlling und Bilanzierung sind in unserem Unterneh- } \\
\text { men aufbauorganisatorisch dem gleichen Vorstands- } \\
\text { bzw. Geschäftsführungsressort unterstellt. } \\
\qquad(0=\text { trifft gar nicht } z u, \ldots, 5=\text { trifft voll } z u / N=214)\end{array}$} & \multirow{2}{*}{4,67} & \multirow{2}{*}{1,02} & 2,80 & 0,93 & 1,40 & 2,34 & 6,54 & 85,98 \\
\hline & & & \multicolumn{2}{|c|}{3,73} & \multicolumn{2}{|c|}{3,74} & \multicolumn{2}{|c|}{92,52} \\
\hline \multirow{2}{*}{$\begin{array}{l}\text { Zwischen Controllern und Bilanzierern gibt es in unserem } \\
\text { Unternehmen einen intensiven Austausch über Fach- und } \\
\text { Methodenthemen. } \\
\quad(O=\text { trifft gor nicht } z u, \ldots, 5=\text { trifft voll } z u / N=214)\end{array}$} & \multirow{2}{*}{3,95} & \multirow{2}{*}{1,06} & 0,00 & 3,27 & 7,01 & 17,76 & 35,51 & 36,45 \\
\hline & & & \multicolumn{2}{|c|}{3,27} & \multicolumn{2}{|c|}{24,77} & \multicolumn{2}{|c|}{71,96} \\
\hline \multirow{2}{*}{$\begin{array}{l}\text { In unserem Unternehmen informiert das Controlling auch } \\
\text { die Bilanzierung ausführlich über Inhalte der Manage- } \\
\text { mentberichterstattung. } \\
\quad(0=\text { trifft gar nicht } z u, \ldots, 5=\text { trifft voll } z u / N=214)\end{array}$} & \multirow{2}{*}{3,77} & \multirow{2}{*}{1,17} & 0,00 & 6,07 & 8,88 & 19,63 & 33,18 & 32,24 \\
\hline & & & \multicolumn{2}{|c|}{6,07} & \multicolumn{2}{|c|}{28,51} & \multicolumn{2}{|c|}{65,42} \\
\hline
\end{tabular}

Tabelle 11: Integration des Rechnungswesens im Bereich Institutionalisierung der Controllerarbeit

\section{Bildung von Integrationsindizes als Maß der Integrations- intensität von internem und externem Rechnungswesen}

\subsection{Vorgehensweise bei der Indexbildung}

Nachdem in Abschnitt D1 die verschiedenen integrationsrelevanten Aspekte für jedes Aktionsfeld der Controllerarbeit detailliert erörtert wurden, sollen hierauf aufbauend im nächsten Schritt sechs verschiedene Integrationsindizes gebildet werden, in denen die Einzelaspekte in unterschiedlichen Aggregationsstufen verdichtet werden. ${ }^{343}$ Die sich hieraus ergebenden Werte fungieren als $\mathrm{Ma} ß$ der Integrationsintensität des internen und externen Rechnungswesens und sind für die vorliegende Arbeit von besonderer Bedeutung, da sie zum einen den Status quo der Integration von internem und externem Rechnungswesen in aggregierter Form abbilden und somit in direkter Weise der Beantwortung der ersten Forschungsfrage dienen. Zum anderen werden dieselben Indizes in den weiteren empirischen Analysen im Rahmen der Beantwortung der

343 Ein Index ist nach Bortz/Döring (2006), S. 143, definiert als „Messwert für ein komplexes Merkmal, der aus den Messwerten mehrerer Indikatorvariablen zusammengesetzt wird.“ $\mathrm{Zu}$ unterschiedlichen Vorgehensweisen im Rahmen der Indexbildung vgl. Bortz/Döring (2006), S. 143ff., Schnell/Hill/Esser (2008), S. 166ff. sowie Mayer (2009), S. 85f. 
zweiten und dritten Forschungsfrage wiederholt als Eingangsgrößen bzw. unabhängige Variablen verwendet.

Zunächst wird für jedes der fünf Aktionsfelder ein separater Integrationssubindex gebildet, indem die Antwortwerte der jeweils zugehörigen einzelnen Items addiert und anschließend durch die Anzahl der Fragen dividiert werden. Für die sich hieraus ergebenden Integrationsindizes sind - ebenso wie bei den jeweils in sie einfließenden Items - definitionsgemäß Werte zwischen „0= sehr schwache Integration“ und , 5 = sehr starke Integration" möglich, wobei sich aufgrund der Mittelwertbildung nun auch nicht ganzzahlige Werte ergeben können. ${ }^{344}$ In den wenigen Fällen, in denen bei Fragen einzelne Antworten seitens der teilnehmenden Controller fehlen, erfolgt die Berechnung des jeweiligen Indexes auf Basis des reduzierten Sets an Einzelitems. ${ }^{345}$

Neben der Berechnung für jedes einzelne Aktionsfeld wird zudem ein Gesamtintegrationsindex gebildet, der für jedes Unternehmen in komprimierter Form darüber Auskunft gibt, in welchem Ausmaß internes und externes Rechnungswesen insgesamt und über alle Aktionsfelder bzw. integrationsrelevanten Fragestellungen hinweg integriert sind. Hierzu wird der Mittelwert der Antwortwerte aller 17 Items berechnet. Da je Aktionsfeld eine unterschiedliche Anzahl integrationsrelevanter Fragestellungen identifiziert wurde, ergibt sich folglich eine unterschiedlich starke Berücksichtigung der fünf Aktionsfelder innerhalb des Gesamtintegrationsindexes. So machen bspw. die Aspekte innerhalb des Berichtswesens einen höheren Anteil (7 von 17) an der Gesamtintegrationsintensität aus als Fragestellungen aus den Aktionsfeldern PerformanceMessung (1 von 17) oder Institutionalisierung der Controllerarbeit (3 von 17). Diese unterschiedliche Gewichtung spiegelt somit die unterschiedlich starke Bedeutung der

344 Die einzige Ausnahme bildet diesbezüglich das Aktionsfeld Performance-Messung, da hier nur ein einzelnes integrationsrelevantes Item identifiziert wurde, vgl. Tabelle 9. Der Integrationsindex entspricht hier folglich dem ganzzahligen Antwortwert dieses Items.

Zwar liegt in dieser Vorgehensweise eine geringfugige Ungenauigkeit, jedoch erscheint sie vor dem Hintergrund vertretbar, dass eine komplette Nichtberücksichtigung von Unternehmen lediglich aufgrund des Fehlens einer einzigen bzw. nur weniger Angaben die Fallzahl der Haupt- und dyadischen Stichprobe reduziert und die Aussagekraft der empirischen Ergebnisse aufgrund des einhergehenden Informationsverlusts folglich beeinträchtigt hätte, vgl. Reinecke (2005), S. 287. Zum Umgang mit fehlenden Werten im Rahmen der empirischen Sozialforschung vgl. grundsătzlich Frane (1976), Schnell/Hill/Esser (2008), S. 468ff. sowie mit besonderem Fokus auf Strukturgleichungsmodelle Byrne (2001), S. 287ff., Reinecke (2005), S. 283ff. und Backhaus/ Blechschmidt (2009). 
verschiedenen Aktionsfelder hinsichtlich des Status quo der Integration von internem und externem Rechnungswesen wider.

\subsection{Integrationsintensität der Aktionsfelder der Controllerarbeit}

Die Ergebnisse der nach Maßgabe der soeben beschriebenen Vorgehensweise durchgeführten Ermittlung der Integrationsindizes zeigen abermals - nun in aggregierter Form -, dass die untersuchten Unternehmen der Hauptstichprobe ein insgesamt hohes $\mathrm{Ma}$ an Integration des internen und externen Rechnungswesens aufweisen (vgl. Abbildung 12).

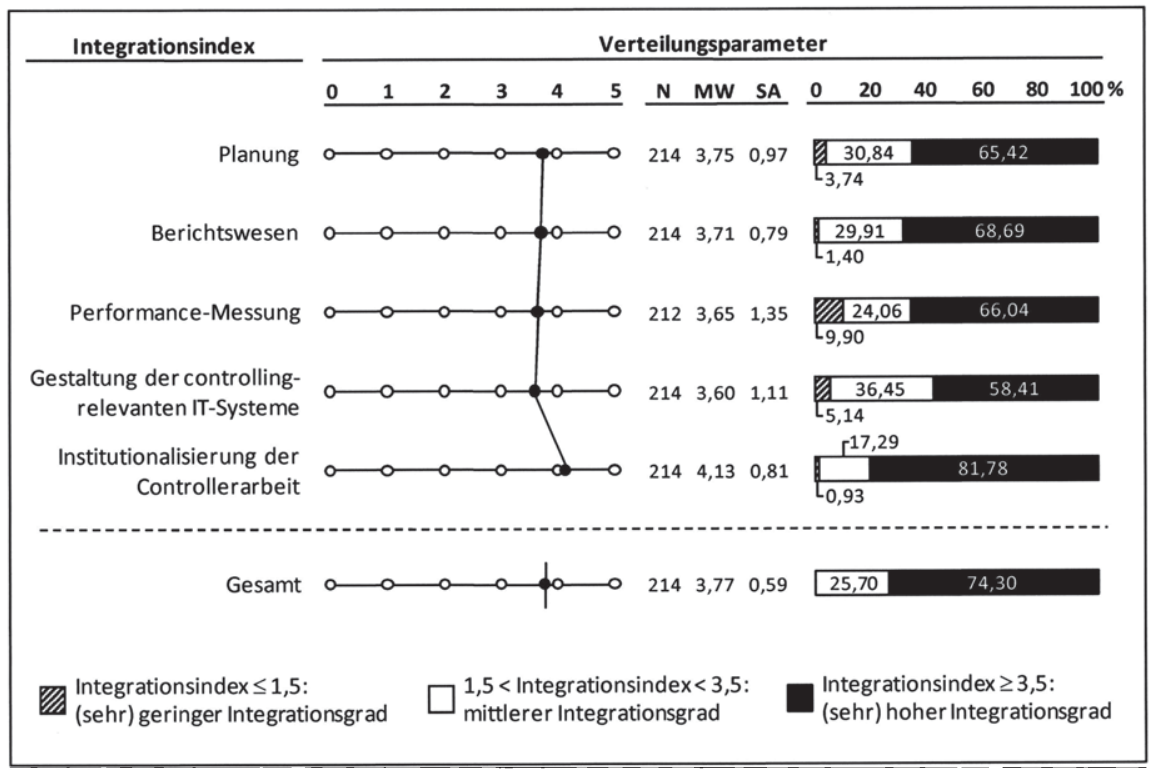

Abbildung 12: Gesamtintegrationsindex und Integrationssubindizes der Aktionsfelder der Controllerarbeit

So weisen ca. drei Viertel $(74,30 \%)$ der Unternehmen einen Gesamtintegrationsindex von mindestens 3,5 und somit einen hohen bis sehr hohen Integrationsgrad auf, während die übrigen Unternehmen $(25,70 \%)$ immerhin durch einen mittleren Integrationsgrad charakterisiert sind. Der durchschnittliche Wert des Gesamtintegrationsindexes liegt mit 3,77 im Bereich hoher bis sehr hoher Integration und unterstreicht somit die eingangs genannte Einschätzung bezüglich des Status quo der Integration des Rechnungswesens. 
Bezogen auf die einzelnen Aktionsfelder zeigt sich ein recht ausgeglichenes Integrationsniveau innerhalb der originären Aktionsfelder Planung, Berichtswesen und Performance-Messung. Die Mittelwerte der jeweiligen Subindizes bewegen sich hier von 3,75 über 3,71 bis 3,65. Jeweils ca. zwei Drittel der Unternehmen $(65,42 \%$ bzw. $68,69 \%$ bzw. $66,04 \%$ ) zeichnen sich durch einen hohen oder sehr hohen Integrationsgrad aus.

Ein differenzierteres Bild ergibt sich bei den beiden derivativen Aktionsfeldern. Im Bereich Gestaltung der controllingrelevanten IT-Systeme erreicht - bei einem Mittelwert von 3,60 - ein vergleichsweise hoher Anteil von 36,45\% der Unternehmen einen nur mittelstarken Integrationsgrad; lediglich 58,41\% der Unternehmen zeichnet sich hier indes durch eine hohe oder sehr hohe Integrationsintensität des Rechnungswesens aus. Innerhalb der Institutionalisierung der Controllerarbeit ist dies dagegen bei $\mathbf{8 1 , 7 8 \%}$ der Unternehmen der Fall; der sich hier ergebende Mittelwert des Integrationssubindexes fällt mit 4,13 entsprechend sehr hoch aus.

\subsection{Ableitung von kontextspezifischen Integrationsprofilen}

Ein weiterer Schritt der Bestandsaufnahme der Integration von internem und externem Rechnungswesen bei deutschen Großunternehmen liegt in der Ableitung kontextspezifischer Integrationsprofile. Hierzu werden im Folgenden die bereits erläuterten Integrationsindizes in Abhängigkeit von unternehmensinternen bzw. umfeldbezogenen Einflussfaktoren ermittelt. Ziel dieser Vorgehensweise ist es, anhand von Mittelwertvergleichen aufzuzeigen, ob statistisch signifikante Zusammenhänge zwischen den besagten Einflussfaktoren und der jeweiligen Integrationsintensität innerhalb der verschiedenen Aktionsfelder bestehen.

Als erster Kontextfaktor wird danach differenziert, ob die Unternehmen der Hauptstichprobe börsennotiert sind oder nicht. Wie Abbildung 13 zeigt, ergibt der Mann-Whitney-U-Test ${ }^{346}$ hier einige statistisch (hoch) signifikante Unterschiede zwischen den Vergleichsgruppen. 


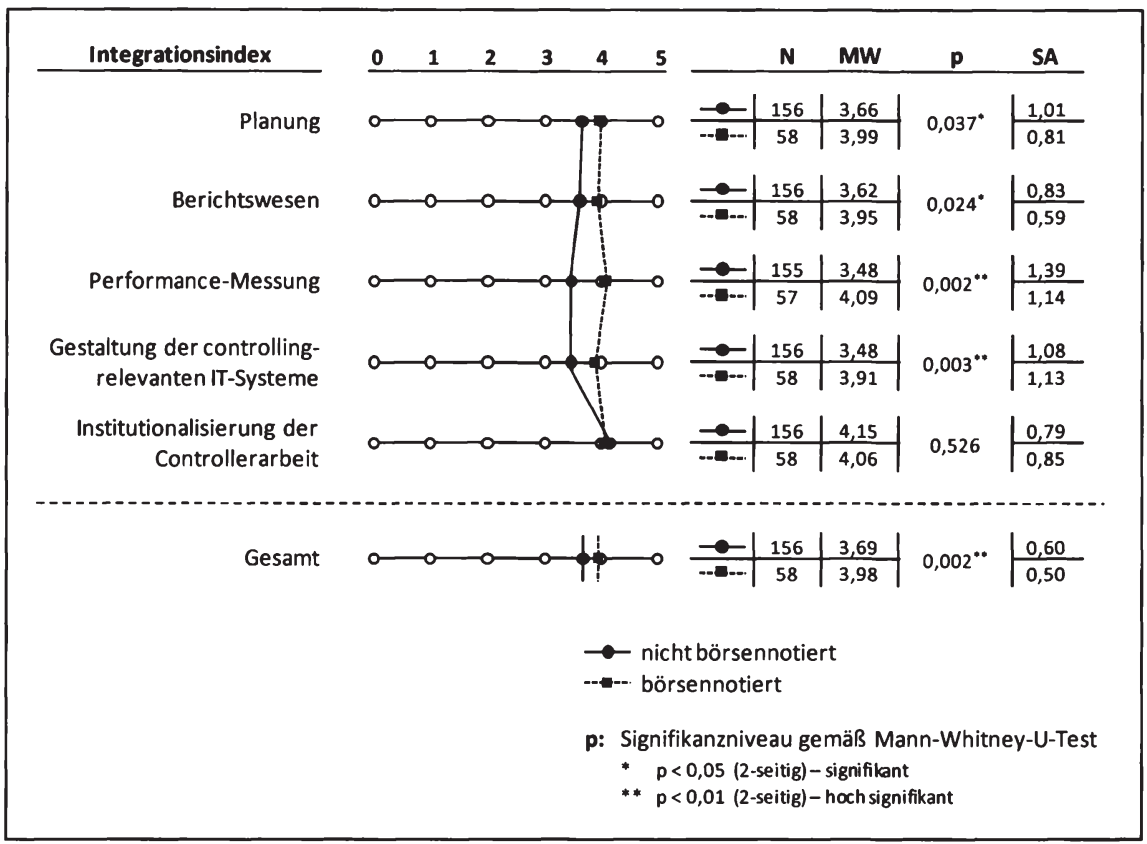

Abbildung 13: Integrationsprofil in Abhängigkeit der Börsennotierung

Die börsennotierten Unternehmen weisen mit einem Mittelwert in Höhe von 3,98 eine höhere Gesamtintegrationsintensität des Rechnungswesens auf als die nicht börsennotierten Unternehmen, deren Wert bei 3,69 liegt. Dieser statistisch hoch signifikante Unterschied lässt sich genauer analysieren, indem die einzelnen Aktionsfelder betrachtet werden. Hier zeigt sich, dass die börsennotierten Unternehmen erwartungsgemäß sowohl innerhalb der Planung und des Berichtswesens als auch insbesondere innerhalb der Performance-Messung und der Gestaltung der controllingrelevanten ITSysteme eine signifikant höhere Integrationsintensität aufweisen als es bei den nicht börsennotierten Unternehmen der Fall ist. Im Bereich der Institutionalisierung lässt sich hingegen diesbezüglich kein signifikanter Unterschied $(p=0,526)$ zwischen den beiden Vergleichsgruppen ermitteln. Offensichtlich ist der Kontextfaktor Börsennotierung somit kein ausschlaggebendes Kriterium im Hinblick auf die Integrationsintensität in diesem Bereich.

Ein möglicher Erklärungsansatz für das vorliegende Ergebnis liegt in den besonderen Anforderungen, denen börsennotierte Unternehmen unterliegen. Diese müssen die 
seitens der Investoren vorgegebenen Ziele in interne Pläne umsetzen - etwa in Form von Budgets für verschiedene Geschäftseinheiten -, was eine stärkere Integration im Bereich Planung erforderlich macht. ${ }^{347}$ Darüber hinaus ist aus Gründen der Verhaltenssteuerung eine Anbindung der Führungskräftevergütung an Erfolgsgrößen der externen Rechnungslegung sinnvoll. ${ }^{348}$ Die extern veröffentlichten Abschlussdaten müssen zudem seitens der Unternehmen gegenüber dem Kapitalmarkt betriebswirtschaftlich erläutert werden. Hierzu sind Informationen aus dem internen Berichtswesen notwendig, die wiederum im Einklang mit den Wertansätzen der externen Rechnungslegung stehen bzw. nachvollziehbar übergeleitet werden können müssen. ${ }^{349} \mathrm{Um}$ den umfangreichen Publikationsanforderungen - etwa in Form von Quartalsberichten - inhaltlich sowie zeitlich entsprechen zu können, ist zudem eine erhöhte Integration im Bereich der IT-Systeme erforderlich. ${ }^{350}$

Hingegen scheint sich die Frage der Börsennotierung nicht auf die Integrationsintensität des Rechnungswesens im Bereich der Aufbau- bzw. Ablauforganisation auszuwirken. Denkbar ist, dass hierfür zwei unterschiedliche Ursachen verantwortlich sind. So führt bei den börsennotierten Unternehmen der soeben aufgezeigte Abstimmungsbedarf zwischen Bilanzierung und Controlling zu einer verstärkten aufbau- und ablauforganisatorischen Integration. Die nicht börsennotierten Unternehmen weisen vor diesem Hintergrund zwar lediglich einen geringeren Integrationsbedarf auf. Sie sind jedoch im Vergleich zu den gelisteten Unternehmen signifikant kleiner, ${ }^{351}$ sodass sie im Bereich der Finanzfunktionen per se eine geringere Anzahl an Beschäftigten und folglich weniger komplexe bzw. spezialisierte Strukturen aufweisen dürften. ${ }^{352}$ Dieser Größeneffekt könnte dazu führen, dass auch bei den nicht börsennotierten Unternehmen beide Funktionsbereiche sowohl aufbau- als auch ablauforganisatorisch stark miteinander verzahnt sind. Die hohe Integration im Bereich der Institutionalisierung der Controllerarbeit ließe sich somit bei den hier gegenübergestellten Gruppen von Unternehmen auf jeweils unterschiedliche Ursachen zurückführen.

Vgl. Weißenberger (2007a), S. 196.

Vgl. Haller (1997b), S. 272.

Vgl. Weißenberger (2008a), Rz. 61.

Vgl. Fleischer (2005), S. 194f.

Dies ergab die Durchfuhrung des Mann-Whitney-U-Tests bezüglich der Kriterien Umsatz und Mitarbeiteranzahl (jeweils: $p=0,00$ ).

Vgl. Chenhall (2003), S. 22f. 
Gestützt werden diese Überlegungen durch das in Abbildung 14 dargestellte, unternehmensgrößenabhängige Integrationsprofil. Demnach weisen die als 'klein' eingestuften Unternehmen mit einem durchschnittlichen Wert von 3,59 eine hoch signifikant geringere Gesamtintegrationsintensität des Rechnungswesens auf als die 'großen' Unternehmen der Hauptstichprobe, deren Mittelwert bei 3,92 liegt. ${ }^{353}$

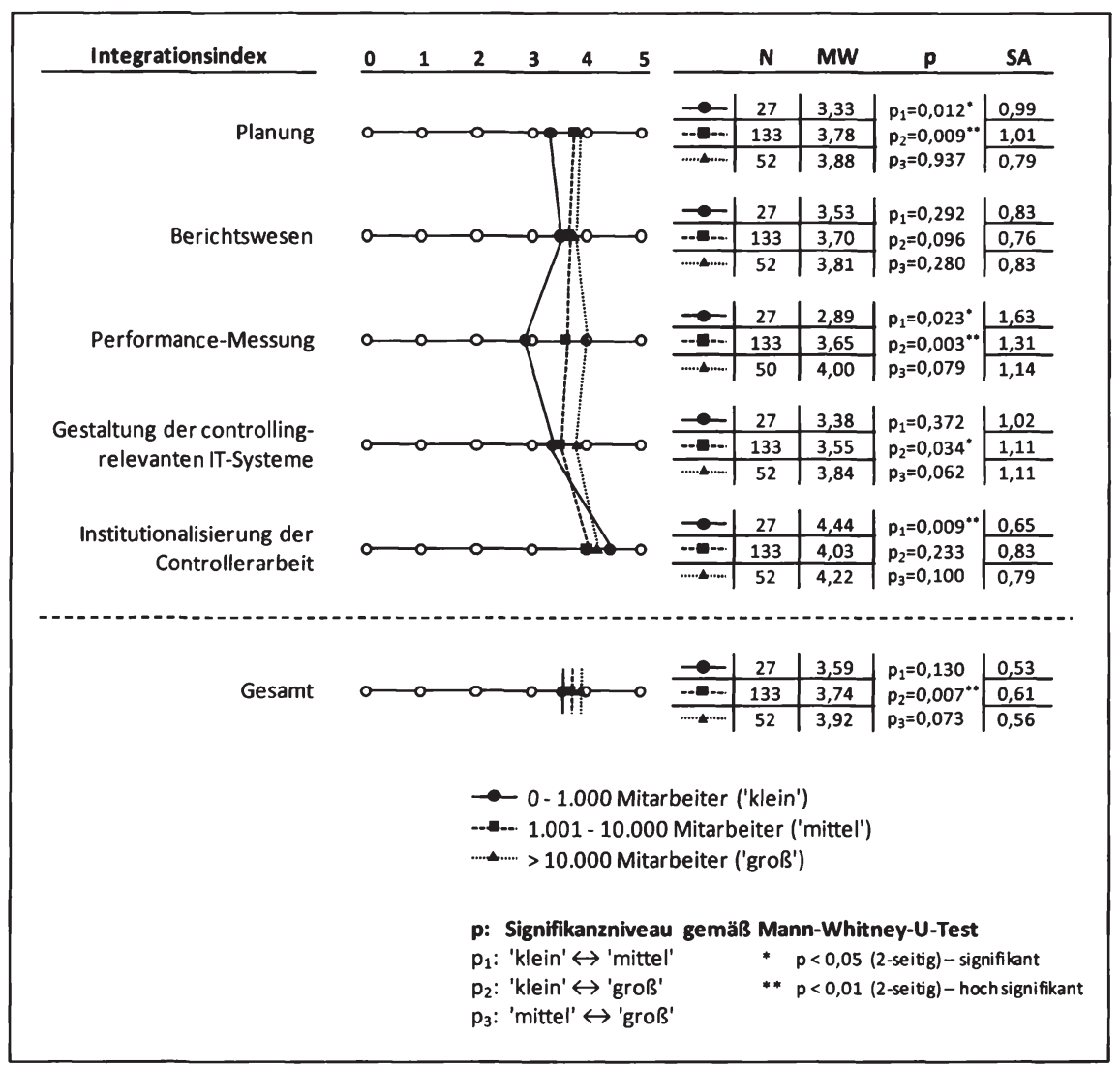

Abbildung 14: Integrationsprofil in Abhängigkeit der UnternehmensgröBe

353 In der empirischen Untersuchung von Müller (2006) weisen die Unternehmen des Clusters 'groß, international' zwar ebenfalls einen leicht höheren Harmonisierungsgrad als die 'kleinen, nationalen' Unternehmen auf, allerdings ist dieser Unterschied statistisch nicht signifikant, vgl. Müller (2006), S. 204. 
Wie der Blick auf die Integrationssubindizes deutlich macht, liegen innerhalb der ersten vier Aktionsfelder der Controllerarbeit ebenfalls entsprechende Mittelwertunterschiede zwischen den 'kleinen' und den 'großen' Unternehmen vor. ${ }^{354}$ Im Bereich der Institutionalisierung der Controllerarbeit hingegen zeigt sich der oben angesprochene Größeneffekt, da hier sowohl die 'kleinen' als auch die 'großen' Unternehmen eine hohe Integrationsintensität $(4,44$ bzw. 4,22) aufweisen, während Controlling und Bilanzierung bei den 'mittleren' Unternehmen am wenigsten stark aufbau- und ablauforganisatorisch verzahnt sind. Die durchschnittliche Integrationsintensität liegt in diesem Fall lediglich bei 4,03.

Ein ähnliches Muster wie im Falle des zuvor betrachteten Kriteriums der Börsennotierung resultiert aus der Ermittlung der Integrationsindizes in Abhängigkeit des jeweils führenden Rechnungslegungsstandards der Unternehmen (Leading GAAP). Wie sich Abbildung 15 entnehmen lässt, weisen die IFRS-Bilanzierer eine im Durchschnitt höhere Gesamtintegrationsintensität auf als die HGB-Bilanzierer (3,90 im Vergleich zu 3,56).

Auch dieser höchst signifikante Unterschied spiegelt sich in den ersten vier Aktionsfeldern wider, da sich hier ebenfalls entsprechende Abweichungen zwischen den beiden betrachteten Vergleichsgruppen ergeben. Wie bereits im Falle des Kontextfaktors Börsennotierung ergibt sich jedoch im Bereich der Institutionalisierung der Controllerarbeit erneut kein signifikanter Mittelwertunterschied $(p=0,794)$. Der verwendete Rechnungslegungsstandard stellt somit ebenfalls keinen bedeutsamen Einflussfaktor hinsichtlich der Integrationsintensität innerhalb dieses Aktionsfelds dar. ${ }^{355}$

Die festgestellte erhöhte Integrationsintensität der IFRS-Anwender gegenüber den nach HGB bilanzierenden Unternehmen insbesondere innerhalb der drei originären Aktionsfelder unterstützt aus empirischer Sicht die in der Literatur vielfach formulierte Aussage, dass die IFRS einen bedeutsamen Treiber für die Integration des Rechnungs-

354 Im Falle des Berichtswesens ist diese Abweichung mit einem p-Wert von 0,096 zwar aufgrund des im Rahmen der vorliegenden Arbeit verwendeten Signifikanzniveaus von $5 \%$ zunăchst als nicht signifikant einzustufen. Greift man für die Beurteilung jedoch auf ein - ebenfalls hăufig angesetztes - weniger strenges Signifikanzniveau von $10 \%$ zuruck, so ergibt sich auch hier ein signifikanter Mittelwertunterschied.

355 An dieser Stelle ist ein ăhnlicher Größeneffekt wie im Falle der Börsennotierung denkbar, da die HGB-Bilanzierer bezuglich der Kriterien Umsatz und Mitarbeiteranzahl eine signifikant geringere Unternehmensgröße aufweisen als die IFRS-Bilanzierer (jeweils: $p=0,00$ ). 
wesens darstellen, da sie aufgrund ihrer ökonomischen Fundierung aus konzeptioneller Sicht besser für Zwecke der internen Unternehmenssteuerung geeignet sind als die durch das Vorsichtsprinzip geprägten HGB-Vorschriften. ${ }^{356}$

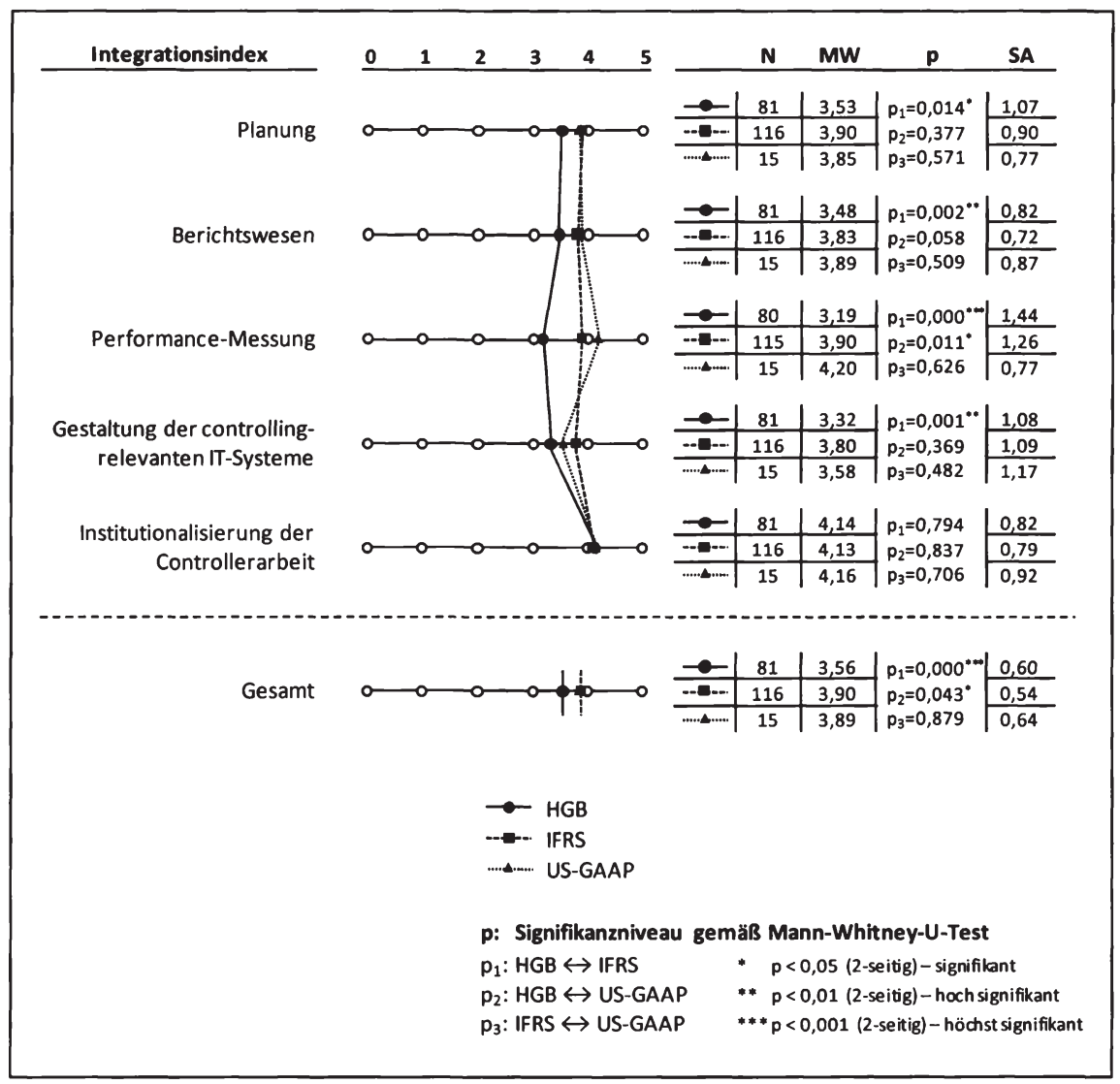

Abbildung 15: Integrationsprofil in Abhăngigkeit des fuhrenden Rechnungslegungsstandards

Der Vergleich von HGB- und US-GAAP-Anwendern fällt ähnlich aus wie der soeben dargestellte Fall. Die nach US-GAAP bilanzierenden Unternehmen der Hauptstichprobe weisen eine signifikant höhere Gesamtintegrationsintensität des Rechnungswesens $(3,89)$ als die HGB-Bilanzierer $(3,56)$ auf. Zwar offenbart der Blick auf 
die aktionsfeldspezifischen Integrationsindizes hier einige nicht signifikante Unterschiede zwischen den Vergleichsgruppen. Jedoch sind diese vor dem Hintergrund der geringen Fallzahl von US-GAAP-Anwendern zu relativieren. ${ }^{357}$ Dies gilt in gleicher Weise auch für das Nichtvorliegen signifikanter Abweichungen zwischen IFRS- und US-GAAP-Bilanzierern; verlässliche Aussagen sind an dieser Stelle nicht möglich.

Das soeben betrachtete Integrationsprofil steht im Einklang mit den Ergebnissen der empirischen Studie von Müller (2006), der sowohl für IFRS- als auch für USGAAP-Anwender einen signifikant höheren Harmonisierungsgrad ermittelt, als es bei den nach HGB bilanzierenden Unternehmen der Fall ist. ${ }^{358}$

Ein weiteres kontextspezifisches Integrationsprofil kann anhand der jeweiligen Hierarchiestufe der untersuchten Unternehmen innerhalb eines Konzernverbundes gebildet werden. Wie Abbildung 16 zeigt, ist zunächst zu konstatieren, dass es zwischen Spitzenholdings, Zwischenholdings und Tochterunternehmen zu keinerlei signifikanten Unterschieden hinsichtlich der Gesamtintegrationsintensität des Rechnungswesens kommt. Dies ist lediglich auf Aktionsfeldebene in den Bereichen Planung und Performance-Messung der Fall. In der Planung ergibt sich hinsichtlich der Subindexmittelwerte sowohl bei den Spitzenholdings $(3,90)$ als auch bei den Zwischenholdings $(3,85)$ eine signifikante Abweichung gegenüber den diesbezüglich schwächer integrierten Tochterunternehmen $(3,46)$. Zudem zeigt sich innerhalb der Performance-Messung bei den Tochterunternehmen mit einem Mittelwert von 3,27 ein signifikant geringeres Integrationsniveau als bei den Spitzenholdings $(3,86)$.

Das Ergebnis der nicht signifikant voneinander abweichenden Gesamtintegrationsintensität zwischen (Spitzen-)Holdings und Tochterunternehmen kann möglicherweise darauf zurückgeführt werden, dass seitens der Mutterunternehmen hinsichtlich grundsätzlicher Aspekte - wie der Ausgestaltung zentraler Managementberichte, der durchgängigen Implementierung von IT-Systemen oder der Organisation der Controller-

Die Tatsache, dass sich teilweise keine statistisch signifikanten Unterschiede zeigen, bedeutet hier nicht per se, dass diese nicht existent bzw. auszuschließen sind. Da empirische Effekte mit abnehmender Fallzahl immer stärker sein müssen, um überhaupt nachgewiesen werden zu können, kann hingegen unterstellt werden, dass die sich dennoch als signifikant erweisenden Unterschiede umso bedeutsamer sind, vgl. Janssen/Laatz (2007), S. 344f. 
arbeit - konzerninterne Vorgaben bzw. Richtlinien verabschiedet und durchgesetzt werden. ${ }^{359}$

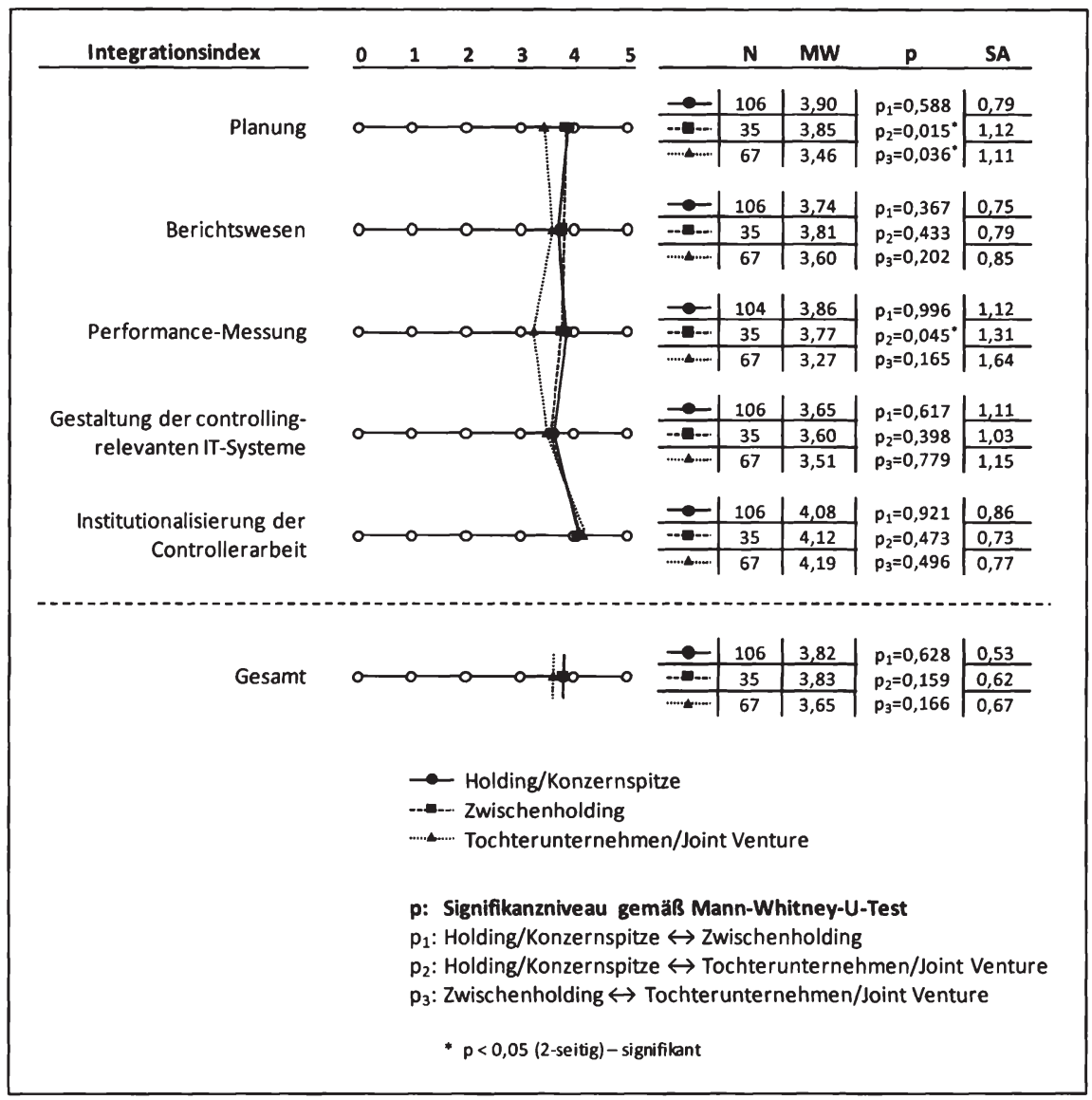

Abbildung 16: Integrationsprofil in Abhăngigkeit der Hierarchiestufe innerhalb eines Konzernverbundes

Wie die obigen empirischen Ergebnisse zudem zeigen, wird den Tochterunternehmen bei der Ausgestaltung der operativen Ergebnisplanung hingegen offensichtlich

359 Dies könnte beispielsweise durch Weisungen seitens des Zentralcontrollings umgesetzt werden, vgl. Simons/Weißenberger (2009b), S. 450. Empirische Ergebnisse zum Einfluss einer Einbindung in Konzernstrukturen auf die Integration des Rechnungswesens finden sich bei Jahnke/ Wielenberg/Schumacher (2007), S. 374f. 
mehr Freiheit zugestanden, von den Wertansätzen der externen Rechnungslegung abzuweichen und beispielsweise auch nichtfinanzielle Größen zu berücksichtigen. Statt für Zwecke der Performance-Messung - ähnlich wie die (Zwischen-)Holdings vermehrt auf Ergebnisgrößen der externen Finanzberichterstattung zurückzugreifen, können sie darüber hinaus die Entlohnung ihrer Führungskräfte ebenfalls stärker auf Basis anderer Größen, wie z.B. leistungswirtschaftlicher Kennzahlen, vornehmen. Da Tochterunternehmen im Vergleich $\mathrm{zu}$ (Zwischen-)Holdings hinsichtlich ihrer Geschäftstätigkeit als tendenziell operativer geprägt anzusehen sind, wird hierdurch den für diese Unternehmen existierenden Steuerungserfordernissen Rechnung getragen. ${ }^{360}$

Unterscheidet man zwischen Unternehmen, die einer geringen Volatilität des operativen Ergebnisses unterliegen, und Unternehmen, die diesbezüglich eine hohe Volatilität aufweisen, so ergibt sich das in Abbildung 17 dargestellte Integrationsprofil. ${ }^{361}$ Die Unternehmen mit geringer Ergebnisvolatilität zeichnen sich bei einem durchschnittlichen Gesamtintegrationsindex von 3,84 durch ein signifikant höheres Gesamtintegrationsniveau aus als die Vergleichsgruppe mit hoher Ergebnisvolatilität, deren Wert bei 3,63 liegt. Getrieben wird diese Abweichung - dies zeigt sich bei Betrachtung der einzelnen aktionsfeldbezogenen Integrationssubindizes - durch die Planung. Während innerhalb der übrigen vier Bereiche keinerlei signifikante Abweichungen zu verzeichnen sind, liegt hier ein statistisch hoch signifikanter Unterschied hinsichtlich der Integrationsintensität in Abhängigkeit des betrachteten Kontextfaktors vor. So weisen die Unternehmen mit geringer Ergebnisvolatilität innerhalb der Planung ein durchschnittliches Integrationsniveau von 3,90 auf, wohingegen der Wert im Falle hoher Ergebnisvolatilität nur bei 3,50 liegt.

Das vorliegende Ergebnis kann dahingehend erklärt werden, dass die Unternehmen im Falle hoher Ergebnisvolatilität zu Planungszwecken nur in vergleichsweise geringerem Ausmaß auf Wertansätze der externen Rechnungslegung zurückgreifen und vermehrt kalkulatorische Größen verwenden, um sich hierdurch eine höhere

Vgl. Euske/Lebas/McNair (1993), S. 287-289, Schweitzer (2003), S. 440ff. sowie Weißenberger/ Angelkort (2007a), S. 430.

Die Unterscheidung in die beiden Kategorien wurde anhand der Stellungnahmen der Unternehmen bezüglich der Aussage „Die Volatilităt unseres operativen Unternehmensergebnisses ist sehr hoch." vorgenommen. Diejenigen Unternehmen, die dieser Aussage auf der verwendeten 6stufigen Rating-Skala nicht zugestimmt haben (Antwortwerte 0 bis 2), wurden der Kategorie 'geringe Volatilităt des operativen Ergebnisses' zugeordnet. Die der Aussage zustimmenden Unternehmen (Antwortwerte 3 bis 5) bilden die Kategorie 'hohe Volatilităt des operativen Ergebnisses'. 
Flexibilität im Planungsprozess zu erhalten. So wäre es im Falle stark schwankender Rohstoffpreise beispielsweise denkbar, innerhalb der Ergebnisplanung den geplanten Rohstoffverbrauch nicht auf Basis der - ohnehin nicht beeinflussbaren - erwarteten Marktpreise, sondern anhand von Standardkosten zu bewerten. Hierdurch könnten die Unternehmen eine gewisse Stetigkeit und somit Vergleichbarkeit bzw. Analysefähigkeit der Plandaten erzielen. ${ }^{362} \mathrm{Da}$ es im Rahmen des Reportings ohnehin regelmäßig zu Plan-Ist-Abweichungen kommt, kann der Effekt des Bewertungsunterschiedes ähnlich wie bei Währungsschwankungen - in Form einer Überleitungsrechnung transparent gemacht werden.

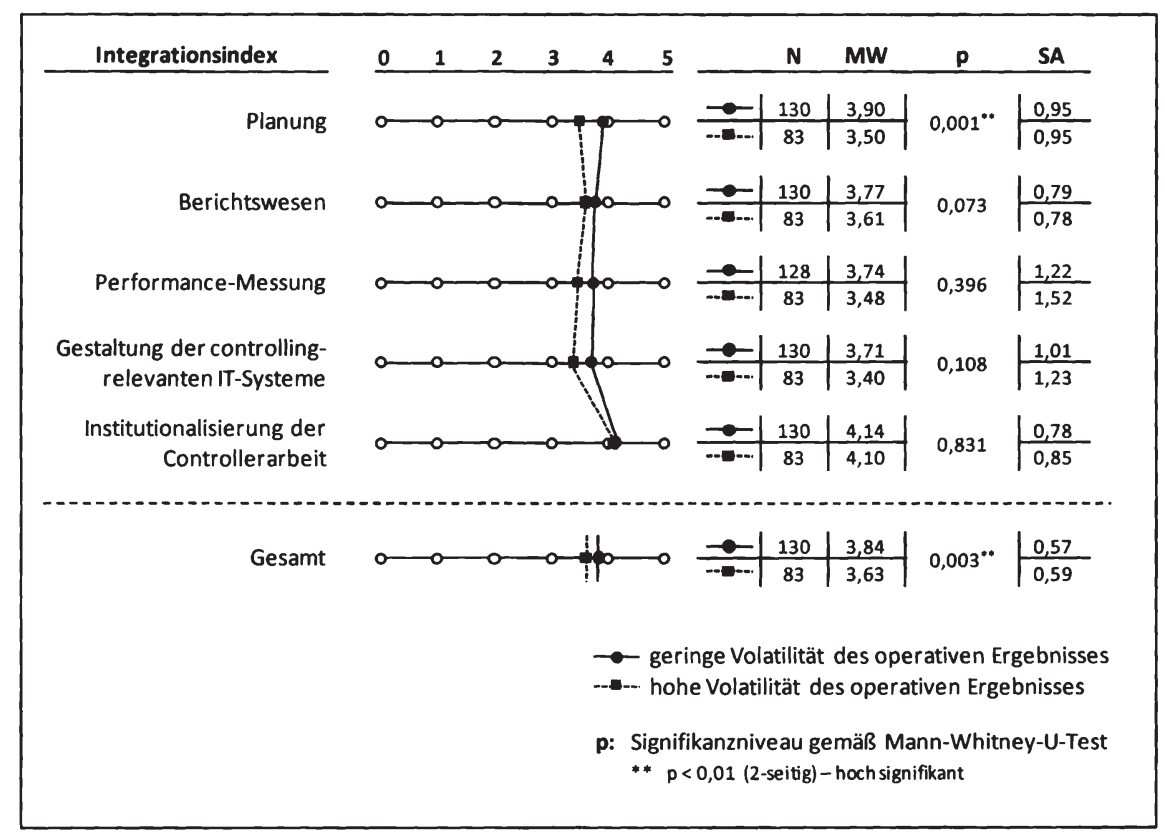

Abbildung 17: Integrationsprofil in Abhăngigkeit der Volatilität des operativen Ergebnisses

Die nun im letzten Schritt in die Analyse einbezogene Dynamik des Unternehmensumfelds stellt den einzigen im Rahmen dieser Arbeit betrachteten Kontextfaktor dar, in dessen Abhängigkeit es bei den untersuchten Unternehmen zu einem signifi-

362 Hingegen erschwert eine verstärkte Fair Value-Bilanzierung - wie etwa unter IFRS - die Planbzw. Vergleichbarkeit der Ergebnisse, vgl. Haeger (2006), S. 256 sowie Horváth/Pötsch (2008), S. 708. 
kanten Unterschied hinsichtlich der Integrationsintensität im Bereich Institutionalisierung der Controllerarbeit kommt. Zwar zeigt sich in dem in Abbildung 18 dargestellten Integrationsprofil - bedingt durch die ersten vier Aktionsfelder - hinsichtlich der Gesamtintegrationsintensität keine solche Abweichung zwischen den beiden Vergleichsgruppen. Jedoch weisen diejenigen Unternehmen, die in einem sich schnell ändernden Umfeld agieren, ${ }^{363}$ mit einem Mittelwert in Höhe von 4,27 ein höheres Integrationsniveau des Rechnungswesens innerhalb der Institutionalisierung der Controllerarbeit auf als die Unternehmen, die sich nicht in einem solchen Umfeld bewegen $(3,93)$.

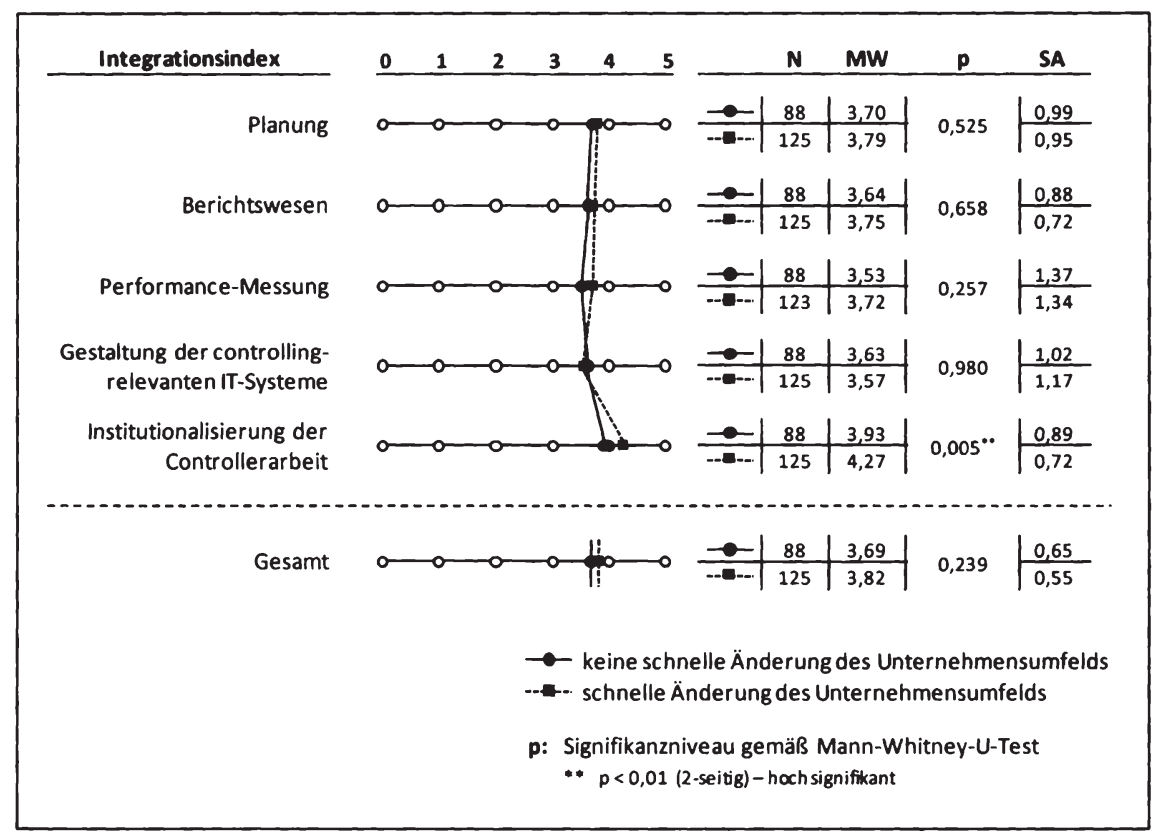

Abbildung 18: Integrationsprofil in Abhăngigkeit der Änderungsdynamik des Unternehmensumfelds

Die Unterscheidung in die beiden Kategorien wurde anhand der Stellungnahmen der Unternehmen bezüglich der Aussage „Das Umfeld, in dem unser Unternehmen agiert, ändert sich sehr schnell." vorgenommen. Diejenigen Unternehmen, die dieser Aussage auf der verwendeten 6-stufigen Rating-Skala nicht zugestimmt haben (Antwortwerte 0 bis 2), wurden der Kategorie 'keine schnelle Änderung des Unternehmensumfelds' zugeordnet. Die der Aussage zustimmenden Unternehmen (Antwortwerte 3 bis 5) bilden die Kategorie 'schnelle Änderung des Unternehmensumfelds'. 
Dieser statistisch hoch signifikante Unterschied kann derart interpretiert werden, dass die Unternehmen für die notwendigen institutionellen Rahmenbedingungen der Controllerarbeit sorgen, damit die mit einer hohen Änderungsdynamik einhergehenden Anforderungen erfüllt bzw. Herausforderungen bewältigt werden können. Da die aufbauorganisatorische Gestaltung von Controlling und Bilanzierung bei den untersuchten Unternehmen recht einheitlich ausgeprägt ist, ergibt sich der Integrationsunterschied zwischen den beiden Vergleichsgruppen offensichtlich in erster Linie aus den beiden ablauforganisatorischen Aspekten, die den fachlichen Austausch zwischen Controlling und Bilanzierung erfassen. ${ }^{364}$ Beispielsweise könnte sich eine hohe Änderungsdynamik des Unternehmensumfelds in schnell aufeinander folgenden technologischen Innovationen oder deutlichen Zinssatzänderungen am Finanzmarkt manifestieren. Beide Aspekte würden Indikatoren für eine möglicherweise notwendige außerplanmäßige Abschreibung im Rahmen eines Goodwill Impairment Tests darstellen. ${ }^{365}$ In einer solchen Situation wäre eine intensive Kommunikation zwischen Bilanzierung und Controlling erforderlich, etwa um die Auswirkungen der Umfeldänderungen auf die Berechnung des Nutzungswerts (value in use) einer zahlungsmittelgenerierenden Einheit (cash-generating unit) zu erörtern. ${ }^{366}$

Vgl. hierzu die Ausfuhrungen in Abschnitt D1.5 der vorliegenden Arbeit.

Vgl. Buhleier (2008), S. 459 sowie Haas (2009), S. $113 \mathrm{ff}$. (2009), S. $181 \mathrm{ff}$. 


\section{Stand der Umsetzung des Musters einer partiellen Integration des Rechnungswesens}

\subsection{Identifizierung und Quantifizierung des Musters einer partiel- len Integration des Rechnungswesens}

Die im Rahmen der bisherigen Analyse insgesamt festgestellte starke Integrationsintensität des internen und externen Rechnungswesens bezog sich auf die jeweils oberste Hierarchieebene der untersuchten Unternehmen. ${ }^{367}$ Da aus konzeptioneller Sicht das Muster einer partiellen Integration des Rechnungswesens, welches unterschiedliche Integrationsintensitäten innerhalb der verschiedenen Hierarchieebenen eines Unternehmens vorsieht, vorteilhaft erscheint, ${ }^{368}$ muss für eine umfassende Status-quoErmittlung zusätzlich eine hierarchiebezogene Analyse der Integration des Rechnungswesens vorgenommen werden. Daher erfolgt im nächsten Schritt die Überprüfung, inwieweit sich das Muster einer partiellen Integration bei den Unternehmen der Hauptstichprobe identifizieren lässt. Das Fragebogendesign für eine solche Analyse ist sehr aufwändig, da für jeden zu untersuchenden Aspekt Informationen bezüglich jeder der verschiedenen Hierarchiestufen erhoben werden müssen. Zudem ist nicht jeder der in Abschnitt D1 auf Gesamtunternehmensebene erhobenen Aspekte auch für eine nach Hierarchiestufen differenzierende Analyse geeignet. ${ }^{369}$ Daher erfolgt die Bestandsaufnahme des Musters einer partiellen Integration des Rechnungswesens lediglich exemplarisch für die Fragestellung der operativen Ergebnisplanung.

Die im Rahmen der Ermittlung der Integrationsintensität im Bereich Planung bereits behandelte Frage nach der Verwendung von Wertansätzen der externen Rechnungslegung für Zwecke der operativen Ergebnisplanung ${ }^{370}$ wird an dieser Stelle nicht mehr nur aus Gesamtunternehmenssicht, sondern auch auf den hierarchisch niedrige-

Die Unternehmensvertreter wurden am Anfang des Fragebogens gebeten, sămtliche Fragen wenn nicht anders angegeben - aus Gesamtunternehmenssicht zu beantworten und dabei ggf. existierende ubergeordnete Mutterunternehmen im Rahmen der Beantwortung nicht zu berücksichtigen.

Vgl. hierzu die Ausfuhrungen in Abschnitt B3.2 der vorliegenden Arbeit.

So könnte beispielsweise die Frage bezulglich der Überleitbarkeit des internen Ergebnisses auf das externe Ergebnis der GuV auf operativer Produkt- bzw. Prozessebene nicht beantwortet werden, da für diese Hierarchiestufe (z.B. ein einzelnes produzierendes Werk) aufgrund des hohen Aggregationsniveaus innerhalb der externen Finanzberichterstattung kein korrespondierendes Ergebnis separat ausgewiesen wird.

Vgl. hierzu Abschnitt D1.1 der vorliegenden Arbeit. 
ren Ebenen bis hin zur operativen Produkt- bzw. Prozessebene betrachtet. Wie Abbildung 19 zeigt, weisen die untersuchten Unternehmen mit abnehmenden Hierarchiestufen eine sinkende durchschnittliche Integrationsintensität des internen und externen Rechnungswesens hinsichtlich der operativen Ergebnisplanung auf.

Während auf Gesamtunternehmens- bzw. Konzernebene bei einer durchschnittlichen Integrationsintensität von 4,02 gut drei Viertel $(78,04 \%)$ der befragten Controller eine starke bzw. sehr starke Integration konstatieren, ist dies auf Segment-/ Geschäftsbereichs- bzw. Geschäftsfeldebene bei einem Mittelwert von 3,66 noch immerhin bei knapp zwei Dritteln $(65,66 \%)$ der Unternehmen der Fall. Ein noch deutlicherer Rückgang des Integrationsniveaus zeigt sich bei Betrachtung der Profit Center-Ebene sowie der operativen Produkt- und Prozessebene. Hier geben bei Mittelwerten von 3,03 bzw. 2,39 nur noch $43,35 \%$ bzw. $29,89 \%$ der Unternehmen an, im Bereich der operativen Ergebnisplanung stark bis sehr stark integriert zu sein. Im letzteren Fall der operativen Produkt- und Prozessebene weist bereits hingegen mit gut einem Drittel (33,91\%) ein größerer Anteil der Unternehmen eine nur sehr schwache bis schwache Integrationsintensität auf.

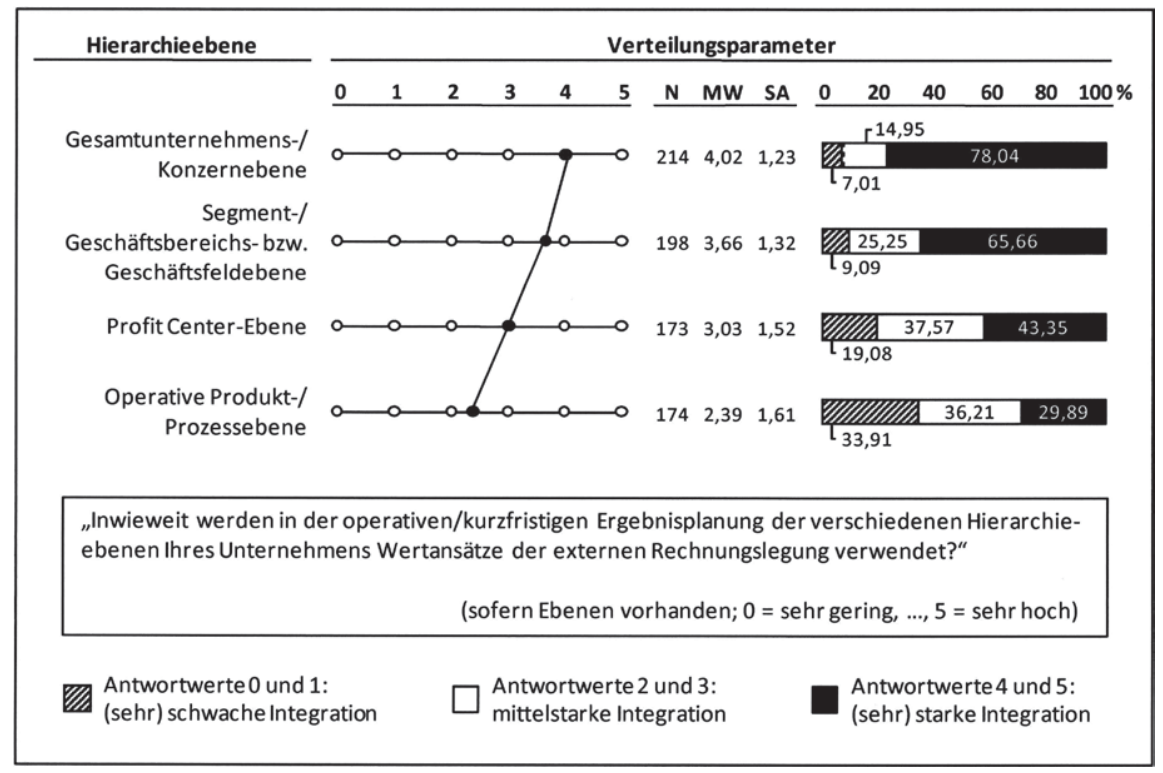

Abbildung 19: Hinweise auf eine partielle Integration des Rechnungswesens innerhalb der operativen Ergebnisplanung 
Zwar liefern die dargestellten Ergebnisse erste Hinweise auf das Vorliegen einer partiellen Integration des Rechnungswesens. Jedoch sind an dieser Stelle aufgrund des mit der Verwendung von Mittelwerten bzw. relativen Häufigkeiten einhergehenden hohen Aggregationsniveaus zunächst keine detaillierten Aussagen möglich. Die wenigen empirischen Untersuchungen, die sich bisher mit dem Muster einer partiellen Integration beschäftigt haben, sind über vergleichbar grobe (weil aggregierte) Betrachtungsweisen nicht hinausgegangen. ${ }^{371}$

In den oben dargestellten Ergebnissen zeigt sich, dass das Ausmaß der Standardabweichungen innerhalb der Antworten mit sinkender Hierarchieebene steigt. Hierin ist ein Indiz dafür zu sehen, dass bei den untersuchten Unternehmen unterschiedliche Integrationsmuster im Bereich der operativen Ergebnisplanung vorliegen. Diese sollen im Rahmen der nun folgenden Detailanalyse identifiziert werden.

Da sich unterschiedliche Ausgestaltungsformen der Integration des Rechnungswesens am deutlichsten im Zuge eines Vergleichs der hierarchisch höchsten mit der hierarchisch niedrigsten Ebene manifestieren, werden zu Analysezwecken ausschließlich die Antworten bezüglich der Gesamtunternehmens- bzw. Konzernebene einerseits und der Produkt- bzw. Prozessebene andererseits in Form einer Matrix gegenübergestellt. In Tabelle 12 kann zunächst für jede mögliche Antwortkombination die sich ergebende Fallzahl abgelesen werden. Darüber hinaus wurde hier eine Unterteilung der Matrix in fünf verschiedene Bereiche vorgenommen, die sich folgendermaßen beschreiben lassen:

- Der obere linke Bereich zeichnet sich durch eine einheitlich schwache Integration aus, d.h. keiner der Antwortwerte ist größer, mindestens einer aber kleiner als 2 .

- Der mittlere Bereich weist eine einheitlich mittelstarke Integration auf, d.h. die Antwortwerte liegen auf beiden betrachteten Ebenen entweder bei 2 oder 3 .

- Der untere rechte Bereich ist durch eine einheitlich starke Integration gekennzeichnet, d.h. keiner der Antwortwerte ist kleiner, mindestens einer aber größer als 3. 
- Der untere linke Bereich zeichnet sich durch eine partielle Integration aus, d.h. der Antwortwert auf Gesamtunternehmensebene beträgt mindestens 3 und ist zugleich mindestens um den Betrag von 2 höher als der Antwortwert auf Produkt- bzw. Prozessebene, welcher zugleich nicht höher als 2 sein darf.

- Der obere rechte Bereich bildet die Situation ab, dass der Antwortwert auf Produkt- bzw. Prozessebene mindestens 3 beträgt und zugleich mindestens um den Betrag von 2 höher ist als der Antwortwert auf Gesamtunternehmens- bzw. Konzernebene, welcher zugleich nicht höher als 2 sein darf.

\begin{tabular}{|c|c|c|c|c|c|c|c|}
\hline \multirow{2}{*}{\multicolumn{2}{|c|}{$\begin{array}{l}\text { Ausmaß der Verwendung von } \\
\text { Wertansätzen der externen } \\
\text { Rechnungslegung in der } \\
\text { operativen/kurzfristigen } \\
\text { Ergebnisplanung } \\
(0=\text { sehr gering, ...., } 5 \text { = sehr hoch } / \\
\qquad N=174)\end{array}$}} & \multicolumn{6}{|c|}{ Produkt-/Prozessebene } \\
\hline & & 0 & 1 & 2 & 3 & 4 & 5 \\
\hline \multirow{6}{*}{$\begin{array}{l}\text { Gesamtunternehmens-/ } \\
\text { Konzernebene }\end{array}$} & 0 & 3 & 0 & 0 & 0 & 0 & 0 \\
\hline & 1 & 1 & 7 & 0 & 1 & 0 & 0 \\
\hline & 2 & 1 & 0 & 2 & 0 & 0 & 0 \\
\hline & 3 & 6 & 2 & 3 & 9 & 0 & 0 \\
\hline & 4 & 11 & 11 & 9 & 10 & 19 & 1 \\
\hline & 5 & 5 & 12 & 18 & 11 & 14 & 18 \\
\hline
\end{tabular}

Tabelle 12: Ermittlung von Integrationsmustern innerhalb der operativen Ergebnisplanung

Da der letztgenannte Bereich oben rechts in der Matrix aus konzeptioneller Sicht nicht sinnvoll erscheint, beschränkt sich die weitere Analyse auf die vier übrigen 
Bereiche, ${ }^{372}$ wobei die Unternehmen mit einheitlich schwacher und einheitlich mittelstarker Integration $\mathrm{zu}$ einem gemeinsamen Integrationsmuster zusammengefasst werden. Auf Basis der oben beschriebenen Vorgehensweise lassen sich bei den Unternehmen der Hauptstichprobe folglich die drei in Abbildung 20 aufgeführten und quantifizierten Integrationsmuster identifizieren.

\begin{tabular}{|l|l|l|l|}
\hline Integrationsmusterinnerhalb der operativen Ergebnisplanung (N=173) & $\mathbf{N}$ & in\% \\
\hline $\begin{array}{l}\text { Einheitlich schwach bzw. } \\
\text { mittelstark integriert }\end{array}$ & 26 & 15,03 \\
\hline Einheitlich stark integriert & 73 & 42,20 \\
\hline & Partiellintegriert & 74 & 42,78 \\
\hline
\end{tabular}

Abbildung 20: Identifixierte Integrationsmuster innerhalb der operativen Ergebnisplanung

Es zeigt sich nun - im Gegensatz zur Mittelwertbetrachtung in Abbildung 19 -, dass die partielle Integration sowie die einheitlich starke Integration im Rahmen der operativen Ergebnisplanung mit 42,78\% bzw. 42,20\% die beiden dominierenden Integrationsmuster bilden. Eine einheitlich schwache bzw. einheitlich mittelstarke Integration des Rechnungswesens weisen hingegen lediglich 15,03\% der untersuchten Unternehmen auf.

372 Unterstutzt wird diese Schlussfolgerung durch die Tatsache, dass bis auf eine Ausnahme keines der Unternehmen in dem genannten Bereich liegt. Da ein einzelnes Unternehmen als nicht bedeutsam einzustufen ist - die Vermutung liegt nahe, dass es sich hier eventuell sogar um eine fehlerhafte Beantwortung der Fragestellung handelt -, ist die Eliminierung dieses Falles vertretbar. 


\subsection{Einfluss von Kontextfaktoren auf die Umsetzung unterschied- licher Integrationsmuster}

Nachdem die Identifizierung und Quantifizierung der drei verschiedenen Integrationsmuster erfolgt ist, soll im nächsten Schritt untersucht werden, welche Einflussfaktoren mit der Anwendung dieser Muster seitens der Unternehmen in Zusammenhang stehen. Hierzu wird zunächst unter Zuhilfenahme des Kriteriums der Börsennotierung die in Tabelle 13 dargestellte Kreuztabelle ${ }^{373}$ gebildet.

\begin{tabular}{|crr|r|r|r|}
\hline & & \multicolumn{2}{|c|}{ Börsennotierung } & \multirow{2}{*}{ Gesamt } \\
\cline { 4 - 5 } & & \multicolumn{2}{|c|}{ Nein } & \multicolumn{1}{|c|}{ Ja } & \\
\hline Integrationsmuster & Einheitlich schwach bzw. & & & \\
& mittelstark integriert & Anzahl & 25 & 1 & 26 \\
& & $\%$ & $96,15 \%$ & $3,85 \%$ & $100 \%$ \\
\cline { 2 - 5 } & Einheitlich stark integriert & Anzahl & 51 & 22 & 73 \\
& & $\%$ & $69,86 \%$ & $30,14 \%$ & $100 \%$ \\
\cline { 2 - 5 } & Partiell integriert & Anzahl & 49 & 25 & 74 \\
& & $\%$ & $66,22 \%$ & $33,78 \%$ & $100 \%$ \\
\hline Gesamt & Anzahl & 125 & 48 & 173 \\
& & $\%$ & $72,25 \%$ & $27,75 \%$ & $100 \%$ \\
\hline
\end{tabular}

Tabelle 13: Kreuztabelle Integrationsmuster und Börsennotierung

Die Gruppe der nicht börsennotierten Unternehmen, die hier insgesamt einen Anteil von 72,25\% ausmacht, stellt einen überdurchschnittlich hohen Anteil (96,15\%) der einheitlich schwach bzw. einheitlich mittelstark integrierten Unternehmen. Gleichzeitig fällt auf diese Unternehmen jeweils nur ein unterdurchschnittlicher Anteil der einheitlich stark $(69,86 \%)$ und der partiell integrierten Unternehmen $(66,22 \%)$. Hingegen stellen die börsennotierten Unternehmen, die hier insgesamt einen Anteil von 27,75\% ausmachen, einen unterdurchschnittlichen Anteil (3,85\%) der einheitlich schwach bzw. einheitlich mittelstark integrierten Unternehmen und einen überdurchschnittlichen Anteil sowohl der einheitlich stark (30,14\%) als auch der partiell integrierten Unternehmen $(33,78 \%)$.

Offensichtlich besteht somit ein Zusammenhang zwischen dem Kriterium einer möglichen Börsennotierung und der Anwendung der Integrationsmuster seitens der

373 Vgl. zu den diesbezüglichen methodischen Grundlagen die Ausführungen in Abschnitt C3 der vorliegenden Arbeit. 
Unternehmen, dessen Signifikanz durch das in Tabelle 14 dargestellte Ergebnis des Chi-Ouadrat-Tests bestätigt wird. ${ }^{374}$

\begin{tabular}{|l|c|c|c|}
\hline & Wert & df & $\begin{array}{r}\text { Asymptotische Signifikanz } \\
\text { (2-seitig) }\end{array}$ \\
\hline $\begin{array}{l}\text { Chi-Quadrat nach Pearson } \\
\text { Anzahl der gültigen Fälle }\end{array}$ & $\begin{array}{r}8,962^{\text {a) }} \\
173\end{array}$ & 2 & 0,011 \\
\hline
\end{tabular}

a) 0 Zellen (0\%) haben eine erwartete Häufigkeit kleiner 5. Die minimale erwartete Häufigkeit ist 7,21.

Tabelle 14: Chi-Quadrat-Test fur Kreuztabelle Integrationsmuster und Borsennotierung

Als wichtiges Zwischenergebnis kann zudem konstatiert werden, dass sich die börsennotierten Unternehmen der Hauptstichprobe fast ausschließlich (47 von $48=$ 97,92\%) auf die Anwendung des Musters einer einheitlich starken bzw. einer partiellen Integration des Rechnungswesens im Bereich der operativen Ergebnisplanung beschränken, während für die nicht börsennotierten Unternehmen offensichtlich auch das Muster einer einheitlich schwachen bzw. einheitlich mittelstarken Integration relevant ist $(25$ von $125=20,00 \%)$.

Der zu konstatierende Zusammenhang zwischen Börsennotierung und Integrationsmuster liefert somit einen weiteren Hinweis darauf, dass insbesondere börsennotierte Unternehmen auf eine erhöhte Integration - sei sie einheitlich stark oder partiell - angewiesen sind, um die besonderen Anforderungen im Rahmen der Investorenkommunikation erfüllen zu können. ${ }^{375}$ So wird durch die Integration beispielsweise ermöglicht, seitens der Investoren aufgestellte Zielvorgaben im Rahmen der Ergebnisplanung in Budgets für die einzelnen Unternehmensbereiche umzusetzen.

Als weiterer Einflussfaktor hinsichtlich der Anwendung der unterschiedlichen Integrationsmuster wird der bei den Unternehmen führende Rechnungslegungsstandard (Leading GAAP) betrachtet und in analoger Weise zur Bildung der in Tabelle 15 dargestellten Kreuztabelle herangezogen. ${ }^{376}$

Für die Durchfuhrung des Chi-Quadrat-Tests wurde auf die Software SPSS 17.0 zurückgegriffen. schnitt D2.3 der vorliegenden Arbeit.

376 Die Analyse der übrigen in Abschnitt D2.3 zur Bildung der Integrationsprofile verwendeten Kontextfaktoren (Hierarchiestufe innerhalb eines Konzernverbunds, Volatilităt des operativen Ergebnisses, Änderungsdynamik des Unternehmensumfelds) ergab keinerlei signifikante Zusammenhănge zur Umsetzung des Musters einer partiellen Integration des Rechnungswesens innerhalb der operativen Ergebnisplanung. 


\begin{tabular}{|clr|r|r|r|r|}
\hline & & & \multicolumn{3}{|c|}{ Leading GAAP } & \multirow{2}{*}{ Gesamt } \\
\cline { 4 - 6 } $\begin{array}{c}n n n y y n \\
\text { Integrations- } \\
\text { muster }\end{array}$ & Einheitlich schwach bzw. & HGB & IFRS & US-GAAP & \\
& mittelstark integriert & Anzahl & 19 & 6 & & \\
& & $\%$ & $73,08 \%$ & $23,08 \%$ & $3,85 \%$ & $100 \%$ \\
\cline { 2 - 6 } & Einheitlich stark integriert & Anzahl & 20 & 46 & 6 & 72 \\
& & $\%$ & $27,78 \%$ & $63,89 \%$ & $8,33 \%$ & $100 \%$ \\
\cline { 2 - 6 } & Partiell integriert & Anzahl & 23 & 45 & 5 & 73 \\
& & $\%$ & $31,51 \%$ & $61,64 \%$ & $6,85 \%$ & $100 \%$ \\
\hline Gesamt & Anzahl & 62 & 97 & 12 & 171 \\
& & $\%$ & $36,26 \%$ & $56,73 \%$ & $7,02 \%$ & $100 \%$ \\
\hline
\end{tabular}

Tabelle 15: Kreuztabelle Integrationsmuster und Leading GAAP

Die Analyse ergibt auch hier ein ähnliches Bild wie zuvor im Falle des Kriteriums der Börsennotierung. Obwohl die Gruppe der HGB-Anwender hier insgesamt nur einen Anteil von 36,26\% ausmacht, stellt sie einen überdurchschnittlich hohen Anteil (73,08\%) der einheitlich schwach bzw. einheitlich mittelstark integrierten Unternehmen. Gleichzeitig fällt auf diese Unternehmen jeweils nur ein unterdurchschnittlicher Anteil der einheitlich stark $(27,78 \%)$ und der partiell integrierten Unternehmen (31,51\%). Die IFRS-Anwender, die hier insgesamt einen Anteil von 56,73\% ausmachen, stellen dagegen einen unterdurchschnittlichen Anteil $(23,08 \%)$ der einheitlich schwach bzw. einheitlich mittelstark integrierten Unternehmen sowie einen überdurchschnittlichen Anteil sowohl der einheitlich stark $(63,89 \%)$ als auch der partiell integrierten Unternehmen (61,64\%). ${ }^{377}$

Der festgestellte Zusammenhang zwischen dem Kriterium des führenden Rechnungslegungsstandards und der Anwendung der Integrationsmuster seitens der Unternehmen ist - wie das in Tabelle 16 dargestellte Ergebnis des Chi-Quadrat-Tests belegt - statistisch hoch signifikant.

\begin{tabular}{|l|c|c|c|}
\hline & Wert & df & $\begin{array}{r}\text { Asymptotische Signifikanz } \\
\text { (2-seitig) }\end{array}$ \\
\hline $\begin{array}{l}\text { Chi-Quadrat nach Pearson } \\
\text { Anzahl der gültigen Fälle }\end{array}$ & $\begin{array}{r}18,309^{\text {a) }} \\
171\end{array}$ & 4 & 0,001 \\
\hline
\end{tabular}

a) 1 Zelle $(11,1 \%)$ hat eine erwartete $\overline{H a ̈ u f i g k e i t}$ kleiner 5 . Die minimale erwartete Häufigkeit ist $1,82$.

Tabelle 16: Chi-Quadrat-Test für Kreuztabelle Integrationsmuster und Leading GAAP

377 Tendenziell ergibt sich bei den US-GAAP-Anwendern ein ähnliches Bild wie bei den IFRSBilanzierern; aufgrund der geringen Fallzahl wird dieser Aspekt aber nicht weiter erörtert. 
Zudem ist festzuhalten, dass zwar knapp ein Drittel (19 von $62=30,65 \%)$ der HGB-Anwender der Hauptstichprobe, aber nur 6,19\% (=6 von 97) der IFRSAnwender einheitlich schwach bzw. einheitlich mittelstark integriert sind. Hingegen sind 93,81\% (= 91 von 97) der IFRS-Anwender, jedoch lediglich gut zwei Drittel (43 von $62=69,35 \%$ ) der HGB-Anwender einheitlich stark oder partiell integriert.

Es zeigt sich anhand der vorliegenden Ergebnisse der empirischen Analyse somit erneut, dass die Anwendung der IFRS seitens der Unternehmen einen wichtigen Treiber für die Integration des Rechnungswesens darstellt. ${ }^{378}$

\section{Zwischenfazit}

Ziel von Teil D der vorliegenden Arbeit war es, zur Beantwortung der ersten Forschungsfrage den Status quo der Integration des internen und externen Rechnungswesens bei den untersuchten Unternehmen der Hauptstichprobe zu ermitteln. Wie die Analyse gezeigt hat, weisen die Unternehmen ein insgesamt hohes Ausmaß an Integration auf, wobei die Integrationsintensität im Aktionsfeld 'Gestaltung der controllingrelevanten IT-Systeme' etwas schwächer und im Aktionsfeld 'Institutionalisierung der Controllerarbeit' deutlich stärker als in den drei originären Aktionsfeldern ausgeprägt ist. Die Betrachtung der anhand von unternehmensinternen bzw. umfeldbezogenen Einflussfaktoren gebildeten Integrationsprofile erlaubt darüber hinaus die Feststellung, dass jeweils diejenigen Unternehmen eine vergleichsweise stärkere Gesamtintegration des Rechnungswesens aufweisen, die

- börsennotiert sind,

- anhand ihrer Mitarbeiteranzahl von mehr als 10.000 als 'groß' einzustufen sind,

- als Leading GAAP die IFRS statt der HGB-Vorschriften anwenden oder

- einer geringen Volatilität ihres operativen Ergebnisses unterliegen.

Zudem ergaben sich auch innerhalb der einzeln betrachteten Aktionsfelder unterschiedlich starke Integrationsintensitäten im Zusammenhang mit den betrachteten Kontextfaktoren. So zeigt sich beispielsweise bei den in der Hauptstichprobe enthaltenen Tochterunternehmen ein im Vergleich zu den Holdings niedrigeres Integrationsni-

378 Vgl. hierzu International Group of Controlling (Hrsg.)/Weißenberger (2006), S. 25ff., Simons/ Weißenberger (2008), S. 138, Weißenberger (2008a), Rz. 59 sowie die Ausfuhrungen in Abschnitt D2.3 der vorliegenden Arbeit. 
veau innerhalb der Bereiche Planung und Performance-Messung. Auch weisen diejenigen Unternehmen, die in einem dynamischen Umfeld agieren, ein höheres Integrationsniveau im Bereich Institutionalisierung der Controllerarbeit auf.

Bei der abschließend durchgeführten hierarchiebezogenen Analyse konnten eine einheitlich starke sowie eine partielle Integration als die beiden dominierenden Integrationsmuster innerhalb der operativen Ergebnisplanung identifiziert werden. Auch hier deutet die kontextbezogene Analyse darauf hin, dass diejenigen Unternehmen, die kapitalmarktorientiert sind bzw. mit den IFRS ökonomisch fundierte und somit für die interne Steuerung vergleichsweise gut geeignete Rechnungslegungsstandards verwenden, verstärkt entweder das Muster der partiellen oder der einheitlich starken Integration des Rechnungswesens im Bereich der operativen Ergebnisplanung aufweisen. 


\section{E Einfluss der Integration des Rechnungswesens auf die Controllerarbeit}

Nachdem der Stand der Integration des internen und externen Rechnungswesens bei den Unternehmen der Hauptstichprobe umfassend beleuchtet wurde, ist nachfolgend im Rahmen der Beantwortung der Forschungsfragen 2 und 3 zu untersuchen, welchen Einfluss die Integrationsintensität auf die Controllerarbeit hat. Hierzu wird zunächst erfasst, in welchem Ausmaß die Controller die drei verschiedenen Rollen gemäß dem House of Controlling ausüben und welchen Einfluss die Integration des Rechnungswesens hierauf besitzt. Anschließend erfolgt die Analyse, inwieweit sich die Integrationsintensität auf den Erfolg der Controllerarbeit aus Sicht des Managements auswirkt.

\section{Rollenbezogene Ausgestaltung der Controllerarbeit im Kon- text der Integration des Rechnungswesens}

\subsection{Ausübung der Controllerrollen}

Der Finanzbereich deutscher Großunternehmen unterliegt in den letzten Jahren einem umfassenden Wandel, der auch unter dem Stichwort 'Finance Transformation' diskutiert wird. ${ }^{379}$ Neben der in dieser Arbeit im Mittelpunkt der Betrachtung stehenden zunehmenden Integration des Rechnungswesens sind in diesem Zusammenhang weitere relevante Entwicklungen anzuführen, wie z.B.

- die wachsende Kapitalmarktorientierung der Unternehmen und die damit einhergehende verpflichtende Finanzberichterstattung nach IFRS, ${ }^{380}$

- die Verschlankung bzw. Bündelung von Prozessen des Finanz- und Rechnungswesens in Shared Service Center als Folge gestiegener Effizienzanforderungen $^{381}$ sowie bestimmter Dienstleistungen an einen unternehmensexternen Anbieter wird auch von 'Business Process Outsourcing' bzw. - für den Fall, dass der Anbieter im Ausland angesiedelt ist - von 'Offshoring' gesprochen, vgl. Michel (2007), S. 289-292.
} 
- die durch eine stärkere Automatisierung und Standardisierung von Prozessabläufen ermöglichte Fokussierung des Controllings auf die Entscheidungsunterstützung des Managements im Sinne eines 'Business Partnering'. ${ }^{382}$

Aus den genannten Veränderungen ergeben sich zwangsläufig Konsequenzen für die Ausgestaltung der Controllerarbeit, worauf auch die in Theorie und Unternehmenspraxis jüngst vermehrt geführte Diskussion bezüglich der Rolle des Controllers hindeutet. ${ }^{383} \mathrm{Um}$ dem geschilderten Umbruch im Finanzbereich und den daraus resultierenden Auswirkungen auf die Controllerarbeit Rechnung zu tragen, ist zunächst im Rahmen der empirischen Analyse zu erfassen, wie die derzeitige Ausübung der drei Controllerrollen des House of Controlling bei den Unternehmen der Hauptstichprobe ausgestaltet ist. ${ }^{384}$ Hierzu wurden die Controller gebeten, den durchschnittlich von ihnen für jede der drei verschiedenen Rollen verwendeten zeitlichen Arbeitsaufwand prozentual anzugeben, sodass sich in Summe ein Gesamtzeitaufwand von $100 \%$ ergibt. Bei der Befragung wurde neben der bisher realisierten auch die von den Controllern angestrebte Zeitaufteilung berücksichtigt. Durch diese Vorgehensweise konnte neben dem Status quo zusätzlich erfasst werden, welche Rollenausübung die Controller grundsätzlich favorisieren bzw. zukünftig realisieren möchten.

Wie in Abbildung 21 ersichtlich ist, nehmen die Controller der Hauptstichprobe die Rolle als betriebswirtschaftlicher Berater des Managements aktuell am stärksten wahr (40,15\%), dicht gefolgt von der Rolle als Methoden- und Systemdienstleister mit einem relativen Zeitumfang von $38,23 \%$. Deutlich schwächer fällt hingegen die Ausübung der Rolle als Informationsdienstleister für die Bilanzierung mit einem durchschnittlichen Anteil von $21,62 \%$ aus. ${ }^{385}$ Zukünftig streben die Controller jedoch eine deutliche - und statistisch durchweg höchst signifikante ${ }^{386}$ - Änderung in der Gewichtung ihrer Rollenausübung an. So soll der Fokus vermehrt auf die Beraterrolle gelegt

Vgl. Michel (2006), S. 442 sowie Weißenberger (2007a), S. 44f.

Vgl. hierzu exemplarisch den Sammelband „Die neue Rolle des Controllers“ von Weber et al. (Hrsg.) (2008).

Zum House of Controlling vgl. die Ausführungen in Abschnitt B1.2 der vorliegenden Arbeit.

Immerhin wenden die befragten Controller derzeit somit rechnerisch ca. einen Arbeitstag pro Woche für die Unterstützung der externen Finanzberichterstattung auf.

Da jeder Controller sowohl hinsichtlich der bisherigen als auch der angestrebten Zeitaufteilung befragt wurde, handelt es sich bei den Antworten um zwei verbundene Stichproben. Daher erfolgt der Test auf Signifikanz der Mittelwertunterschiede anhand des Wilcoxon-Tests. Vgl. hierzu die Ausfuhrungen in Abschnitt C3.2 der vorliegenden Arbeit. 
werden (Ausweitung auf 53,10\%) und zwar zu Ungunsten sowohl der Methoden- und Systemdienstleisterrolle (Reduktion auf 28,81\%) als auch der Informationsdienstleisterrolle gegenüber der Bilanzierung (Reduktion auf 18,11\%).

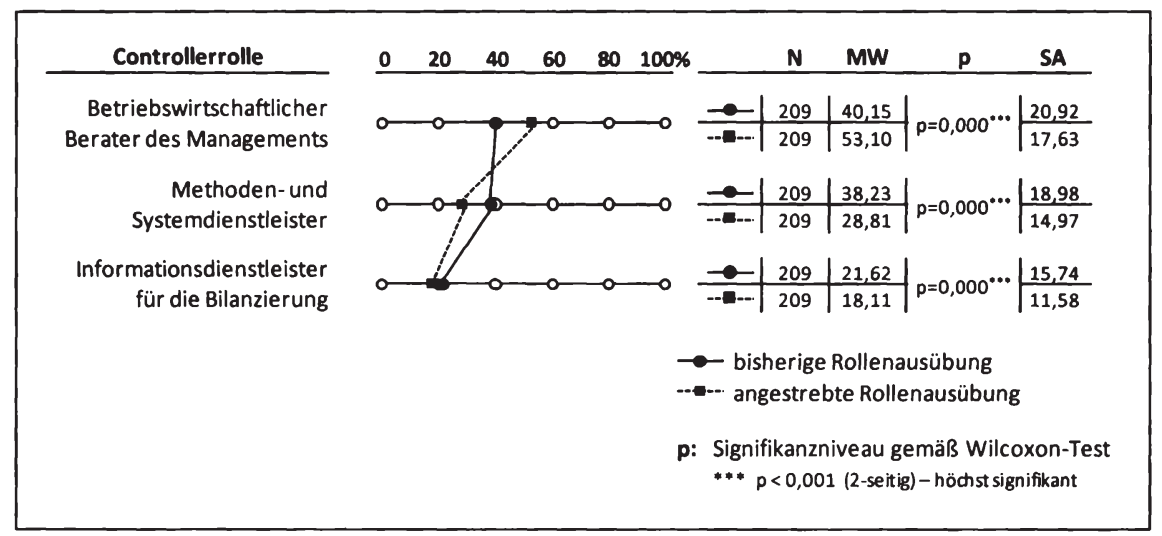

Abbildung 21: Bisherige und angestrebte Ausubung der Controllerrollen

Diese seitens der Controller angestrebte Rollenänderung reflektiert somit auch empirisch den Trend der Entwicklung des Controllers zum Business Partner. ${ }^{387}$ Der Schwerpunkt der Controllertätigkeit soll demnach nicht mehr wie zuvor in der Datengenerierung und -aufbereitung, sondern in der Beratung des Managements liegen. ${ }^{388}$

Die empirisch festgestellte Diskrepanz zwischen der derzeitigen Rollenausübung und der angestrebten Zeitaufteilung deutet darauf hin, dass diese Entwicklung offensichtlich noch nicht abgeschlossen ist. Ein ähnliches Indiz für diese Vermutung bilden die Ergebnisse der Studie von Weißenberger/Angelkort (2007b), die im Jahr 2006

$\mathrm{Zu}$ einer ähnlichen Aussage - wenn auch aus Managersicht - kommt eine Studie, die 2007 unter 25 in Deutschland borsennotierten Unternehmen durchgefuhrt wurde. Während $56 \%$ der befragten Chief Operating Officers zum Zeitpunkt der Datenerhebung die Controller ihres Unternehmens in der Rolle als 'Zahlenschmiede' sahen, gaben lediglich acht Prozent diese Controllerrolle als fur die Zukunft wünschenswert an. Die Rolle als Berater wurde dagegen von aktuell $48 \%$ der befragten Manager konstatiert und sogar von $80 \%$ für zukünftig erwünscht angesehen. Vgl. ausfuhrlich Ernst et al. (2008), hier S. 51. Daruber hinaus gaben in der von Weber et al. (2006) durchgefuhrten Studie $61,7 \%$ der 618 befragten Mitglieder des Internationalen Controller Vereins (ICV) an, dass das Management die Controller ihres Unternehmens in der Rolle eines internen Beraters sieht. Hingegen hielten sogar $73,2 \%$ der Befragten dieses Rollenbild für zukünftig erstrebenswert, vgl. Weber et al. (2006), S. 44f. 
dieselbe Fragestellung bezüglich der Rollengewichtung an österreichische, nach IFRS bilanzierende Unternehmen adressiert haben. ${ }^{389}$ Während sich die befragten Controller hier - im Gegensatz zu den empirischen Ergebnissen der vorliegenden Arbeit - zum Zeitpunkt der Befragung mit $45 \%$ noch überwiegend in der Rolle als Methoden- und Systemdienstleister involviert sahen, betrug der auf die Beraterrolle verwendete durchschnittliche Zeitumfang lediglich 38\%. Die Controller strebten jedoch ebenfalls eine deutliche Ausdehnung ihrer Beraterrolle auf 52\% an, wohingegen sie die Methodenund Systemdienstleisterrolle auf zukünftig 32\% ihrer Arbeitszeit reduzieren wollten. Die Reihenfolge der Bedeutung der beiden Controllerrollen war hier somit noch umgekehrt und der Unterschied zwischen der tatsächlichen und der angestrebten Ausübung dieser Rollen größer. ${ }^{390}$ Erwähnenswert ist zudem, dass die im Rahmen der Studie ebenfalls befragten Manager eine ähnliche Sichtweise hinsichtlich der Controllerrollenausübung zu erkennen gegeben haben. So befürworten sie eine Ausweitung der Rolle als betriebswirtschaftlicher Berater von derzeit 38\% auf zukünftig 48\%, während sie der Rolle als Methoden- und Systemdienstleister eine abnehmende Bedeutung (von $43 \%$ auf $32 \%$ ) attestieren. ${ }^{391}$ Offensichtlich unterstützen die Manager somit mehrheitlich den Trend der Entwicklung der Controller zum Business Partner.

\subsection{Einfluss der Integrationsintensität des Rechnungswesens auf die Ausübung der Controllerrollen}

Im zweiten Schritt der Beantwortung der zweiten Forschungsfrage soll der Einfluss der Integration des Rechnungswesens auf die Ausübung der Controllerrollen näher untersucht werden. Während die Bestandsaufnahme der Rollenausübung im vorangegangenen Abschnitt einer deskriptiv-explorativen Vorgehensweise entspricht, wird hierzu nunmehr ein explanativer, d.h. hypothesenbasierter, Ansatz gewählt.

In der Literatur wird angeführt, dass die Integration des Rechnungswesens eine stärkere Ausübung der Controllerrolle als betriebswirtschaftlicher Berater des Managements unterstützt, da aufgrund der Integration eine Vielzahl ansonsten doppelt

Vgl. zum Forschungsdesign dieser Studie die Ausführungen in Abschnitt B4.1 der vorliegenden Arbeit sowie ausführlich Weißenberger/Angelkort (2007b), im Folgenden insbesondere S. 34f. eingeschränkt zulässig ist, da die Datenerhebung in unterschiedlichen Ländern und aus unterschiedlich abgegrenzten Grundgesamtheiten erfolgt ist. 
vorhandener Prozesse, Systeme und Strukturen entfallen kann. ${ }^{392}$ So können etwa die für die interne Berichterstattung erforderlichen Ist-Daten weitestgehend direkt aus den Vorsystemen der Finanzbuchhaltung bzw. des externen Rechnungswesens übernommen werden. Das Ausmaß der notwendigen Abstimmungsarbeiten zwischen Controlling und Bilanzierung kann durch die Eliminierung bzw. Reduktion manueller Überleitungs- bzw. Nebenrechnungen sowie eine zentrale Datenspeicherung deutlich reduziert werden. Die hierdurch frei werdenden zeitlichen Kapazitäten kann der Controller für seine Rolle als Sparringspartner des Managements verwenden. Somit lassen sich die beiden folgenden Hypothesen aufstellen: ${ }^{393}$

$H_{1}$ : In Unternehmen mit einer im Vergleich zum Median höheren Gesamtintegration des Rechnungswesens üben Controller die Rolle als betriebswirtschaftlicher Berater des Managements in einem höheren Ausmaß aus als in Unternehmen, die eine geringere Integration des Rechnungswesens aufweisen.

$\mathrm{H}_{2}$ : In Unternehmen mit einer im Vergleich zum Median höheren Gesamtintegration des Rechnungswesens üben Controller die Rolle als Methodenund Systemdienstleister in einem geringeren Ausmaß aus als in Unternehmen, die eine geringere Integration des Rechnungswesens aufweisen.

Zur Überprüfung der Hypothesen wird in Abbildung 22 jeweils die derzeitige Ausübung der drei verschiedenen Controllerrollen in Abhängigkeit von der Ausprägung der Gesamtintegrationsintensität des Rechnungswesens dargestellt. Dabei bilden die Unternehmen, deren Gesamtintegrationsindex kleiner oder gleich dem Median ist, die erste Vergleichsgruppe, während jene Unternehmen, deren Gesamtintegrationsintensität höher als der Median liegt, in der zweiten Vergleichsgruppe berücksichtigt werden.

Vgl. auch im Folgenden Erichsen (2000), S. 58, Fleischer (2005), S. 195, Liedl (2005), S. 183, Michel (2006), S. 441 sowie Weißenberger (2007a), S. 44 und 218.

Bezüglich der Controllerrolle als Informationslieferant für die Bilanzierung wird an dieser Stelle keine Hypothese formuliert, da diese Rolle weniger mit der hier betrachteten Integration des Rechnungswesens, sondern mit dem Management Approach in Zusammenhang steht. Der Management Approach besagt, dass die intern seitens des Controllings für Zwecke der Unternehmenssteuerung zur Verfugung gestellten Informationen eine Zweitverwendung im Rahmen der externen Finanzberichterstattung finden. Vgl. hierzu ausfuhrlich Wagenhofer (2006), S. 4ff., Weißenberger/Maier (2006), Weißenberger (2007a), S. 169ff., Velte (2008) sowie Maier (2009). 


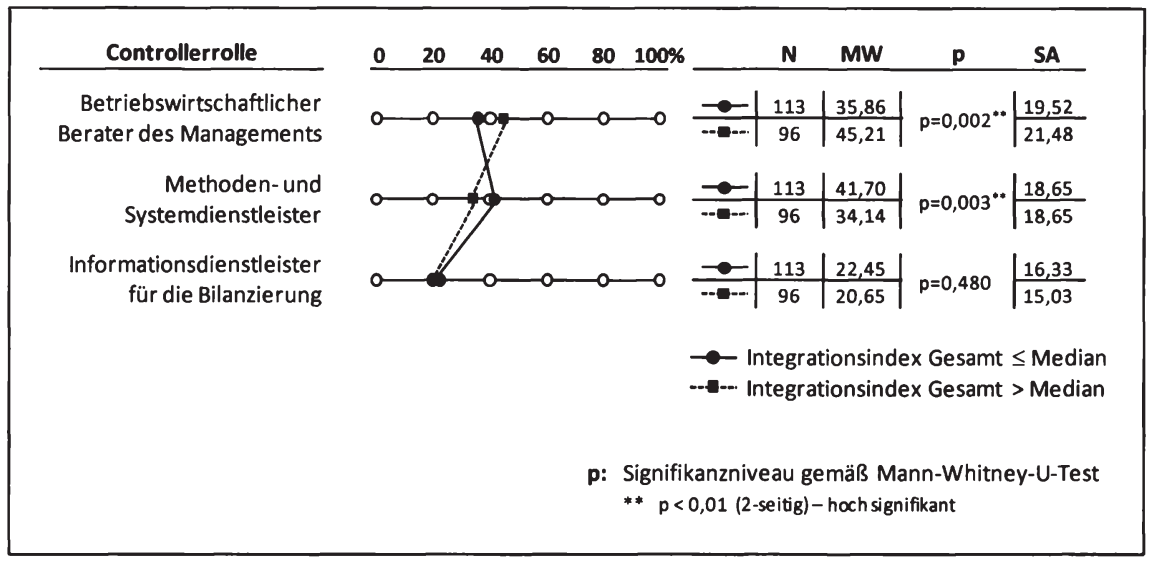

Abbildung 22: Ausübung der Controllerrollen in Abhăngigkeit der Gesamtintegrationsintensität des Rechnungswesens

Wie die empirische Analyse zeigt, nehmen die Controller der stärker integrierten Unternehmen die Rolle als betriebswirtschaftlicher Berater des Managements mit durchschnittlich $45,21 \%$ signifikant stärker wahr als es bei der schwächer integrierten Vergleichsgruppe der Fall ist (35,86\%). Hingegen entfällt bei den stärker integrierten Unternehmen mit 34,14\% ein signifikant geringerer Zeitanteil auf die Ausübung der Controllerrolle des Methoden- und Systemdienstleisters als bei den schwächer integrierten Unternehmen, deren Controller dieser Rolle mit $41,70 \%$ den größten Teil ihrer Arbeitszeit widmen. Die Ausprägung der Integrationsintensität des Rechnungswesens hat somit offenbar tatsächlich einen Einfluss auf die Ausübung der ersten beiden Controllerrollen. Die Hypothesen $\mathrm{H}_{1}$ und $\mathrm{H}_{2}$ gelten damit als bewährt.

Um diesen Zusammenhang noch genauer zu beleuchten und die diesbezüglichen Treiber zu identifizieren, erfolgt abschließend eine ergänzende Detailanalyse anhand der bereits in Abschnitt D2.2 vorgestellten aktionsfeldbezogenen Integrationssubindizes. Wie die in Abbildung 23 dargestellten Ergebnisse zeigen, ergibt sich innerhalb der originären Aktionsfelder der Controllerarbeit kein signifikanter Effekt der jeweiligen Integrationsintensität auf die Ausübung der Controllerrollen. Die Integration von internem und externem Rechnungswesen innerhalb der Planung, des Berichtwesens und der Performance-Messung ist für die Ausübung der Controllerrollen somit offenbar nicht bedeutsam. 


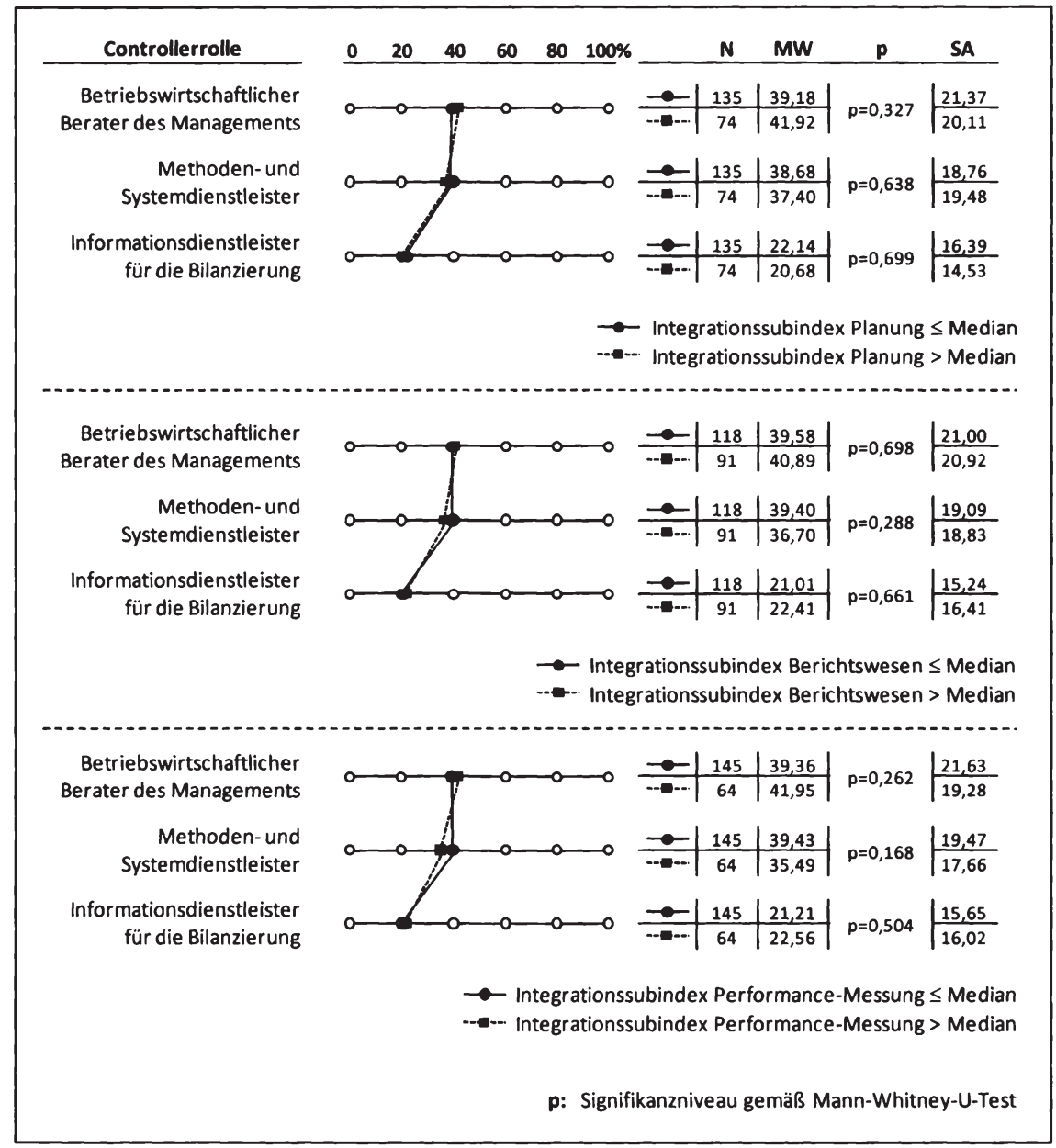

Abbildung 23: Ausübung der Controllerrollen in Abhăngigkeit der Integrationsintensităt des Rechnungswesens innerhalb der originären Aktionsfelder der Controllerarbeit

Dasselbe gilt für den Bereich der Institutionalisierung der Controllerarbeit innerhalb der derivativen Aktionsfelder, wie in Abbildung 24 ersichtlich ist. Die Gestaltung der controllingrelevanten IT-Systeme bildet hingegen den einzigen Bereich, dessen Integrationsintensität einen hoch bzw. höchst signifikanten Einfluss auf die Ausübung der ersten beiden Controllerrollen besitzt. So nehmen die Controller der stärker integrierten Unternehmen die Rolle als betriebswirtschaftlicher Berater des Managements 
mit durchschnittlich $46,59 \%$ deutlich stärker wahr als es bei der schwächer integrierten Vergleichsgruppe der Fall ist (34,14\%). Dagegen entfällt bei den stärker integrierten Unternehmen mit 33,54\% ein signifikant geringerer Zeitanteil auf die Ausübung der Controllerrolle des Methoden- und Systemdienstleisters als bei den schwächer integrierten Unternehmen, deren Controller auf diese Rolle mit 42,61\% den überwiegenden Teil ihrer Arbeitszeit verwenden.

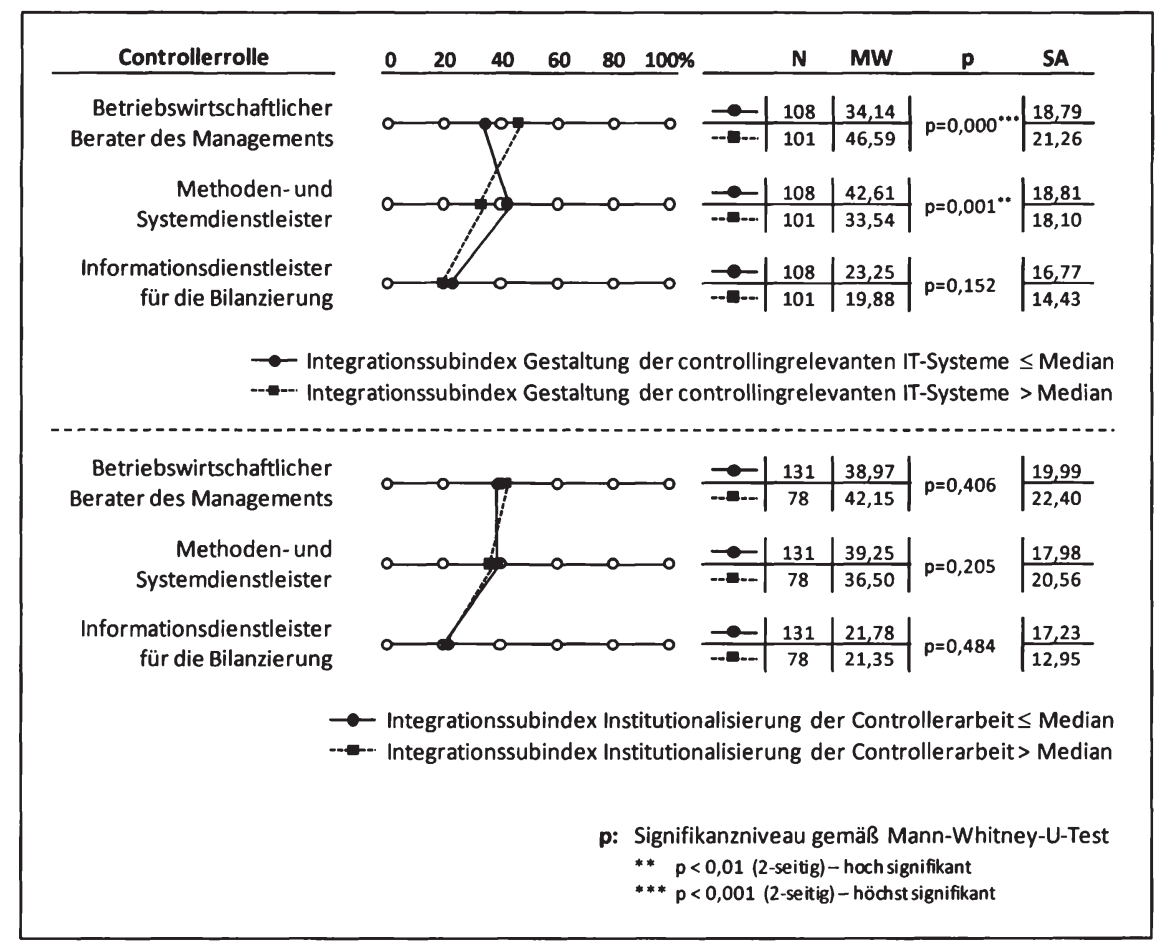

Abbildung 24: Ausübung der Controllerrollen in Abhăngigkeit der Integrationsintensităt des Rechnungswesens innerhalb der derivativen Aktionsfelder der Controllerarbeit

Die Ergebnisse der empirischen Detailanalyse deuten darauf hin, dass die strukturelle, inhaltliche, zeitliche sowie aufbau- bzw. ablauforganisatorische Integration des internen und externen Rechnungswesens innerhalb der Aktionsfelder Planung, Berichtswesen, Performance-Messung und Institutionalisierung der Controllerarbeit per se zunächst kein Freiwerden zeitlicher Ressourcen gewährleistet. Der zentrale Schlüssel zur Entwicklung des Controllers in Richtung Business Partner liegt hingegen in der 
Integration des Rechnungswesens innerhalb der controllingrelevanten IT-Systeme. Erst durch den Rückgriff auf eine integrierte und zentralisierte Systemlandschaft werden die Controller hinsichtlich ihrer Rolle als Methoden- und Systemdienstleister ausreichend entlastet, sodass eine verstärkte Konzentration auf die seitens der Controller selbst mehrheitlich angestrebte Beraterrolle ermöglicht wird.

$\mathrm{Zu}$ einem ähnlichen Ergebnis hinsichtlich der Bedeutung einer integrierten und zentralisierten Systemlandschaft kommen Scapens/Jazayeri (2003) im Rahmen einer Längsschnittuntersuchung, die in Form einer interviewbasierten Fallstudie in der Zeit zwischen 1993 und 1999 in einem europäischen Tochterunternehmen eines USamerikanischen Konzerns durchgeführt wurde. So begünstigte die in diesem Zeitraum erfolgte SAP-Einführung einen Rollenwandel der Management Accountants:

„Their jobs are completely different in SAP, than they were before. [...] No longer are the management accountants simply concerned with measuring and providing reports on business performance. [...] there is an important role for the management accountant in interpreting the various indicators of performance and showing how they relate to each other. [...] Such an integrative role, within the SAP environment, has potentially put management accountants [...] in the position of 'internal consultants' or analysts, who assist managers to create strategies and to take operating decisions. "'394

Zwar wurden die Ergebnisse dieser Untersuchung nicht explizit im Zusammenhang mit einer Integration des internen und externen Rechnungswesens gewonnen. Jedoch stellen die hier betrachteten Aspekte, d.h. der Einsatz eines ERP-Systems sowie die damit verbundene Datenintegration und -zentralisierung, ${ }^{395}$ ein wichtiges integrationskonstituierendes Merkmal im Aktionsfeld der Gestaltung der controllingrelevanten ITSysteme dar, da sie die systemtechnische Voraussetzung für eine einheitliche Datenbasis im Rahmen eines integrierten Rechnungswesens bilden. ${ }^{396}$ Zudem wird auch hier analog zur Interpretation der in Abbildung 24 dargestellten empirischen Ergebnisse der festgestellte Rollenwandel auf die aus der verstärkten IT-Unterstützung resultierende zeitliche Entlastung zurückgeführt: 
"The routinization/computerization of many traditional accounting tasks means that there is [...] space for management accountants to provide more direct support to business managers. "397

Zusammenfassend kann somit konstatiert werden, dass die Integration des internen und externen Rechnungswesens im Bereich der controllingrelevanten IT-Systeme den Rollenwandel der Controller in Richtung Business Partner bzw. betriebswirtschaftlicher Berater des Managements begünstigt.

\section{Einfluss der Integration des Rechnungswesens auf den Erfolg der Controllerarbeit}

Nachdem in den ersten beiden Forschungsfragen die Integrationsintensität des Rechnungswesens bei den Unternehmen der Hauptstichprobe detailliert erörtert und mit der Ausübung der drei Controllerrollen in Verbindung gesetzt worden ist, steht nun der Einfluss der Integrationsintensität des Rechnungswesens auf den Erfolg der Controllerarbeit - Letzterer beurteilt aus Sicht des Managements - im Vordergrund. Dabei wird der besonderen Bedeutung von Rechnungslegungsinformationen als Sprache bzw. Kommunikationsmedium Rechnung getragen. Es wird somit empirisch untersucht, inwieweit die Integration des Rechnungswesens mit der Fähigkeit der Controller in Verbindung steht, die übergeordnete Zielsetzung der Controllerarbeit des House of Controlling - diese liegt in der Übernahme von Mitverantwortung für die Erreichung der Unternehmensziele - zu erfüllen. Die Beantwortung dieser dritten Forschungsfrage wird mithilfe der Kovarianzstrukturanalyse auf Basis der dyadischen Stichprobe durchgeführt. ${ }^{398}$ Hierzu werden zunächst auf theoretisch-konzeptioneller Basis die zu Grunde liegenden Hypothesen abgeleitet sowie die damit verbundenen latenten Vari-

Scapens/Jazayeri (2003), S. 228.

Trotz der in Abschnitt C1.3.5 dargestellten hohen Leistungsfăhigkeit des SEM-Ansatzes wird diese Methodik im Rahmen der empirischen Controlling- bzw. Management AccountingForschung bislang vergleichsweise selten eingesetzt. So kommen Smith/Langfield-Smith (2004) im Zuge ihres für den Zeitraum zwischen 1980 und 2001 durchgefuhrten Reviews zu dem Ergebnis, dass in den zehn führenden (Management) Accounting Journals lediglich 20 Management Accounting-Beiträge auf SEM-Basis erschienen sind. Die Autoren konstatieren in diesem Zusammenhang (S. 79): „The greater use of SEM in MA [ = Management Accounting, d. Verf.] research [...] will enable the discipline to move beyond the restrictions imposed by more limited modeling techniques such as multiple regression and path analysis." 
ablen operationalisiert. ${ }^{399}$ Anschließend werden die Ergebnisse der Kovarianzstrukturanalyse erörtert.

\subsection{Hypothesenbildung}

Der in den Abschnitten A1 und B der vorliegenden Arbeit bereits ausfuhrlich dargestellten Integration des internen und externen Rechnungswesens wird sowohl aus konzeptioneller Sicht als auch seitens der Unternehmenspraxis eine positive Wirkung auf die Güte der Informationsversorgung durch das Controlling zugesprochen. So wird davon ausgegangen, dass die Controllinginformationen im Rahmen eines integrierten Rechnungswesens zum einen schneller bereitgestellt werden und zum anderen auch weniger fehleranfällig sind, da ein geringerer Überleitungsbedarf in Form von Abstimmbrücken notwendig ist. ${ }^{400}$ Hieraus resultieren präzisere Berichte, die sich durch eine erhöhte Aussagekraft auszeichnen. Daher lässt sich die folgende Hypothese formulieren, die auf die technische bzw. instrumentelle Ausgestaltung der Informationsversorgung des Managements durch das Controlling abzielt:

$H_{1}$ : Die Gesamtintegrationsintensität des Rechnungswesens wirkt positiv auf die Ergebnisqualität des Controllings.

Des Weiteren kann konstatiert werden, dass der Bedarf nach einer einheitlichen und transparenten Finanzsprache einen wichtigen Ausgangspunkt für die zunehmende Integration des internen und externen Rechnungswesens innerhalb der Unternehmenspraxis bildet. ${ }^{401}$ Diese Sichtweise betont eine konzeptionelle Nutzung ${ }^{402}$ von Rech-

Zur Abgrenzung der Begriffe 'Hypothese' bzw. 'Operationalisierung' vgl. Brühl (2008), S. 364ff. Vgl. auch im Folgenden Erichsen (2000), S. 58, Michel (2006), S. 441, Wagenhofer (2006), S. $12 \mathrm{f}$. sowie Weißenberger (2007c), S. $327 \mathrm{f}$.

Vgl. Fleischer (2005), S. 191, Moussallem (2006), S. 13, Weißenberger (2007c), S. 327 sowie Wenzel (2007), S. 301. Dieser Aspekt wird zudem dadurch empirisch untermauert, dass eine erleichterte Kommunikation als ein zentraler Vorteil einer Integration des Rechnungswesens angesehen wird. Vgl. hierzu die Ausfuhrungen zu den bisherigen empirischen Erkenntnissen hinsichtlich der Integration des Rechnungswesens in Abschnitt B4.2 der vorliegenden Arbeit.

Zur Klassifizierung unterschiedlicher Formen der Informationsnutzung - differenziert wird hier zwischen der instrumentellen, der konzeptionellen sowie der symbolischen Nutzung - vgl. grundlegend Menon/Varadarajan (1992), S. 54ff. Zur konzeptionellen Nutzung von Controllinginformationen vgl. ausführlich Heine (2008). 
nungslegungsinformationen als Sprache bzw. Kommunikationsmedium. ${ }^{403}$ Belkaoui (1989) stellt in diesem Zusammenhang fest:

"It is customary to call accounting a language or, more precisely, the language of business, since it is an important means of communicating information about a business concern. " 404

Die Verwendung von Rechnungslegungsinformationen beinhaltet - auch wenn diese aus vermeintlich 'harten' Zahlen bestehen - zwangsläufig eine interpretative Komponente, etwa im Zuge der Interpretation eines ermittelten Ergebnisses. ${ }^{405}$ Insbesondere im Falle einer Separation von internem und externem Rechnungswesen kann es hier zu Irritationen auf Seiten des Managements aufgrund der Inkonsistenz bzw. fehlenden Vergleichbarkeit der vorliegenden Finanzinformationen aus Controlling und Bilanzierung kommen. ${ }^{406}$ So gibt Belkaoui (1980) zu bedenken:

„Accountants from different professional groups may use different linguistic codes because of different organizational constraints and objectives. At worst, a confounding lack of communication may emerge. " 407

Die Integration des internen und externen Rechnungswesens fördert hingegen beim Management eine diesbezügliche Wahrnehmung im Sinne von 'one version of the truth', wodurch Konsistenz geschaffen und die besagten Interpretationsprobleme reduziert werden. ${ }^{408}$ Demnach lässt sich die folgende Hypothese ableiten:

$\mathrm{H}_{2}$ : Die Gesamtintegrationsintensität des Rechnungswesens wirkt positiv auf die Einheitlichkeit der Finanzsprache.

Vgl. hierzu grundlegend Belkaoui (1980), Otley (1980), S. 242, Boland/Pondy (1983), S. 228, Lavoie (1987), Belkaoui (1989), Pfaff/Weber (1998), Evans (2004) und Bloomfield (2008) sowie speziell im Kontext einer Integration des Rechnungswesens Schaier (2007), S. 188ff. und Simons/ Weißenberger (2009b), S. 450.

Belkaoui (1989), S. 282.

Vgl. Lavoie (1987), S. 599.

Ein anschauliches Zahlenbeispiel bezüglich der im Rahmen eines separierten Rechnungswesens auftretenden Divergenzen zwischen GuV-Jahresergebnis und Summe der internen Monatsergebnisse findet sich bei Simons/Weißenberger (2009a), S. 394f. Wagenhofer (2006) verdeutlicht anhand eines allgemein gehaltenen Beispiels die aus divergierenden Ergebnisgrößen resultierenden Kommunikationsprobleme innerhalb von Unternehmen sowie gegenüber Externen, vgl. Wagenhofer (2006), S. 13.

Belkaoui (1980), S. 362.

Vgl. Weißenberger (2007b), S. 15. 
Eine hohe Ausprägung der Einheitlichkeit der Finanzsprache fördert wiederum die Akzeptanz von Controllinginformationen seitens des Managements. ${ }^{409}$ Zur Begründung dieses verhaltenswissenschaftlich geprägten Zusammenhangs kann auf die von Festinger (1957) entwickelte Theorie der kognitiven Dissonanz zurückgegriffen werden. ${ }^{410}$ Diese besagt im Kern:

"1. The existence of dissonance, being psychologically uncomfortable, will motivate the person to try to reduce the dissonance and achieve consonance.

2. When dissonance is present, in addition to trying to reduce it, the person will actively avoid situations and information which would likely increase the dissonance. “411

Dabei gelten zwei Elemente als dissonant

„if, for one reason or another, they do not fit together. They may be inconsistent or contradictory [...]. "“412

Übertragen auf die Verwendung von Controllinginformationen bedeutet dies, dass es im Falle einer geringen Einheitlichkeit der Finanzsprache aufgrund der hieraus resultierenden Dissonanz zu einer tendenziell negativeren Wahrnehmung bzw. Beurteilung der bereitgestellten Controllinginformationen durch das Management kommt, welche ggf. zu einem Verzicht auf die Nutzung dieser Informationen im Rahmen der Entscheidungsfindung führt. ${ }^{413}$ Umgekehrt gesprochen würde die mit einer hohen Einheitlichkeit der Finanzsprache einhergehende Konsistenz der Finanzinformationen bewirken, dass Irritationen auf Seitens des Managements aufgrund von widersprüchlichen Steuerungsinformationen vermieden werden. Demzufolge fällt die Bewertung der Ergebnisqualität des Controllings durch das Management in diesem Fall entsprechend positiver aus, sodass die bereitgestellten Informationen vermehrt zur Entscheidungs-

409 Vgl. Weißenberger (2007c), S. 328.

410 Aufgrund ihrer einfachen Anwendbarkeit und hohen Plausibilität wird die Theorie der kognitiven Dissonanz innerhalb der betriebswirtschaftlichen Forschung insbesondere im Bereich des Marketings häufig eingesetzt. Vgl. Homburg/Stock (2001), S. 794.

411 Festinger (1957), S. 3.

412 Festinger (1957), S. 3f.

413 Sorter et al. (1964), S. 185, sprechen in einem ăhnlichen Zusammenhang von einer ,accounting intolerance of ambiguity [that] consists of assigning the same number to each financial event regardless of to whom, or for what purpose, the event is communicated." 
fundierung verwendet werden. ${ }^{414}$ Vor diesem Hintergrund lassen sich die beiden folgenden Hypothesen formulieren:

$H_{3}$ : Die Einheitlichkeit der Finanzsprache wirkt positiv auf die Ergebnisqualität des Controllings.

$H_{4}$ : Die Ergebnisqualität des Controllings wirkt positiv auf den Einfluss des Controllings auf Managemententscheidungen.

Das aus den Hypothesen $\mathrm{H}_{1}$ bis $\mathrm{H}_{4}$ resultierende Untersuchungsmodell ist in Abbildung 25 dargestellt. Es zeichnet sich durch einen dyadischen Charakter aus, da die Messung der exogenen Variablen auf den Antworten der befragten Controller basiert, während die Messung der endogenen Variablen die Sichtweise der korrespondierenden Manager widerspiegelt. Eine solche Verwendung unterschiedlicher Informationsquellen ermöglicht es, die Gefahr des Auftretens von Verzerrungen in der Kovarianzstruktur aufgrund des so genannten Common Method Bias zu reduzieren. ${ }^{415}$ Dieser Bias liegt vor, wenn die festgestellten Dependenzen zwischen unabhängigen und abhängigen Variablen nicht ausschließlich aus einem tatsächlichen Zusammenhang resultieren, sondern ebenfalls daraus, dass für die Messung beider Variablen auf dieselbe Informationsquelle zurückgegriffen wurde. ${ }^{416}$ Ein derartiges Phänomen kann insbesondere auf bewusste kognitive Vorgänge auf Seiten des Antwortenden zurückgeführt werden, wie etwa das Bestreben, sich in einer impliziten Theorien entsprechenden Weise hinsichtlich der Zusammenhänge zwischen den betrachteten Variablen zu äußern. ${ }^{417}$

Im Gegensatz zu einem Modell, welches ausschließlich auf Controllerantworten basiert, können im vorliegenden Fall folglich validere Erkenntnisse zur Auswirkung einer Integration des Rechnungswesens aus Nutzerperspektive - d.h. auf Basis der

414 Für eine empirische Bestătigung des letztgenannten Zusammenhangs auf Basis einzelner Items vgl. Angelkort/Sandt/Weißenberger (2008a), S. 191.

Vgl. Podsakoff et al. (2003), S. 887, Homburg/Klarmann (2006), S. 733 sowie Homburg (2007), S. 45.

416 Vgl. Podsakoff/Organ (1986), S. 533 sowie Homburg/Klarmann (2009), S. 149.

417 Vgl. Homburg/Klarmann (2009), S. 149. Podsakoff et al. (2003) unterscheiden insgesamt vier potenzielle Quellen - diese beinhalten auch weniger bewusste Prozesse - für das Auftreten eines Common Method Bias. Hierzu zählen 'common rater effects', 'item characteristic effects', 'item context effects' sowie 'measurement context effects'. Vgl. Podsakoff et al. (2003), S. 881ff. sowie ergänzend Söhnchen (2007), S. 138f. und Temme/Paulssen/Hildebrandt (2009), S. 124-126. 
Einschätzung der Manager bezüglich des Controllingerfolgs sowie der Einheitlichkeit der Finanzsprache - generiert werden.

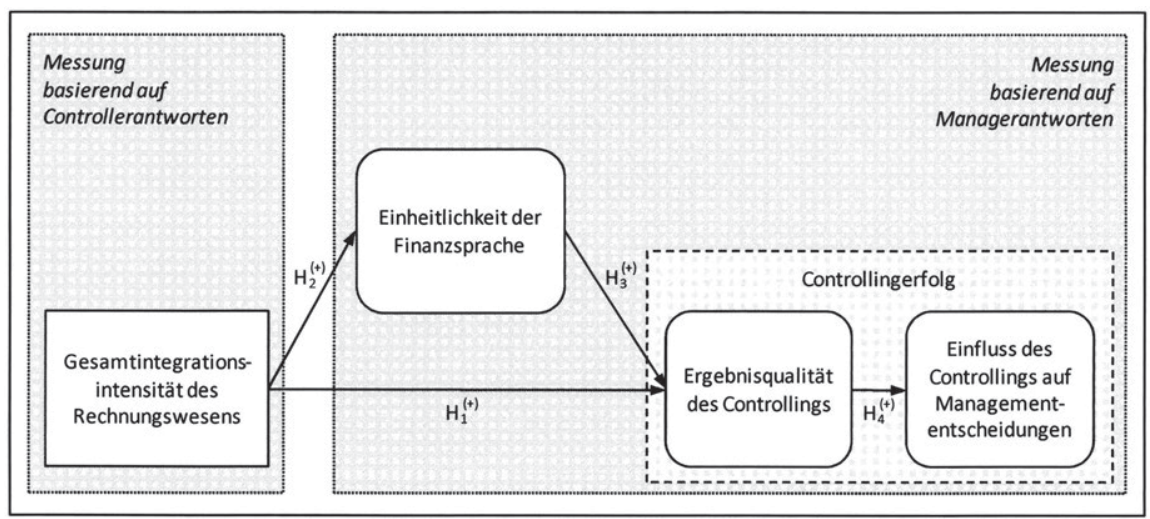

Abbildung 25: Dyadisches Untersuchungsmodell

Das der Analyse zu Grunde liegende Konzept von Controllingerfolg ist ebenfalls in Abbildung 25 ersichtlich. Neben der seitens des Managements wahrgenommenen Qualität des Outputs der Controllingabteilung ist diesbezüglich auch der Einfluss von Bedeutung, der dem Controlling im Rahmen der Entscheidungsfindung innerhalb des Unternehmens zuteil wird - dieser stellt das Outcome der Controllertätigkeit dar. Nur im Falle einer Kombination aus hoher Ergebnisqualität und hohem Einfluss ist die Übernahme von Mitverantwortung für die Erreichung der Unternehmensziele im Sinne einer erfolgreichen Controllerarbeit möglich.

\subsection{Operationalisierung der latenten Variablen}

\section{Gesamtintegrationsintensität des Rechnungswesens}

Die exogene Variable 'Gesamtintegrationsintensität des Rechnungswesens' bildet das Ausmaß ab, in dem das interne und das externe Rechnungswesen bei den untersuchten Unternehmen vereinheitlicht sind. Zur Messung der Variablen wird auf die im Rahmen der Ermittlung des Status quo der Integration in Abschnitt D1 vorgestellten 17 integrationsrelevanten Indikatoren zurückgegriffen, die sich über alle fünf Aktionsfelder der 
Controllerarbeit erstrecken. Einen deskriptiven Überblick über diese Items liefern die dortigen Tabellen 7 bis $11^{418}$

Anhand der zuvor in Tabelle 5 aufgelisteten Entscheidungsfragen wird deutlich, dass es sich bei der Gesamtintegrationsintensität des Rechnungswesens um eine formativ zu operationalisierende Variable handelt. So decken beispielsweise die Indikatoren nicht ein und dieselbe, sondern komplementäre Facetten der Integration des Rechnungswesens ab. Sie stellen somit definierende Charakteristika des Konstrukts dar, sodass die Kausalität vom jeweiligen Indikator zum Konstrukt verläuft. Folglich geht eine Änderung einer Indikatorvariablen auch nicht zwangsläufig mit einer Änderung der übrigen Indikatorvariablen einher.

Wie Diamantopoulos/Winklhofer (2001) zeigen, ist die Verwendung formativer Messmodelle im Rahmen der Kovarianzstrukturanalyse grundsätzlich möglich. ${ }^{419}$ Hierzu muss eine formative latente Variable identifiziert sein, d.h. es ist notwendig, dass von ihr mindestens zwei Pfade zu zwei reflektiven latenten Variablen ausgehen, die unkorreliert sind. ${ }^{420}$ Im Falle des oben beschriebenen Untersuchungsmodells ist diese Voraussetzung jedoch nicht erfültt, da die Variablen 'Einheitlichkeit der Finanzsprache' und 'Ergebnisqualität des Controllings' durch Hypothese $\mathrm{H}_{3}$ miteinander verbunden sind. Die Implementierung eines formativen Messmodells für die Variable 'Gesamtintegrationsintensität des Rechnungswesens' ist hier folglich nicht möglich.

Zur Lösung dieser Operationalisierungsproblematik wird für die Messung der exogenen Variablen auf den Gesamtintegrationsindex des Rechnungswesens zurückgegriffen. Dieser wurde in Abschnitt D2 als arithmetisches Mittel der 17 integrations-

Hierbei ist zu beachten, dass sich die dort angegebenen Informationen auf die Unternehmen der Hauptstichprobe beziehen. Im Rahmen der Beantwortung der dritten Forschungsfrage werden hiervon jedoch nur diejenigen Unternehmen berücksichtigt, von denen neben dem Controllerfragebogen auch der Managerfragebogen für die Analyse zur Verfügung stand. Da sich diese dyadische Stichprobe aber nicht signifikant von der Hauptstichprobe unterscheidet - dies wurde in Abschnitt C2.2 gezeigt - wird an dieser Stelle auf eine separate Übersichtsstatistik verzichtet.

Vgl. Diamantopoulos/Winklhofer (2001), S. 274. Die in der Literatur häufig aufzufindende Aussage, dass im Rahmen der kovarianzbasierten Strukturgleichungsanalyse lediglich reflektive Messmodelle spezifiziert werden können, stellt somit ein „weit verbreitetes Missverständnis“ (Scholderer/Balderjahn (2006), S. 65) dar.

Vgl. Bollen/Lennox (1991), S. 312 sowie MacCallum/Browne (1993), S. 539f. Ebenso können zwei Pfade zu zwei manifesten Variablen ausgehen. In diesem Fall liegt ein so genanntes

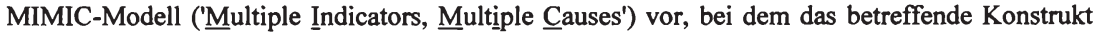
sowohl reflektiv als auch formativ gemessen wird. Vgl. Diamantopoulos/Winklhofer (2001), S. 272, Scholderer/Balderjahn (2006), S. 60 sowie grundlegend Hauser/Goldberger (1971). 
relevanten Indikatoren ermittelt. ${ }^{421}$ Wie Bollen/Lennox (1991) zeigen, kommt eine Indexbildung einer formativen Operationalisierung nahezu gleich. ${ }^{422}$ Eine solche Vorgehensweise kann daher als quasi-formative Operationalisierung angesehen bzw. bezeichnet werden. Der gebildete Index stellt jedoch keine latente Variable dar, sondern wird als manifeste Variable ins Untersuchungsmodell aufgenommen. ${ }^{423}$ Ersichtlich wird dies durch die rechteckige Darstellung der Variablen 'Gesamtintegrationsintensität des Rechnungswesens' in Abbildung 25. Einen deskriptiven Überblick über den zur Messung verwendeten Gesamtintegrationsindex (sowie die ebenfalls ermittelten aktionsfeldbezogenen Integrationssubindizes) gibt die zuvor bereits vorgestellte Abbildung 12.

\section{Ergebnisqualität des Controllings}

Die endogene latente Variable 'Ergebnisqualität des Controllings' misst die seitens des Managements wahrgenommene Qualität des Outputs der Controllingabteilung, beispielsweise hinsichtlich der Vollständigkeit, Genauigkeit und Aktualität der gelieferten Informationen. Sie stellt eine modifizierte Version eines von Bauer (2002) entwickelten Messmodells ${ }^{424}$ dar und besteht aus sechs reflektiven Indikatoren. Fünf (von acht) der ursprünglich auf einer siebenstufigen Rating-Skala gemessenen Items ${ }^{425}$ wurden von Bauer übernommen, während ein neuformulierter Indikator hinzugefügt wurde. Tabelle 17 enthält eine deskriptive Übersichtsstatistik bezüglich der dem Konstrukt zugehörigen Items.

421 Einen kovarianzstrukturanalytischen Vergleich unterschiedlicher Methoden zur Bildung von 'composite measures' - allerdings nicht unter dem Blickwinkel einer formativen Operationalisierung - unternehmen Landis/Beal/Tesluk (2000).

Die Verwendung eines Indexes entspricht demnach nahezu einem speziellen Fall eines formativen Messmodells, in dem alle Indikatoren gleich gewichtet sind und die Varianz der ResidualgröBe $\zeta$ null beträgt. Vgl. Bollen/Lennox (1991), S. 310. 


\begin{tabular}{|c|c|c|c|c|c|c|c|c|}
\hline \multirow{2}{*}{ Bezeichnung der Indikatoren } & \multirow{2}{*}{ MW } & \multirow{2}{*}{ SA } & \multicolumn{6}{|c|}{ Relative Antworthäufigkeit in \% } \\
\hline & & & 0 & 1 & 2 & 3 & 4 & 5 \\
\hline $\begin{array}{l}\text { Inhaltlich decken die Berichte alle wichtigen Bereiche } \\
\text { des Geschäfts ab. } \\
\qquad(0=\text { trifft gar nicht zu, ..., } 5=\text { trifft voll zu / N=149) }\end{array}$ & 3,94 & 1,04 & 0,67 & 4,03 & 3,36 & 15,44 & 45,64 & 30,87 \\
\hline $\begin{array}{l}\text { Das Controlling bildet mit seinem Informationssystem } \\
\text { die tatsächlichen Verhältnisse umfassend und wirklich- } \\
\text { keitsgetreu ab. } \\
\quad(0=\text { trifft gar nicht zu, ..., } 5=\text { trifft voll zu / N=149) }\end{array}$ & 3,90 & 0,84 & 0,00 & 2,68 & 2,68 & 16,78 & 57,72 & 20,13 \\
\hline $\begin{array}{l}\text { Die Informationen aus unserem Controlling sind sehr } \\
\text { genau. } \\
\qquad(0=\text { trifft gar nicht zu, ..., } 5=\text { trifft voll zu } / \mathrm{N}=149)\end{array}$ & 3,91 & 0,83 & 0,00 & 1,34 & 5,37 & 15,44 & 57,05 & 20,81 \\
\hline $\begin{array}{l}\text { Die Informationen aus unserem Controlling sind aktuell } \\
\text { genug. } \\
\qquad(0=\text { trifft gar nicht zu, ..., } 5=\text { trifft voll zu / } N=149)\end{array}$ & 3,77 & 1,06 & 0,67 & 4,70 & 4,70 & 20,81 & 45,64 & 23,49 \\
\hline $\begin{array}{l}\text { Unser Controlling verwendet nachvollziehbare Metho- } \\
\text { den und Techniken. } \\
\qquad(0=\text { trifft gar nicht zu, ..., } 5=\text { trifft voll zu / N=149) }\end{array}$ & 3,94 & 0,90 & 0,00 & 2,68 & 2,68 & 19,46 & 48,32 & 26,85 \\
\hline $\begin{array}{l}\text { Der Informationsgehalt bzw. die Aussagekraft der } \\
\text { Controllingberichte ist hoch. } \\
\qquad(0=\text { trifft gar nicht zu, ..., } 5=\text { trifft voll zu / } N=149)\end{array}$ & 3,78 & 0,95 & 0,67 & 2,68 & 4,03 & 23,49 & 48,99 & 20,13 \\
\hline
\end{tabular}

\section{Tabelle 17: Operationalisierung des Faktors 'Ergebnisqualität des Controllings'}

\section{Einheitlichkeit der Finanzsprache}

Bei der endogenen latenten Variablen 'Einheitlichkeit der Finanzsprache' handelt es sich um ein selbstentwickeltes Konstrukt. Es besteht aus drei reflektiven Indikatoren und misst, inwieweit die vom Controlling und von der Bilanzierung bereitgestellten Informationen zur wirtschaftlichen Situation des Unternehmens seitens des Managements als konsistent wahrgenommen werden. Einen deskriptiven Überblick über die einzelnen Items des Konstrukts gibt Tabelle 18.

\begin{tabular}{|c|c|c|c|c|c|c|c|c|}
\hline \multirow{2}{*}{ Bezeichnung der Indikatoren } & \multirow{2}{*}{ MW } & \multirow{2}{*}{ SA } & \multicolumn{6}{|c|}{ Relative Antworthäufigkeit in \% } \\
\hline & & & 0 & 1 & 2 & 3 & 4 & 5 \\
\hline $\begin{array}{l}\text { Controlling und Bilanzierung haben ein und dieselbe } \\
\text { Auffassung von Unternehmenserfolg. } \\
\qquad(0=\text { trifft gar nicht } \mathrm{zu}, \ldots, 5=\text { trifft voll zu / N=149) }\end{array}$ & 3,78 & 1,11 & 0,00 & 5,37 & 8,05 & 18,12 & 40,27 & 28,19 \\
\hline $\begin{array}{l}\text { Das Zahlenwerk des Controllings steht in einem einfach } \\
\text { nachvollziehbaren Zusammenhang mit dem Zahlenwerk } \\
\text { der Bilanzierung. } \\
\quad(0=\text { trifft gar nicht zu, .., } 5=\text { trifft voll zu / N=149) }\end{array}$ & 3,46 & 1,26 & 1,34 & 7,38 & 12,08 & 25,50 & 30,20 & 23,49 \\
\hline $\begin{array}{l}\text { Informationen aus Controlling und Bilanzierung ergeben } \\
\text { ein einheitliches Bild der Geschäftssituation. } \\
\qquad(0=\text { trifft gar nicht } \mathrm{zu}, \ldots, 5=\text { trifft voll } \mathrm{zu} / \mathrm{N}=149)\end{array}$ & 3,88 & 1,11 & 1,34 & 3,36 & 6,71 & 14,77 & 42,28 & 31,54 \\
\hline
\end{tabular}

Tabelle 18: Operationalisierung des Faktors 'Einheitlichkeit der Finanzsprache' 


\section{Einfluss des Controllings auf Managemententscheidungen}

Die endogene latente Variable 'Einfluss des Controllings auf Managemententscheidungen' spiegelt wider, wie der Einfluss des Controllings im Rahmen der Entscheidungsfindung innerhalb des Unternehmens vom Management beurteilt wird. Sie stellt somit das Outcome der Controllertätigkeit dar. Die Messung des Konstrukts erfolgt anhand eines von Spillecke (2006) entwickelten Instruments, welches drei reflektive Indikatorvariablen umfasst, die ursprünglich auf einer fünfstufigen Rating-Skala gemessen wurden. ${ }^{426}$ Eine deskriptive Übersichtsstatistik bezüglich der dem Konstrukt zugehörigen Items findet sich in Tabelle 19.

\begin{tabular}{|c|c|c|c|c|c|c|c|c|}
\hline \multirow{2}{*}{ Bezeichnung der Indikatoren } & \multirow{2}{*}{ MW } & \multirow{2}{*}{ SA } & \multicolumn{6}{|c|}{ Relative Antworthäufigkeit in \% } \\
\hline & & & 0 & 1 & 2 & 3 & 4 & 5 \\
\hline $\begin{array}{l}\text { Das Controlling spielt eine sehr wichtige Rolle bei der } \\
\text { Entscheidungsfindung in unserer Organisation. } \\
\qquad(0=\text { trifft gar nicht } z u, \ldots, 5=\text { trifft voll zu / N=149) }\end{array}$ & 3,84 & 0,98 & 1,34 & 0,00 & 7,38 & 21,48 & 44,30 & 25,50 \\
\hline $\begin{array}{l}\text { Das Management legt großen Wert auf die Meinung des } \\
\text { Controllings bei der Entscheidungsfindung. } \\
\quad(0=\text { trifft gar nicht } z \mathrm{u}, \ldots, 5=\text { trifft voll } \mathrm{zu} / \mathrm{N}=149)\end{array}$ & 3,81 & 0,91 & 0,67 & 1,34 & 4,03 & 25,50 & 47,65 & 20,81 \\
\hline $\begin{array}{l}\text { Das Controlling hat einen starken Einfluss auf die } \\
\text { Entscheidungen des Managements. } \\
\qquad(0=\text { trifft gar nicht } z u, \ldots, 5=\text { trifft voll zu / N=149) }\end{array}$ & 3,51 & 1,00 & 2,01 & 2,01 & 8,05 & 30,20 & 46,31 & 11,41 \\
\hline
\end{tabular}

Tabelle 19: Operationalisierung des Faktors 'Einfluss des Controllings auf Managemententscheidungen'

\subsection{Gütebeurteilung der Konstruktmessung}

Für die Berechnung der nachfolgend dargestellten kovarianzstrukturanalytischen Ergebnisse wurde auf die Software Amos, Version 17.0, zurückgegriffen. ${ }^{427}$ Entsprechend der Empfehlung von Homburg/Klarmann (2006) wurde hierbei die Maximum Likelihood (ML)-Methode als Schätzverfahren verwendet. ${ }^{428}$ Zwar stellt das Vorliegen

Vgl. Spillecke (2006), S. 161-164 sowie S. 239.

$\mathrm{Zu}$ Amos vgl. grundsätzlich Byrne (2001), Arbuckle (2007) sowie Blunch (2008). Einen Überblick über verfugbare Softwarepakete zur Berechnung von Strukturgleichungsmodellen gibt Reinecke (2005), S. $367 \mathrm{ff}$. Einen konkreten Anwendungsvergleich der Programme Lisrel und Amos (sowie SmartPLS fur eine varianzbasierte Vorgehensweise) unternimmt Jahn (2007). Ein anschauliches Fallbeispiel einer kovarianzstrukturanalytischen Untersuchung auf Basis von Amos findet sich bei Backhaus et al. (2006), S. 387ff. Da die hier zitierte Textstelle in der uberarbeiteten Druckversion der 12. Auflage nicht mehr enthalten ist, wird ausnahmsweise auf die 11. Auflage verwiesen.

Vgl. Homburg/Klarmann (2006), S. 736. Zum Maximum Likelihood-Schătzverfahren vgl. Schermelleh-Engel/Moosbrugger/Müller (2003), S. 25ff. sowie Reinecke (2005), S. 109f. 
einer multivariaten Normalverteilung der Indikatorvariablen grundsätzlich eine Anwendungsvoraussetzung für dieses Verfahren dar. ${ }^{429}$ In zahlreichen Simulationsstudien hat sich jedoch gezeigt, dass die ML-Methode robust gegenüber Abweichungen von der multivariaten Normalverteilungsannahme ist und es - wenn überhaupt - lediglich zu geringen Verzerrungen bei der Parameterschätzung kommt. ${ }^{430}$ Allerdings kann es $\mathrm{zu}$ einer Unterschätzung der Standardfehler kommen, die fehlerhafte Ergebnisse bezüglich der statistischen Signifikanz der standardisierten Effekte nach sich zieht. ${ }^{431}$ Daher wird in der vorliegenden Untersuchung zusätzlich das Bootstrapping-Verfahren verwendet, da dieses eine verlässliche Signifikanzprüfung direkter und insbesondere indirekter Effekte erlaubt. ${ }^{432}$ So gilt diese Methode als

„particularly useful [...] when distribution assumptions have been violated “433

und wird dementsprechend in jüngster Zeit vermehrt eingesetzt. ${ }^{434}$

Hinsichtlich der Gütebeurteilung von formativen Konstrukten stellen Diamantopoulos/Winklhofer (2001) fest, dass

„conventional procedures used to assess the validity and reliability of scales composed of reflective indicators (e.g., factor analysis and assessment of internal consistency) are not appropriate for composite variables (i.e., indexes) with formative indicators. “435

Im selben Zusammenhang weisen Scholderer/Balderjahn (2006) darauf hin, dass sich aufgrund des definitorischen Charakters eines formativen Messmodells und der damit einhergehenden Unzulässigkeit alternativer Operationalisierungen die Frage der Konstruktvalidität ohnehin nicht stellt. ${ }^{436}$ Für die exogene Variable 'Gesamtintegrations-

Vgl. Byrne (2001), S. 70.

Vgl. Homburg/Klarmann (2006), S. 736 sowie die dort angegebenen Studien.

Vgl. Byrne (2001), S. 268.

Vgl. hierzu die Ausführungen in Abschnitt C3.5 der vorliegenden Arbeit.

Cheung/Lau (2008), S. 301. Eine ähnliche Feststellung findet sich bei Shrout/Bolger (2002), S. 440: "The use of the bootstrap to estimate and to test direct and indirect effects can help with mediation problems in which the mediator and outcome variables are not normally distributed."

Vgl. Cheung/Lau (2008), S. 317.

Diamantopoulos/Winklhofer (2001), S. 271.

Vgl. Scholderer/Balderjahn (2006), S. 65. In ähnlicher Weise merkt Bagozzi (1994), S. 333 an, dass ,reliability in the internal consistency sense and construct validity in terms of convergent and discriminant validity are not meaningful when indexes are formed as a linear sum of measurements." 
intensität des Rechnungswesens' ist somit aufgrund deren quasi-formativen Operationalisierung eine formale Gütebeurteilung nicht sinnvoll anwendbar.

Dagegen ist grundsätzlich die Vollständigkeit formativer Indikatoren von entscheidender Bedeutung, damit sichergestellt wird, dass sämtliche Ursachen eines Phänomens berücksichtigt werden. ${ }^{437} \mathrm{Da}$ hierfür allerdings kein formales Überprüfungsverfahren existiert, erwartet Homburg (2007):

„Gerade um den Mangel formaler Überprüfungsmöglichkeiten zu kompensieren, wird es in Zukunft jedoch immer wichtiger werden, über eine theoretischkonzeptionelle Argumentation und intensive Pretests die inhaltliche Validität formativer Messmodelle sicherzustellen und zu dokumentieren. “438

Dieser Anforderung wurde bei der Indexkonstruktion, auf die für die quasi-formative Operationalisierung der exogenen Variablen zurückgegriffen wurde, Rechnung getragen. So wurden zum einen die 17 integrationsrelevanten Einzelindikatoren entlang der Aktionsfelder des House of Controlling und somit anhand eines konzeptionellen Bezugsrahmens entwickelt. ${ }^{49}$ Zum anderen wurden sämtliche Fragestellungen im Vorfeld der Datenerhebung einem ausführlichen Pretest unterzogen und gegebenenfalls optimiert bzw. ergänzt. ${ }^{440}$ Folglich ist davon auszugehen, dass alle zentralen Facetten der Integration des Rechnungswesens erfasst wurden und demnach Inhaltsvalidität gewährleistet ist.

Eine formale Beurteilung der Operationalisierungsgüte erfolgt aus den oben genannten Gründen lediglich für die auf reflektiven Indikatoren basierenden endogenen latenten Variablen. In Abbildung 26 ist das - der Gleichung (2) aus Abschnitt C3.5 folgende - Messmodell der Untersuchung hinsichtlich der drei endogenen latenten Variablen in Matrixnotation dargestellt. ${ }^{441}$

437 Vgl. auch im Folgenden Homburg (2007), S. 41.

438 Homburg (2007), S. 41.

439 Zum House of Controlling vgl. Abschnitt B1.2, zur Indexkonstruktion Abschnitt D2.1 der vorliegenden Arbeit.

440 Zur Durchfuhrung des Pretests vgl. Abschnitt C1.2 der vorliegenden Arbeit.

441 Auf Seiten der exogenen Variablen 'Gesamtintegrationsintensität des Rechnungswesens' gilt, da diese als manifeste Variable in das Untersuchungsmodell aufgenommen wird und keinen Fehlerterm besitzt: $\mathbf{x}_{1}=\xi_{1}$. 
$\left(\begin{array}{l}y_{1} \\ y_{2} \\ y_{3} \\ y_{4} \\ y_{5} \\ y_{6} \\ y_{7} \\ y_{8} \\ y_{9} \\ y_{10} \\ y_{11} \\ y_{12}\end{array}\right)=\left(\begin{array}{lll}\lambda_{11} & 0 & 0 \\ \lambda_{21} & 0 & 0 \\ \lambda_{31} & 0 & 0 \\ 0 & \lambda_{42} & 0 \\ 0 & \lambda_{52} & 0 \\ 0 & \lambda_{62} & 0 \\ 0 & \lambda_{72} & 0 \\ 0 & \lambda_{82} & 0 \\ 0 & \lambda_{92} & 0 \\ 0 & 0 & \lambda_{10,3} \\ 0 & 0 & \lambda_{11,3} \\ 0 & 0 & \lambda_{12,3}\end{array}\right) *\left(\begin{array}{l}\eta_{1} \\ \eta_{2} \\ \eta_{3}\end{array}\right)+\left(\begin{array}{l}\varepsilon_{1} \\ \varepsilon_{2} \\ \varepsilon_{3} \\ \varepsilon_{4} \\ \varepsilon_{5} \\ \varepsilon_{6} \\ \varepsilon_{7} \\ \varepsilon_{8} \\ \varepsilon_{9} \\ \varepsilon_{10} \\ \varepsilon_{11} \\ \varepsilon_{12}\end{array}\right)$

Y: Indikatoren der jeweils zugehörigen endogenen latenten Variablen $\eta_{1}, \eta_{2}$ oder $\eta_{3}$

$\lambda$ : Faktorladungen der Indikatoren auf die jeweils zugehörige endogene latente Variable $\eta_{1}, \eta_{2}$ oder $\eta_{3}$

$\eta_{1}$ : Endogene latente Variable 'Einheitlichkeit der Finanzsprache'

$\eta_{2}$ : Endogene latente Variable 'Ergebnisqualität des Controllings'

$\eta_{3}$ : Endogene latente Variable 'Einfluss des Controllings auf Managemententscheidungen'

$\varepsilon:$ Messfehler der Indikatoren

Abbildung 26: Messmodell der Untersuchung hinsichtlich der endogenen latenten Variablen in Matrixnotation

Wie in der nachfolgenden Tabelle 20 ersichtlich ist, erfüllt die Operationalisierung des Faktors 'Ergebnisqualität des Controllings' die zuvor in Tabelle 6 vorgestellten Gütekriterien ausnahmslos und kann somit als ausgezeichnet bewertet werden. 


\begin{tabular}{|c|c|c|c|c|}
\hline \multicolumn{5}{|c|}{ Informationen zu den einzelnen Indikatoren des Faktors 'Ergebnisqualität des Controllings' } \\
\hline \multicolumn{2}{|l|}{ Bezeichnung der Indikatoren } & $\begin{array}{l}\text { Item to Total- } \\
\text { Korrelation }\end{array}$ & $\begin{array}{l}\text { Indikator- } \\
\text { reliabilität }\end{array}$ & $\begin{array}{l}t \text {-Wert der } \\
\text { Faktorladung }\end{array}$ \\
\hline \multicolumn{2}{|c|}{$\begin{array}{l}\text { Inhaltlich decken die Berichte alle wichtigen Bereiche } \\
\text { des Geschäfts ab. }\end{array}$} & 0,69 & 0,54 & 10,10 \\
\hline \multicolumn{2}{|c|}{$\begin{array}{l}\text { Das Controlling bildet mit seinem Informationssystem } \\
\text { die tatsächlichen Verhältnisse umfassend und wirklich- } \\
\text { keitsgetreu ab. }\end{array}$} & 0,81 & 0,75 & 12,95 \\
\hline \multicolumn{2}{|c|}{$\begin{array}{l}\text { Die Informationen aus unserem Controlling sind sehr } \\
\text { genau. }\end{array}$} & 0,75 & 0,65 & 11,50 \\
\hline \multicolumn{2}{|c|}{$\begin{array}{l}\text { Die Informationen aus unserem Controlling sind aktuell } \\
\text { genug. }\end{array}$} & 0,74 & 0,60 & 10,88 \\
\hline \multicolumn{2}{|c|}{$\begin{array}{l}\text { Unser Controlling verwendet nachvollziehbare Metho- } \\
\text { den und Techniken. }\end{array}$} & 0,63 & 0,45 & 8,91 \\
\hline \multicolumn{2}{|c|}{$\begin{array}{l}\text { Der Informationsgehalt bzw. die Aussagekraft der } \\
\text { Controllingberichte ist hoch. }\end{array}$} & 0,78 & 0,68 & 11,98 \\
\hline \multicolumn{5}{|c|}{ Informationen zum Faktor 'Ergebnisqualität des Controllings' } \\
\hline \multicolumn{2}{|l|}{ Deskriptives Gütekriterium } & \multicolumn{3}{|c|}{ Ergebnis der explorativen Faktorenanalyse } \\
\hline Cronbach'sches Alpha & 0,90 & Erklärte Varia & & 0,62 \\
\hline \multicolumn{5}{|c|}{ Ergebnisse der konfirmatorischen Faktorenanalyse } \\
\hline$\chi^{2}$-Wert & 7,44 & RMSEA & & 0,00 \\
\hline df (Freiheitsgrade) & 9 & SRMR & & 0,02 \\
\hline$\chi^{2} / \mathrm{df}$ & 0,83 & GFI & & 0,99 \\
\hline p-Wert & 0,59 & AGFI & & 0,97 \\
\hline Faktorreliabilität & 0,90 & $\mathrm{CFI}$ & & 1,00 \\
\hline Durchschnittlich erfasste Varianz (DEV) & 0,61 & TLI & & 1,00 \\
\hline
\end{tabular}

Tabelle 20: Informationen zum Faktor 'Ergebnisqualităt des Controllings'

Auch die Messung des Faktors 'Einheitlichkeit der Finanzsprache' hält die geforderten Schwellenwerte - soweit anwendbar - weitestgehend ein, wie Tabelle 21 zeigt. Die einzige Ausnahme bildet hierbei die erste Indikatorvariable, deren Indikatorreliabilität mit 0,38 nur leicht unterhalb des geforderten Wertes von 0,4 liegt. In Anbetracht der Tatsache, dass die Faktorladung dieses Items jedoch statistisch signifikant ist und es sich bei der Einheitlichkeit der Finanzsprache um ein vollständig neuentwickeltes Konstrukt handelt, erscheint diese marginale Abweichung akzeptabel und eine Eliminierung des Indikators nicht notwendig. ${ }^{442}$ 


\begin{tabular}{|c|c|c|c|c|}
\hline \multicolumn{5}{|c|}{ Informationen zu den einzelnen Indikatoren des Faktors 'Einheitlichkeit der Finanzsprache' } \\
\hline \multicolumn{2}{|l|}{ Bezeichnung der Indikatoren } & $\begin{array}{l}\text { Item to Total- } \\
\text { Korrelation }\end{array}$ & $\begin{array}{l}\text { Indikator- } \\
\text { reliabilität }\end{array}$ & \begin{tabular}{|c|}
-Wert der \\
Faktorladung
\end{tabular} \\
\hline \multicolumn{2}{|c|}{$\begin{array}{l}\text { Controlling und Bilanzierung haben ein und dieselbe } \\
\text { Auffassung von Unternehmenserfolg. }\end{array}$} & 0,57 & 0,38 & 7,81 \\
\hline \multicolumn{2}{|c|}{$\begin{array}{l}\text { Das Zahlenwerk des Controllings steht in einem einfach } \\
\text { nachvollziehbaren Zusammenhang mit dem Zahlenwerk } \\
\text { der Bilanzierung. }\end{array}$} & 0,71 & 0,69 & 10,84 \\
\hline \multicolumn{2}{|c|}{$\begin{array}{l}\text { Informationen aus Controlling und Bilanzierung ergeben } \\
\text { ein einheitliches Bild der Geschäftssituation. }\end{array}$} & 0,74 & 0,78 & 11,58 \\
\hline \multicolumn{5}{|c|}{ Informationen zum Faktor 'Einheitlichkeit der Finanzsprache' } \\
\hline \multicolumn{2}{|l|}{ Deskriptives Gütekriterium } & \multicolumn{3}{|c|}{ Ergebnis der explorativen Faktorenanalyse } \\
\hline Cronbach'sches Alpha & 0,82 & Erklärte Vari & & 0,62 \\
\hline \multicolumn{5}{|c|}{ Ergebnisse der konfirmatorischen Faktorenanalyse } \\
\hline$\chi^{2}$-Wert & $-*$ & \multicolumn{2}{|l|}{ RMSEA } & $-*$ \\
\hline df (Freiheitsgrade) & 0 & \multicolumn{2}{|l|}{ SRMR } & $-*$ \\
\hline$\chi^{2} / d f$ & $-*$ & \multicolumn{2}{|l|}{ GFI } & $-*$ \\
\hline p-Wert & $-*$ & \multicolumn{2}{|l|}{ AGFI } & $-*$ \\
\hline Faktorreliabilität & 0,83 & \multicolumn{2}{|l|}{$\mathrm{CFI}$} & $-*$ \\
\hline Durchschnittlich erfasste Varianz (DEV) & 0,62 & \multicolumn{2}{|l|}{ TLI } & -* \\
\hline
\end{tabular}

Tabelle 21: Informationen zum Faktor 'Einheitlichkeit der Finanzsprache'

Wie die nachfolgende Tabelle 22 erkennen lässt, kann auch für den Faktor 'Einfluss des Controllings auf Managemententscheidungen' eine reliable und valide Messung konstatiert werden, da die anwendbaren Gütekriterien ausnahmslos erfüllt sind.

Neben der Beurteilung der Reliabilität und Validität bzw. Anpassungsgüte jedes einzelnen Messmodells ist des Weiteren relevant, ob sich die im Untersuchungsmodell enthaltenen latenten Variablen in ausreichendem Maße voneinander unterscheiden und somit Diskriminanzvalidität gegeben ist. ${ }^{443}$ Zur Überprüfung der Diskriminanzvalidität wird auf das bereits in Abschnitt C3.4 der vorliegenden Arbeit vorgestellte Fornell/Larcker-Kriterium zurückgegriffen. Wie die Ergebnisse in Tabelle 23 zeigen, sind die jeweils durchschnittlich erfassten Varianzen (DEV) für jede mögliche Variablenkombination höher als die quadrierte Korrelation beider Variablen. Folglich ist das Fornel//Larcker-Kriterium erfüllt, und es kann von Diskriminanzvalidität der latenten Variablen ausgegangen werden. Somit kann zur Analyse der Anpassungsgüte des Gesamtmodells sowie zur Überprüfung der Strukturhypothesen übergegangen werden. 


\begin{tabular}{|c|c|c|c|c|}
\hline \multicolumn{5}{|c|}{$\begin{array}{l}\text { Informationen zu den einzelnen Indikatoren des Faktors 'Einfluss des Controllings auf Management- } \\
\text { entscheidungen' }\end{array}$} \\
\hline \multicolumn{2}{|l|}{ Bezeichnung der Indikatoren } & $\begin{array}{c}\text { Item to Total- } \\
\text { Korrelation }\end{array}$ & $\begin{array}{l}\text { Indikator- } \\
\text { reliabilität }\end{array}$ & $\begin{array}{l}t \text {-Wert der } \\
\text { Faktorladung }\end{array}$ \\
\hline \multicolumn{2}{|c|}{$\begin{array}{l}\text { Das Controlling spielt eine sehr wichtige Rolle bei der } \\
\text { Entscheidungsfindung in unserer Organisation. }\end{array}$} & 0,80 & 0,76 & 12,72 \\
\hline \multicolumn{2}{|c|}{$\begin{array}{l}\text { Das Management legt großen Wert auf die Meinung des } \\
\text { Controllings bei der Entscheidungsfindung. }\end{array}$} & 0,80 & 0,77 & 12,81 \\
\hline \multicolumn{2}{|c|}{$\begin{array}{l}\text { Das Controlling hat einen starken Einfluss auf die } \\
\text { Entscheidungen des Managements. }\end{array}$} & 0,76 & 0,67 & 11,69 \\
\hline \multicolumn{5}{|c|}{ Informationen zum Faktor 'Einfluss des Controllings auf Managemententscheidungen' } \\
\hline \multicolumn{2}{|l|}{ Deskriptives Gütekriterium } & \multicolumn{3}{|c|}{ Ergebnis der explorativen Faktorenanalyse } \\
\hline Cronbach'sches Alpha & 0,89 & Erklärte Varia & & 0,74 \\
\hline \multicolumn{5}{|c|}{ Ergebnisse der konfirmatorischen Faktorenanalyse } \\
\hline$\chi^{2}$-Wert & $-*$ & RMSEA & & $-*$ \\
\hline df (Freiheitsgrade) & 0 & SRMR & & $-*$ \\
\hline$\chi^{2} / d f$ & $-*$ & GFI & & $-*$ \\
\hline p-Wert & $-*$ & AGFI & & $-*$ \\
\hline Faktorreliabilität & 0,89 & $\mathrm{CFI}$ & & $-*$ \\
\hline Durchschnittlich erfasste Varianz (DEV) & 0,73 & TLI & & $-*$ \\
\hline
\end{tabular}

* Ein konfirmatorisches Faktorenmodell mit drei Indikatoren hat keine Freiheitsgrade. Die Berechnung der fehlenden Maße ist daher nicht sinnvoll.

Tabelle 22: Informationen zum Faktor 'Einfluss des Controllings auf Managemententscheidungen'

\begin{tabular}{|c|c|c|c|c|}
\hline \multicolumn{5}{|c|}{ Diskriminanzvalidität: Fornell/Larcker-Kriterium (DEV > quadrierte Korrelation) } \\
\hline Latente Variable & & $\begin{array}{l}\text { Einheitlichkeit der } \\
\text { Finanzsprache }\end{array}$ & $\begin{array}{l}\text { Ergebnisqualität } \\
\text { des Controllings }\end{array}$ & $\begin{array}{c}\text { Einfluss des } \\
\text { Controllings } \\
\text { auf Management- } \\
\text { entscheidungen }\end{array}$ \\
\hline & DEV & 0,62 & 0,61 & 0,73 \\
\hline $\begin{array}{l}\text { Einheitlichkeit der } \\
\text { Finanzsprache }\end{array}$ & 0,62 & \multirow{3}{*}{\multicolumn{3}{|c|}{ quadrierte Korrelationen }} \\
\hline $\begin{array}{l}\text { Ergebnisqualität des } \\
\text { Controllings }\end{array}$ & 0,61 & & & \\
\hline $\begin{array}{l}\text { Einfluss des Controllings } \\
\text { auf Management- } \\
\text { entscheidungen }\end{array}$ & 0,73 & & & \\
\hline
\end{tabular}

Tabelle 23: Ergebnisse der Ũberprüfung der Diskriminanzvalidităt anhand des Fornell Larcker-Kriteriums 


\section{4 Überprüfung der Hypothesen des Kovarianzstrukturmodells}

Abbildung 27 zeigt das aus den Hypothesen $\mathrm{H}_{1}$ bis $\mathrm{H}_{4}$ resultierende Strukturmodell in Matrixnotation. ${ }^{444}$

$$
\left(\begin{array}{l}
\eta_{1} \\
\eta_{2} \\
\eta_{3}
\end{array}\right)=\left(\begin{array}{ccc}
0 & 0 & 0 \\
\beta_{21} & 0 & 0 \\
0 & \beta_{32} & 0
\end{array}\right) *\left(\begin{array}{l}
\eta_{1} \\
\eta_{2} \\
\eta_{3}
\end{array}\right)+\left(\begin{array}{c}
\gamma_{11} \\
\gamma_{21} \\
0
\end{array}\right) *\left(\xi_{1}\right)+\left(\begin{array}{l}
\zeta_{1} \\
\zeta_{2} \\
\zeta_{3}
\end{array}\right)
$$

$\eta_{1}$ : Endogene latente Variable 'Einheitlichkeit der Finanzsprache'

$\eta_{2}$ : Endogene latente Variable 'Ergebnisqualität des Controllings'

$\eta_{3}$ : Endogene latente Variable 'Einfluss des Controllings auf Managemententscheidungen'

$\beta$ : Strukturkoeffizienten zwischen den endogenen latenten Variablen $\eta_{1}, \eta_{2}, \eta_{3}$

$\gamma$ : Strukturkoeffizienten zwischen der exogenen Variablen $\xi_{1}$ und den endogenen latenten Variablen $\eta_{1}, \eta_{2}, \eta_{3}$

$\xi_{1}$ : Exogene Variable 'Gesamtintegrationsintensität des Rechnungswesens'

$\zeta$ : Residualgröße der jeweils zugehörigen endogenen latenten Variablen $\eta_{1}, \eta_{2}$ oder $\eta_{3}$

\section{Abbildung 27: Strukturmodell in Matrixnotation}

Die Modellschätzung weist - bei einem p-Wert von 0,58 - eine hohe Anpassungsgüte auf, wie die ausnahmslose Erfüllung der globalen Gütekriterien der zweiten

\begin{tabular}{|l|c|c|}
\hline Gütekriterien der zweiten Generation & Anspruchsniveau & Ergebnisse \\
\hline$\chi^{2} / \mathrm{df}$ & $\leq 2,0$ & $59 / 62=0,95$ \\
\hline RMSEA & $\leq 0,05$ & 0,00 \\
\hline SRMR & $\leq 0,05$ & 0,04 \\
\hline GFI & $\geq 0,9$ & 0,94 \\
\hline AGFI & $\geq 0,9$ & 0,92 \\
\hline CFI & $\geq 0,97$ & 1,00 \\
\hline TLI & $\geq 0,97$ & 1,00 \\
\hline
\end{tabular}

Tabelle 24: Anpassungsgüte des Kovarianzstrukturmodells

444 Das hier dargestellte Strukturmodell folgt der in Abschnitt C3.5 eingeführten Gleichung (1). 
Generation zeigt (vgl. Tabelle 24). Somit kann konstatiert werden, dass ein sehr guter Fit der empirischen Daten mit dem spezifizierten Modell vorliegt.

Im nächsten Schritt kann daher die Überprüfung der einzelnen Strukturhypothesen anhand der Ergebnisse der Parameterschätzung - diese sind in Abbildung 28 dargestellt - erfolgen.

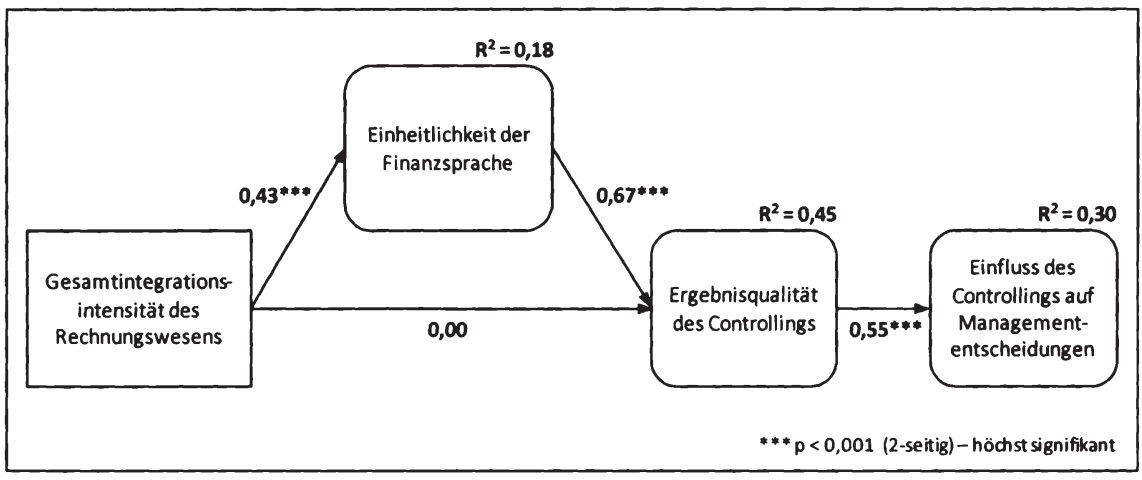

Abbildung 28: Ergebnisse der Parameterschätzung

Für die Gesamtintegrationsintensität des Rechnungswesens ergibt sich kein signifikanter direkter Effekt auf die Ergebnisqualität des Controllings. Die Hypothese $\mathrm{H}_{1}$ kann folglich nicht bestätigt werden. Des Weiteren zeigt sich, dass die Gesamtintegrationsintensität des Rechnungswesens - in Übereinstimmung mit Hypothese $\mathrm{H}_{2}$ - einen höchst signifikanten direkten Effekt $(0,43)$ auf die Einheitlichkeit der Finanzsprache besitzt. Wie der $\mathrm{R}^{2}$-Wert erkennen lässt, werden durch diesen positiven Einfluss $18 \%$ der Varianz der Variablen 'Einheitlichkeit der Finanzsprache' erklärt. Diese wiederum hat - wie in Hypothese $\mathrm{H}_{3}$ postuliert - einen höchst signifikanten direkten Effekt $(0,67)$ auf die Ergebnisqualität des Controllings, welcher $45 \%$ der Varianz dieser abhängigen Variablen ausmacht. Darüber hinaus wirkt die Ergebnisqualität des Controllings mit einem ebenfalls höchst signifikanten direkten Effekt $(0,55)$ positiv auf den Einfluss des Controllings auf Managemententscheidungen, wodurch $30 \%$ der Varianz dieser Variablen erklärt werden. Somit kann Hypothese $\mathrm{H}_{4}$ angenommen werden.

Die Ergebnisse zeigen, dass die technische Ausgestaltung der Controllerarbeit offensichtlich nicht in direkter Weise bedeutsam für die Beurteilung der Ergebnisqualität des Controllings seitens der Manager ist. Vielmehr ist hierfür ausschlaggebend, 
inwieweit es den Controllern gelingt, ein zu den Informationen der Bilanzierung konsistentes Bild der Geschäftssituation im Sinne einer einheitlichen Finanzsprache zu vermitteln. Hierauf wiederum hat die Gesamtintegrationsintensität des Rechnungswesens einen positiven Einfluss.

Zur genaueren Untersuchung der Dependenzbeziehungen - insbesondere die Quantifizierung und Signifikanzprüfung des mediierenden Effektes einer einheitlichen Finanzsprache steht hierbei im Vordergrund - erfolgt im nächsten Schritt eine Effektzerlegung in direkte, indirekte und Gesamteffekte mithilfe der Methode der so genannten 'bias-corrected bootstrap confidence intervals'. ${ }^{445}$ Die Ergebnisse dieser Effektzerlegung in Tabelle 25 belegen, dass der indirekte Effekt in Höhe von 0,29 $(=0,43 * 0,67)$, der von der Gesamtintegrationsintensität des Rechnungswesens über die Einheitlichkeit der Finanzsprache auf die Ergebnisqualität des Controllings wirkt, statistisch höchst signifikant ist. Da der direkte Effekt der Gesamtintegrationsintensität des Rechnungswesens auf die Ergebnisqualität des Controllings zudem 0,00 beträgt, liegt eine vollständige Mediation der Dependenzbeziehung dieser beiden Variablen durch die Einheitlichkeit der Finanzsprache vor. Entfernt man zu Vergleichs- bzw. Überprüfungszwecken die Variable 'Einheitlichkeit der Finanzsprache' aus dem Untersuchungsmodell, so ergibt sich zwar ein hoch signifikanter direkter Effekt der Gesamtintegration des Rechnungswesens auf die Ergebnisqualität des Controllings, der ebenfalls 0,29 beträgt. Der Erklärungsgehalt der letztgenannten Variablen liegt in diesem Falle jedoch lediglich bei $\mathbf{8 \%}$ und fällt somit deutlich geringer aus als im Falle des vollständigen Ausgangsmodells mit $45 \%$. Hierin ist - neben der Tatsache, dass bei Vorhandensein der Variablen 'Einheitlichkeit der Finanzsprache' der genannte direkte Effekt gänzlich aufgehoben wird - ein weiterer Beleg für die vollständige Mediation der Dependenzbeziehung zu sehen. ${ }^{446}$

$445 \mathrm{Zu}$ dieser Variante des Bootstrapping sowie zur Effektzerlegung vgl. die Ausfuhrungen in Abschnitt C3.5 der vorliegenden Arbeit.

Vgl. Wood et al. (2008), S. 274. 


\begin{tabular}{|c|c|c|c|c|c|c|c|c|c|}
\hline & \multicolumn{3}{|c|}{ Direkte Effekte } & \multicolumn{3}{|c|}{ Indirekte Effekte } & \multicolumn{3}{|c|}{ Gesamteffekte } \\
\hline $\begin{array}{l}\text { Abhängige } \\
\text { Variablen } \\
\text { Unab- } \\
\text { hängige } \\
\text { Variablen }\end{array}$ & 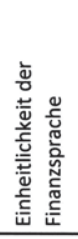 & 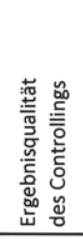 & 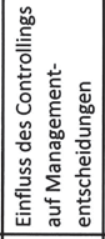 & 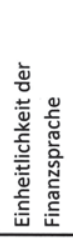 & 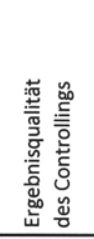 & 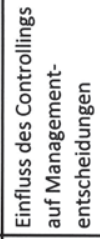 & 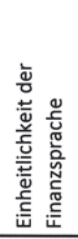 & 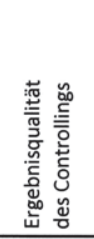 & 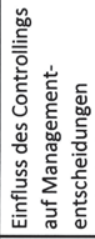 \\
\hline \begin{tabular}{|l|} 
Gesamtintegrations- \\
intensität des \\
Rechnungswesens \\
\end{tabular} & $0,43^{* *}$ & 0,00 & & & $0,29 * * *$ & $0,16^{* * *}$ & $0,43^{* *}$ & $0,29 * *$ & $0,16^{* * *}$ \\
\hline $\begin{array}{l}\text { Einheitlichkeit der } \\
\text { Finanzsprache }\end{array}$ & & $0,67^{* * *}$ & & & & $0,37^{* * *}$ & & $0,67^{* * *}$ & $0,37^{* * *}$ \\
\hline $\begin{array}{l}\text { Ergebnisqualität } \\
\text { des Controllings }\end{array}$ & & & $0,55^{* * *}$ & & & & & & $0,55^{* * *}$ \\
\hline
\end{tabular}

Tabelle 25: Ergebnisse der Effektzerlegung

Aus dem soeben erwähnten mediierenden Effekt ergibt sich in Kombination mit dem höchst signifikanten direkten Einfluss der Ergebnisqualität des Controllings auf den Einfluss des Controllings auf Managemententscheidungen $(0,55)$ zudem ein ebenfalls höchst signifikanter positiver Gesamteffekt der Gesamtintegrationsintensität des Rechnungswesens auf den Einfluss des Controllings auf Managemententscheidungen in Höhe von $0,16(=0,55 * 0,29)$.

Es kann folglich konstatiert werden, dass die Gesamtintegrationsintensität des Rechnungswesens den Controllingerfolg - sowohl hinsichtlich des Outputs als auch des Outcome der Controllerarbeit - positiv beeinflusst. Der Einheitlichkeit der Finanzsprache kommt in diesem Zusammenhang eine besondere Bedeutung $\mathrm{zu}$, da sie die Dependenzbeziehung der beiden genannten Variablen vollständig mediiert und somit offenbar einen 'missing link' innerhalb der Kausalkette darstellt.

Die bisherigen Ergebnisse der Kovarianzstrukturanalyse zeigen zudem, dass die mit einer Integration des Rechnungswesens assoziierten Chancen bzw. Vorteile - diese wurden insbesondere in einer verbesserten Kommunikation sowie einer erhöhten Verständlichkeit und Transparenz gesehen $-{ }^{447}$ tatsächlich in Form einer einheitliche- 
ren Finanzsprache auftreten und hierdurch zu einer positiveren Beurteilung des Erfolgs der Controllerarbeit seitens des Managements führen. ${ }^{448}$

Als weiterer Untersuchungsschritt wird eine Detailanalyse der Treiber der soeben dargestellten positiven Gesamtwirkung der Integration des Rechnungswesens auf den Erfolg der Controllerarbeit durchgeführt. Hierzu wird der Gesamtintegrationsindex nacheinander durch die fünf aktionsfeldbezogenen Integrationssubindizes ersetzt. ${ }^{449}$ Auf diese Weise ergeben sich fün separate Kovarianzstrukturmodelle, die alle das in Tabelle 24 ersichtliche Anspruchsniveau hinsichtlich der globalen Gütekriterien der zweiten Generation erfüllen. Eine Übersicht über die aus den Modellen resultierenden direkten, indirekten und Gesamteffekte der Integrationssubindizes auf die drei endogenen latenten Variablen bietet die nachfolgende Tabelle 26.

Die Effektzerlegung offenbart, dass die Integration des Rechnungswesens innerhalb der einzelnen Aktionsfelder der Controllerarbeit von unterschiedlicher Bedeutung für den Erfolg der Controllerarbeit ist. So zeigt sich, dass lediglich die Integration des Rechnungswesens innerhalb der Aktionsfelder 'Berichtswesen' (0,24 bzw. 0,13), 'Gestaltung der controllingrelevanten IT-Systeme' $(0,21$ bzw. 0,12$)$ und 'Institutionalisierung der Controllerarbeit' $(0,23$ bzw. 0,13$)$ einen statistisch - teilweise hoch bzw. höchst - signifikanten Gesamteffekt auf die Ergebnisqualität des Controllings sowie auf den Einfluss des Controllings auf Managemententscheidungen besitzt. Die Integration des Rechnungswesens innerhalb der Planung sowie der Performance-Messung ist hingegen ohne Bedeutung für die Beurteilung des Outputs bzw. des Outcome der Controllerarbeit seitens des Managements. Zwar ergibt sich für beide Integrationssubindizes zunächst ein jeweils signifikanter, wenngleich auch verhältnismäßig schwacher indirekter Effekt auf die Ergebnisqualität des Controllings (0,12 bzw. 0,10). Da bei der Berechnung des Gesamteffektes allerdings auch die direkten Effekte mit einfließen diese weisen in beiden Fällen ein negatives Vorzeichen auf ( $-0,05$ bzw. $-0,10)$, wenn-

Zu einem ăhnlichen Ergebnis kommt Weide (2009) in seiner empirischen Studie. Dort geben 96\% der Unternehmen an, dass die Integration des Rechnungswesens zu einer Erhöhung der Transparenz im Management Reporting geführt hat, womit die gesteigerte Transparenz in diesem $\mathrm{Zu}-$ sammenhang zugleich den wichtigsten Erfolgsindikator des Management Reportings bildet, vgl. Weide (2009), S. 315-317 sowie ergănzend die Ausführungen in Abschnitt B4 der vorliegenden Arbeit.

$449 \mathrm{Zu}$ den Integrationssubindizes vgl. die Ausführungen in Abschnitt D2 der vorliegenden Arbeit. 
gleich sie jedoch nicht signifikant sind - ergibt sich hier in beiden Fällen insgesamt kein signifikanter Einfluss auf den Controllingerfolg.

\begin{tabular}{|c|c|c|c|c|c|c|c|c|c|}
\hline & \multicolumn{3}{|c|}{ Direkte Effekte } & \multicolumn{3}{|c|}{ Indirekte Effekte } & \multicolumn{3}{|c|}{ Gesamteffekte } \\
\hline $\begin{array}{l}\text { Abhängige } \\
\text { Unab- } \\
\text { hängige } \\
\text { Variablen: } \\
\text { Integrations- } \\
\text { subindizes }\end{array}$ & 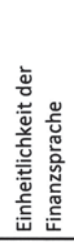 & 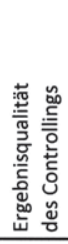 & 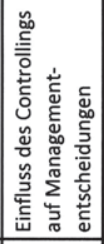 &  & 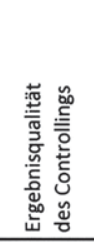 & 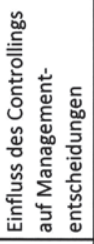 & 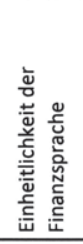 & 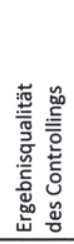 & 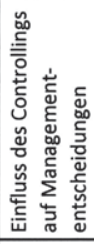 \\
\hline Planung & $0,17^{*}$ & $-0,05$ & & & $0,12^{*}$ & 0,04 & $0,17^{*}$ & 0,07 & 0,04 \\
\hline Berichtswesen & $0,41^{* *}$ & $-0,04$ & & & $0,29 * * *$ & $0,13^{* * *}$ & $0,41^{* *}$ & $0,24^{* *}$ & $0,13^{* * *}$ \\
\hline $\begin{array}{l}\text { Performance- } \\
\text { Messung }\end{array}$ & 0,15 & $-0,10$ & & & $0,10^{*}$ & 0,00 & 0,15 & 0,01 & 0,00 \\
\hline $\begin{array}{l}\text { Gestaltung der } \\
\text { controllingrele- } \\
\text { vanten IT-Systeme }\end{array}$ & $0,23^{*}$ & 0,06 & & & $0,15^{*}$ & $0,12^{*}$ & $0,23^{*}$ & $0,21^{*}$ & $0,12^{*}$ \\
\hline $\begin{array}{l}\text { Institutionalisierung } \\
\text { der Controllerarbeit }\end{array}$ & 0,16 & 0,13 & & & 0,10 & $0,13^{* * *}$ & 0,16 & $0,23^{* *}$ & $0,13^{* * *}$ \\
\hline
\end{tabular}

* $p<0,05$ - signifikant (two-tailed; bias-corrected bootstrap confidence intervals) ** $p<0,01$ - hoch signifikant (two-tailed; bias-corrected bootstrap confidence intervals) *** $p<0,001$ - höchst signifikant (two-tailed; bias-corrected bootstrap confidence intervals)

Tabelle 26: Detailanalyse der aktionsfeldbezogenen Submodelle

Im Falle der Erfolgswirkung des Subindexes 'Institutionalisierung der Controllerarbeit' wird die in Abschnitt C3.5 angesprochene methodische Überlegenheit deutlich, den die kovarianzstrukturanalytische Signifikanzprüfung von indirekten bzw. Gesamteffekten mithilfe des Bootstrapping-Verfahrens mit sich bringt. Obwohl neben dem direkten Effekt auf die Einheitlichkeit der Finanzsprache $(0,16)$ auch der direkte Effekt dieses Subindexes auf die Ergebnisqualität des Controllings $(0,13)$ die Signifikanzschwelle knapp verfehlt (die p-Werte liegen hier bei 0,09 bzw. 0,11), ergibt sich dennoch ein hoch signifikanter Gesamteffekt auf die Ergebnisqualität des Controllings $(0,23)$.

Eine weitere aufschlussreiche Erkenntnis liefert die Detailanalyse hinsichtlich der Wirkung der aktionsfeldbezogenen Integrationssubindizes auf die Einheitlichkeit der Finanzsprache. Hier zeigt sich, dass von der Integration des Rechnungswesens innerhalb des Berichtswesens - im Vergleich zu den Subindizes der übrigen Aktionsfelder - 
mit Abstand der stärkste Effekt $(0,41)$ auf die Konsistenz der verfügbaren Finanzinformationen im Unternehmen ausgeht. Entsprechend ist auch der aus diesem Integrationsbereich resultierende indirekte Effekt auf die Ergebnisqualität des Controllings mit einem Wert in Höhe von 0,29 am stärksten ausgeprägt.

Abschließend ist somit festzuhalten, dass die Wirkung einer Integration des Rechnungswesens auf den Controllingerfolg differenziert zu beurteilen ist, da diesbezüglich lediglich für die Aktionsfelder 'Berichtswesen', 'Gestaltung der controllingrelevanten IT-Systeme' und 'Institutionalisierung der Controllerarbeit' ein positiver Gesamteffekt zu verzeichnen ist.

\subsection{Mehrgruppenanalyse zur Identifizierung eines moderierenden Effektes der Gruppenzugehörigkeit der Antwortenden auf die Dependenzbeziehungen}

Im nächsten Analyseschritt soll in explorativer Weise untersucht werden, ob es hinsichtlich der Überprüfung der Hypothesen $\mathrm{H}_{1}$ bis $\mathrm{H}_{4}$ des obigen Untersuchungsmodells zu abweichenden Ergebnissen zwischen den befragten Controllern der dyadischen Stichprobe einerseits und den zugehörigen Managern andererseits kommt. Zum Nachweis eines solchen eventuell vorhandenen moderierenden Effektes der Gruppenzugehörigkeit der Antwortenden auf die Ausprägung der betrachteten Dependenzbeziehungen wird eine kovarianzstrukturanalytische Mehrgruppenanalyse durchgeführt. ${ }^{450}$ Innerhalb der ersten Gruppe wird das Untersuchungsmodell hierbei erneut entsprechend der Abbildung 25 abgebildet. Die exogene Variable basiert folglich auf den Antworten der Controller, während die endogenen Variablen anhand der Antworten der korrespondierenden Manager gemessen werden. Für die zweite Gruppe erfolgt hingegen die Messung sämtlicher Variablen des Untersuchungsmodells - d.h. sowohl der exogenen als auch der endogenen Variablen - auf Basis der Antworten der Controller der dyadischen Stichprobe. ${ }^{451}$

450 Vgl. hierzu die Ausfuhrungen in Abschnitt C3.5 der vorliegenden Arbeit.

451 Der einzige Unterschied hinsichtlich der Operationalisierung der betrachteten latenten Variablen im Rahmen der Mehrgruppenanalyse liegt darin, dass zur Gewăhrleistung eines guten Modellfits für beide Vergleichsgruppen bei der Messung des Konstrukts 'Ergebnisqualität des Controllings' das fünfte Item - vgl. hierzu Tabelle 17 - eliminiert wurde. 
Für die so abgegrenzten Gruppen wird nun simultan eine unabhängige Schätzung eines jeweils eigenständigen Modells durchgeführt. ${ }^{452}$ Die $\chi^{2}$-Statistik dieser Modellschätzung sowie die hieraus für beide Gruppen resultierenden standardisierten Effekte des Strukturmodells sind in Tabelle 27 ersichtlich.

\begin{tabular}{|c|c|c|c|c|c|}
\hline \multirow[b]{2}{*}{ Untersuchter Effekt } & \multicolumn{3}{|c|}{$\begin{array}{l}\text { Unabhängige Schätzung des jeweils } \\
\text { untersuchten Effektes }\end{array}$} & \multirow{2}{*}{$\begin{array}{c}\chi^{2} \text {-Statistik bei } \\
\text { Schätzung des } \\
\text { jeweils } \\
\text { untersuchten } \\
\text { Effektes mit } \\
\text { Identitäts- } \\
\text { restriktion }\end{array}$} & \multirow[b]{2}{*}{$\begin{array}{c}\Delta \chi^{2}- \\
\text { Statistik }\end{array}$} \\
\hline & $\begin{array}{c}\text { Gruppe 1: } \\
\text { Endogene } \\
\text { Variablen } \\
\text { aus } \\
\text { Manager- } \\
\text { sicht }\end{array}$ & $\begin{array}{c}\text { Gruppe 2: } \\
\text { Endogene } \\
\text { Variablen } \\
\text { aus } \\
\text { Controller- } \\
\text { sicht }\end{array}$ & $\chi^{2}$-Statistik & & \\
\hline $\begin{array}{l}\text { Integrationsintensität des } \\
\text { Rechnungswesens } \\
\rightarrow \text { Ergebnisqualität des } \\
\text { Controllings }\end{array}$ & 0,01 & 0,11 & \multirow{4}{*}{117,33} & 117,84 & 0,51 \\
\hline $\begin{array}{l}\text { Integrationsintensität des } \\
\text { Rechnungswesens } \\
\rightarrow \text { Einheitlichkeit der } \\
\text { Finanzsprache }\end{array}$ & 0,42 & 0,47 & & 117,90 & 0,57 \\
\hline $\begin{array}{l}\text { Einheitlichkeit der Finanzsprache } \\
\rightarrow \text { Ergebnisqualität des } \\
\text { Controllings }\end{array}$ & 0,66 & 0,37 & & 124,91 & $7,58^{* *}$ \\
\hline $\begin{array}{l}\text { Ergebnisqualität des Controllings } \\
\rightarrow \text { Einfluss des Controllings auf } \\
\text { Managemententscheidungen }\end{array}$ & 0,55 & 0,59 & & 117,67 & 0,34 \\
\hline
\end{tabular}

$* * \Delta \chi^{2} \geq 6,63-$ hoch signifikant

Tabelle 27: Ergebnisse der kovarianzstrukturanalytischen Mehrgruppenanalyse zur Identifixierung eines moderierenden Effektes

Zur Überprüfung, ob es sich bei den zwischen den Gruppen diesbezüglich aufgetretenen Abweichungen tatsächlich um das Ergebnis eines moderierenden Effektes handelt, werden als nächstes sukzessive vier restringierte Kovarianzstrukturmodelle spezifiziert. Hierbei wird für den jeweils betrachteten $\gamma$ - bzw. $\beta$-Koeffizienten eine Identitätsrestriktion eingeführt, sodass für diesen somit nur noch eine einmalige Schät- 
zung über beide Gruppen hinweg erforderlich ist. Sollte die restringierte Schätzung des interessierenden Effektes zu einer signifikanten Modellverschlechterung führen, so ist das Vorhandensein eines moderierenden Effektes nachgewiesen.

Wie die $\Delta \chi^{2}$-Statistik - ebenfalls in Tabelle 27 - zeigt, kommt es im Falle der dritten betrachteten Dependenzbeziehung aufgrund der restringierten Schätzung zu einer Differenz der $\chi^{2}$-Werte in Höhe 7,58. Diese liegt über dem kritischen Wert von $6,63^{453}$ und belegt somit eine hoch signifikante Modellverschlechterung. ${ }^{454}$ Daher kann konstatiert werden, dass ein moderierender Effekt der Gruppenzugehörigkeit der Antwortenden auf den zuvor durch die Hypothese $\mathrm{H}_{3}$ erfassten Einfluss der Einheitlichkeit der Finanzsprache auf die Ergebnisqualität des Controllings besteht. ${ }^{455}$ Dieser moderierende Effekt ist nicht nur hoch signifikant, sondern auch absolut betrachtet als sehr bedeutsam einzustufen, da der sich bei der ersten Gruppe der Manager ergebende Einfluss der Einheitlichkeit der Finanzsprache auf die Ergebnisqualität des Controllings mit 0,66 fast doppelt so hoch ausfällt wie im Controllermodell mit 0,37. Die Bedeutung, die die Controller der Einheitlichkeit der Finanzsprache im Hinblick auf den Controllingerfolg beimessen, ist somit deutlich geringer als aus Sicht der Manager.

Offensichtlich gehen die befragten Controller der dyadischen Stichprobe im Vergleich zu den korrespondierenden Managern verstärkt davon aus, dass andere, nicht im Untersuchungsmodell erfasste Einflussfaktoren auf die Ergebnisqualität des Controllings existieren und somit für die Beurteilung des Controllererfolgs relevant sind. Dies unterstreicht der Vergleich der sich aus der unabhängigen Mehrgruppenmodellschätzung ergebenden jeweiligen erklärten Varianz des Konstrukts 'Ergebnisqualität des Controllings'. Während der diesbezügliche $\mathrm{R}^{2}$-Wert für die erste Gruppe der Manager 0,44 beträgt, fällt der entsprechende Wert im Falle des Controllermodells mit lediglich 0,19 wesentlich geringer aus.

Bei einer Erhöhung um einen Freiheitsgrad ergeben sich die folgenden kritischen Werte auf Basis der $\chi^{2}$-Verteilung: $\Delta \chi^{2} \geq 3,84$ (5\%-Niveau - signifikant); $\Delta \chi^{2} \geq 6,63$ (1\%-Niveau - hoch signifikant); $\Delta \chi^{2} \geq 10,83(0,1 \%$-Niveau - höchst signifikant).

Dennoch weist dieses restringierte Mehrgruppenmodell - bei einem p-Wert von 0,07 - weiterhin eine hohe Anpassungsgüte auf. So beträgt das Verhältnis $\chi^{2} / \mathrm{df}$ beispielsweise $1,21(=124,91 / 103)$, der RMSEA 0,03 und der CFI sowie der TLI jeweils 0,99 . Effekt vor, da die jeweilige Identitätsrestriktion zu keiner signifikanten Modellverschlechterung fuhrt. 
Aus dem Ergebnis der kovarianzstrukturanalytischen Mehrgruppenanalyse können auch Rückschlüsse auf die Qualität der Informantenangaben gezogen werden. ${ }^{456}$ So deutet der hoch signifikante Unterschied bezüglich der von der Einheitlichkeit der Finanzsprache auf die Ergebnisqualität des Controllings wirkenden Effektstärke auf einen systematischen Beurteilungsunterschied zwischen den Controllern einerseits und den Managern andererseits hin. Eine solche informantenbedingte Verzerrung wird als Key Informant Bias bezeichnet. ${ }^{457} \mathrm{Zu}$ dessen zentralen Ursachen zählt - neben Aspekten wie abweichenden Motiven, beschränkten Informationsverarbeitungskapazitäten oder divergierenden Informationsständen - die Subjektivität der Wahrnehmung auf Seiten der jeweiligen Schlüsselinformanten ${ }^{458} .459$ Ein wichtiger Treiber für das Auftreten einer divergierenden Wahrnehmung ist dabei insbesondere die funktionale Zugehörigkeit der Antwortenden. ${ }^{460}$ In diesem Zusammenhang stellen Kumar/Stern/ Anderson (1993) fest, dass

„informants may disagree because they hold different organizational positions and thus different perspectives on the same organizational phenomena. "461

Treten - wie im vorliegenden Fall - innerhalb eines Validierungsdesigns systematische Wahrnehmungsunterschiede zwischen Informantengruppen auf, so kommt der Entscheidung eine besondere Bedeutung zu, auf wessen Angaben zur Messung welcher Variablen zurückgegriffen wird. Im Zuge dieser Auswahl sollten vor allem konzeptionelle Überlegungen bezüglich der Informanten ausschlaggebend sein. ${ }^{462}$

Vgl. Homburg/Klarmann (2009), S. 163.

Vgl. Ernst (2003), S. 1250, Homburg (2007), S. 43 sowie Homburg/Klarmann (2009), S. 149.

Als Schlüsselinformant wird eine Auskunftsperson bezeichnet, die Angaben uber Eigenschaften und Verhalten der Organisation macht, in der sie beschäftigt ist, vgl. Hurrle/Kieser (2005), S. 585, Homburg (2007), S. 43 sowie Homburg/Klarmann (2009), S. 149. Der Rückgriff auf Schlusselinformanten ist innerhalb der großzahligen empirischen Controllingforschung - ăhnlich wie in der Marketing-, Personal- und Organisationsforschung - weit verbreitet, vgl. Homburg (2007), S. 43.

Vgl. Ernst (2003), S. 1250, Hurrle/Kieser (2005), S. 589f. sowie Homburg/Klarmann (2009), S. 149. Der Key Informant Bias unterscheidet sich somit von dem in Abschnitt E2.1 der vorliegenden Arbeit erläuterten Common Method Bias, bei dem sich Verzerrungen daraus ergeben, dass für die Messung von unabhăngigen und abhăngigen Variablen auf dieselbe Informationsquelle zurückgegriffen wird.

Vgl. Ernst (2003), S. 1253f., Homburg (2007), S. 43 sowie Homburg/Klarmann (2009), S. 150.

Kumar/Stern/Anderson (1993), S. 1636.

Vgl. Ernst (2003), S. 1260 sowie Homburg/Klarmann (2009), S. 166. 
Bezogen auf das hier betrachtete Untersuchungsmodell erscheint eine Berücksichtigung der Controllerantworten zur Messung des Einflusses der Einheitlichkeit der Finanzsprache auf die Ergebnisqualität des Controllings vor diesem Hintergrund eher weniger geeignet. $\mathrm{Zu}$ begründen ist diese Einschätzung zum einen damit, dass die Controller die Einheitlichkeit bzw. Konsistenz der aus Controlling und Bilanzierung bereitgestellten Finanzinformationen aufgrund ihrer funktionalen Position im Unternehmen und der damit verbundenen Perspektive nur schwerlich verzerrungsfrei bewerten können, wohingegen sich die Manager durch eine gewisse kritische Distanz bzw. Unvoreingenommenheit $\mathrm{zu}$ beiden Funktionsbereichen auszeichnen. Zum anderen könnten die Controller aus Gründen der Selbstdarstellung geneigt sein, beschönigende Angaben zu machen - insbesondere hinsichtlich der Ergebnisqualität ihrer eigenen Arbeitsleistung. Beide genannten Aspekte steigern das Risiko eines Key Informant Bias. ${ }^{463}$ Gestützt wird diese Argumentation durch die empirischen Ergebnisse von Pierce/O'Dea (2003), die in ihrer explorativ konzipierten Untersuchung deutliche „preparer-user perception gaps ${ }^{\text {‘464 }}$ hinsichtlich der Qualität und Nutzung von Informationen zwischen den befragten Managern sowie den ebenfalls befragten Management Accountants feststellen. ${ }^{465}$

Zudem sind es letztendlich die Manager, die über die Verwendung der seitens des Controllings bereitgestellten Informationen zur Entscheidungsfindung und somit über den Erfolg der Controllerarbeit bestimmen. In diesem Zusammenhang merken Van der Stede/Young/Chen (2005) an:

,[...] some argue that subjective measures [...] provide a 'better' type of information because subjective beliefs are reality, at least in the eyes of the respondent [...]. Hence, subjective measures [...] are based on those aspects [...] that are most salient to the respondents, and thus, are most likely to shape their behaviors and guide their actions. “466

Vgl. Homburg/Klarmann (2009), S. 155.

Pierce/O'Dea (2003), S. 257.

Vgl. Pierce/O'Dea (2003), S. 282. Die Autoren geben diesbezüglich zu bedenken, ,[...] that some management accountants may deliberately inflate the importance of the information they produce. There is also a risk that respondents may lack any detailed knowledge or understanding of the types of information which their managers are using and simply report what is being produced within their own departments" (S. 261).

Van der Stede/Young/Chen (2005), S. 675. Hervorhebung im Original durch Kursivdruck. 
Vor diesem Hintergrund kann konstatiert werden, dass die Gruppe der Manager die aus konzeptioneller Sicht besser geeigneten Schlüsselinformanten darstellt, um die Auswirkungen einer Integration des Rechnungswesens hinsichtlich der Einheitlichkeit der Finanzsprache und des Controllingerfolgs aus Nutzerperspektive zu untersuchen.

\section{Implikationen der Untersuchungsergebnisse zum Einfluss der Integration des Rechnungswesens auf die Controllerarbeit}

Die obigen Ergebnisse zum Einfluss der Integration des internen und externen Rechnungswesens auf die rollenbezogene Ausgestaltung sowie den Erfolg der Controllerarbeit beinhalten eine Reihe von praxisbezogenen Implikationen.

Hinsichtlich der Ausübung der Controllerrollen wurde gezeigt, dass die Integration des Rechnungswesens die Entwicklung des Controllers in Richtung Business Partner fördert - der zentrale Hebel liegt hierbei im Bereich der controllingrelevanten ITSysteme. Zur Realisierung dieses angestrebten Rollenwandels ist es somit erforderlich, dass die Controller aktiv die Notwendigkeit einer integrierten und zentralisierten Systemlandschaft propagieren und deren Implementierung im Unternehmen forcieren.

Aus Controllersicht erscheint ein solches Engagement zum einen erstrebenswert, da die hiermit einhergehende zeitliche Entlastung hinsichtlich der Rolle als Methodenund Systemdienstleister ihnen eine Konzentration auf die von ihnen verstärkt angestrebte Rolle als betriebswirtschaftliche Berater ermöglicht und somit letztlich auch ihre Arbeitszufriedenheit steigert. Zum anderen - dies hat die Detailanalyse der aktionsfeldbezogenen Submodelle ergeben - führt eine verstärkte IT-Integration zu einer einheitlicheren Finanzsprache und folglich zu einer positiveren Beurteilung sowie stärkeren Verwendung der bereitgestellten Controllinginformationen seitens des Managements. Die Controller können auf diese Weise neben ihrer Arbeitszufriedenheit somit auch ihren Einfluss auf die Entscheidungsfindung im Unternehmen erhöhen.

Ein ebenfalls positiver indirekter Effekt auf den Erfolg der Controllerarbeit geht darüber hinaus von der Integration des Rechnungswesens innerhalb der Aktionsfelder 'Berichtswesen' und 'Institutionalisierung der Controllerarbeit' aus. Von entscheidender Bedeutung ist hierbei die Einheitlichkeit der Finanzsprache aus Sicht des Managements, da diese die Dependenzbeziehung zwischen der Gesamtintegrationsintensität des Rechnungswesens und der Ergebnisqualität des Controllings vollständig mediiert. 
Controller sollten demzufolge verstärkt auf die Konsistenz der von ihnen bereitgestellten Informationen mit den Informationen der externen Finanzberichterstattung achten. Dieser Aspekt ist umso bedeutender, da die Controller den Einfluss dieser Konsistenz auf die Beurteilung der Ergebnisqualität des Controllings selbst nicht so hoch einschätzen wie die Manager. Diese sind es jedoch, die im Zuge ihrer Entscheidungsfindung das Ausmaß der Berücksichtigung von Controllinginformationen - und somit in letzter Konsequenz den Erfolg der Controllerarbeit - bestimmen.

Aus dieser Erkenntnis resultieren weit reichende Implikationen auch für Bereiche, die nicht in direkter Weise mit der Integration des Rechnungswesens in Zusammenhang stehen. So erscheinen beispielsweise die von Stewart (1994) zur Ermittlung des Economic Value Added (EVA) vorgeschlagenen bis zu 164 Anpassungen der Rechnungslegungsdaten vor diesem Hintergrund problematisch. ${ }^{467}$ Zwar zeigt Weaver (2001) anhand einer Studie von 29 den EVA verwendenden Unternehmen, dass durchschnittlich nur 19 dieser so genannten 'conversions' vorgenommen werden. ${ }^{468}$ Dennoch ist diesbezüglich zu konstatieren, dass der EVA mit einer steigenden Anzahl von Anpassungen auf einem zunehmend eigenständig generierten Datengerüst basiert. Diese Vorgehensweise ist möglicherweise als kontraproduktiv einzustufen, da die Konsistenz bzw. Verständlichkeit einer auf diese Weise generierten Steuerungskennzahl reduziert und somit auch ihr Stellenwert eingeschränkt wird. Demzufolge sollte die Ermittlung von wertorientierten Steuerungskennzahlen einfach und übersichtlich gehalten werden. ${ }^{469}$

Die Einheitlichkeit der Finanzsprache ist daher in gewisser Weise mit der Einfachheit der Finanzsprache bzw. der Informationsversorgung innerhalb des Unternehmens verknüpft. Unterstützt wird diese auf eine konzeptionelle Informationsnutzung abstellende Sichtweise durch Äußerungen seitens der Unternehmenspraxis, die sich für

Vgl. Stewart (1994), S. 73 sowie grundlegend zum Economic Value Added Stewart (1991).

Das Minimum lag bei sieben, das Maximum bei 34 Anpassungen. Vgl. Weaver (2001), S. 58.

$\mathrm{Zu}$ einem ähnlichen Fazit kommt Weber (2009) auf Basis einer Befragung der Controllingleiter der DAX 30-Unternehmen. Das Instrument der wertorientierten Steuerung generiert demnach „aufgrund seiner Komplexităt erhebliche Implementierungsprobleme. Diese liegen weniger bei der 'technischen Umsetzung', obwohl sie auch dort in erheblichem Maße auftreten. Der Schwerpunkt der Probleme ist vielmehr bei der Nutzung des Instruments durch das Management festzustellen“ (S. 303). So kann ,diese hohe Komplexităt [...] die Verständlichkeit und Nachvollziehbarkeit der wertorientierten Steuerung für die Manager gefăhrden" (S. 301). Diese Problematik lässt sich am ehesten lösen, „wenn ein vergleichsweise einfaches wertorientiertes Steuerungskonzept [...] implementiert wird" (S. 301). 
einfache und verständliche Managementberichte aussprechen und darauf verweisen, dass zu komplexe Berichte ohnehin keine Beachtung finden. ${ }^{470}$ Ergebnisse einer empirischen Studie zeigen zudem, dass Manager zufriedener mit Kennzahlen sind, wenn sie diese aus einer Hand und in einem Bericht erhalten. ${ }^{471}$ Offensichtlich besteht auf Seiten der Manager demnach der Bedarf nach einer konsistenten und einfachen Informationsversorgung.

Einfachheit stellt darüber hinaus zugleich eine Determinante von Entscheidungsqualität dar. So stellen Watrin/Pott (2008) fest:

„Die Entscheidungsqualität auf Basis von Rechnungslegungsinformationen hängt wesentlich davon $a b$, wie 'einfach' die Verarbeitung und die Interpretation von Rechnungslegungsinformationen sind. “472

Zwar bezieht sich diese Aussage auf Analysten und somit auf Externe; sie kann aber analog auf die unternehmensinterne Sphäre der Informationsversorgung übertragen werden. Hayes (1983) gibt in diesem Zusammenhang zu bedenken, dass ein zu hohes Maß an Komplexität bzw. Präzision schädlich für eine rechnungslegungsbasierte Kommunikation ist. ${ }^{473}$ Meyer (2007) stellt zudem anhand des Beispiels einer Implementierung des EVA zur Unternehmenssteuerung fest, dass aufgrund der begrenzten kognitiven Fähigkeiten von Entscheidungsträgern ein Trade-off zwischen Genauigkeit und dem schnellen Erlernen eines solchen Konzeptes und somit der Entscheidungsgüte besteht. ${ }^{474}$ Insofern kommt der Einfachheit der Informationsversorgung, die indes wiederum explizit mit der Integration des Rechnungswesens in Verbindung gebracht wird, ${ }^{475}$ eine hohe Bedeutung $\mathrm{zu}$. Controllern obliegt in diesem Zusammenhang folglich die anspruchsvolle Aufgabe, in ihrer Kommunikation mit dem Management die richtige Balance zwischen Genauigkeit und Einfachheit zu finden.

Bezug nehmend auf den Titel der vorliegenden Arbeit kann abschließend konstatiert werden, dass die Integration des internen und externen Rechnungswesens einen Erfolgsfaktor für die Controllerarbeit bildet, da sie die Einheitlichkeit der Finanzspra- 
che erhöht und hierdurch die Ergebnisqualität sowie den Entscheidungseinfluss des Controllings steigert. Wie in früheren Studien gezeigt wurde, wirken eine erhöhte Ergebnisqualität des Controllings bzw. ein erhöhter Einfluss des Controllings auf Managemententscheidungen zudem positiv auf den Unternehmenserfolg. So kommt Bauer (2002) in seiner Kovarianzstrukturanalyse zu dem Ergebnis, dass die Ergebnisqualität des Controllings einen hoch signifikanten standardisierten Effekt auf den Unternehmenserfolg in Höhe von 0,32 ausübt. Darüber hinaus stellt er eine hoch signifikante Korrelation zwischen dem Einfluss der Controller und dem Unternehmenserfolg in Höhe von 0,18 fest. ${ }^{476}$ Vor diesem Hintergrund stellt die Integration des Rechnungswesens womöglich einen Erfolgsfaktor nicht nur für die Controllerarbeit, sondern auch für den Unternehmenserfolg dar - wenngleich dieser Zusammenhang aufgrund der Vielzahl von Einflussfaktoren vermutlich empirisch nur schwer erfassbar sein dürfte.

Vgl. Bauer (2002), S. 252f. Zudem ermittelt Spillecke (2006) anhand eines PLS-basierten Strukturgleichungsmodells sowohl einen hoch signifikanten positiven Effekt der Ergebnisqualităt des Controllings $(0,41)$ als auch des Einflusses des Controllerbereichs $(0,17)$ auf die Qualităt des Führungszyklus, anhand derer er den Erfolg des Controllerbereichs festmacht. Die Qualität des Führungszyklus wiederum hat eine - ebenfalls hoch signifikante - positive Wirkung auf die Anpassungsfähigkeit $(0,34)$ sowie auf den kunden- bzw. marktbezogenen Erfolg des Unternehmens $(0,17$ bzw. 0,20$)$, vgl. Spillecke (2006), S. 185. Einen - eher allgemein gehaltenen - positiven Einfluss einer erfolgreichen Controllerarbeit auf den Unternehmenserfolg weisen zudem Weber et al. (2006) in ihrer Studie unter 618 Mitgliedern des Internationalen Controller Vereins (ICV) nach. Demnach weisen knapp zwei Drittel $(64,5 \%)$ der als erfolgreich eingestuften Unternehmen auch einen erfolgreichen Controllerbereich auf, während dies nur bei $35,5 \%$ der nicht als erfolgreich eingestuften Unternehmen der Fall ist, vgl. Weber et al. (2006), S. 68f. In ihrer unter 17 italienischen Großunternehmen durchgeführten Untersuchung stellen Zoni/Merchant (2007) zudem eine signifikante positive Korrelation zwischen dem Controllerinvolvement im Rahmen der Entscheidungsfindung und der Unternehmensperformance in Höhe von 0,58 fest, vgl. Zoni/Merchant (2007), S. 39. 


\section{F Schlussbetrachtung}

\section{Zusammenfassung der zentralen Ergebnisse}

Die Integration des internen und externen Rechnungswesens ist ein Praxisphänomen, welches im Laufe der letzten 15 Jahre - auch innerhalb der Wissenschaft - zunehmend an Bedeutung bzw. Aufmerksamkeit gewonnen hat. Das Ziel der vorliegenden Arbeit war es, den bislang eingeschränkten empirischen Erkenntnisstand innerhalb dieses Themenfelds auf Basis deskriptiv-explorativer sowie explanativer Analysen zu erweitern. Konkret sollte - ausgehend von einer umfassenden Ermittlung des Status quo der Integration des Rechnungswesens - der Einfluss der Integration auf die rollenbezogene Ausgestaltung und den Erfolg der Controllerarbeit untersucht werden.

Hierzu wurde eine fragebogengestützte Erhebung unter den 1.500 umsatzstärksten Unternehmen Deutschlands durchgeführt. Um die Nutzerperspektive des Managements hinsichtlich der im Unternehmen bereitgestellten Finanzinformationen valide abbilden zu können, wurde die Untersuchung dyadisch konzipiert. Dies bedeutet, dass neben dem Leiter Controlling jeweils auch ein ergebnisverantwortlicher Manager aus demselben Unternehmen befragt wurde.

Die zentralen Untersuchungsergebnisse sollen nachfolgend entlang der drei im Rahmen der Einleitung abgeleiteten Forschungsfragen zusammenfassend dargestellt werden.

(Ad) Forschungsfrage 1: Wie ist der Status quo der Integration des internen und externen Rechnungswesens in deutschen Großunternehmen?

Zur Ermittlung des Status quo wurden 17 integrationsrelevante Indikatoren erfasst und zu einem Gesamtintegrationsindex sowie zu fünf Integrationssubindizes - einem für jedes Aktionsfeld der Controllerarbeit - aggregiert. Die Indizes spiegeln dabei die Integrationsintensität des internen und externen Rechnungswesens innerhalb des jeweils betrachteten Bereichs wider und bewegen sich innerhalb des Wertebereichs von 0 und 5.

Wie die Analyse ergeben hat, ist die Integration des Rechnungswesens bei den Unternehmen der Hauptstichprobe insgesamt stark ausgeprägt. So weisen knapp drei 
Viertel (74,30\%) der Unternehmen einen Gesamtintegrationsindex von mindestens 3,5 und somit einen hohen bis sehr hohen Integrationsgrad auf. Bei den entsprechenden Subindizes ist dabei innerhalb des Aktionsfelds 'Gestaltung der controllingrelevanten IT-Systeme' lediglich ein vergleichsweise geringerer $(58,41 \%)$ und innerhalb des Aktionsfelds 'Institutionalisierung der Controllerarbeit' ein vergleichsweise höherer (81,78\%) Anteil der Unternehmen durch ein hohes bis sehr hohes Integrationsniveau gekennzeichnet.

Die ebenfalls durchgeführte Bildung kontextspezifischer Integrationsprofile hat zudem aufgezeigt, dass jeweils bei denjenigen Unternehmen eine signifikant stärkere Gesamtintegrationsintensität des Rechnungswesens vorliegt, die börsennotiert sind, die gemäß ihrer Mitarbeiterzahl von mehr als 10.000 als 'groß' eingestuft wurden, die die IFRS (statt der HGB-Vorschriften) als führenden Rechnungslegungsstandard verwenden oder die einer geringen Volatilität ihres operativen Ergebnisses unterliegen.

Ein weiterer Bestandteil der Status-quo-Ermittlung lag in der Identifizierung und Quantifizierung des Musters einer partiellen Integration des Rechnungswesens. Exemplarisch für die Fragestellung der operativen Planung konnte hier zunächst eine mit abnehmenden Hierarchiestufen sinkende durchschnittliche Integrationsintensität des Rechnungswesens nachgewiesen werden. In der anschließenden Detailanalyse wurden die partielle Integration sowie die einheitlich starke Integration als die beiden dominierenden Integrationsmuster - 42,78\% bzw. 42,20\% der untersuchten Unternehmen sind hierdurch gekennzeichnet - im Bereich der operativen Ergebnisplanung ermittelt. Überdurchschnittlich häufig angewendet werden beide Integrationsmuster von Unternehmen, die börsennotiert sind oder deren führenden Rechnungslegungsstandard die IFRS bilden.

(Ad) Forschungsfrage 2: Welchen Einfluss hat die Integration des Rechnungswesens auf die Ausübung unterschiedlicher Controllerrollen?

Zur Beantwortung der zweiten Forschungsfrage wurden zunächst die seitens der Controller bisher realisierte sowie die zukünftig angestrebte Ausübung der unterschiedlichen Rollenbilder des House of Controlling ermittelt. Die Ergebnisse zeigen, dass die Controller ihre Rolle als betriebswirtschaftlicher Berater des Managements von derzeit durchschnittlich $40,15 \%$ auf $53,10 \%$ signifikant ausweiten und im Gegen- 
zug insbesondere ihre Rolle als Methoden- und Systemdienstleister von bisher 38,23\% auf 28,81\% ihres zeitlichen Arbeitsaufwands signifikant reduzieren wollen.

Hierauf aufbauend konnten anschließend die Hypothesen bestätigt werden, dass die Controller derjenigen Unternehmen, die eine im Vergleich zum Median höhere Gesamtintegrationsintensität des Rechnungswesens aufweisen, die Rolle als betriebswirtschaftlicher Berater des Managements in einem signifikant höheren Ausmaß (45,21\% im Vergleich zu 35,86\%) und die Rolle als Methoden- und Systemdienstleister in einem signifikant geringeren Ausmaß (34,14\% im Vergleich zu 41,70\%) wahrnehmen. Bei der Betrachtung der aktionsfeldbezogenen Integrationssubindizes hat sich zudem gezeigt, dass für diesen Zusammenhang einzig die Integrationsintensität im Bereich 'Gestaltung der controllingrelevanten IT-Systeme' ausschlaggebend ist. Offensichtlich werden die Controller erst durch den Rückgriff auf eine integrierte und zentralisierte Systemlandschaft in ausreichendem Maße von ihrer Rolle als Methodenund Systemdienstleister entlastet, sodass eine Fokussierung auf die Beraterrolle und damit die Entwicklung zum Business Partner möglich wird.

Zusammenfassend lässt sich demnach festhalten, dass die Integration des Rechnungswesens im Bereich der controllingrelevanten IT-Systeme den seitens der Controller angestrebten Rollenwandel in Richtung betriebswirtschaftlicher Berater des Managements begünstigt.

(Ad) Forschungsfrage 3: Welchen Einfluss hat die Integration des Rechnungswesens auf den Erfolg der Controllerarbeit und welche Bedeutung kommt der Einheitlichkeit der Finanzsprache in diesem $\mathrm{Zu}$ sammenhang zu?

Im Rahmen der dritten Forschungsfrage wurde untersucht, inwieweit sich die Gesamtintegrationsintensität des Rechnungswesens auf den seitens des Managements beurteilten Erfolg der Controllerarbeit - dieser wurde anhand der Ergebnisqualität des Controllings (Output) sowie des Einflusses des Controllings auf Managemententscheidungen (Outcome) festgemacht - auswirkt. Hierbei wurde die verhaltenswissenschaftlich geprägte Sichtweise von Finanzinformationen des Rechnungswesens als Sprache bzw. Kommunikationsmedium explizit in Form der Variablen 'Einheitlichkeit der Finanzsprache' im Untersuchungsmodell berücksichtigt. 
Im Zuge der auf Basis der dyadischen Stichprobe durchgeführten Kovarianzstrukturanalyse konnte kein signifikanter direkter Effekt der Integrationsintensität des Rechnungswesens auf die Ergebnisqualität des Controllings ermittelt werden; der Pfadkoeffizient betrug hier 0,00 . Hingegen ergaben sich jeweils signifikant positive Effekte der Gesamtintegrationsintensität des Rechnungswesens auf die Einheitlichkeit der Finanzsprache (0,43), der Einheitlichkeit der Finanzsprache auf die Ergebnisqualität des Controllings $(0,67)$ und der Ergebnisqualität des Controllings auf den Einfluss des Controllings auf Managemententscheidungen $(0,55)$. Im Rahmen einer auf Bootstrapping-Basis durchgeführten Effektzerlegung konnte nachgewiesen werden, dass der sich hieraus ergebende - vollständig von der Einheitlichkeit der Finanzsprache mediierte - indirekte Effekt der Gesamtintegrationsintensität des Rechnungswesens auf den Erfolg der Controllerarbeit ebenfalls statistisch signifikant ist. Die von der Integration ausgehende Gesamtwirkung auf die Ergebnisqualität des Controllings bzw. auf den Einfluss des Controllings auf Managemententscheidungen wurde dabei mit 0,29 bzw. 0,16 beziffert. Hierdurch wurde empirisch belegt, dass die Integration des Rechnungswesens die Controller dabei unterstützt, Mitverantwortung für die Erreichung der Unternehmensziele im Sinne des Controllerleitbilds der International Group of Controlling zu übernehmen.

In der anschließend anhand der aktionsfeldbezogenen Integrationssubindizes vorgenommenen Detailanalyse wurde aufgezeigt, dass der positive Gesamteffekt der Integration auf die Ergebnisqualität des Controllings und den Einfluss des Controllings auf Managemententscheidungen ausschließlich auf das Berichtswesen, die Gestaltung der controllingrelevanten IT-Systeme sowie die Institutionalisierung der Controllerarbeit zurückzuführen ist. Von der Integration des Rechnungswesens innerhalb der Aktionsfelder 'Planung' und 'Performance Messung' geht hingegen keine signifikante Gesamtwirkung auf den Controllingerfolg aus.

Im Zuge einer kovarianzstrukturanalytischen Mehrgruppenanalyse konnte darüber hinaus offengelegt werden, dass die Controller die Bedeutung der Einheitlichkeit der Finanzsprache für die Beurteilung der Ergebnisqualität des Controllings im Vergleich zu den Managern unterschätzen und somit offenbar verstärkt von anderen, nicht im Untersuchungsmodell enthaltenen Einflussfaktoren auf den Controllingerfolg ausgehen. 


\section{Kritische Würdigung und Ausblick auf die weitere Forschung}

Die Ergebnisse der vorliegenden Arbeit tragen inhaltlich zu einem tieferen Verständnis der Gestaltungsparameter und Ausprägung einer Integration des internen und externen Rechnungswesens sowie deren Wirkung auf die Controllerarbeit bei. So wurde auf Basis einer großzahligen empirischen Studie die bislang umfassendste Status-quoErmittlung der Integration des internen und externen Rechnungswesens bei deutschen Großunternehmen durchgeführt. Hierauf aufbauend wurden zudem erstmals die Konsequenzen der Integrationsintensität für die rollenbezogene Ausgestaltung und den Erfolg der Controllerarbeit analysiert. Es konnte zum einen gezeigt werden, dass eine hohe Integrationsintensität des Rechnungswesens die Controller in ihrer Zielsetzung unterstïtzt, verstärkt als betriebswirtschaftlicher Berater des Managements zu fungieren. Zum anderen wurde eine positive indirekte Wirkung der Integration auf die Beurteilung und Verwendung der Controllinginformationen seitens des Managements nachgewiesen. Hinsichtlich des letztgenannten Aspekts stellt die Einheitlichkeit der Finanzsprache offensichtlich einen 'missing link' innerhalb der Kausalkette dar, da sie die Dependenzbeziehung zwischen der Integrationsintensität des Rechungswesens und der Ergebnisqualität des Controllings vollständig mediiert. Insbesondere, da die rein instrumentelle bzw. technische Sichtweise also keinen direkten Effekt ergibt, kommt der empirischen Berücksichtigung der verhaltenswissenschaftlich geprägten Perspektive von Rechnungslegungsinformationen als Sprache bzw. Kommunikationsinstrument eine hohe Bedeutung zu.

Dieser Ansatz wurde bisher im Kontext der Integration des Rechnungswesens noch nicht verfolgt und kann folglich als innovativ eingestuft werden. Er setzt die Forderung um,

„das Controlling viel stärker aus dem Blickwinkel der Auswirkungen auf das Führungsverhalten zu sehen, also die Brille eines 'Behavioral Controlling' aufzusetzen. " 477

In methodischer Hinsicht zeichnet sich die Analyse insbesondere durch das im Rahmen der Beantwortung der dritten Forschungsfrage zu Grunde gelegte dyadische Untersuchungsdesign aus, welches eine valide Berücksichtigung der Nutzerperspektive des Managements hinsichtlich der im Unternehmen bereitgestellten Finanzinforma- 
tionen zulässt. Darüber hinaus wurde mit der Kovarianzstrukturanalyse das derzeit wohl leistungsstärkste Verfahren zur Analyse von Dependenzbeziehungen innerhalb der empirischen Sozialforschung eingesetzt. Beide Aspekte bilden in Kombination ein Alleinstellungsmerkmal im relevanten Forschungsfeld. ${ }^{478}$

Trotz der Vorzüge des gewählten Ansatzes unterliegen die Untersuchungsergebnisse der vorliegenden Arbeit gewissen Limitationen, aus denen sich weiterer Forschungsbedarf ergibt.

So erfolgt die Analyse im Rahmen der ersten Forschungsfrage überwiegend und im Falle der zweiten und dritten Forschungsfrage ausschließlich hinsichtlich der obersten Hierarchieebene der befragten Unternehmen. Zum einen ist jedoch die Integrationsintensität des internen und externen Rechnungswesens auf unterschiedlichen Hierarchieebenen oftmals verschieden stark ausgeprägt - dies zeigen die Ergebnisse zur Identifizierung und Quantifizierung des Musters einer partiellen Integration in Abschnitt D3.1. Zum anderen unterscheiden sich die Informationsbedarfe seitens der Manager auf niedrigeren, stärker operativ geprägten Ebenen typischerweise von denen des Top-Managements. ${ }^{479}$ Folglich können die vorliegenden Analyseergebnisse nicht ohne weiteres auf niedrigere Hierarchieebenen übertragen werden. Zukünftige Untersuchungen könnten daher eine stärker leitungsebenendifferenzierte Analyse der Integrationswirkung auf die Managementzufriedenheit mit der Informationsversorgung durch das Controlling durchführen.

Die vorliegende Untersuchung wurde zudem branchenübergreifend durchgeführt. ${ }^{480}$ Branchenspezifische Auswertungen waren aufgrund der pro Branche geringen Fallzahl jedoch auf Basis der vorliegenden Stichprobe nicht möglich. In zukünftigen Studien könnte die Gültigkeit der ermittelten Kernergebnisse für spezifische Branchen überprüft werden. Auch könnte im Falle eines größeren Stichprobenumfangs in einer Replikationsstudie untersucht werden, inwieweit Kontextfaktoren, wie z.B. der ver-

Vgl. hierzu die Ausführungen über die bisherige empirische Forschung zur Integration des Rechnungswesens in Abschnitt B4 der vorliegenden Arbeit.

So wird auf operativer Prozessebene verstärkt auf leistungswirtschaftliche (Mengen-)Kennzahlen zurückgegriffen, wăhrend auf Top-Ebene vornehmlich finanzielle Größen betrachtet werden. Vgl. Euske/Lebas/McNair (1993), S. 287-289 sowie Schweitzer (2003), S. 440ff.

Im Rahmen der Datenerhebung wurden lediglich Banken, Versicherungen sowie sonstige Finanzdienstleister ausgeschlossen. Vgl. hierzu die Ausführungen in Fußnote 163 der vorliegenden Arbeit. 
wendete Rechnungslegungsstandard oder die Änderungsdynamik des Unternehmensumfeldes, einen moderierenden Einfluss auf die Dependenzbeziehungen des Kovarianzstrukturmodells haben.

Darüber hinaus wurden die der Analyse zu Grunde liegenden Daten vor Inkrafttreten des Bilanzrechtsmodernisierungsgesetzes (BilMoG) erhoben. ${ }^{481}$ Das BilMoG hat weitreichende Konsequenzen für die Bilanzierungs- und Bewertungsvorschriften des HGB, da es diese den Regelungen der IFRS annähert. ${ }^{482}$ Zukünftige empirische Studien könnten folglich untersuchen, welche Auswirkungen diese gesetzlichen Änderungen auf die Integrationsintensität des Rechnungswesens der nach HGB bilanzierenden Unternehmen haben. ${ }^{483}$

Der Einfluss einer Integration des Rechnungswesens auf die rollenbezogene Ausgestaltung sowie den Erfolg der Controllerarbeit wurde im vorliegenden Fall auf Basis einer Querschnittuntersuchung und somit in Form eines statischen Designs analysiert. Da Kausalitäten auf diese Weise aus wissenschaftstheoretischer Sicht streng genommen nicht überprüft werden können, ${ }^{484}$ bietet sich für die weitere Forschung die Durchführung einer Längsschnittuntersuchung an. Eine solche dynamische Betrachtung könnte aufschlussreiche Erkenntnisse hinsichtlich der Auswirkungen einer Änderung in der Integrationsintensität des Rechnungswesens auf die Controllerarbeit liefern.

Eine weitere Limitation liegt in der quasi-formativen Operationalisierung der Variablen 'Gesamtintegrationsintensität des Rechnungswesens' unter Zuhilfenahme eines Indexes. Da dieser Index als manifeste Variable in das Untersuchungsmodell eingeht, besitzt er keinen Fehlerterm, den formative latente Variablen normalerweise aufweisen. Dieser Fehlerterm repräsentiert

"the impact of all remaining causes other than those represented by the indicators included in the model. “485

Das BilMoG wurde am 28.5.2009 im Bundesgesetzblatt verkündet und ist damit am 29.5.2009 in Kraft getreten. In der Literatur wird diesbezüglich erwartet, dass die Änderungen des BilMoG die Integration des internen und externen Rechnungswesens fordern, da sie das Ziel einer verbesserten Informationsversorgung verfolgen, vgl. Barth/Kyank (2008), S. 846. 
Die Verwendung des Indexes unterstellt demnach implizit, dass die zu Grunde liegenden Indikatoren das Konstrukt vollständig erfassen, was in den meisten Fällen unzutreffend ist. ${ }^{486}$ Allerdings weist Diamantopoulos (2006) darauf hin, dass dieser Ansatz dann legitim ist, wenn alle möglichen Indikatoren eines Konstrukts spezifiziert werden können. ${ }^{487}$ Aufgrund der umfassenden Messung, die auf dem House of Controlling als konzeptionellem Bezugsrahmen basiert, dürfte diese Anforderung im vorliegenden Fall weitgehend erfültt sein. Dennoch sollte in zukünftigen empirischen Untersuchungen eine formative Operationalisierung - wie in Abschnitt E2.2 gezeigt wurde, war dies im Rahmen des vorliegenden Untersuchungsmodells nicht möglich - vorgenommen werden.

Zusätzlicher Forschungsbedarf ergibt sich darüber hinaus bezüglich der Einheitlichkeit der Finanzsprache, da mit einem $\mathrm{R}^{2}$-Wert von 0,18 nur ein vergleichsweise niedriger Varianzanteil dieser Variablen erklärt wird. ${ }^{488}$ Offensichtlich existieren neben der Integration des Rechnungswesens weitere bedeutsame Faktoren, die die Einheitlichkeit der Finanzsprache erklären und im vorliegenden Untersuchungsmodell nicht enthalten sind. Zur Identifizierung und Analyse dieser Treiber sind verstärkt interdisziplinäre Anstrengungen - etwa unter Berücksichtigung soziolinguistischer oder verhaltenswissenschaftlicher Ansätze, wie z.B. dem Konzept der mentalen Modelle ${ }^{489}$ - im Rahmen der weiteren Forschung erforderlich. Nach Denzau/North (1994) sind mentale Modelle

"the internal representations that individual cognitive systems create to interpret the environment" ${ }^{490}$

Der enge Zusammenhang von gemeinsamen mentalen Modellen, den so genannten 'Shared Mental Models' (SMM), und dem Aspekt einer einheitlichen Sprache bzw. verbesserten Kommunikation wird hier bereits deutlich:

"To the extent that the speaker and a listener have common features in their mental models for the concepts identified in the SMM, they are more likely to be

Vgl. Diamantopoulos/Riefler/Roth (2008), S. 1215.

Vgl. Diamantopoulos (2006), S. $11 \mathrm{f}$.

Für eine analoge Argumentation bei einem $\mathrm{R}^{2}$-Wert in Höhe von $0,159 \mathrm{vgl}$. Schäffer/Zyder (2007), S. 23.

Zum Konzept mentaler Modelle vgl. grundlegend Denzau/North (1994) sowie Weber/Grothe/ Schäffer (2000).

Denzau/North (1994), S. 4. 
able to encode and decode their internal ideas into a shared language, and more likely be able to effectively communicate using single terms to stand for substantial pieces of implicit analysis embodied in the SMM. “491

Gemeinsame mentale Modelle reduzieren die Komplexität und bereiten somit die Grundlage für die Entstehung kooperativer Strukturen, da sie die Kommunikation zwischen Akteuren durch eine Konvergenz der jeweiligen Wahrnehmungs-, Prognoseund Bewertungsprozesse erleichtern. ${ }^{492}$

Insofern könnte im Rahmen zukünftiger Forschungsanstrengungen durch den Rückgriff auf das Konzept gemeinsamer mentaler Modelle möglicherweise ein umfassenderes Verständnis darüber entwickelt werden, welche Faktoren dazu beitragen, dass die im Unternehmen vorgehaltenen Finanzinformationen als einheitlich und konsistent wahrgenommen werden. Wie die vorliegende Untersuchung gezeigt hat, liegt in der Einheitlichkeit der Finanzsprache ein wichtiger - und zugleich seitens der Controller im Vergleich zu den Managern unterschätzter - Erfolgsfaktor für die Controllerarbeit. Vor diesem Hintergrund ist Hirsch (2007) in seiner Einschätzung zuzustimmen, dass in einer Berücksichtigung

„verhaltenswissenschafticher Erkenntnisse in der (deutschen) Controllingforschung ein erhebliches Potential “493

liegt. Dieses gilt es auch bezogen auf das in dieser Arbeit adressierte Themenfeld weiter zu heben.

491 Denzau/North (1994), S. 20. Zum Zusammenhang mentaler Modelle, konzeptioneller Informationsnutzung und der Sichtweise des Rechnungswesens als Sprache vgl. auch Schaier (2007), S. 188 sowie Meyer (2007), S. 4. Zu alternativen Techniken der empirischen Messung gemeinsamer mentaler Modelle vgl. Mohammed/Klimoski/Rentsch (2000). 
Hendrik Angelkort - 978-3-631-75143-5

Downloaded from PubFactory at 01/11/2019 07:47:42AM

via free access 


\section{Literaturverzeichnis}

Anderson, James C./Gerbing, David W. (1984): The Effect of Sampling Error on Convergence, Improper Solutions, and Goodness-of-Fit Indices for Maximum Likelihood Confirmatory Factor Analysis, in: Psychometrika, Vol. 49, S. 155-173.

Anderson, James C./Gerbing, David W. (1988): Structural Equation Modeling in Practice: A Review and Recommended Two-Step Approach, in: Psychological Bulletin, Vol. 103, S. 411-423.

Angelkort, Hendrik/Sandt, Joachim/Weißenberger, Barbara E. (2008a): IFRSRechnungslegung - Risiko oder Erfolgsfaktor für die Controllerarbeit? - Proaktive Auseinandersetzung mit den IFRS als Erfolgsgrundlage, in: CFO aktuell - Zeitschrift für Finance \& Controlling, 2. Jg., S. 188-191.

Angelkort, Hendrik/Sandt, Joachim/Weißenberger, Barbara E. (2008b): Veränderungen der Controllership unter IFRS - Konzeptionelle Überlegungen und empirische Ergebnisse, in: Weber, Jürgen/Vater, Hendrik/Schmidt, Walter/Reinhard, Hartmut/Ernst, Edgar (Hrsg.): Die neue Rolle des Controllers, Stuttgart, S. 61-80.

Arbuckle, James L. (2007): Amos 16.0 User's Guide, Chicago.

Armstrong, J. Scott/Overton, Terry S. (1977): Estimating Nonresponse Bias in Mail Surveys, in: Journal of Marketing Research, Vol. 14, S. 396-402.

Arnold, Hugh J. (1982): Moderator Variables: A Clarification of Conceptual, Analytic and Psychometric Issues, in: Organizational Behavior and Human Performance, Vol. 29, S. 143-174.

Atteslander, Peter (2008): Methoden der empirischen Sozialforschung, 12. Aufl., Berlin.

Backhaus, Klaus/Blechschmidt, Boris (2009): Fehlende Werte und Datenqualität Eine Simulationsstudie am Beispiel der Kausalanalyse, in: Die Betriebswirtschaft, 69. Jg., S. 265-287. 
Backhaus, Klaus/Erichson, Bernd/Plinke, Wulff/Weiber, Rolf (2006): Multivariate Analysemethoden - Eine anwendungsorientierte Einführung, 11. Aufl., Berlin et al.

Backhaus, Klaus/Erichson, Bernd/Plinke, Wulff/Weiber, Rolf (2008): Multivariate Analysemethoden - Eine anwendungsorientierte Einführung, 12. Aufl., Berlin et al.

Bagozzi, Richard P. (1982): Evaluating Structural Equation Models with Unobservable Variables and Measurement Error: A Comment, in: Fornell, Claes (Hrsg.): A Second Generation of Multivariate Analysis, New York, S. 317-330.

Bagozzi, Richard P. (1994): Structural Equation Models in Marketing Research: Basic Principles, in: Bagozzi, Richard P. (Hrsg.): Principles of Marketing Research, Cambridge, S. 317-385.

Bagozzi, Richard P./Baumgartner, Hans (1994): The Evaluation of Structural Equation Models and Hypothesis Testing, in: Bagozzi, Richard P. (Hrsg.): Principles of Marketing Research, Cambridge, S. 386-422.

Bagozzi, Richard P./Fornell, Claes (1982): Theoretical Concepts, Measurements, and Meaning, in: Fornell, Claes (Hrsg.): A Second Generation of Multivariate Analysis, New York, S. 24-38.

Bagozzi, Richard P./Yi, Youjae (1988): On the Evaluation of Structural Equation Models, in: Journal of the Academy of Marketing Science, Vol. 16, S. 74-94.

Balderjahn, Ingo (2008): Editorial: Totgeglaubte leben länger! Auch in der BWL?, in: Die Betriebswirtschaft, 68. Jg., S. 267-269.

Baron, Reuben M./Kenny, David A. (1986): The Moderator-Mediator Variable Distinction in Social Psychological Research: Conceptual, Strategic, and Statistical Considerations, in: Journal of Personality and Social Psychology, Vol. 51, S. $1173-1182$.

Barth, Thomas/Kyank, Matthias (2008): Bilanzrechtsmodernisierungsgesetz und Controlling, in: Das Wirtschaftsstudium, 37. Jg., S. 841-846. 
Bauer, Michael (2002): Controllership in Deutschland - Zur erfolgreichen Zusammenarbeit von Controllern und Managern, Wiesbaden.

Bearden, William O./Netemeyer, Richard G./Teel, Jesse E. (1989): Measurement of Consumer Susceptibility to Interpersonal Influence, in: Journal of Consumer Research, Vol. 15, S. 473-481.

Becker, Wolfgang/Baltzer, Björn (2009a): Die Struktur des betrieblichen Rechnungswesens unter Berücksichtigung aktueller Entwicklungen - Teil 1, in: Wirtschaftswissenschaftliches Studium, 38. Jg., S. 173-179.

Becker, Wolfgang/Baltzer, Björn (2009b): Die Struktur des betrieblichen Rechnungswesens unter Berücksichtigung aktueller Entwicklungen - Teil 2, in: Wirtschaftswissenschaftliches Studium, 38. Jg., S. 226-231.

Beißel, Jörg/Steinke, Karl-Heinz (2004): Integriertes Reporting unter IFRS bei der Lufthansa, in: Zeitschrift für Controlling \& Management, Sonderheft 2/2004, S. 63-70.

Beißel, Jörg/Szczesny, Andrea (2009): Anreiznützlichkeit in einem konvergenten Berichtswesen - Grundsätzliche Gestaltungsregeln und deren Umsetzung bei der Deutschen Lufthansa AG, in: Controlling, 21. Jg., S. 23-28.

Belkaoui, Ahmed (1980): The Interprofesssional Linguistic Communication of Accounting Concepts: An Experiment in Sociolinguistics, in: Journal of Accounting Research, Vol. 18, S. 362-374.

Belkaoui, Ahmed (1989): Accounting and Language, in: Journal of Accounting Literature, Vol. 8, S. 281-292.

Bentler, Peter M. (1990): Comparative Fit Indexes in Structural Models, in: Psychological Bulletin, Vol. 107, S. 238-246.

Bentler, Peter M. (1995): EQS Structural Equations Program Manual, Encino. 
Blase, Steffen/Müller, Stefan (2009): Empirische Analyse der vorzeitigen IFRS-8Erstanwendung - Eine Analyse der Harmonisierung von interner und externer Segmentberichterstattung im Rahmen der vorzeitigen Umstellung auf IFRS 8 bei DAX-, MDAX- und SDAX-Unternehmen, in: Die Wirtschaftsprüfung, 62. Jg., S. 537-544.

Bloomfield, Robert J. (2008): Accounting as the Language of Business, in: Accounting Horizons, Vol. 22, S. 433-436.

Blunch, Niels J. (2008): Introduction to Structural Equation Modeling Using SPSS and AMOS, Los Angeles et al.

Boland, Richard J. Jr./Pondy, Louis R. (1983): Accounting in Organizations: A Union of Natural and Rational Perspectives, in: Accounting, Organizations and Society, Vol. 8, S. 223-234.

Bollen, Kenneth A. (1989): Structural Equations with Latent Variables, New York et al.

Bollen, Kenneth/Lennox, Richard (1991): Conventional Wisdom on Measurement: A Structural Equation Perspective, in: Psychological Bulletin, Vol. 110, S. 305314.

Bortz, Jürgen (2005): Statistik für Human- und Sozialwissenschaftler, 6. Aufl., Heidelberg.

Bortz, Jürgen/Döring, Nicola (2006): Forschungsmethoden und Evaluation für Human- und Sozialwissenschaftler, 4. Aufl., Heidelberg.

Bramann, Annehild (2009): Die Auswirkungen der Harmonisierung internationaler Rechnungslegung auf das Controlling von Forschung und Entwicklung, Frankfurt am Main.

Browne, Michael W./Cudeck, Robert (1993): Alternative Ways of Assessing Model Fit, in: Bollen, Kenneth/Long, J. Scott (Hrsg.): Testing Structural Equation Models, Newbury Park, S. 136-162. 
Brühl, Rolf (2008): Begriffe und Variable in der betriebswirtschaftlichen Theorienentwicklung, in: Wirtschaftswissenschaftliches Studium, 37. Jg., S. 363-368.

Bruns, Hans-Georg (1999): Harmonisierung des externen und internen Rechnungswesens auf Basis internationaler Bilanzierungsvorschriften, in: Küting, Karlheinz/Langenbucher, Günther (Hrsg.): Internationale Rechnungslegung - Festschrift für Professor Claus-Peter Weber zum 60. Geburtstag, Stuttgart, S. 585-603.

Bühl, Achim (2006): SPSS 14 - Einführung in die moderne Datenanalyse, 10. Aufl., München et al.

Buhleier, Claus (2008): Der IFRS Goodwill Impairment Test - Schnittpunkt zwischen Internationaler Rechnungslegung und Internationalem Controlling, in: Funk, Wilfried/Rossmanith, Jonas (Hrsg.): Internationale Rechnungslegung und Internationales Controlling, Wiesbaden, S. 455-479.

Bühner, Markus (2006): Einführung in die Test- und Fragebogenkonstruktion, 2. Aufl., München et al.

Byrne, Barbara M. (1989): A primer of LISREL: Basic Applications and Programming for Confirmatory Factor Analytic Models, New York.

Byrne, Barbara M. (2001): Structural Equation Modeling With AMOS - Basic Concepts, Applications and Programming, Mahwah/New Jersey.

Chenhall, Robert H. (2003): Management Control Systems Design within its Organizational Context: Findings from Contingency-based Research and Directions for the Future, in: Accounting, Organizations and Society, Vol. 28, S. 127-168.

Cheung, Gordon W./Lau, Rebecca S. (2008): Testing Mediation and Suppression Effects of Latent Variables - Bootstrapping With Structural Equation Models, in: Organizational Research Methods, Vol. 11, S. 296-325.

Chmielewicz, Klaus (1982): Betriebliches Rechnungswesen 1 - Finanzrechnung und Bilanz, 3. Aufl., Opladen.

Chmielewicz, Klaus (1994): Forschungskonzeptionen der Wirtschaftswissenschaft, 3. Aufl., Stuttgart. 
Christophersen, Timo/Grape, Christian (2007): Die Erfassung latenter Konstrukte mithilfe formativer und reflektiver Messmodelle, in: Albers, Sönke/Klapper, Daniel/Konradt, Udo/Walter, Achim/Wolf, Joachim (Hrsg.): Methodik der empirischen Forschung, 2. Aufl., Wiesbaden, S. 103-118.

Churchill, Gilbert A., Jr. (1979): A Paradigm for Developing Better Measures of Marketing Constructs, in: Journal of Marketing Research, Vol. 16, S. 64-73.

Coenenberg, Adolf G. (1995): Einheitlichkeit oder Differenzierung von internem und externem Rechnungswesen: Die Anforderungen der internen Steuerung, in: Der Betrieb, 48. Jg., S. 2077-2083.

Cronbach, Lee J. (1951): Coefficient Alpha and the Internal Structure of Tests, in: Psychometrika, Vol. 16, S. 297-334.

Dais, Martin/Watterott, Richard (2006): Umstellung des externen und internen Rechnungswesens der Bosch-Gruppe auf IFRS - Harmonisierung und deren Grenzen, in: Controlling, 18. Jg., S. 465-473.

Daum, Jürgen H. (2008): Die Entwicklung der Rolle des CFO in europäischen Unternehmen - Auswirkungen auf und Zukunftsperspektiven für Controller und Controlling - Ein Praxisbericht, in: Zeitschrift für Controlling \& Management, 52. Jg., S. 387-393.

Denzau, Arthur T./North, Douglass C. (1994): Shared Mental Models: Ideologies and Institutions, in: Kyklos, Vol. 47, S. 3-31.

Diamantopoulos, Adamantios (2006): The Error Term in Formative Measurement Models: Interpretation and Modeling Implications, in: Journal of Modelling in Management, Vol. 1, S. 7-17.

Diamantopoulos, Adamantios (2008): Formative Indicators: Introduction to the Special Issue, in: Journal of Business Research, Vol. 61, S. 1201-1202.

Diamantopoulos, Adamantios/Riefler, Petra/Roth, Katharina P. (2008): Advancing Formative Measurement Models, in: Journal of Business Research, Vol. 61, S. 1203-1218. 
Diamantopoulos, Adamantios/Winklhofer, Heidi M. (2001): Index Construction with Formative Indicators: An Alternative to Scale Development, in: Journal of Marketing Research, Vol. 38, S. 269-277.

Dörr, Barbara (2004): Fast Close - Geschwindigkeit und Transparenz im Abschluss, in: Küting, Karlheinz/Pfitzer, Norbert/Weber, Claus-Peter (Hrsg.): Herausforderungen und Chancen durch weltweite Rechnungslegungsstandards, Stuttgart, S. 403-428.

Eberl, Markus (2004): Formative und reflektive Indikatoren im Forschungsprozess: Entscheidungsregeln und die Dominanz des reflektiven Modells, Schriften zur Empirischen Forschung und Quantitativen Unternehmensplanung, Heft 19/2004, München.

Eberl, Markus (2006): Formative und reflektive Konstrukte und die Wahl des Strukturgleichungsverfahrens - Eine statistische Entscheidungshilfe, in: Die Betriebswirtschaft, 66. Jg., S. 651-668.

Efron, Bradley (1987): Better Bootstrap Confidence Intervals, in: Journal of the American Statistical Association, Vol. 82, S. 171-185.

Eggemann, Gerd/Petry, Martin (2002): Fast Close - Verkürzung von Aufstellungsund Veröffentlichungszeiten für Jahres- und Konzernabschlüsse, in: BetriebsBerater, 57. Jg., S. 1635-1638.

Eierle, Brigitte/Schultze, Wolfgang/Bischof, Bettina/Thiericke, Sandra (2008): Eignung der IFRS für Controllingzwecke - Ergebnisse einer empirischen Befragung nicht kapitalmarktorientierter Unternehmen, in: Controlling, 20. Jg., S. 289298.

Eisele, Wolfgang (2002): Technik des betrieblichen Rechnungswesens - Buchführung und Bilanzierung, Kosten- und Leistungsrechnung, Sonderbilanzen, 7. Aufl., München. 
Erdmann, Mark-Ken (2008): Die Akzeptanz der IFRS als Grundlage der wertorientierten Unternehmenssteuerung basiert auf der Frage, ob die IFRS Regelungen wirtschaftlich nachvollziehbar sind, in: Zeitschrift für Controlling \& Management, 52. Jg., S. 225-227.

Erichsen, Jörgen (2000): Zusammenführung von externem und internem Rechnungswesen - Verbesserung der Effizienz des Controllings, in: bilanz \& buchhaltung, 46. Jg., S. 55-59.

Ernst, Edgar/Poschmann, Sarah/Reinhard, Hartmut/Vater, Hendrik (2008): Entwicklung der Rolle des Controllers - Eigen- versus Fremdbild, in: Weber, Jürgen/Vater, Hendrik/Schmidt, Walter/Reinhard, Hartmut/Ernst, Edgar (Hrsg.): Die neue Rolle des Controllers, Stuttgart, S. 47-59.

Ernst, Holger (2003): Ursachen eines Informant Bias und dessen Auswirkung auf die Validität empirischer betriebswirtschaftlicher Forschung, in: Zeitschrift für Betriebswirtschaft, 73. Jg., S. 1249-1275.

Esser, Joachim/Müller, Michael (2007): Empirische Erkenntnisse zur Organisation des Controllings, in: Gleich, Ronald/Michel, Uwe (Hrsg.): Organisation des Controllings - Grundlagen, Praxisbeispiele und Perspektiven, Freiburg et al., S. 33-54.

Europäische Union (2003): Empfehlung der Kommission vom 6. Mai 2003 betreffend die Definition der Kleinstunternehmen sowie der kleinen und mittleren Unternehmen, Amtsblatt der Europäischen Union Nr. L 124 vom 20.5.2003 - abrufbar unter http://eur-lex.europa.eu/LexUriServ/LexUriServ.do?uri=OJ:L:2003:124: 0036:0041:DE:PDF (Datum des letzten Abrufs: 14.10.2009).

Euske, K. J./Lebas, M. J./McNair, C. J. (1993): Performance Management in an International Setting, in: Management Accounting Research, Vol. 4, S. 275-299.

Evans, Lisa (2004): Language, Translation and the Problem of International Accounting Communication, in: Accounting, Auditing \& Accountability Journal, Vol. 17, S. 210-248. 
Ewert, Ralf (2006): Fair Value-Bewertung und Performancemessung, in: Börsig, Clemens/Wagenhofer, Alfred (Hrsg.): IFRS in Rechnungswesen und Controlling Kongress-Dokumentation, 59. Deutscher Betriebswirtschafter-Tag 2005, Stuttgart, S. 179-207.

Ewert, Ralf/Wagenhofer, Alfred (2008): Interne Unternehmensrechnung, 7. Aufl., Berlin et al.

Fassott, Georg (2006): Operationalisierung latenter Variablen in Strukturgleichungsmodellen: Eine Standortbestimmung, in: Zeitschrift für betriebswirtschaftliche Forschung, 58. Jg., S. 67-88.

Fassott, Georg/Eggert, Andreas (2005): Zur Verwendung formativer und reflektiver Indikatoren in Strukturgleichungsmodellen: Bestandsaufnahme und Anwendungsempfehlungen, in: Bliemel, Friedhelm/Eggert, Andreas/Fassott, Georg/Henseler, Jörg (Hrsg.): Handbuch PLS-Pfadmodellierung - Methode, Anwendung, Praxisbeispiele, Stuttgart, S. 31-47.

Festinger, Leon (1957): A Theory of Cognitive Dissonance, Stanford.

Fleischer, Werner (2005): Rolle des Controllings im Spannungsfeld internes und externes Reporting, in: Horváth, Péter (Hrsg.): Organisationsstrukturen und Geschäftsprozesse wirkungsvoll steuern, Stuttgart, S. 189-200.

Fornell, Claes/Larcker, David F. (1981): Evaluating Structural Equation Models with Unobservable Variables and Measurement Error, in: Journal of Marketing Research, Vol. 18, S. 39-50.

Fox, John (1980): Effect Analysis in Structural Equation Models - Extensions and Simplified Methods of Computation, in: Sociological Methods \& Research, Vol. 9, S. 3-28.

Frane, James W. (1976): Some Simple Procedures for Handling Missing Data in Multivariate Analysis, in: Psychometrika, Vol. 41, S. 409-415.

Franz, Klaus-Peter/Winkler, Carsten (2006): Unternehmenssteuerung und IFRS Grundlagen und Praxisbeispiele, München. 
Goetzke, Wolfgang (1979): Aufgabenanalyse als Grundlage der Gestaltung des Rechnungswesens nicht erwerbswirtschaftlicher Betriebe, in: Betriebswirtschaftliche Forschung und Praxis, 31. Jg., S. 517-535.

Gregson, Terry (1992): The Advantages of LISREL for Accounting Researchers, in: Accounting Horizons, Vol. 6, S. 42-48.

Greving, Bert (2007): Messen und Skalieren von Sachverhalten, in: Albers, Sönke/Klapper, Daniel/Konradt, Udo/Walter, Achim/Wolf, Joachim (Hrsg.): Methodik der empirischen Forschung, 2. Aufl., Wiesbaden, S. 65-78.

Grieshop, Hendrik/Weber, Jürgen (2007): Ergebnisse einer empirischen Studie zur Kooperation von Controllerbereich und externem Rechnungswesen, in: Der Konzern, 5. Jg., S. 307-315.

Grochla, Erwin (1976): Praxeologische Organisationstheorie durch sachliche und methodische Integration - Eine pragmatische Konzeption, in: Zeitschrift für betriebswirtschaftliche Forschung, 28. Jg., S. 617-637.

Günther, Thomas/Zurwehme, Annikka (2008): Harmonisierung des Rechnungswesens - Stellschrauben, Informationswirkung und Nutzenbewertung, in: Betriebswirtschaftliche Forschung und Praxis, 60. Jg., S. 101-121.

Haas, Cornelia A. J. (2009): Goodwill-Bilanzierung nach IFRS und Implikationen für das Controlling, Lohmar/Köln.

Haeger, Bernd (2006): Harmonisierung von Rechnungswesen und Controlling bei E.ON, in: Wagenhofer, Alfred (Hrsg.): Controlling und IFRS-Rechnungslegung Konzepte, Schnittstellen, Umsetzung, Berlin, S. 243-266.

Hahn, Dietger/Hungenberg, Harald (2001): PuK - Planung und Kontrolle, Planungs- und Kontrollsysteme, Planungs- und Kontrollrechnung, 6. Aufl., Wiesbaden.

Haller, Axel (1997a): Herausforderungen an das Controlling durch die Internationalisierung der externen Rechnungslegung, in: Horváth, Peter (Hrsg.): Das neue Steuerungssystem des Controllers - Von Balanced Scorecard bis US-GAAP, Stuttgart, S. 113-131. 
Haller, Axel (1997b): Zur Eignung der US-GAAP für Zwecke des internen Rechnungswesens, in: Controlling, 9. Jg., S. 270-276.

Haller, Axel (2006): Segmentberichterstattung - Schnittstelle zwischen Controlling und Rechnungslegung, in: Wagenhofer, Alfred (Hrsg.): Controlling und IFRSRechnungslegung - Konzepte, Schnittstellen, Umsetzung, Berlin, S. 143-168.

Haring, Nikolai/Prantner, Renate (2005): Konvergenz des Rechnungswesens. Stateof-the-Art in Deutschland und Österreich, in: Controlling, 17. Jg., S. 147-154.

Hauser, Robert M./Goldberger, Arthur S. (1971): The Treatment of Unobservable Variables in Path Analysis, in: Sociological Methodology, Vol. 3, S. 81-117.

Havermann, Hans (2000): Konzernrechnungslegung - quo vadis?, in: Die Wirtschaftsprüfung, 53. Jg., S. 121-127.

Hayes, David C. (1983): Accounting for Accounting: A Story about Managerial Accounting, in: Accounting, Organizations and Society, Vol. 8, S. 241-249.

Hebeler, Christian (2003): Harmonisierung des internen und externen Rechnungswesens - US-amerikanische Accounting-Systeme als konzeptionelle Grundlage für deutsche Unternehmen?, Wiesbaden.

Hebeler, Christian (2006): Harmonisierung des internen und externen Rechnungswesens bei Henkel, in: Horváth, Péter (Hrsg.): Controlling und Finance Excellence, Stuttgart, S. 111-127.

Heine, Bernd-Oliver (2008): Konzeptionelle Nutzung von Controllinginformationen - Ein modelltheoretischer Ansatz, Wiesbaden.

Herrmann, Andreas/Huber, Frank/Kressmann, Frank (2006): Varianz- und kovarianzbasierte Strukturgleichungsmodelle - Ein Leitfaden zu deren Spezifikation, Schätzung und Beurteilung, in: Zeitschrift für betriebswirtschaftliche Forschung, 58. Jg., S. 34-66.

Heyd, Reinhard (2001): Zur Harmonisierung von internem und externem Rechnungswesen nach US-GAAP, in: Der Schweizer Treuhänder, 75. Jg., S. 201-214. 
Hildebrandt, Lutz (1984): Kausalanalytische Valdierung in der Marketingforschung, in: Marketing: Zeitschrift für Forschung und Praxis, 6. Jg., S. 41-51.

Hirsch, Bernhard (2007): Controlling und Entscheidungen - Zur verhaltenswissenschaftlichen Fundierung des Controllings, Tübingen.

Hoffjan, Andreas (2008): Comparative Management Accounting - Vergleich des anglo-amerikanischen Management Accounting und des deutschen Controllings, in: Controlling, 20. Jg., S. 655-661.

Hoke, Michaela (2001): Konzernsteuerung auf Basis eines intern und extern vereinheitlichten Rechnungswesens - Empirische Befunde vor dem Hintergrund der Internationalisierung der Rechnungslegung, Bamberg.

Homburg, Christian (1989): Exploratorische Ansätze der Kausalanalyse als Instrument der Marketingplanung, Frankfurt am Main et al.

Homburg, Christian (1992a): Die Kausalanalyse - Eine Einführung, in: Wirtschaftswissenschaftliches Studium, 21. Jg., S. 499-508.

Homburg, Christian (1992b): Beispiele zur Kausalanalyse, in: Wirtschaftswissenschaftliches Studium, 21. Jg., S. 541-544.

Homburg, Christian (2007): Betriebswirtschaftslehre als empirische Wissenschaft Bestandsaufnahme und Empfehlungen, in: Zeitschrift für betriebswirtschaftliche Forschung, Sonderheft 56/07, S. 27-60.

Homburg, Christian/Baumgartner, Hans (1995a): Beurteilung von Kausalmodellen - Bestandsaufnahme und Anwendungsempfehlungen, in: Marketing: Zeitschrift für Forschung und Praxis, 17. Jg., S. 162-176.

Homburg, Christian/Baumgartner, Hans (1995b): Die Kausalanalyse als Instrument der Marketingforschung - Eine Bestandsaufnahme, in: Zeitschrift für Betriebswirtschaft, 65. Jg., S. 1091-1108. 
Homburg, Christian/Dobratz, Andreas (1998): Iterative Modellselektion in der Kausalanalyse, in: Hildebrandt, Lutz/Homburg, Christian (Hrsg.): Die Kausalanalyse - Ein Instrument der empirischen betriebswirtschaftlichen Forschung, Stuttgart, S. 447-474.

Homburg, Christian/Giering, Annette (1996): Konzeptualisierung und Operationalisierung komplexer Konstrukte - Ein Leitfaden für die Marketingforschung, in: Marketing: Zeitschrift für Forschung und Praxis, 18. Jg., S. 5-24.

Homburg, Christian/Hildebrandt, Lutz (1998): Die Kausalanalyse: Bestandsaufnahme, Entwicklungsrichtungen, Problemfelder, in: Hildebrandt, Lutz/Homburg, Christian (Hrsg.): Die Kausalanalyse - Ein Instrument der empirischen betriebswirtschaftlichen Forschung, Stuttgart, S. 15-43.

Homburg, Christian/Klarmann, Martin (2006): Die Kausalanalyse in der empirischen betriebswirtschaftlichen Forschung - Problemfelder und Anwendungsempfehlungen, in: Die Betriebswirtschaft, 66. Jg., S. 727-748.

Homburg, Christian/Klarmann, Martin (2009): Multi Informant-Designs in der empirischen betriebswirtschaftlichen Forschung - Problemfelder und Anwendungsempfehlungen, in: Die Betriebswirtschaft, 69. Jg., S. 147-171.

Homburg, Christian/Pflesser, Christian (2000a): Konfirmatorische Faktorenanalyse, in: Herrmann, Andreas/Homburg, Christian (Hrsg.): Marktforschung - Methoden, Anwendungen, Praxisbeispiele, 2. Aufl., Wiesbaden, S. 413-437.

Homburg, Christian/Pflesser, Christian (2000b): Strukturgleichungsmodelle mit latenten Variablen: Kausalanalyse, in: Herrmann, Andreas/Homburg, Christian (Hrsg.): Marktforschung - Methoden, Anwendungen, Praxisbeispiele, 2. Aufl., Wiesbaden, S. 633-659.

Homburg, Christian/Stock, Ruth (2001): Der Zusammenhang zwischen Mitarbeiterund Kundenzufriedenheit: Eine dyadische Analyse, in: Zeitschrift für Betriebswirtschaft, 71. Jg., S. 789-806.

Horváth, Péter (2009): Controlling, 11. Aufl., München. 
Horváth, Péter/Arnaout, Ali (1997): Internationale Rechnungslegung und Einheit des Rechnungswesens - State-of-the-Art und Implementierung in der deutschen Praxis, in: Controlling, 9. Jg., S. 254-269.

Horváth, Péter/Pötsch, Hans Dieter (2008): Fragen zu Stand und Entwicklungstrends im Controlling, in: Controlling, 20. Jg., S. 707-709.

Huber, Frank/Heitmann, Mark/Herrmann, Andreas (2006): Ansätze zur Kausalmodellierung mit Interaktionseffekten - Ein Überblick, in: Die Betriebswirtschaft, 66. Jg., S. 696-710.

Hurrle, Beatrice/Kieser, Alfred (2005): Sind Key Informants verlässliche Datenlieferanten?, in: Die Betriebswirtschaft, 65. Jg., S. 584-602.

Hüttner, Manfred/Schwarting, Ulf (2000): Exploratorische Faktorenanalyse, in: Herrmann, Andreas/Homburg, Christian (Hrsg.): Marktforschung - Methoden, Anwendungen, Praxisbeispiele, 2. Aufl., Wiesbaden, S. 381-412.

Iacobucci, Dawn/Saldanha, Neela/Deng, Xiaoyan (2007): A Meditation on Mediation: Evidence That Structural Equations Models Perform Better Than Regressions, in: Journal of Consumer Psychology, Vol. 17, S. 139-153.

Illetschko, Leopold L. (1955): Dokumentar- und Instrumentalcharakter des betrieblichen Rechnungswesens, in: Illetschko, Leopold L. (Hrsg.): Management und Betriebswirtschaft - Vorträge und Aufsätze, Wien, S. 25-35.

International Group of Controlling (2009): Controllerleitbild - abrufbar unter http://www.igc-controlling.org/DE/_leitbild/leitbild.php (Datum des letzten Abrufs: 14.10 .2009$)$.

International Group of Controlling (Hrsg.) (2005): Controller-Wörterbuch, 3. Aufl., Stuttgart.

International Group of Controlling (Hrsg.)/Weißenberger, Barbara E. (2006): Controller und IFRS - Konsequenzen für die Controlleraufgaben durch die Finanzberichterstattung nach IFRS, Freiburg et al. 
Jahn, Steffen (2007): Strukturgleichungsmodellierung mit LISREL, AMOS und SmartPLS - Eine Einführung, Diskussionspapier 86/2007 der Fakultät für Wirtschaftswissenschaften der Technischen Universität Chemnitz.

Jahnke, Hermann/Wielenberg, Stefan/Schumacher, Heinrich (2007): Ist die Integration des Rechnungswesens tatsächlich ein Motiv für die Einführung der IFRS in mittelständischen Unternehmen?, in: Zeitschrift für internationale und kapitalmarktorientierte Rechnungslegung, 7. Jg., S. 365-376.

Janssen, Jürgen/Laatz, Wilfried (2007): Statistische Datenanalyse mit SPSS für Windows, 6. Aufl., Berlin et al.

Janusiak, Christof/Wittkowski, Marc (2008): State-of-the-Art der Konvergenz des Rechnungswesens in Theorie und Praxis von 1994 - 2007, unveröffentlichte Diplomarbeit an der Professur für Controlling und integrierte Rechnungslegung, Justus-Liebig-Universität Gießen.

Jarvis, Cheryl Burke/Mackenzie, Scott B./Podsakoff, Philip M. (2003): A Critical Review of Construct Indicators and Measurement Model Misspecification in Marketing and Consumer Research, in: Journal of Consumer Research, Vol. 30, S. 199-218.

Jonen, Andreas/Lingnau, Volker (2005): Internes und externes Rechnungswesen Theoretische Überlegungen zur Konvergenz und Vorstellung der praktischen Umsetzung, in: Seicht, Gerhard (Hrsg.): Jahrbuch für Controlling und Rechnungswesen 2005, Wien, S. 281-315.

Jöreskog, Karl G. (1966): Testing a Simple Structure Hypothesis in Factor Analysis, in: Psychometrika, Vol. 31, S. 165-178.

Jöreskog, Karl G. (1967): Some Contributions to Maximum Likelihood Factor Analysis, in: Psychometrika, Vol. 32, S. 443-482.

Jöreskog, Karl G. (1969): A General Approach to Confirmatory Maximum Likelihood Factor Analysis, in: Psychometrika, Vol. 34, S. 183-202.

Jöreskog, Karl G./Sörbom, Dag (1981): LISREL V - Analysis of Linear Structural Relationships by Maximum Likelihood and Least Squares Methods, Chicago. 
Jöreskog, Karl G./Sörbom, Dag (1982): Recent Developments in Structural Equation Modeling, in: Journal of Marketing Research, Vol. 19, S. 404-416.

Kaiser, Henry F. (1974): An Index of Factorial Simplicity, in: Psychometrika, Vol. 39, S. 31-36.

Kajüter, Peter (2008): Harmonisierung des Rechnungswesens: Ein Ansatz zur Vereinfachung des Controllings?, in: vom Brocke, Jan/Becker, Jörg (Hrsg.): Einfachheit in Wirtschaftsinformatik und Controlling, S. 337-354.

Karl, Stefan (2006): Optimierte Integration des externen und internen Rechnungswesens mit mySAP ERP, in: Controlling, 18. Jg., S. 455-463.

Kenny, David A./Judd, Charles M. (1984): Estimating the Nonlinear and Interactive Effects of Latent Variables, in: Psychological Bulletin, Vol. 96, S. 201-210.

Kirsch, Hans-Jürgen/Ewelt, Corinna (2008): Die Konvergenz von internem und externem Rechnungswesen: Vereinfachung und Qualitätssteigerung durch Vereinheitlichung, in: vom Brocke, Jan/Becker, Jörg (Hrsg.): Einfachheit in Wirtschaftsinformatik und Controlling, S. 307-320.

Klein, Georg A. (1999): Unternehmenssteuerung auf Basis der International Accounting Standards - Ein Beitrag zur Konvergenz von internem und externem Rechnungswesen, München.

Kley, Karl-Ludwig (2006): IFRS - Möglichkeiten und Grenzen ihrer Abbildung im Controlling, in: Zeitschrift für Controlling \& Management, 50. Jg., S. 150-157.

Knobel, Carsten/Vordermark, Uwe (2008): Die Rolle des Controllers bei Henkel, in: Weber, Jürgen/Vater, Hendrik/Schmidt, Walter/Reinhard, Hartmut/Ernst, Edgar (Hrsg.): Die neue Rolle des Controllers, Stuttgart, S. 183-194.

Knollmann, Ramon (2007): Kooperation von Controllerbereich und Strategieabteilung - Messung, Wirkungen, Determinanten, Wiesbaden.

Krey, Sandra (2007): Integration von legalem und Management-Berichtswesen auf eine Systemplattform - dargestellt am Beispiel der Einführung von SAP SEMBCS bei MAN, in: Controlling, 19. Jg., S. 231-236. 
Kumar, Nirmalya/Stern, Louis W./Anderson, James C. (1993): Conducting Interorganizational Research Using Key Informants, in: Academy of Management Journal, Vol. 36, S. 1633-1651.

Kümmel, Gerhard/Watterott, Richard (2005): Neue Entwicklungen im internationalen Konzerncontrolling am Beispiel Bosch - Durchgängige Konzernsteuerung, in: Horváth, Péter (Hrsg.): Organisationsstrukturen und Geschäftsprozesse wirkungsvoll steuern, Stuttgart, S. 11-32.

Kümpel, Thomas (2002): Vereinheitlichung von internem und externem Rechnungswesen, in: Wirtschaftswissenschaftliches Studium, 31. Jg., S. 343-345.

Künkele, Julia/Schäffer, Utz (2007): Zur erfolgreichen Gestaltung der Budgetkontrolle - Ergebnisse einer empirischen Untersuchung, in: Die Betriebswirtschaft, 67. Jg., S. 75-92.

Küpper, Hans-Ulrich (1998): Angleichung des externen und internen Rechnungswesens, in: Börsig, Clemens/Coenenberg, Adolf G. (Hrsg.): Controlling und Rechnungswesen im internationalen Wettbewerb - Kongress-Dokumentation, 51. Deutscher Betriebswirtschafter-Tag 1997, Stuttgart, S. 143-162.

Küpper, Hans-Ulrich (2008): Controlling - Konzeption, Aufgaben, Instrumente, 5. Aufl., Stuttgart.

Küting, Karlheinz/Lorson, Peter (1998): Konvergenz von internem und externem Rechnungswesen: Anmerkungen zu Strategien und Konfliktfeldern, in: Die Wirtschaftsprüfung, 51. Jg., S. 483-493.

Küting, Karlheinz/Lorson, Peter (1999): Harmonisierung des Rechnungswesens aus Sicht der externen Rechnungslegung, in: Kostenrechnungspraxis, Sonderheft 3/99, S. 47-57.

Landis, Ronald S./Beal, Daniel J./Tesluk, Paul E. (2000): A Comparison of Approaches to Forming Composite Measures in Structural Equation Models, in: Organizational Research Methods, Vol. 3, S. 186-207. 
Langthaler, Silvia (2008): Interview mit Siegfried Gänßlen zu dem Thema „Business Partnering: Mitarbeiter fordern und fördern“, in: Controller Magazin, 33. Jg., S. 12-14.

Lavoie, Don (1987): The Accounting of Interpretations and the Interpretation of Accounts: The Communicative Function of 'The Language of Business', in: Accounting, Organizations and Society, Vol. 12, S. 579-604.

Liedl, Reinhard (2005): Wandel in der Controllership, in: Zeitschrift für Controlling \& Management, 49. Jg., S. 183-184.

Likert, Rensis (1932): A Technique for the Measurement of Attitudes, in: Archives of Psychology, Vol. 22, S. 1-55.

Linder, Stefan/Wolf, Sebastian (2008): CFO und Controllerorganisation - Aktuelle Herausforderungen und Implikationen, in: CFO aktuell - Zeitschrift für Finance \& Controlling, 2. Jg., S. 160-164.

Lutz, Richard (2007): Effizienz und Wirkungsgrad im Controlling - Herausforderungen und Praxiskonzepte im Deutsche Bahn-Konzern, in: Horváth, Peter (Hrsg.): Erfolgstreiber für das Controlling, Stuttgart, S. 99-109.

MacCallum, Robert C./Browne, Michael W. (1993): The Use of Causal Indicators in Covariance Structure Models: Some Practical Issues, in: Psychological Bulletin, Vol. 114, S. 533-541.

Maier, Michael Thomas (2009): Der Management Approach - Herausforderungen für Controller und Abschlussprüfer im Kontext der IFRS-Finanzberichterstattung, Frankfurt am Main et al.

Marsh, Herbert W./Wen, Zhonglin/Hau, Kit-Tai (2006): Structural Equation Models of Latent Interaction and Quadratic Effects, in: Hancock, Gregory R./Mueller, Ralph O. (Hrsg.): Structural Equation Modeling - A Second Course, Greenwich, S. 225-265.

Mayer, Horst Otto (2009): Interview und schriftliche Befragung - Entwicklung, Durchführung und Auswertung, 5. Aufl., München/Wien. 
Melcher, Winfried (2002): Konvergenz von internem und externem Rechnungswesen - Umstellung des traditionellen Rechnungswesens und Einführung eines abgestimmten vertikalen und horizontalen Erfolgsspaltungskonzepts, Hamburg.

Menon, Anin/Varadarajan, P. Rajan (1992): A Model of Marketing Knowledge Use Within Firms, in: Journal of Marketing, Vol. 56, S. 53-71.

Messner, Stephanie (2003): Die Kostenrechnung im Spannungsfeld internationaler Entwicklungen - Über die Amerikanisierung des Rechnungswesens und ihre Bedeutung für die interne Unternehmensrechnung in Österreich und Deutschland, Wien.

Meyer, Matthias (2007): Integration von Kognition und Anreizen in die Rechnungswesenforschung, Vallendar.

Michel, Uwe (2006): Der Finanzbereich im Umbruch, in: Controlling, 18. Jg., S. 439445.

Michel, Uwe (2007): Shared Services als Organisationsform für das Controlling, in: Gleich, Ronald/Michel, Uwe (Hrsg.): Organisation des Controllings - Grundlagen, Praxisbeispiele und Perspektiven, Freiburg et al., S. 269-294.

Mohammed, Susan/Klimoski, Richard/Rentsch, Joan R. (2000): The Measurement of Team Mental Models: We Have No Shared Schema, in: Organizational Research Methods, Vol. 3, S. 123-165.

Moussallem, Steffen (2005): Optimierung des internen Berichtswesens, in: Accounting, 5. Jg., Heft 9, S. 11.

Moussallem, Steffen (2006): Veränderungen der Inhalte, Strukturen und Funktionen des Controllings unter IFRS, in: Accounting, 6. Jg., Heft 4, S. 12-15.

Müller, Martin (2006): Harmonisierung des externen und internen Rechnungswesens - Eine empirische Untersuchung, Wiesbaden.

Nunnally, Jum C. (1978): Psychometric Theory, 2. Aufl., New York et al. 
Otley, D. T., Berry, A.J. (1980): Control, Organisation, and Accounting, in: Accounting, Organizations and Society, Vol. 5, S. 231-244.

Pellens, Bernhard/Fülbier, Rolf Uwe/Gassen, Joachim/Sellhorn, Thorsten (2008): Internationale Rechnungslegung, 7. Aufl., Stuttgart.

Pfaff, Dieter (1994): Zur Notwendigkeit einer eigenständigen Kostenrechnung Anmerkungen zur Neuorientierung des internen Rechnungswesens im Hause Siemens, in: Zeitschrift für betriebswirtschaftliche Forschung, 46. Jg., S. 1065-1084.

Pfaff, Dieter/Weber, Jürgen (1998): Zweck der Kostenrechnung? - Neue Sicht auf ein altes Problem, in: Die Betriebswirtschaft, 58. Jg., S. 151-165.

Pflesser, Christian (1999): Marktorientierte Unternehmenskultur - Konzeption und Untersuchung eines Mehrebenenmodells, Wiesbaden.

Pierce, Bernard/O'Dea, Tony (2003): Management Accounting Information and the Need of Managers - Perceptions of Managers and Accountants Compared, in: The British Accounting Review, Vol. 35, S. 257-290.

Podsakoff, Philip M./MacKenzie, Scott B./Lee, Jeong-Yeon/Podsakoff, Nathan P. (2003): Common Method Biases in Behavioral Research: A Critical Review of the Literature and Recommended Remedies, in: Journal of Applied Psychology, Vol. 88, S. 879-903.

Podsakoff, Philip M./Organ, Dennis W. (1986): Self-reports in Organizational Research: Problems and Prospects, in: Journal of Management, Vol. 12, S. 531544.

Preißler, Peter R. (2007): Controlling - Lehrbuch und Intensivkurs, 13. Aufl., München/Wien.

Reinecke, Jost (2005): Strukturgleichungsmodelle in den Sozialwissenschaften, München.

Sandt, Joachim (2003): Kennzahlen für die Unternehmensführung - verlorenes Heimspiel für Controller?, in: Zeitschrift für Controlling \& Management, 47. Jg., S. 75-79. 
Scapens, Robert W./Jazayeri, Mostafa (2003): ERP Systems and Management Accounting Change: Opportunities or Impacts? - A Research Note, in: European Accounting Review, Vol. 12, S. 201-233.

Schäffer, Utz/Zyder, Michael (2007): Eine Analyse des moderierenden Einflusses der Faktoren Wettbewerbsintensität, Marktdynamik und dezentrale Autonomie auf die erfolgreiche Gestaltung der Budgetierung, in: Zeitschrift für Planung \& Unternehmenssteuerung, $18 \mathrm{Jg}$., S. 7-33.

Schaier, Sven (2007): Konvergenz von internem und externem Rechnungswesen Bedarf für eine Neustrukturierung des Rechnungswesens?, Wiesbaden.

Schermelleh-Engel, Karin/Moosbrugger, Helfried/Müller, Hans (2003): Evaluating the Fit of Structural Equation Models: Tests of Signifiance and Descriptive Goodness-of-Fit Measures, in: Methods of Psychological Research, Vol. 8, S. 2374.

Schildbach, Thomas (1997): Cost Accounting in Germany, in: Management Accounting Research, Vol. 8, S. 261-276.

Schmalenbach, Eugen (1962): Dynamische Bilanz, 13. Aufl., Köln/Opladen.

Schmitz, Frank (2006): Optimierung und Harmonisierung des internen und externen Berichtswesens im IFRS-Umfeld, in: Accounting, 6. Jg., Heft 8, S. 11-15.

Schneider, Dieter (1997): Betriebswirtschaftslehre - Band 2: Rechnungswesen, 2. Aufl., München/Wien.

Schnell, Rainer/Hill, Paul B./Esser, Elke (2008): Methoden der empirischen Sozialforschung, 8. Aufl., München/Wien.

Scholderer, Joachim/Balderjahn, Ingo (2006): Was unterscheidet harte und weiche Strukturgleichungsmodelle nun wirklich? - Ein Klärungsversuch zur LISRELPLS-Frage, in: Marketing: Zeitschrift für Forschung und Praxis, 28. Jg., S. 57-70.

Scholderer, Joachim/Balderjahn, Ingo/Paulssen, Marcel (2006): Kausalität, Linearität, Reliabilität: Drei Dinge, die Sie nie über Strukturgleichungsmodelle wissen wollten, in: Die Betriebswirtschaft, 66. Jg., S. 640-650. 
Schruff, Wienand/Melcher, Winfried (Hrsg.) (2009): Umsetzung der HGBModernisierung - Einführung, Überblick und ausgewählte kritische Diskussionsbeiträge zum Bilanzrechtsmodernisierungsgesetz (BilMoG), Beilage 5/2009 zu Heft 23, Der Betrieb, 62. Jg.

Schweitzer, Marcell (2003): Leitungsebenendifferenzierte Kennzahlen als Instrumente des Controllings, in: Jahnke, Hermann/Brüggemann, Wolfgang (Hrsg.): Betriebswirtschaftslehre und betriebliche Praxis - Festschrift für Horst Seelbach zum 65. Geburtstag, Wiesbaden, S. 429-454.

Sharma, Subhash/Durand, Richard M./Gur-Arie, Oded (1981): Identification and Analysis of Moderator Variables, in: Journal of Marketing Research, Vol. 18, S. 291-300.

Shrout, Patrick E./Bolger, Niall (2002): Mediation in Experimental and Nonexperimental Studies: New Procedures and Recommendations, in: Psychological Methods, Vol. 7, S. 422-445.

Simons, Dirk/Weißenberger, Barbara E. (2008): Die Konvergenz von externem und internem Rechnungswesen - Kritische Faktoren für die Entwicklung einer partiell integrierten Rechnungslegung aus theoretischer Sicht, in: Betriebswirtschaftliche Forschung und Praxis, 60. Jg., S. 137-160.

Simons, Dirk/Weißenberger, Barbara E. (2009a): „Different costs for different purposes" vs. „one version of the trut"? - Zur Konvergenz von externer und interner Rechnungslegung im deutschsprachigen Raum (Teil 1), in: Wirtschaftswissenschaftliches Studium, 38. Jg., S. 390-395.

Simons, Dirk/Weißenberger, Barbara E. (2009b): „Different costs for different purposes” vs. „one version of the truth“? - Zur Konvergenz von externer und interner Rechnungslegung im deutschsprachigen Raum (Teil 2), in: Wirtschaftswissenschaftliches Studium, 38. Jg., S. 446-451.

Smith, David/Langfield-Smith, Kim (2004): Structural Equation Modeling in Management Accounting Research: Critical Analysis and Opportunities, in: Journal of Accounting Literature, Vol. 23, S. 49-86. 
Söhnchen, Florian (2007): Common Method Variance und Single Source Bias, in: Albers, Sönke/Klapper, Daniel/Konradt, Udo/Walter, Achim/Wolf, Joachim (Hrsg.): Methodik der empirischen Forschung, 2. Aufl., Wiesbaden, S. 135-150.

Sorter, George H./Becker, Selwyn W./Archibald, T. R./Beaver, W. (1964): Corporate Personality as Reflected in Accounting Decisions: Some Preliminary Findings, in: Journal of Accounting Research, Vol. 2, S. 183-196.

Spillecke, Dennis (2006): Interne Kundenorientierung des Controllerbereichs - Messung - Erfolgsauswirkungen - Determinanten, Wiesbaden.

Steiger, James H. (1990): Structural Model Evaluation and Modification: An Interval Estimation Approach, in: Multivariate Behavioral Research, Vol. 25, S. 173-180.

Stewart, G. Bennett (1991): The Quest for Value, New York.

Stewart, G. Bennett (1994): EVA: Fact and Fantasy, in: Journal of Applied Corporate Finance, Vol. 7, S. 71-84.

Stone, Eugene F. (1988): Moderator Variables in Research: A Review and Analysis of Conceptual and Methodological Issues, in: Research in Personnel and Human Resources Management, Vol. 6, S. 191-229.

Sudman, Seymour/Bradburn, Norman M. (1982): Asking Questions - A Practical Guide to Questionnaire Design, San Francisco.

Temme, Dirk/Paulssen, Marcel/Hildebrandt, Lutz (2009): Common Method Variance - Ursachen, Auswirkungen und Kontrollmöglichkeiten, in: Die Betriebswirtschaft, 69. Jg., S. 123-146.

Tucker, Ledyard R./Lewis, Charles (1973): A Reliability Coefficient for Maximum Likelihood Factor Analysis, in: Psychometrika, Vol. 38, S. 1-10.

Van der Stede, Wim A./Young, S. Mark/Chen, Clara Xiaoling (2005): Assessing the Quality of Evidence in Empirical Management Accounting Research: The Case of Survey Studies, in: Accounting, Organizations and Society, Vol. 30, S. 655-684. 
Velte, Patrick (2008): ZP-Stichwort: Management Approach, in: Zeitschrift für Planung \& Unternehmenssteuerung, 19. Jg., S. 133-138.

Wagenhofer, Alfred (2006): Zusammenwirken von Controlling und Rechnungslegung nach IFRS, in: Wagenhofer, Alfred (Hrsg.): Controlling und IFRSRechnungslegung - Konzepte, Schnittstellen, Umsetzung, Berlin, S. 1-20.

Wagenhofer, Alfred (2008): Konvergenz von intern und extern berichteten Ergebnisgrößen am Beispiel von Segmentergebnissen, in: Betriebswirtschaftliche Forschung und Praxis, 60. Jg., S. 161-176.

Wagenhofer, Alfred/Engelbrechtsmüller, Christian (Hrsg.) (2006): Controlling und Reporting vor dem Hintergrund der Anforderungen von Internationalen Rechnungslegungsstandards, Graz/Linz.

Währisch, Michael (1998): Kostenrechnungspraxis in der deutschen Industrie - Eine empirische Studie, Wiesbaden.

Wala, Thomas/Knoll, Leonhard/Messner, Stephanie (2007): Vor- und Nachteile einer Integration von internem und externem Rechnungswesen auf Basis der IFRS - Teil II, in: Deutsches Steuerrecht, 45. Jg., S. 1881-1883.

Watrin, Christoph/Pott, Christiane (2008): Einfachheit versus Komplexität von Rechnungslegungsinformationen: Empirische Befunde, in: vom Brocke, Jan/Becker, Jörg (Hrsg.): Einfachheit in Wirtschaftsinformatik und Controlling, S. 437-448.

Weaver, Samuel C. (2001): Measuring Economic Value Added: A Survey of the Practices of EVA Proponents, in: Journal of Applied Finance, Vol. 11, S. 50-60.

Weber, Jürgen (2002): Betriebswirtschaftliche Instrumente - Segen oder Fluch?, in: Kostenrechnungspraxis, 46. Jg., S. 339-340.

Weber, Jürgen (2008): Von Top-Controllern lernen - Controlling in den DAX 30Unternehmen, Weinheim.

Weber, Jürgen (2009): Erfahrungen mit wertorientierter Steuerung, in: Der Betrieb, 62. Jg., S. 297-303. 
Weber, Jürgen/Grothe, Martin/Schäffer, Utz (2000): ZP-Stichwort: Mentale Modelle, in: Zeitschrift für Planung, 11. Jg., S. 239-244.

Weber, Jürgen/Hirsch, Bernhard/Rambusch, René/Schlüter, Hendrik/Sill, Frauke/Spatz, Almuth (2006): Controlling 2006 - Stand und Perspektiven, Vallendar.

Weber, Jürgen/Schäffer, Utz (2008): Einführung in das Controlling, 12. Aufl., Stuttgart.

Weber, Jürgen/Vater, Hendrik/Schmidt, Walter/Reinhard, Hartmut/Ernst, Edgar (Hrsg.) (2008): Die neue Rolle des Controllers - Aufgaben, Anforderungen, Best Practices, Stuttgart.

Weber, Jürgen/Weißenberger, Barbara E. (2001): Finanzorientierung - die neue Herausforderung für das Controlling im internationalen Unternehmen, in: Krystek, Ulrich/Zur, Eberhard (Hrsg.): Handbuch Internationalisierung, 2. Aufl., Berlin et al., S. 541-569.

Weber, Jürgen/WeiBenberger, Barbara E. (2006): Einführung in das Rechnungswesen, 7. Aufl., Stuttgart.

Weber, Jürgen/Willauer, Bianca/Schäffer, Utz (2003): Einführung zur Skalenübersicht, in: Weber, Jürgen/Kunz, Jennifer (Hrsg.): Empirische Controllingforschung - Begründung - Beispiele - Ergebnisse, Wiesbaden, S. 369-383.

Weber, Wolfgang/Kabst, Rüdiger (2009): Einführung in die Betriebswirtschaftslehre, 7. Aufl., Wiesbaden.

Weide, Gonn (2009): Gestaltung und Erfolg des Management Reporting - Empirische Analyse der Auswirkungen einer Integration des Rechnungswesens, Hamburg.

Weißenberger, Barbara E. (1997): Die Informationsbeziehung zwischen Management und Rechnungswesen - Analyse institutionaler Koordination, Wiesbaden.

Weißenberger, Barbara E. (2002): Controlling als Teilgebiet der Betriebswirtschaftslehre - konzeptionelle Einordnung und Konsequenzen für Forschung und Lehre, in: Weber, Jürgen/Hirsch, Bernhard (Hrsg.): Controlling als akademische Disziplin. Eine Bestandsaufnahme, Wiesbaden, S. 389-407. 
Weißenberger, Barbara E. (2003): Anreizkompatible Erfolgsrechnung im Konzern Grundmuster und Gestaltungsalternativen, Wiesbaden.

Weißenberger, Barbara E. (2004): Theoretische Grundlagen der Erfolgsmessung im Controlling, in: Scherm, Ewald/Pietsch, Gotthard (Hrsg.): Controlling - Theorie und Konzeptionen, München, S. 289-313.

Weißenberger, Barbara E. (2005): Controlling unter IFRS - Möglichkeiten und Grenzen einer integrierten Erfolgsrechnung, in: Weber, Jürgen/Meyer, Matthias (Hrsg.): Internationalisierung des Controllings, Wiesbaden, S. 185-212.

Weißenberger, Barbara E. (2006a): Controller und IFRS: Konsequenzen der IFRSFinanzberichterstattung für die Controlleraufgaben, in: Zeitschrift für internationale und kapitalmarktorientierte Rechnungslegung, 6. Jg., S. 613-622.

Weißenberger, Barbara E. (2006b): Controller unter IFRS: Konsequenzen einer IFRS-Finanzberichterstattung für die Aufgabenfelder von Controllern, in: Betriebswirtschaftliche Forschung und Praxis, 58. Jg., S. 342-364.

Weißenberger, Barbara E. (2007a): IFRS für Controller - Einführung, Anwendung, Fallbeispiele, Freiburg et al.

Weißenberger, Barbara E. (2007b): Auswirkungen der IFRS auf das Controlling, in: Freidank, Carl-Christian/Tanski, Joachim (Hrsg.): Management-Handbuch Accounting, Controlling \& Finance, 5. Aufl., München, S. 1-29.

Weißenberger, Barbara E. (2007c): Zum grundsätzlichen Verhältnis von Controlling und externer Finanzberichterstattung unter IFRS, in: Der Konzern, 5. Jg., S. 321331.

Weißenberger, Barbara E. (2008a): $§ 52$ IFRS-Rechnungslegung und Controlling, in: Lüdenbach, Norbert/Hoffmann, Wolf-Dieter (Hrsg.): IFRS Kommentar, 6. Aufl., Freiburg et al., S. 2461-2498.

Weißenberger, Barbara E. (2008b): Controller und IFRS - Konsequenzen der IFRSFinanzberichterstattung für die Controlleraufgaben, in: Funk, Wilfried/Rossmanith, Jonas (Hrsg.): Internationale Rechnungslegung und Internationales Controlling, Wiesbaden, S. 425-454. 
Weißenberger, Barbara E./Angelkort, Hendrik (2007a): IFRS-Rechnungslegung und Controlling, in: Heyd, Reinhard/von Keitz, Isabel (Hrsg.): IFRS-Management, München, S. 409-437.

Weißenberger, Barbara E./Angelkort, Hendrik (2007b): Controller Excellence unter IFRS in Österreich - Eine Studie der Justus-Liebig-Universität Gießen mit Unterstützung des Österreichischen Controller-Instituts, Wien.

Weißenberger, Barbara E./Haas, Cornelia A. J./Wolf, Sebastian (2007): GoodwillControlling unter IAS 36 - Konzeptionelle Überlegungen und Gestaltungsvorschläge, in: Praxis der internationalen Rechnungslegung, 3. Jg., S. 149-156.

Weißenberger, Barbara E./Maier, Michael (2006): Der Management Approach in der IFRS-Rechnungslegung: Fundierung der Finanzberichterstattung durch Informationen aus dem Controlling, in: Der Betrieb, 59. Jg., S. 2077-2083.

Wenzel, Christian (2007): Neuausrichtung der Konzernsteuerung und Harmonisierung der internen und externen Berichterstattung in einem multi-divisionalen Konzern, in: Horváth, Péter (Hrsg.): Erfolgstreiber für das Controlling, Stuttgart, S. 299-308.

West, Stephen G./Finch, John F./Curran, Patrick J. (1995): Structural Equation Models with Nonnormal Variables: Problems and Remedies, in: Hoyle, Rick. H. (Hrsg.): Structural Equation Modeling - Concepts, Issues, and Applications, Thousand Oaks et al., S. 56-75.

Wood, Robert E./Goodman, Jodi S./Beckmann, Nadin/Cook, Alison (2008): Mediation Testing in Management Research - A Review and Proposals, in: Organizational Research Methods, Vol. 11, S. 270-295.

Ziegler, Hasso (1994): Neuorientierung des internen Rechnungswesens für das Unternehmens-Controlling im Hause Siemens, in: Zeitschrift für betriebswirtschaftliche Forschung, 46. Jg., S. 175-188.

Zimmerman, Jerold L. (2001): Conjectures Regarding Empirical Managerial Accounting Research, in: Journal of Accounting \& Economics, Vol. 32, S. 411-427. 
Zinnbauer, Markus/Eberl, Markus (2004): Die Überprüfung von Spezifikation und Güte von Strukturgleichungsmodellen: Verfahren und Anwendung, Schriften zur Empirischen Forschung und Quantitativen Unternehmensplanung, Heft 21/2004, München.

Zoni, Laura/Merchant, Kenneth A. (2007): Controller Involvement in Management: An Empirical Study in Large Italian Corporations, in: Journal of Accounting \& Organizational Change, Vol. 3, S. 29-43. 


\section{Controlling \& Business Accounting}

Herausgegeben von Prof. Dr. Barbara E. Weißenberger

Band 1 Michael Maier: Der Management Approach. Herausforderungen für Controller und Abschlußprüfer im Kontext der IFRS-Finanzberichterstattung. 2009.

Band 2 Christoph Gehrig: Anwendungssystemgestütztes strategisches Controlling. Konzeption und empirische Erkenntnisse. 2009.

Band 3 Hendrik Angelkort: Integration des Rechnungswesens als Erfolgsfaktor für die Controllerarbeit. Eine empirische Untersuchung deutscher Großunternehmen. 2010.

www.peterlang.de 


\section{Strategische Planung, Steuerung und Kontrolle von Mergers \& Acquisitions} M\&A-Controlling - M\&A-Risikomanagement - Praxiswissen

Frankfurt am Main, Berlin, Bern, Bruxelles, New York, Oxford, Wien, 2009. XVII, 324 S., 103 Abb.

Controlling und Management. Herausgegeben von Thomas Reichmann und Martin K. Welge. Bd. 39 ISBN 978-3-631-59726-2 · geb. €59,80*

Für zahlreiche Branchen ist angesichts der hohen Branchendynamik eine Wachstumsstrategie von essentieller Bedeutung, um den Erwartungen der Shareholder zu entsprechen. Da internes Wachstum häufig nicht schnell genug generiert werden kann, ist externes Wachstum durch M\&A eine häufig gewählte Option. Empirische Studien belegen allerdings, dass in der Praxis eine Vielzahl von M\&A-Transaktionen auf Grund unzureichender strategischer Planung, Steuerung und Kontrolle scheitert. Zielsetzung der Arbeit ist es daher, Empfehlungen zu formulieren, mit deren Hilfe die strategische Unternehmensentwicklung durch Mergers \& Acquisitions erfolgreicher gestaltet werden kann. Des Weiteren wird ein Modell zur prozessorientierten M\&A-Strategieentwicklung entworfen, das die prozessbegleitende strategische Planung, Steuerung und Kontrolle von M\&A-Transaktionen gewährleistet und dadurch systematisch die strategischen Implikationen eines Deals identifiziert. Das in diesem Zusammenhang entwickelte und vorgestellte strategische Controlling von M\&A ist allgemein als systematische Entscheidungsunterstützung für die praktische Anwendung in Unternehmen zu verstehen.

Aus dem Inhalt: Ausführliche Definition und Theorieherleitung · Prozessmodell der M\&A-Strategieentwicklung · Bereitstellung eines nutzbaren Instrumentariums zur holistischen Bewertung einer M\&A-Strategie · Risikomanagement als wichtiger Bestandteil von M\&A

Frankfurt am Main - Berlin - Bern - Bruxelles - New York · Oxford · Wien

Auslieferung: Verlag Peter Lang AG

Moosstr. 1, $\mathrm{CH}-2542$ Pieterlen

Telefax 0041 (0) 32/3761727

*inklusive der in Deutschland gültigen Mehrwertsteuer

Preisänderungen vorbehalten 
Hendrik Angelkort - 978-3-631-75143-5

Downloaded from PubFactory at 01/11/2019 07:47:42AM

via free access 
Hendrik Angelkort - 978-3-631-75143-5

Downloaded from PubFactory at 01/11/2019 07:47:42AM

via free access 